Supporting Information

for

\title{
Diastereoselective Protonation on Radical Anions of Electron- Deficient Alkenes via Photoinduced Electron Transfer
}

Tomoo Hayamizu, Hajime Maeda, and Kazuhiko Mizuno*

Department of Applied Chemistry, Graduate School of Engineering, Osaka Prefecture University, 1-1 Gakuen-cho, Sakai, Osaka 599-8531, Japan

mizuno@chem.osakafu-u.ac.jp

\section{Contents}

S2 - S9 $\quad{ }^{1} \mathrm{H}$ and ${ }^{13} \mathrm{C}$ NMR spectra of 1a-d.

S10 - S51 $\quad{ }^{1} \mathrm{H}$ and ${ }^{13} \mathrm{C}$ NMR spectra of 3a, 4a, trans-6a, trans-3b, trans-4b, trans- $6 \mathbf{b}, \mathbf{3 c}$, cis- $4 \mathbf{c}$, cis-6c, endo-3d, endo-4d, 6d, 3e, endo-4e, $6 \mathrm{e}$.

S52 - S70 X-ray crystallographic Data of cis-3a.

S71 - S92 X-ray crystallographic Data of trans-6a.

S93 - S111 X-ray crystallographic Data of trans-6b.

S112 - S129 X-ray crystallographic Data of cis-6c.

S130 - S146 X-ray crystallographic Data of endo-6d.

S147 - S169 X-ray crystallographic Data of endo-6e.

S170 Summary of Crystallographic Data

S171 General Experimental Method 


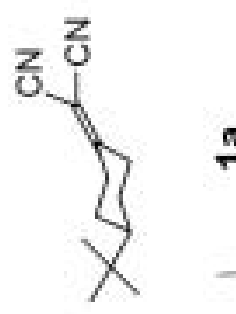

$\stackrel{2}{-}$

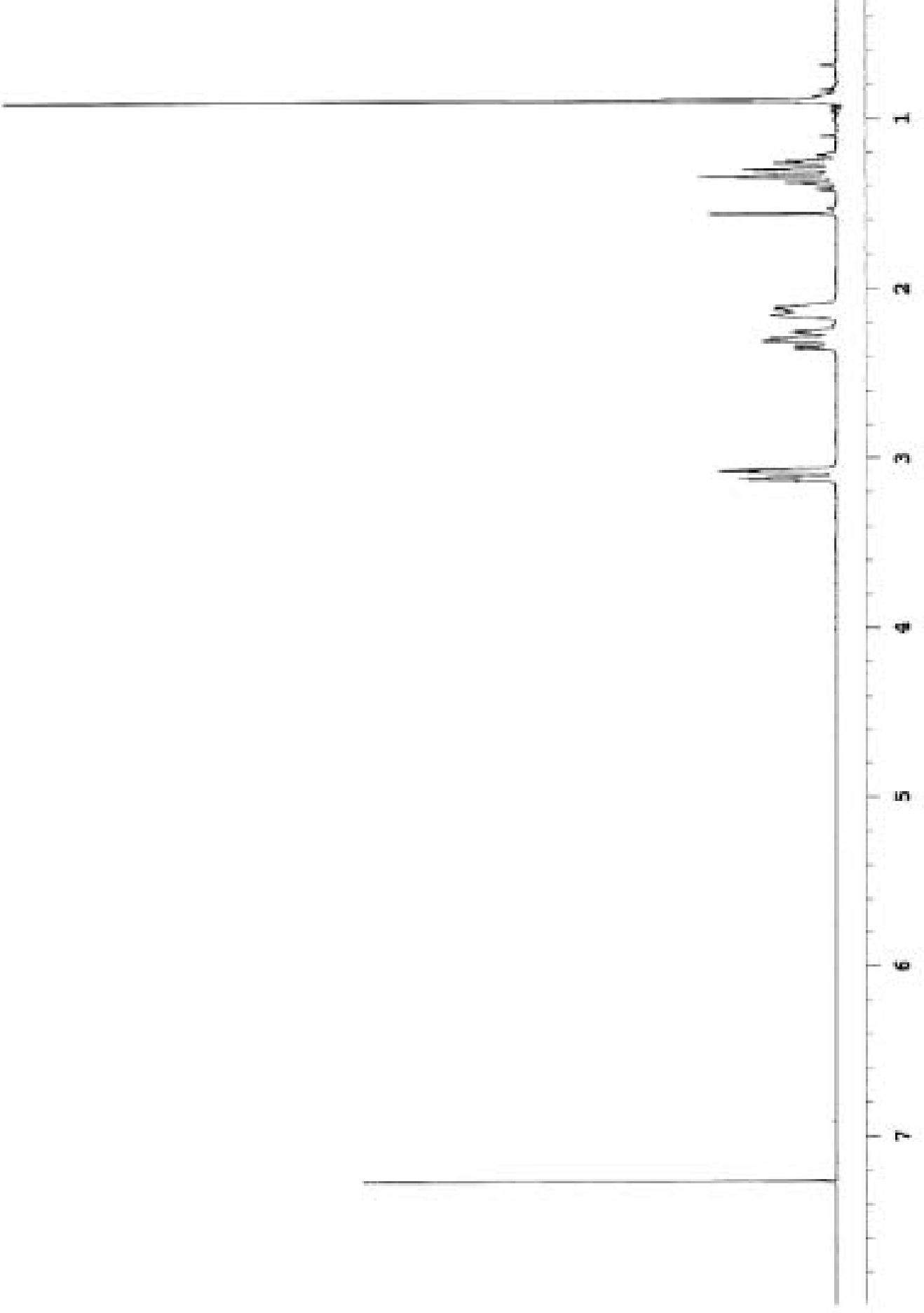



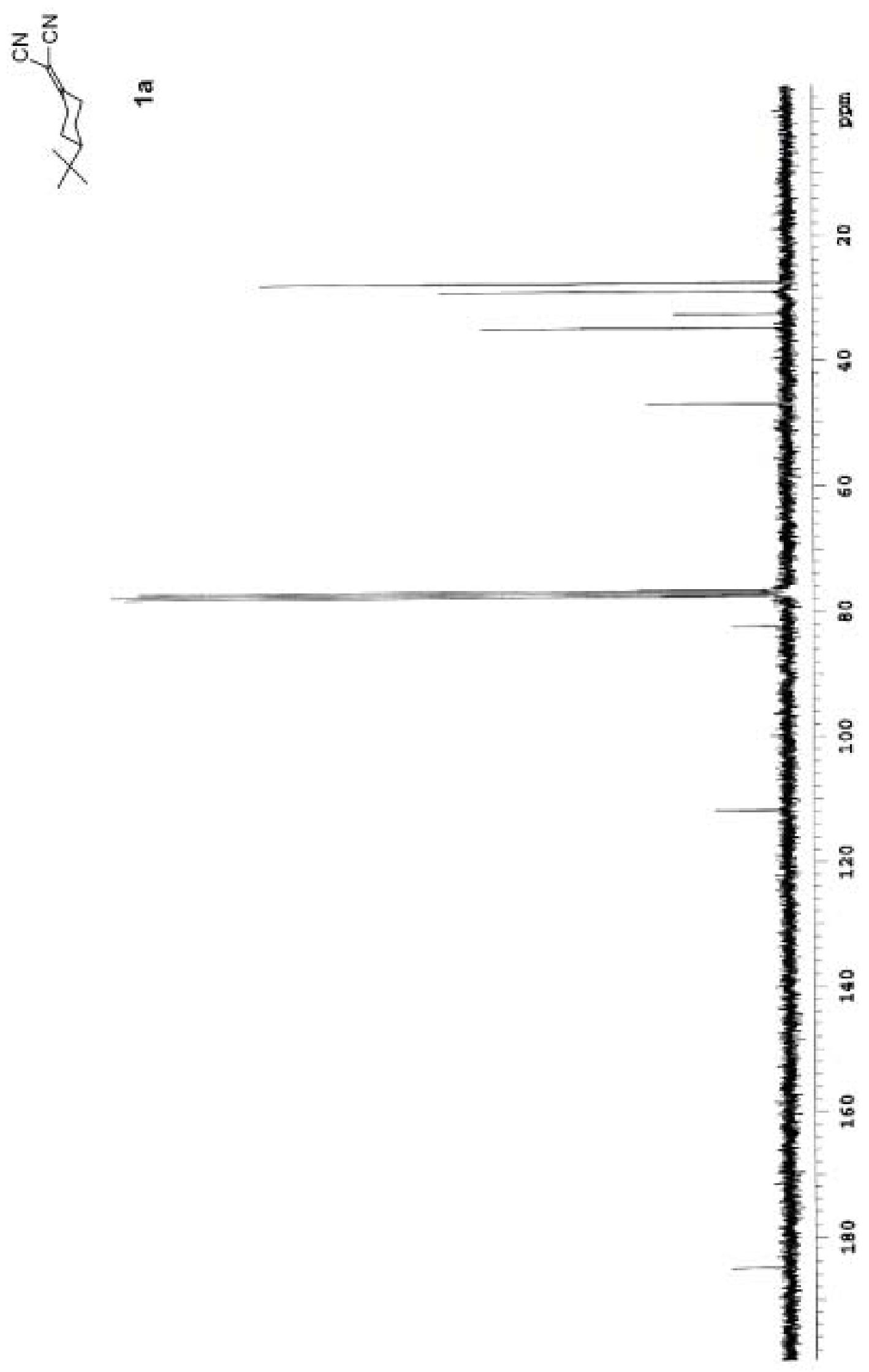

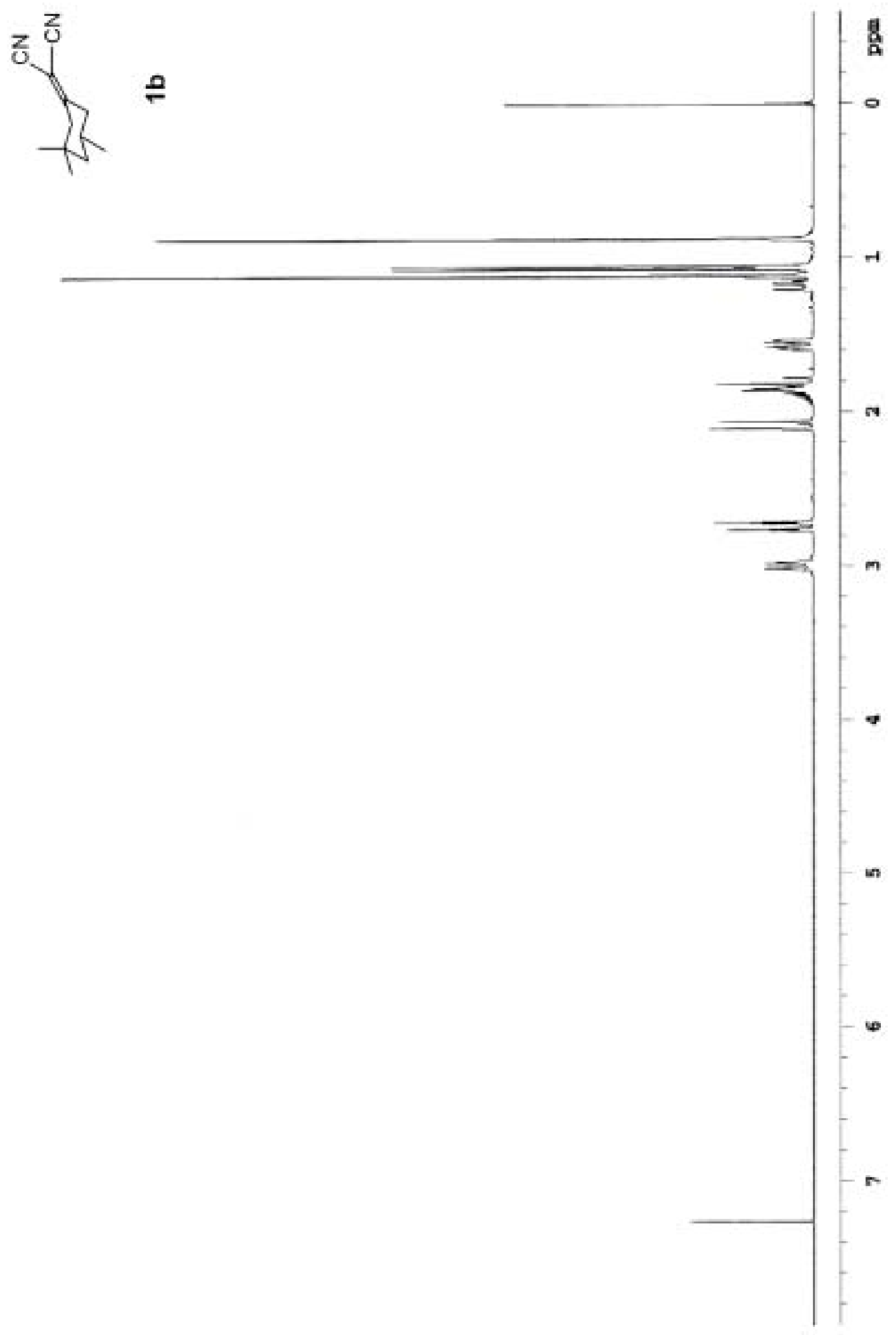

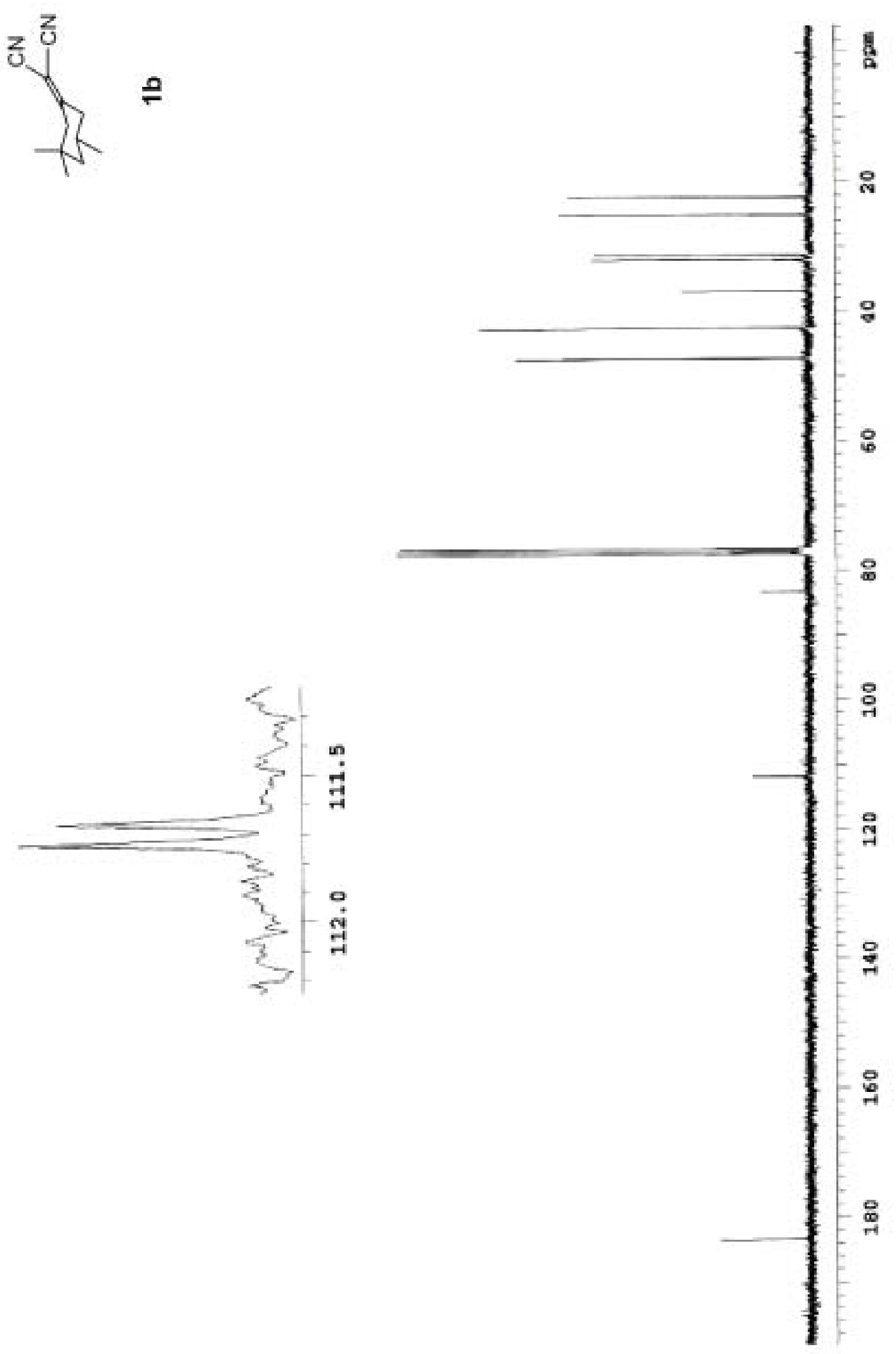

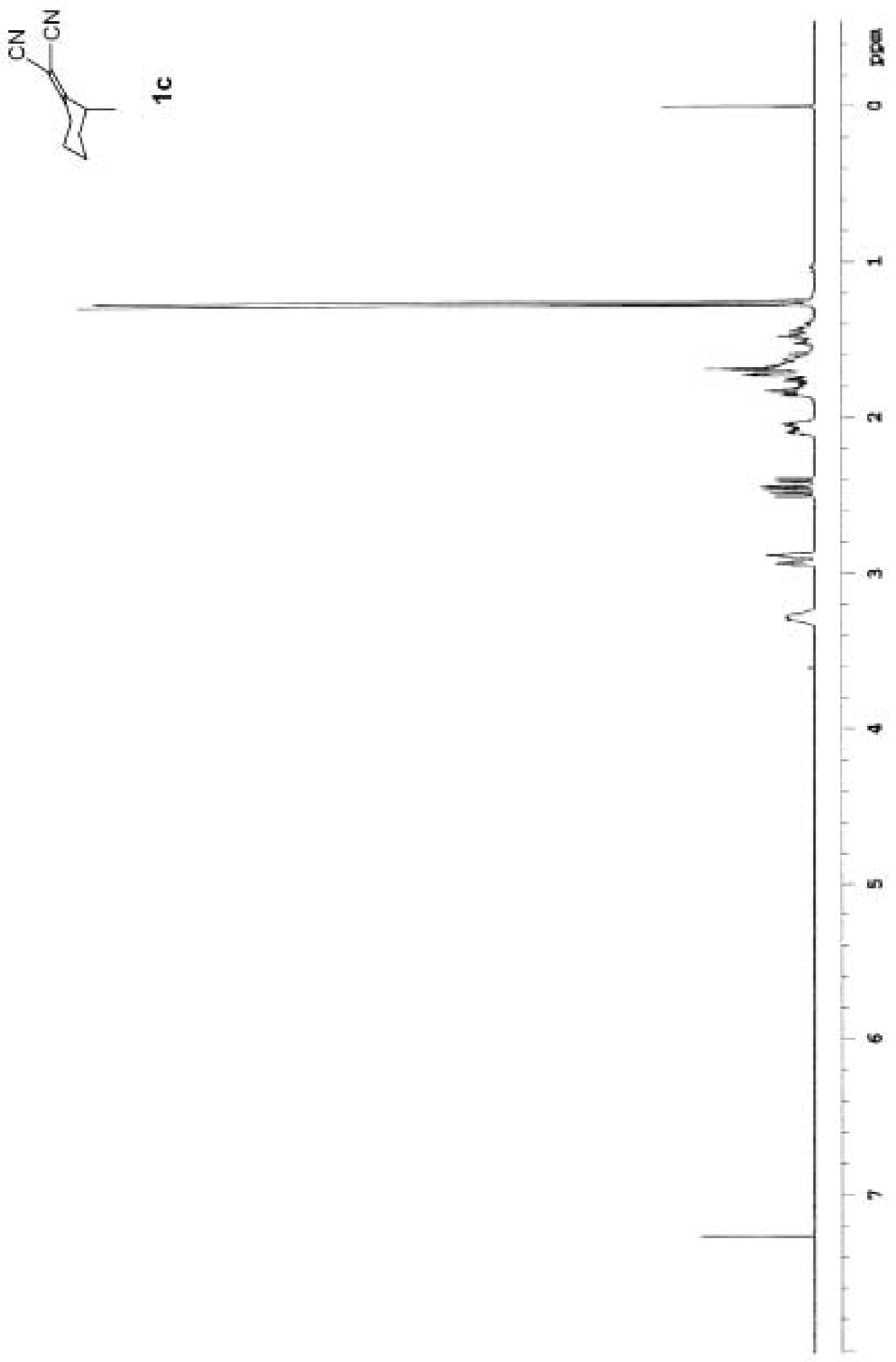

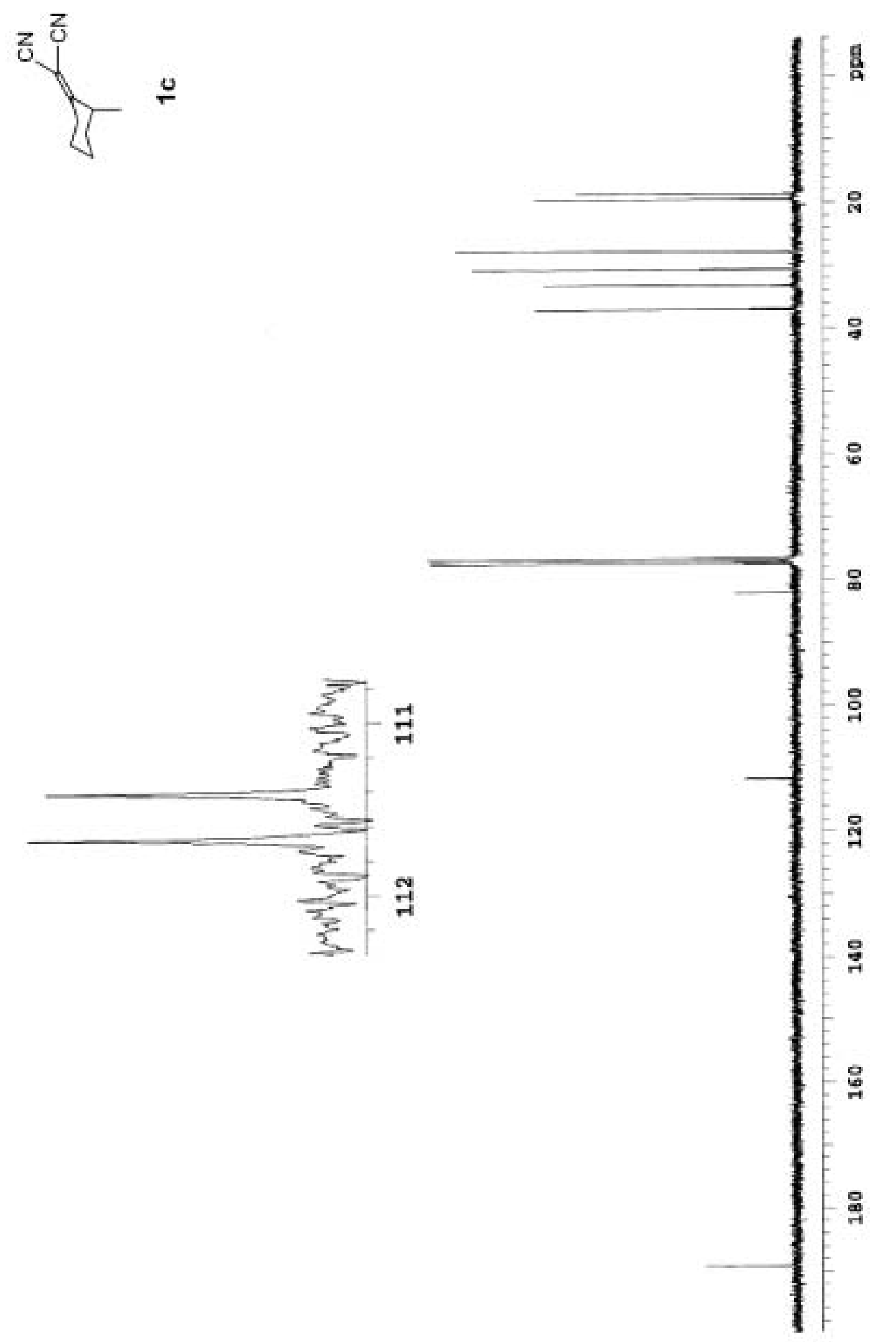

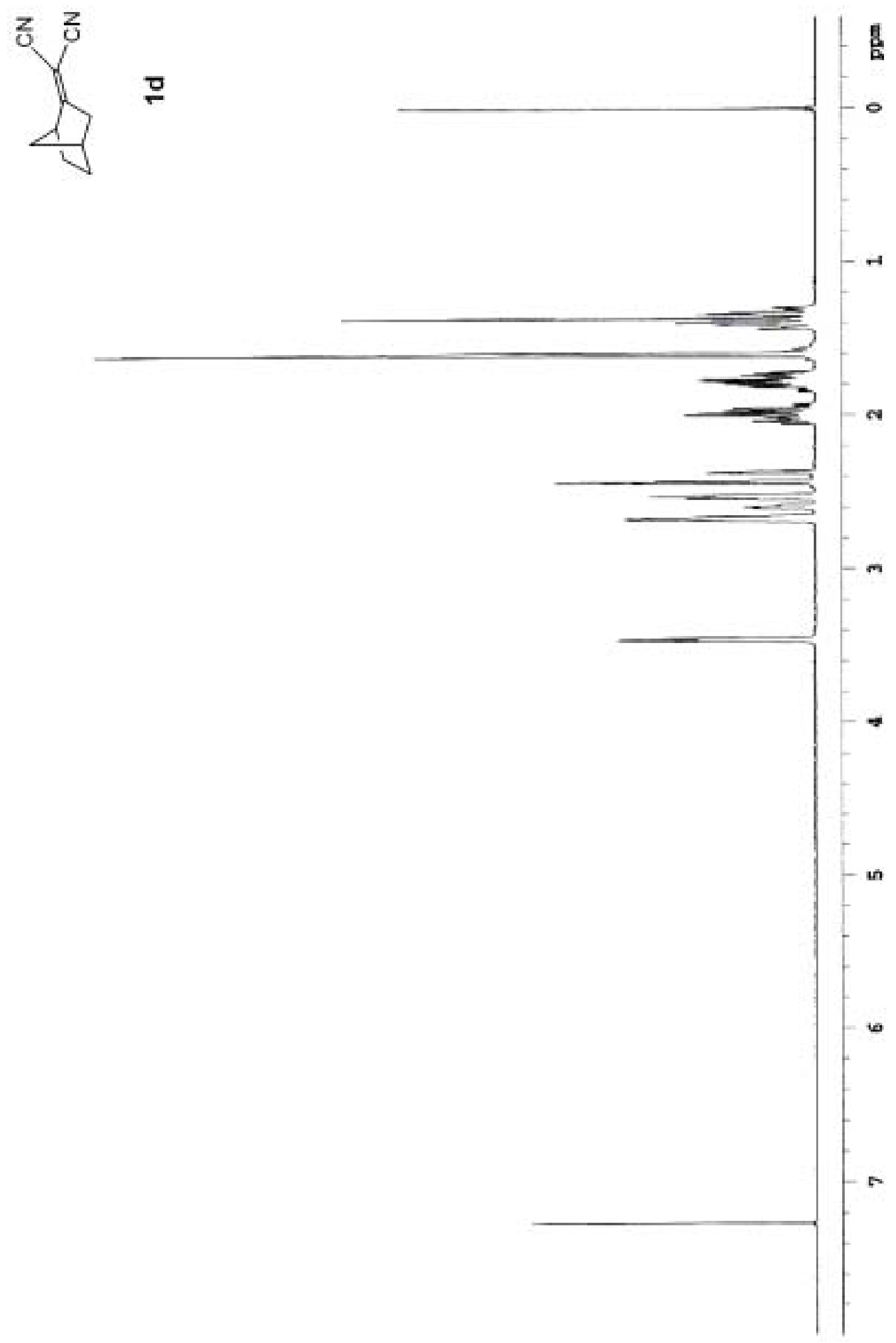

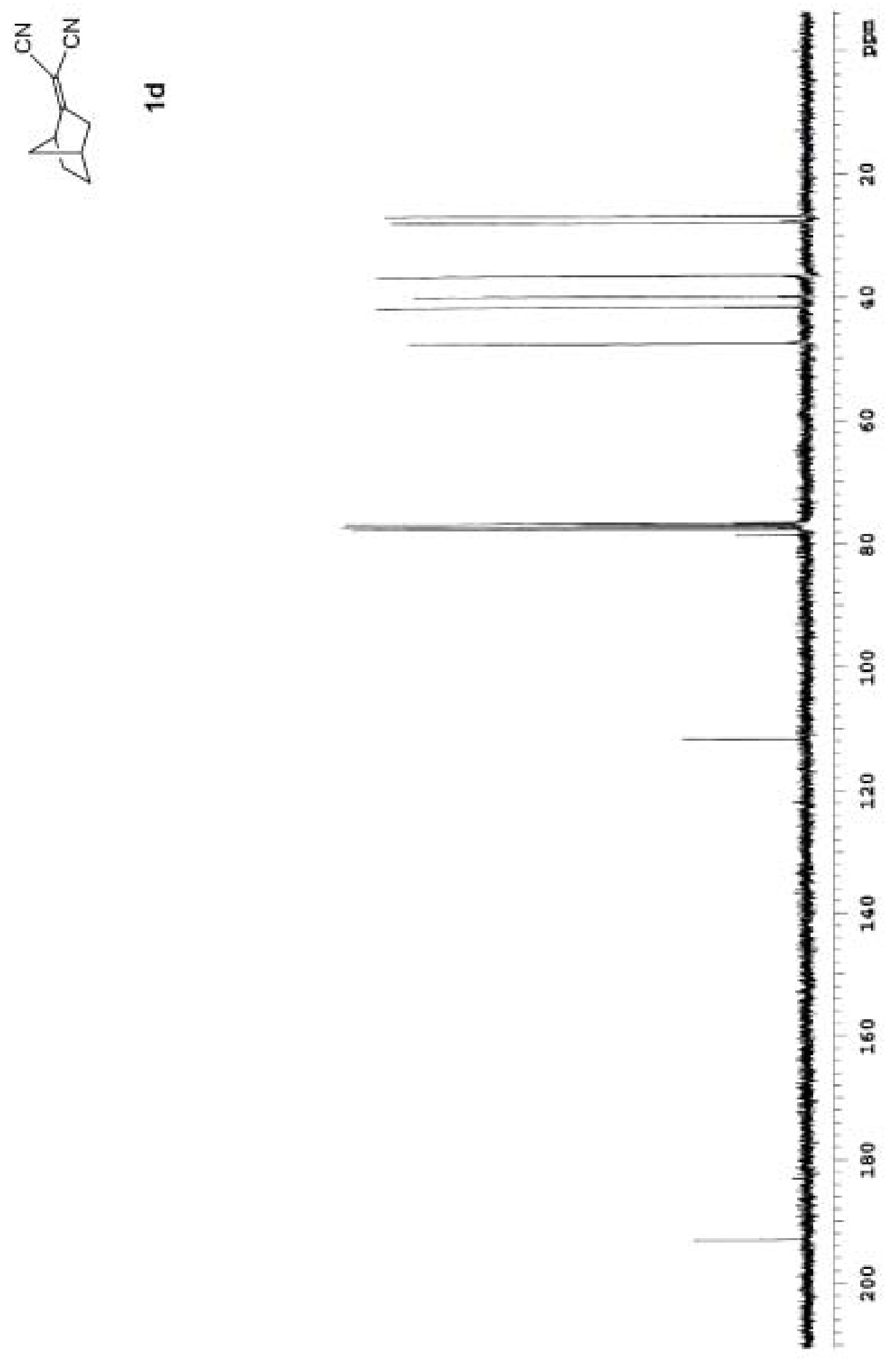


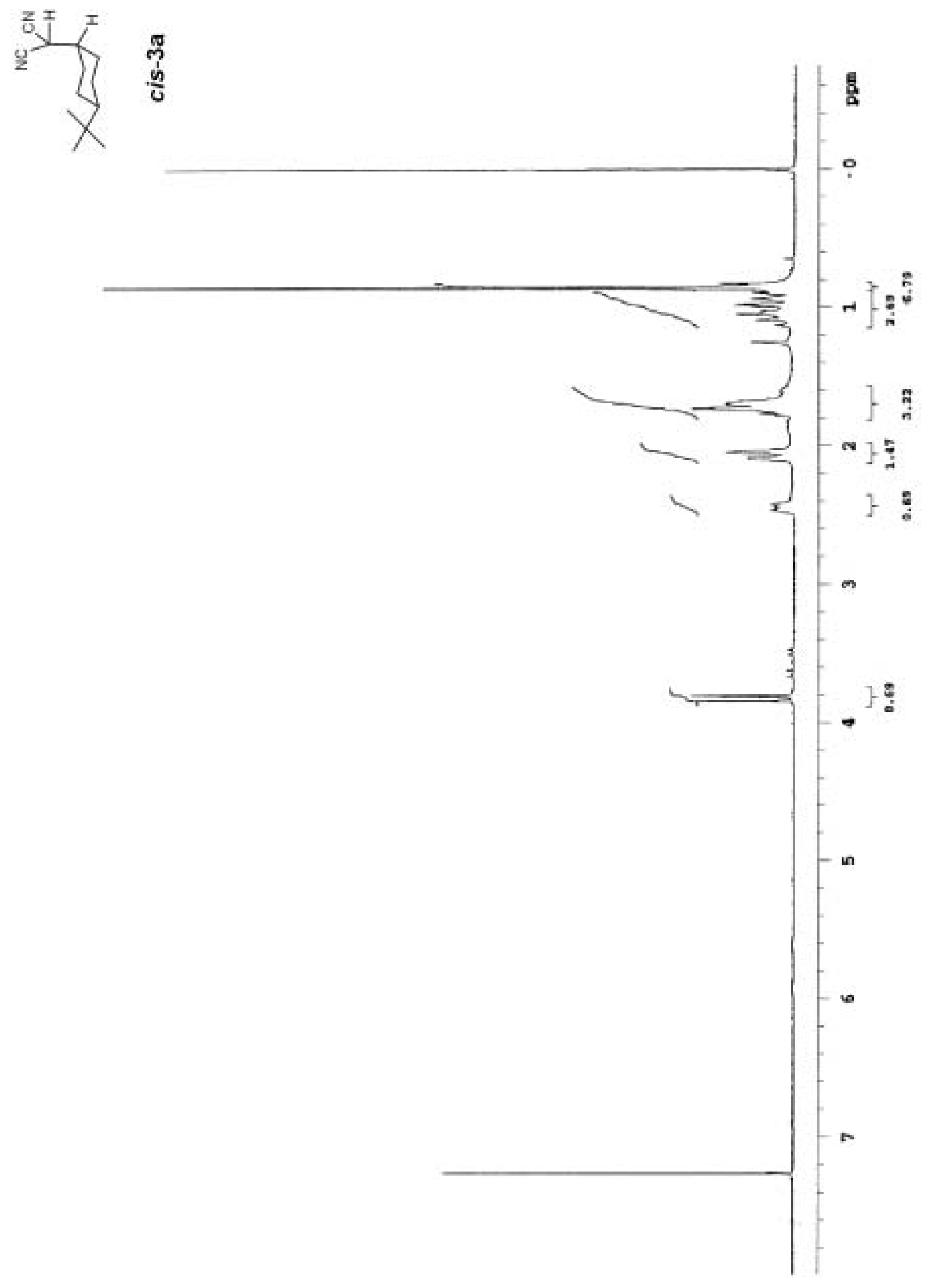


$\underbrace{I}_{X}$

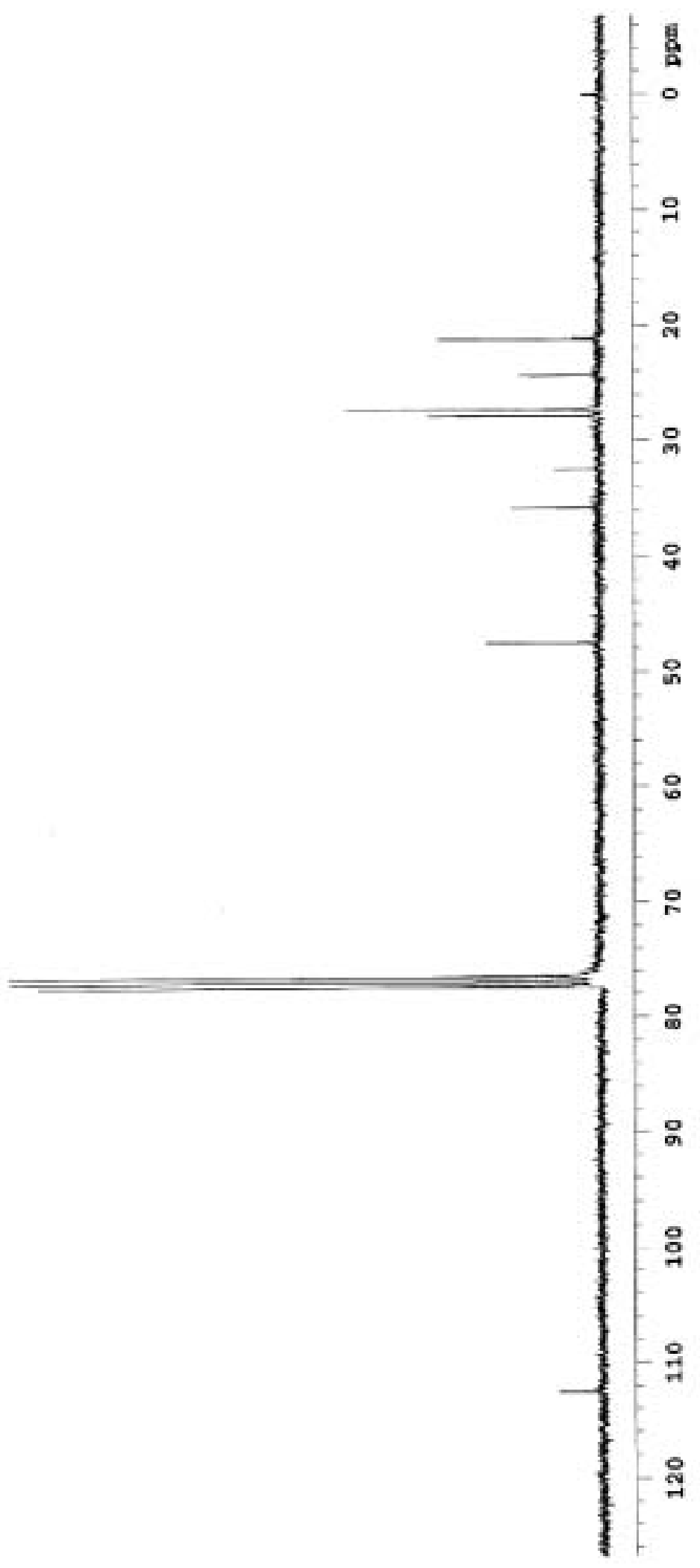



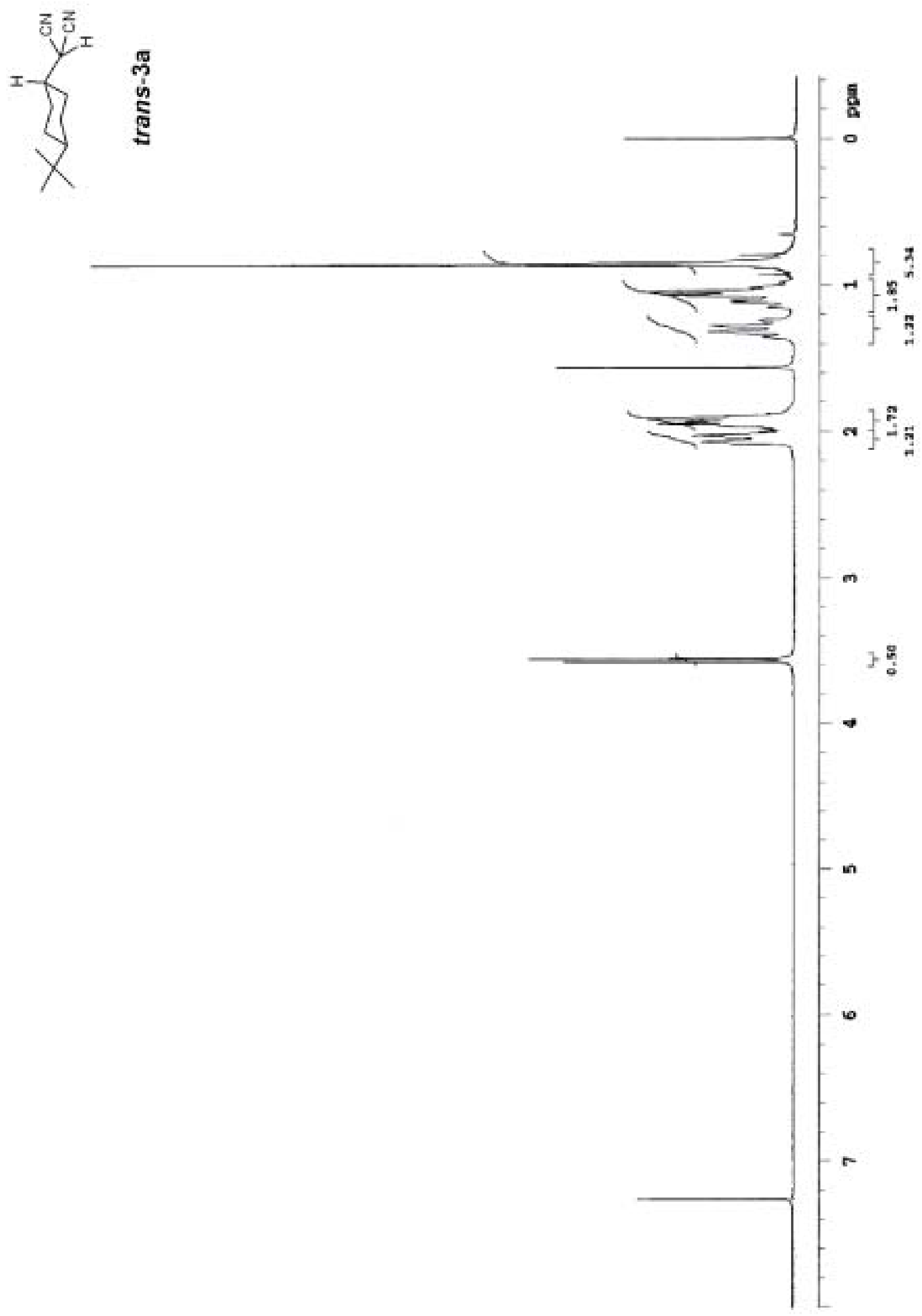

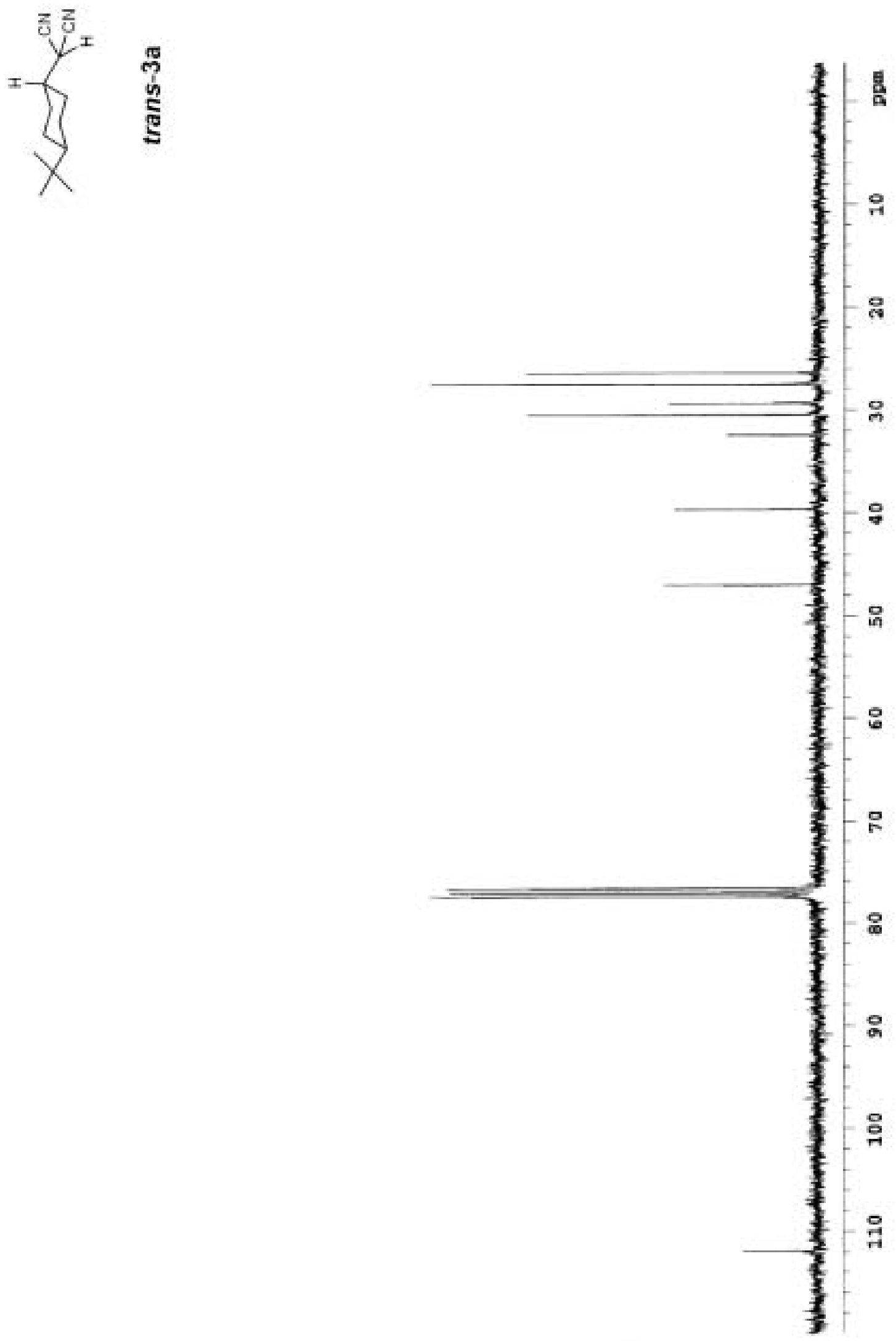
$\sum^{2} \frac{3}{4}$

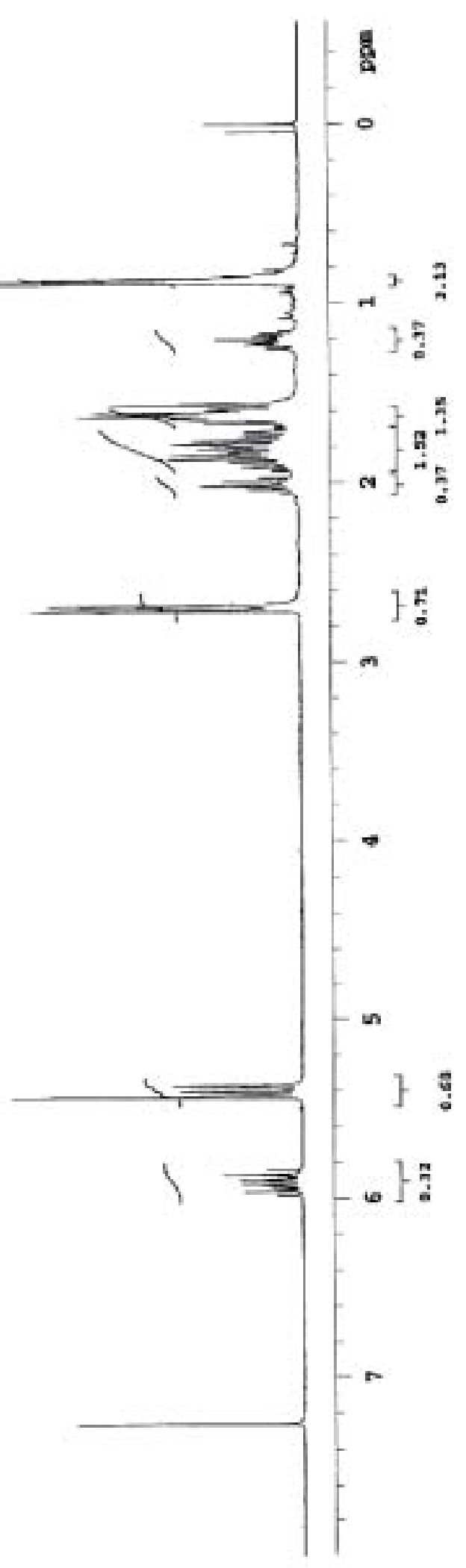


$\sum_{2}^{2} \frac{2}{4}$

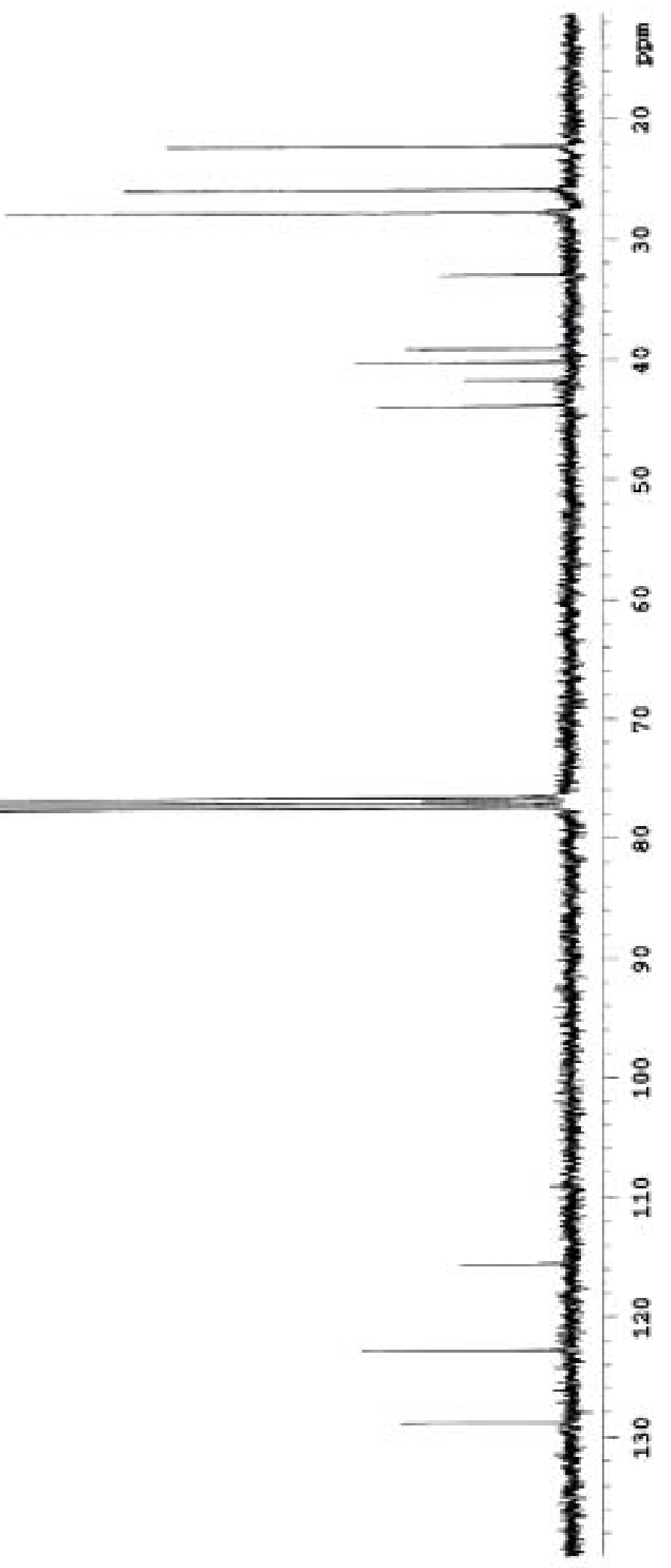



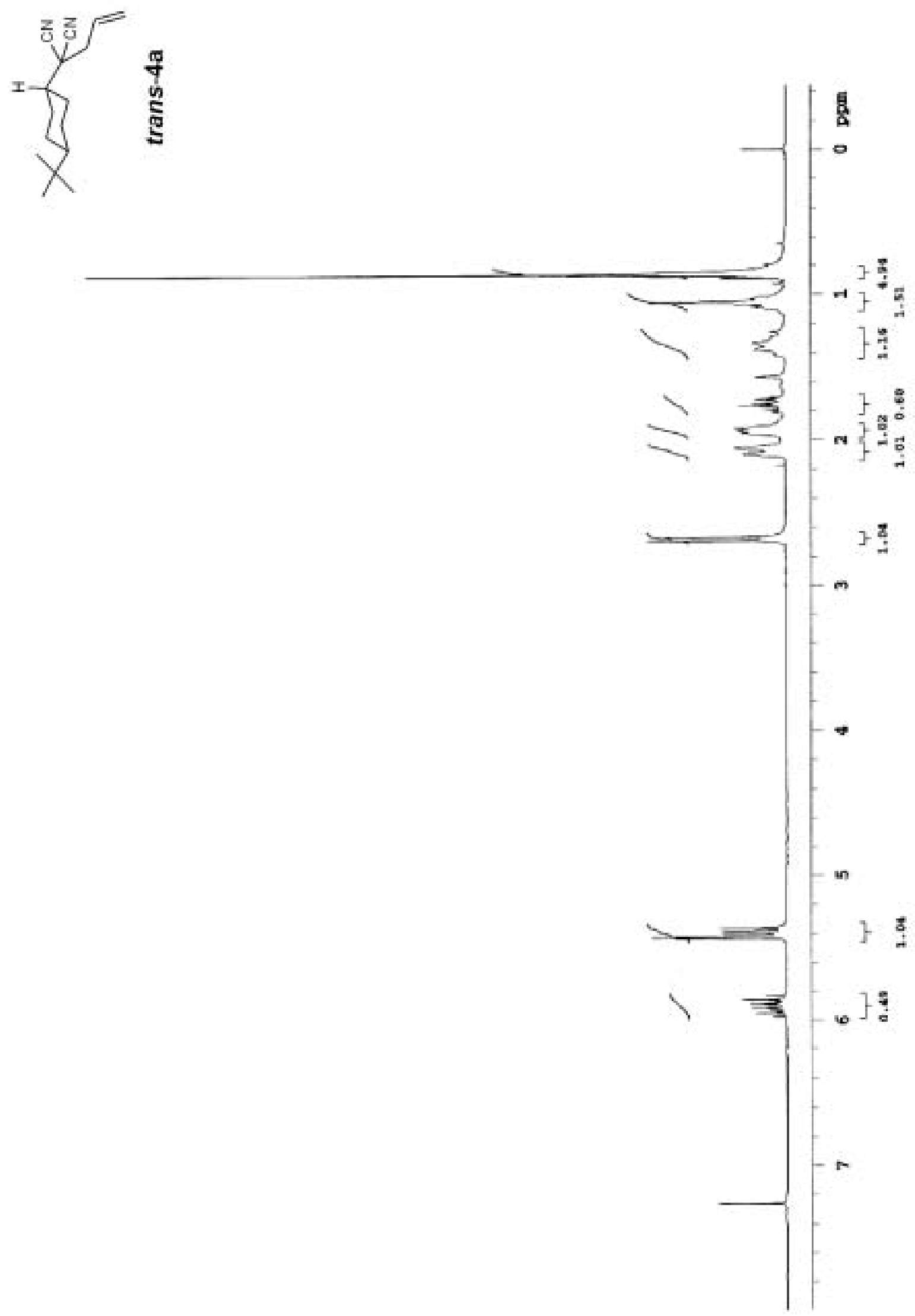

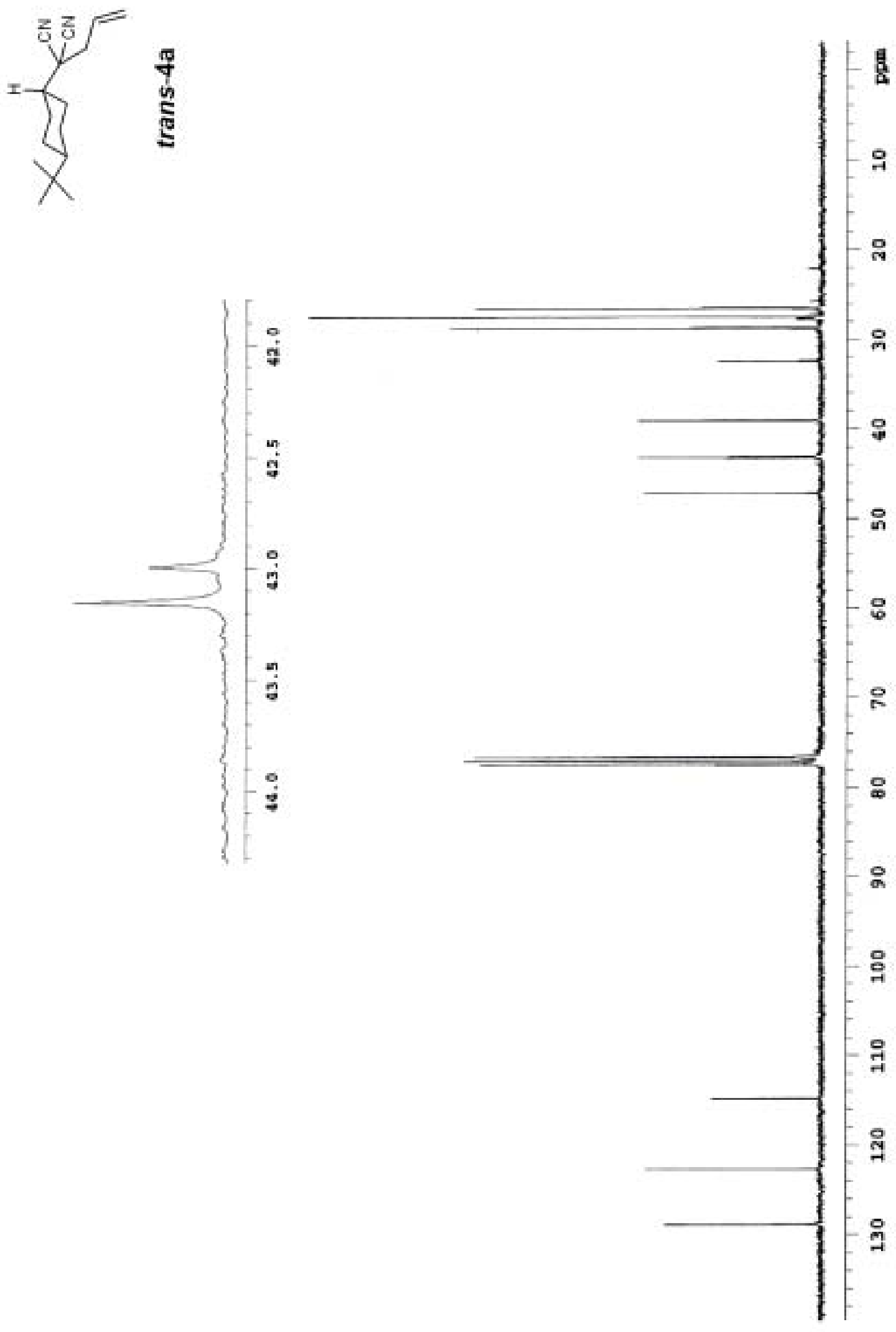

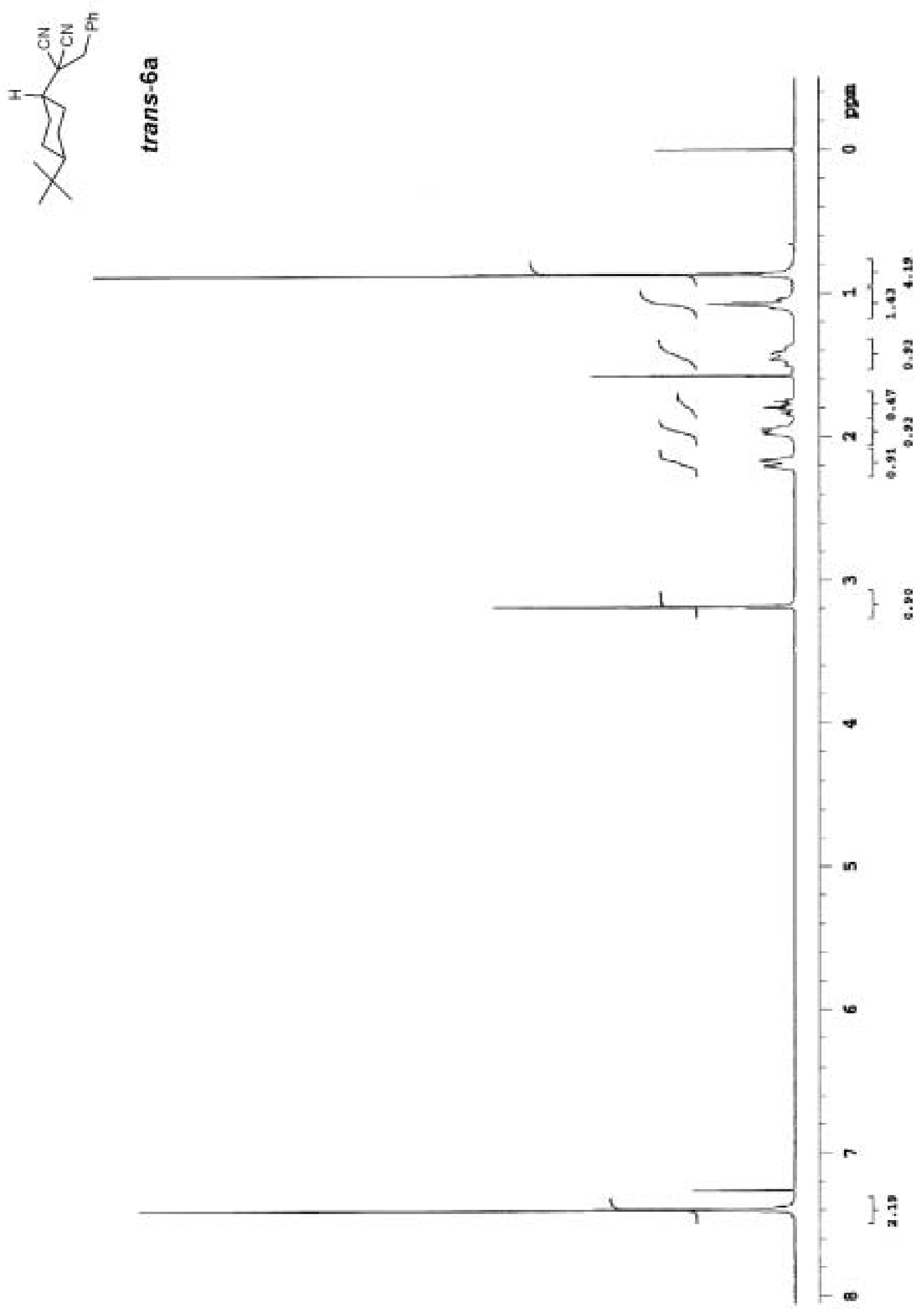

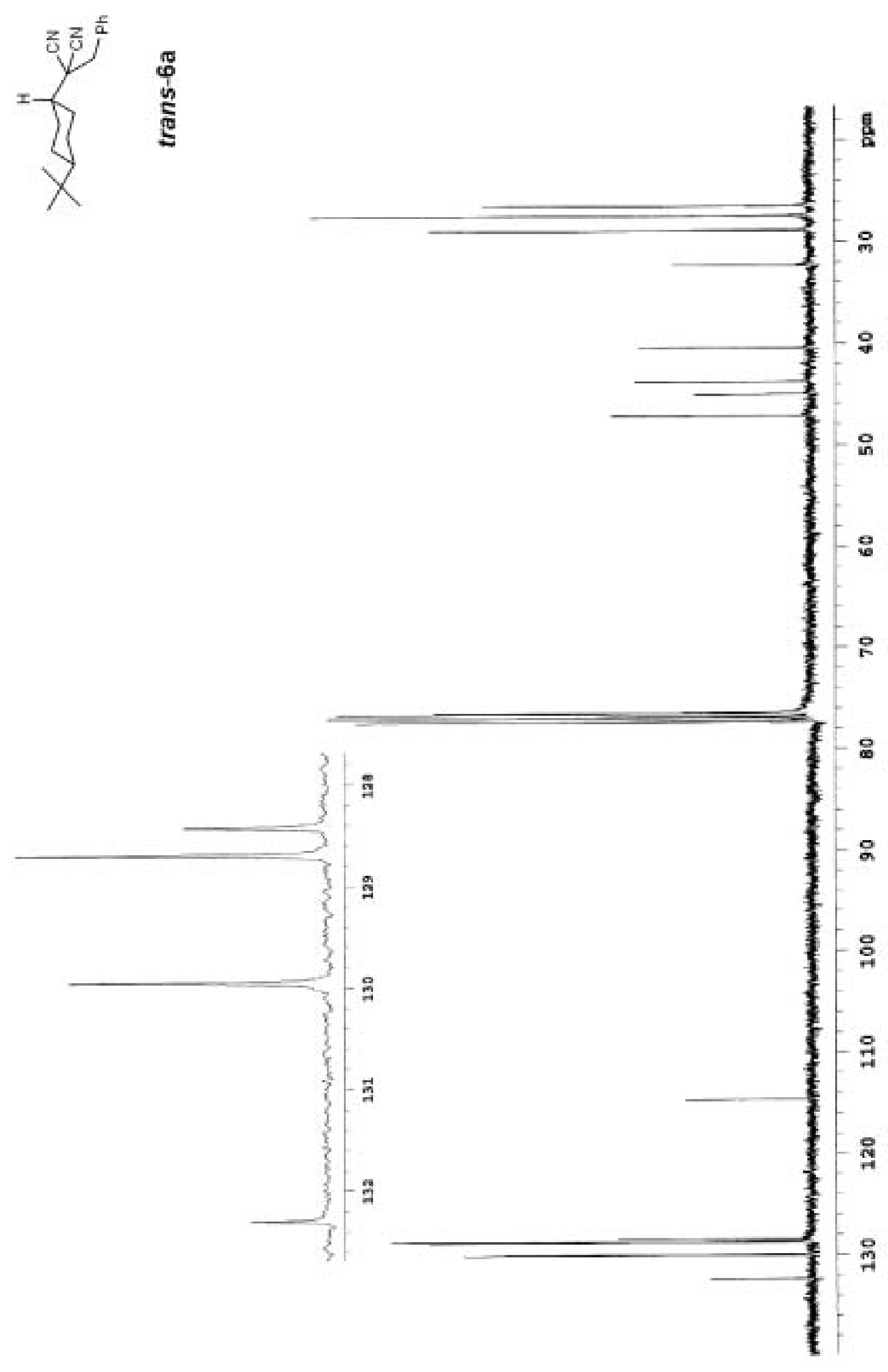

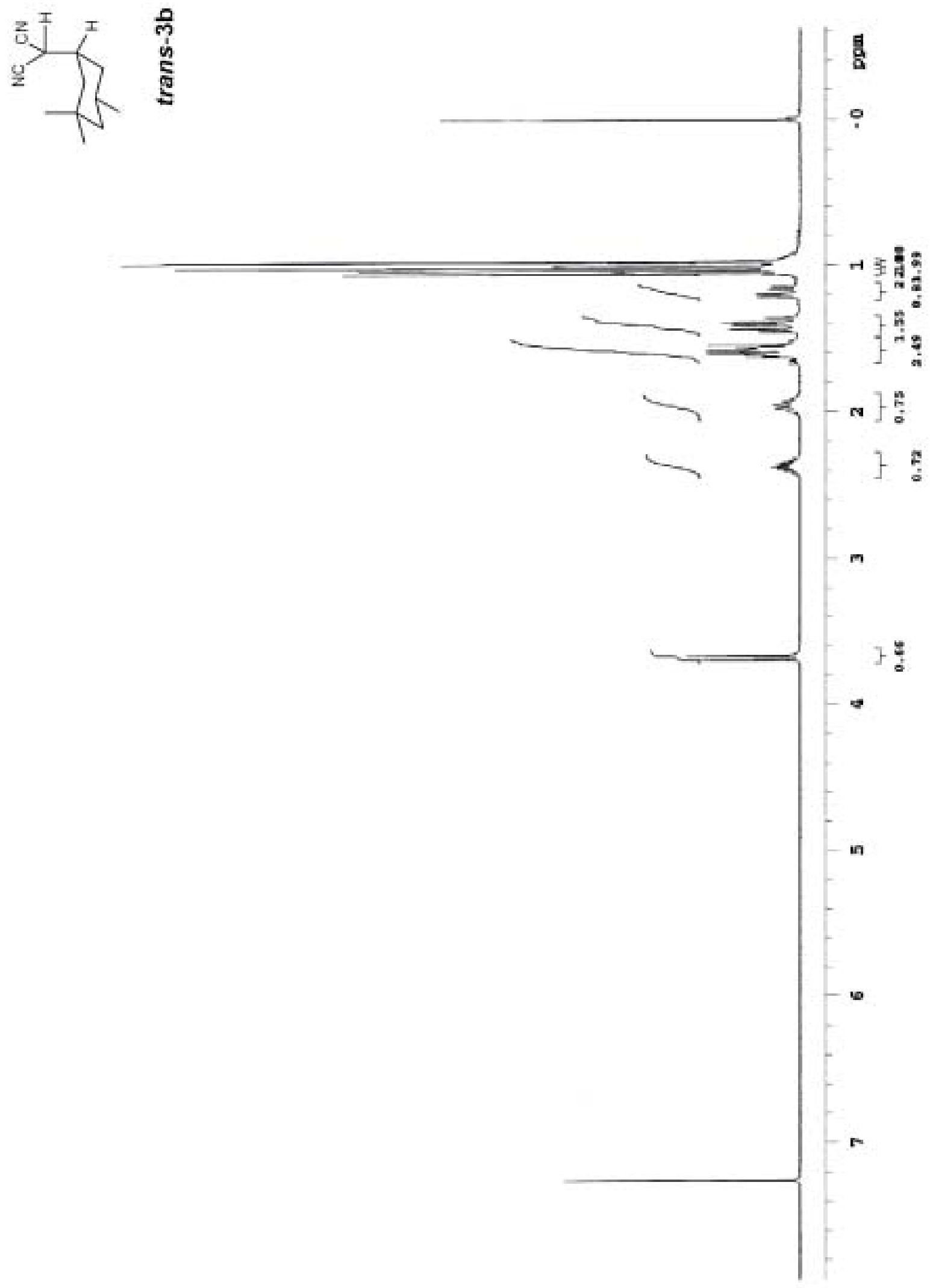

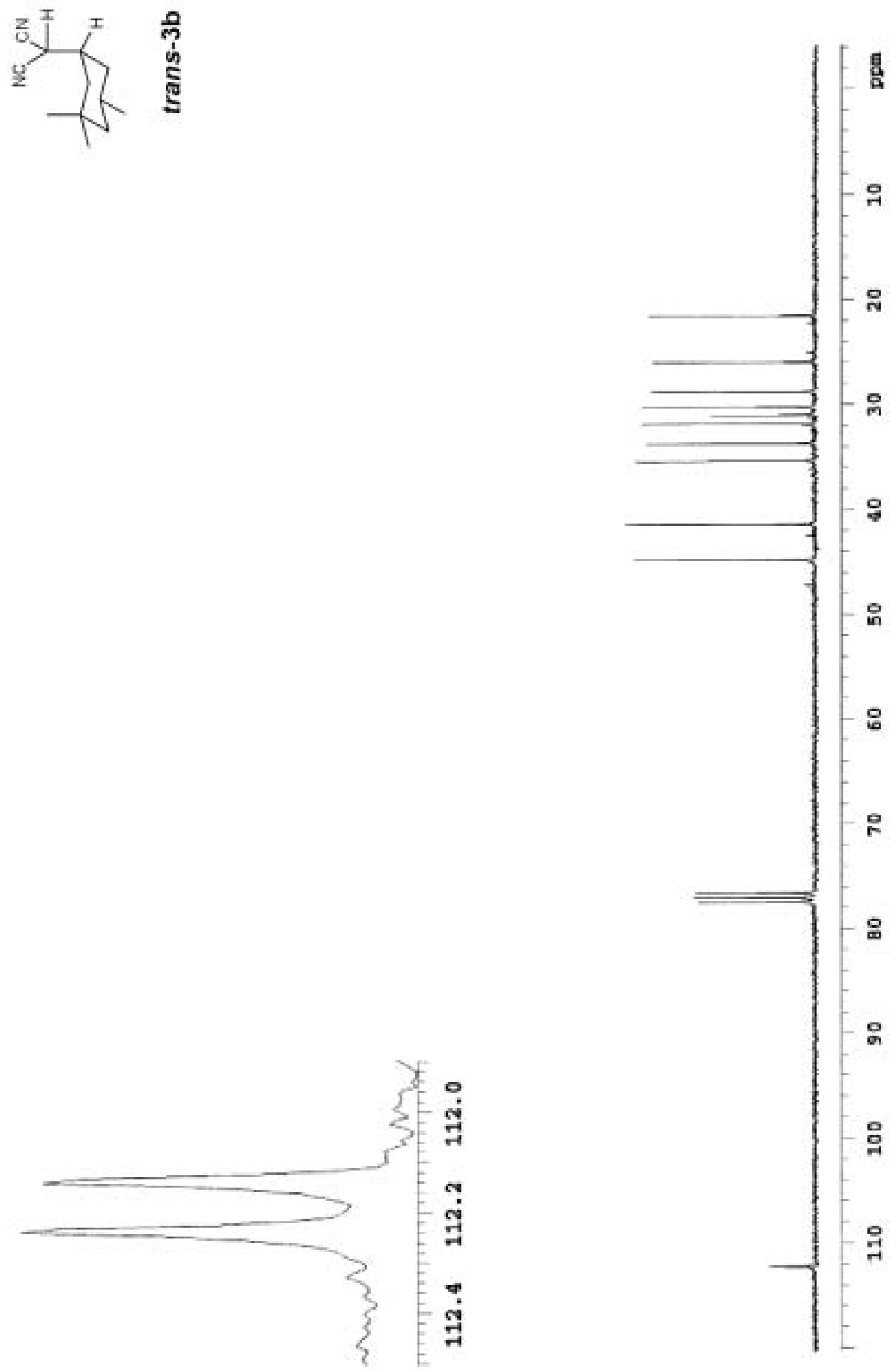
$\int_{2}^{\pi / 2}$ के

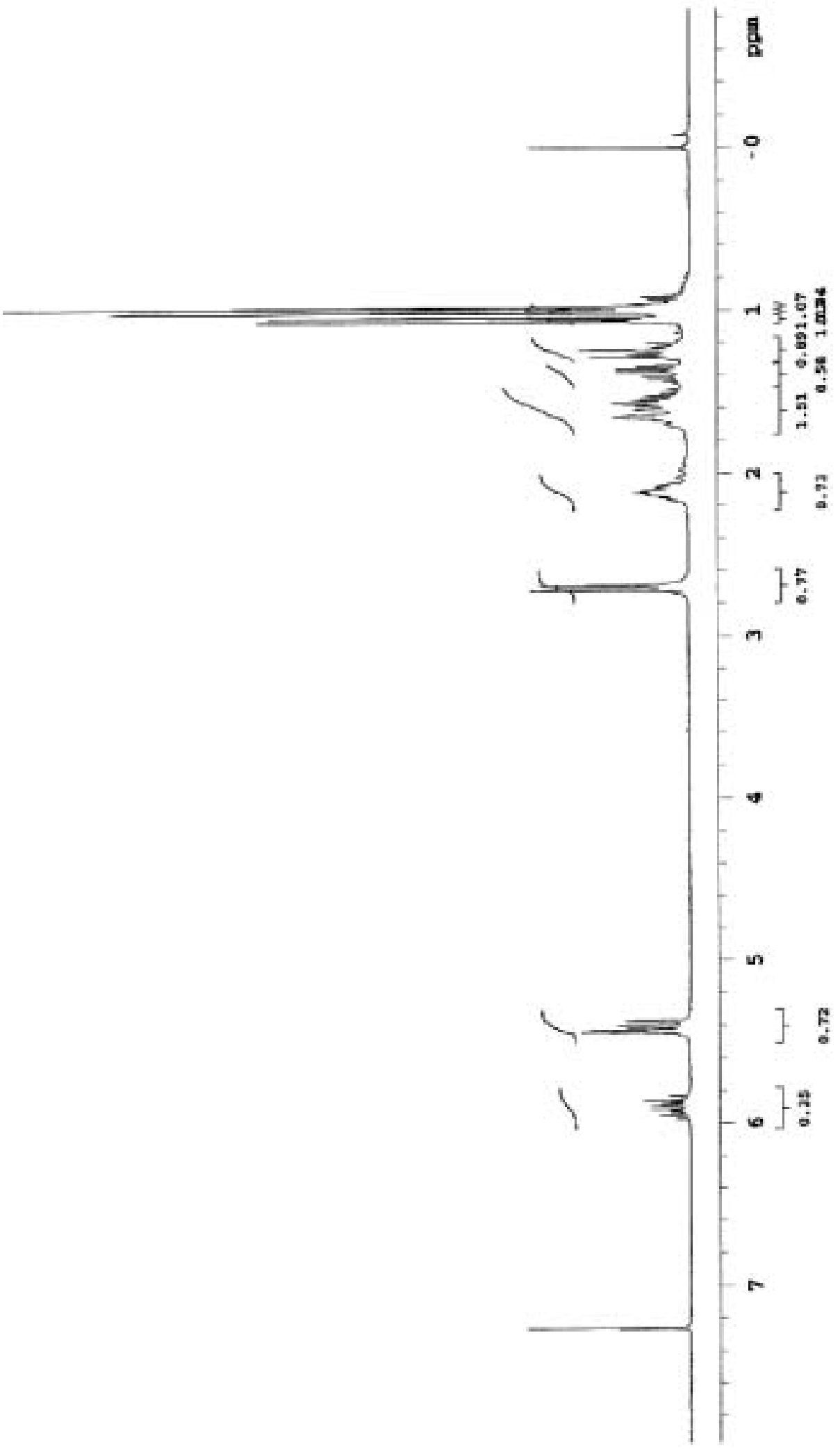



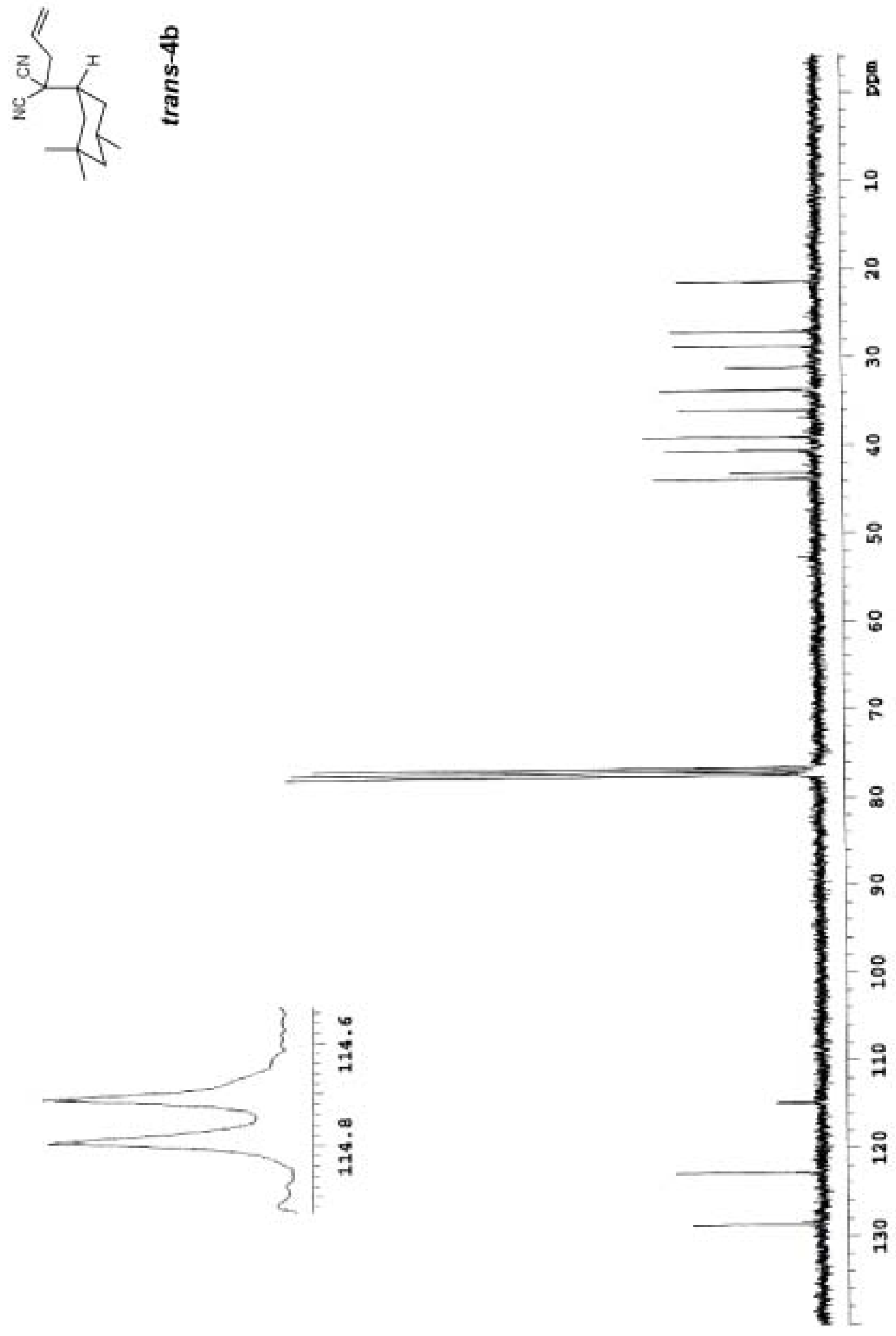


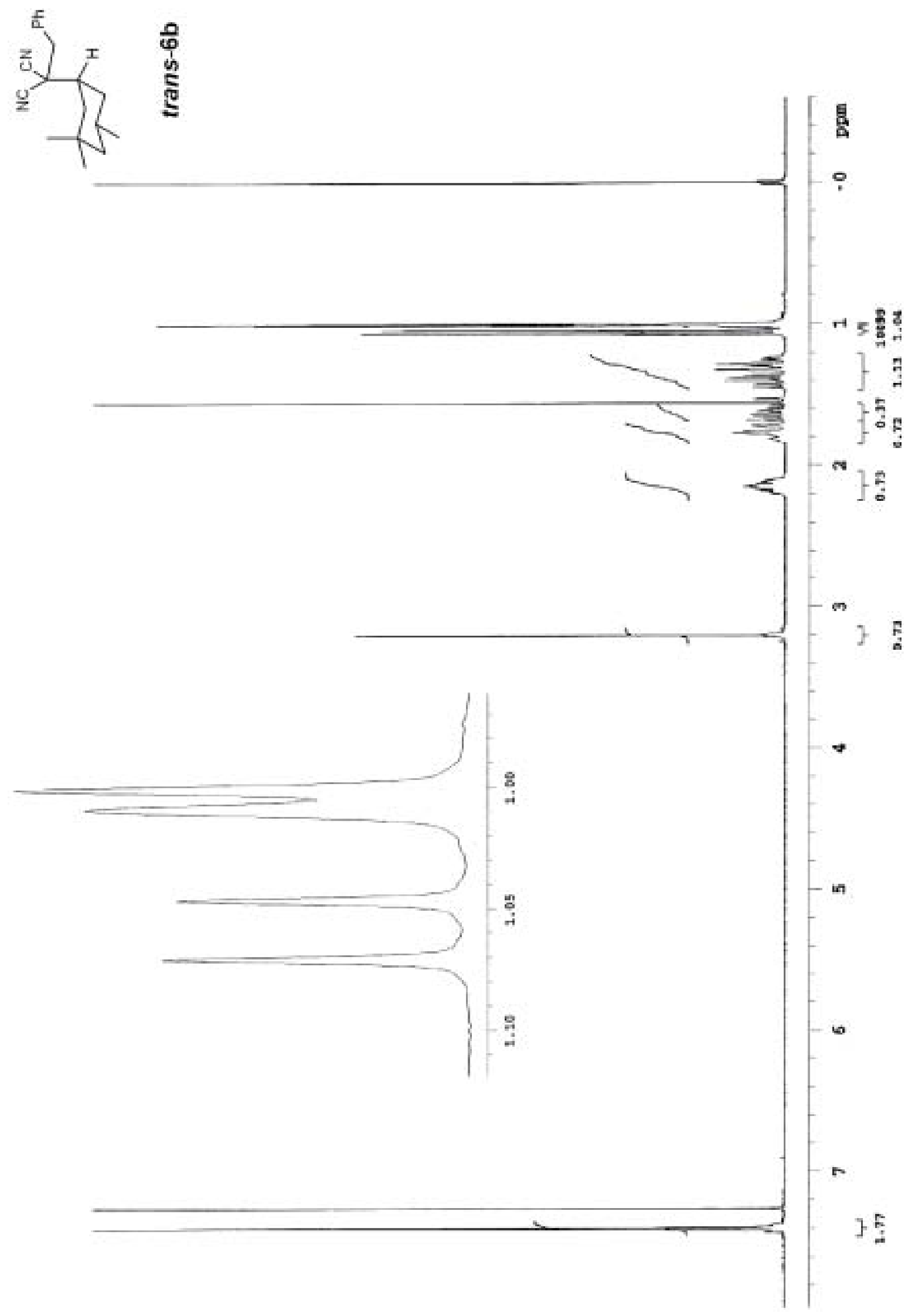



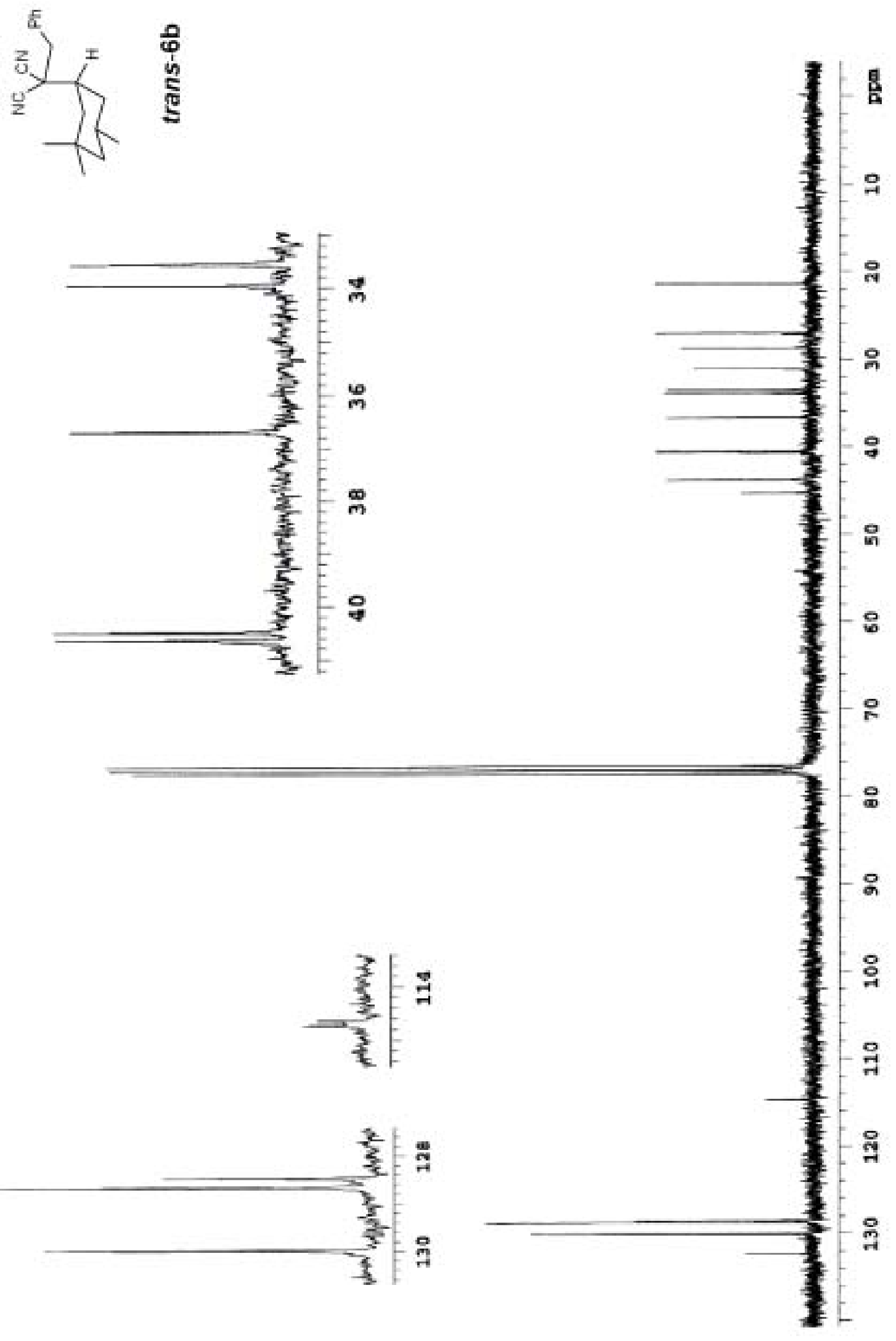


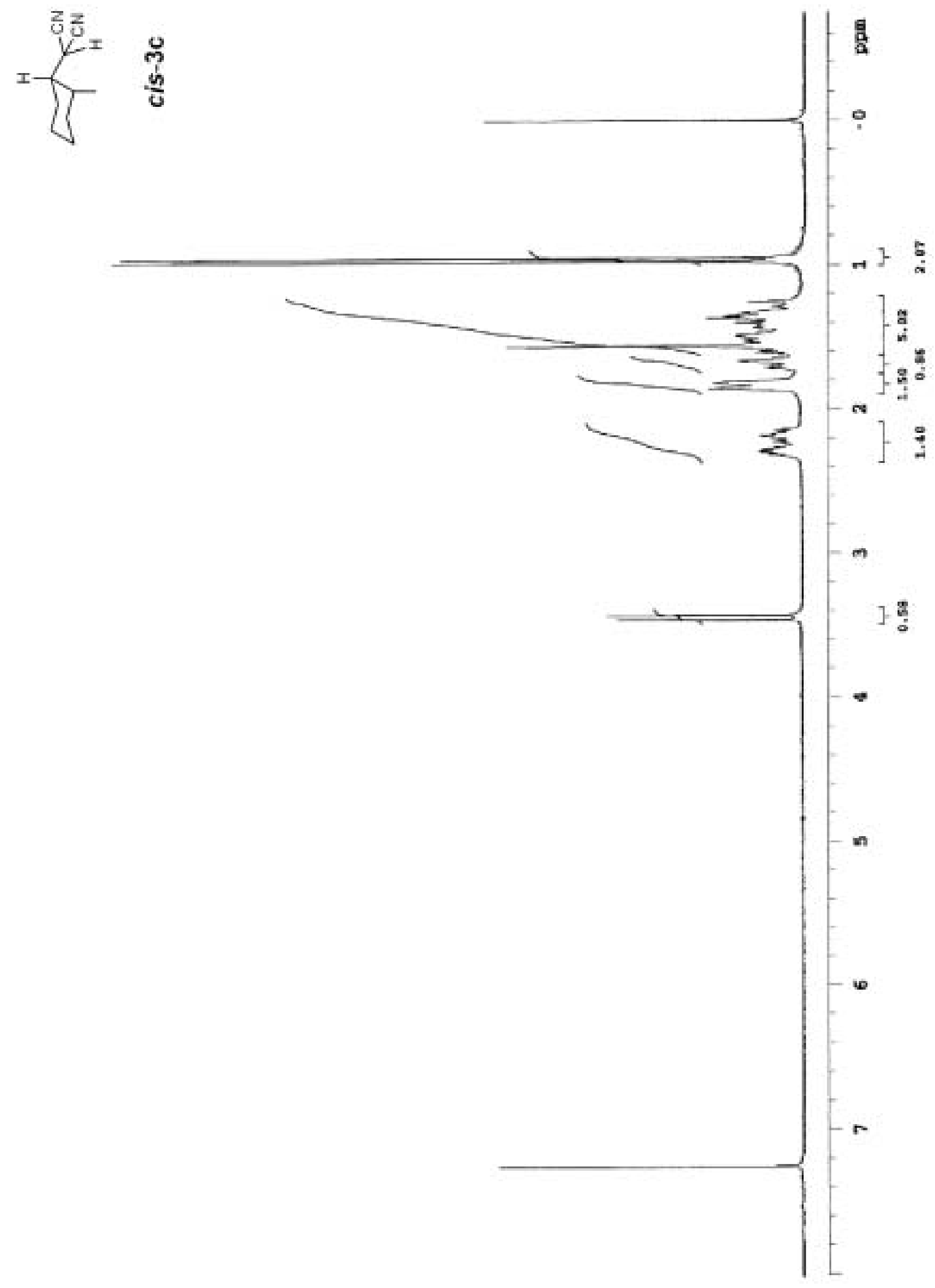




$$
\text { " }
$$




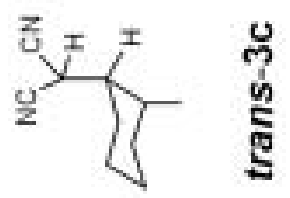

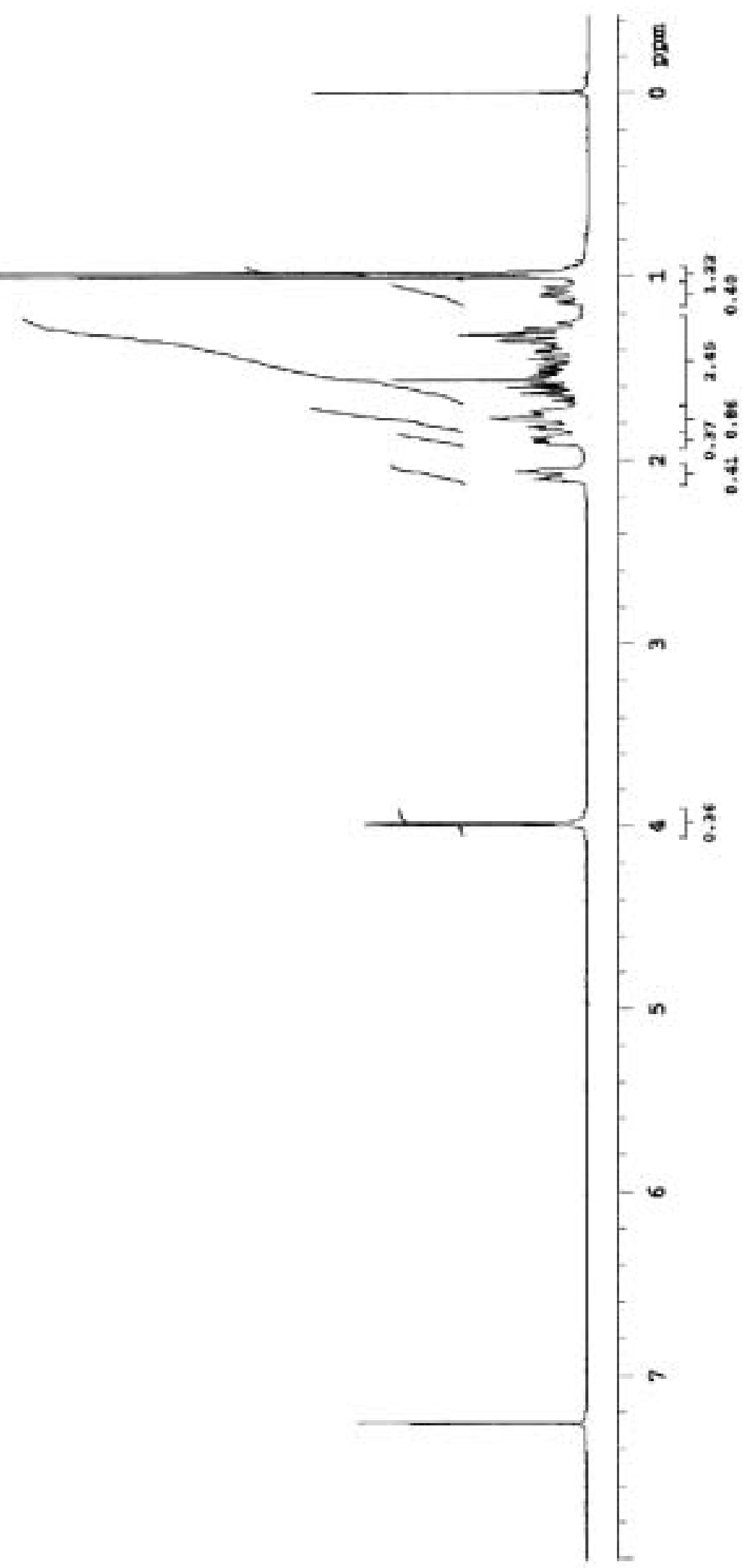




$$
{ }^{2}{ }^{I}
$$
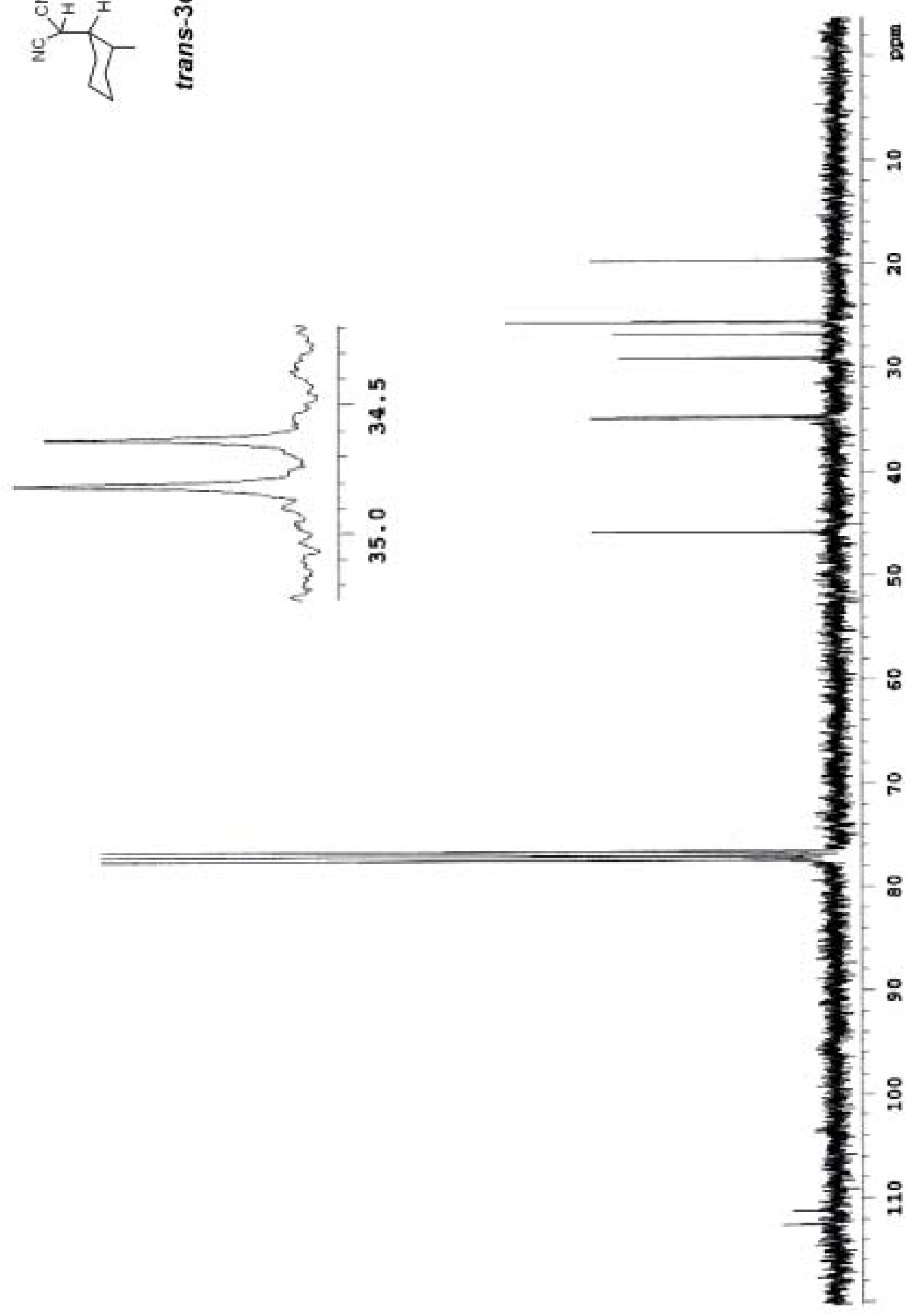


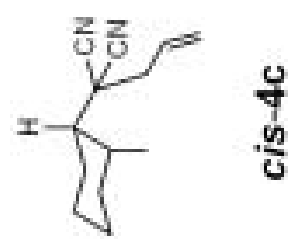

$\frac{y}{4}$

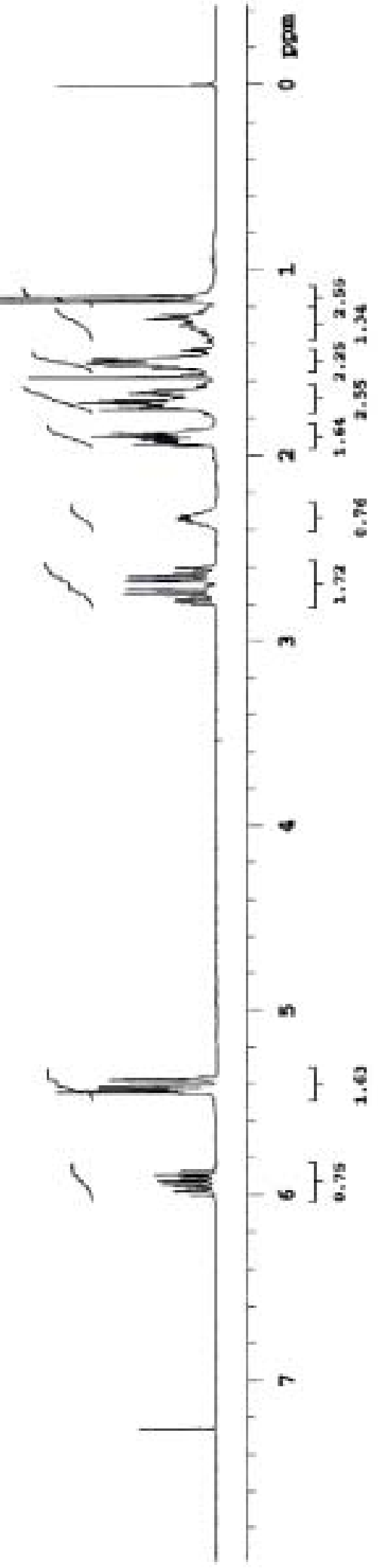



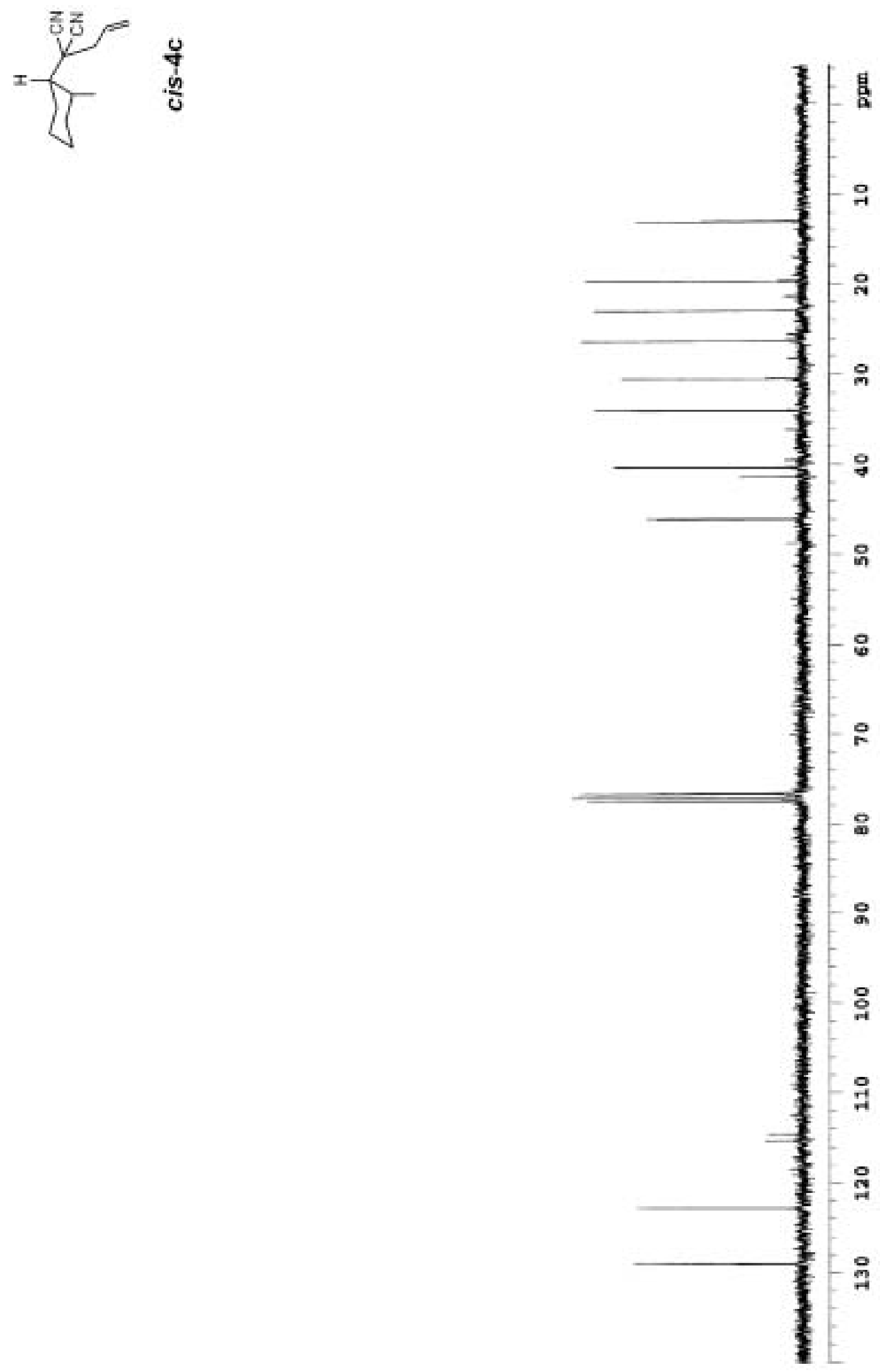

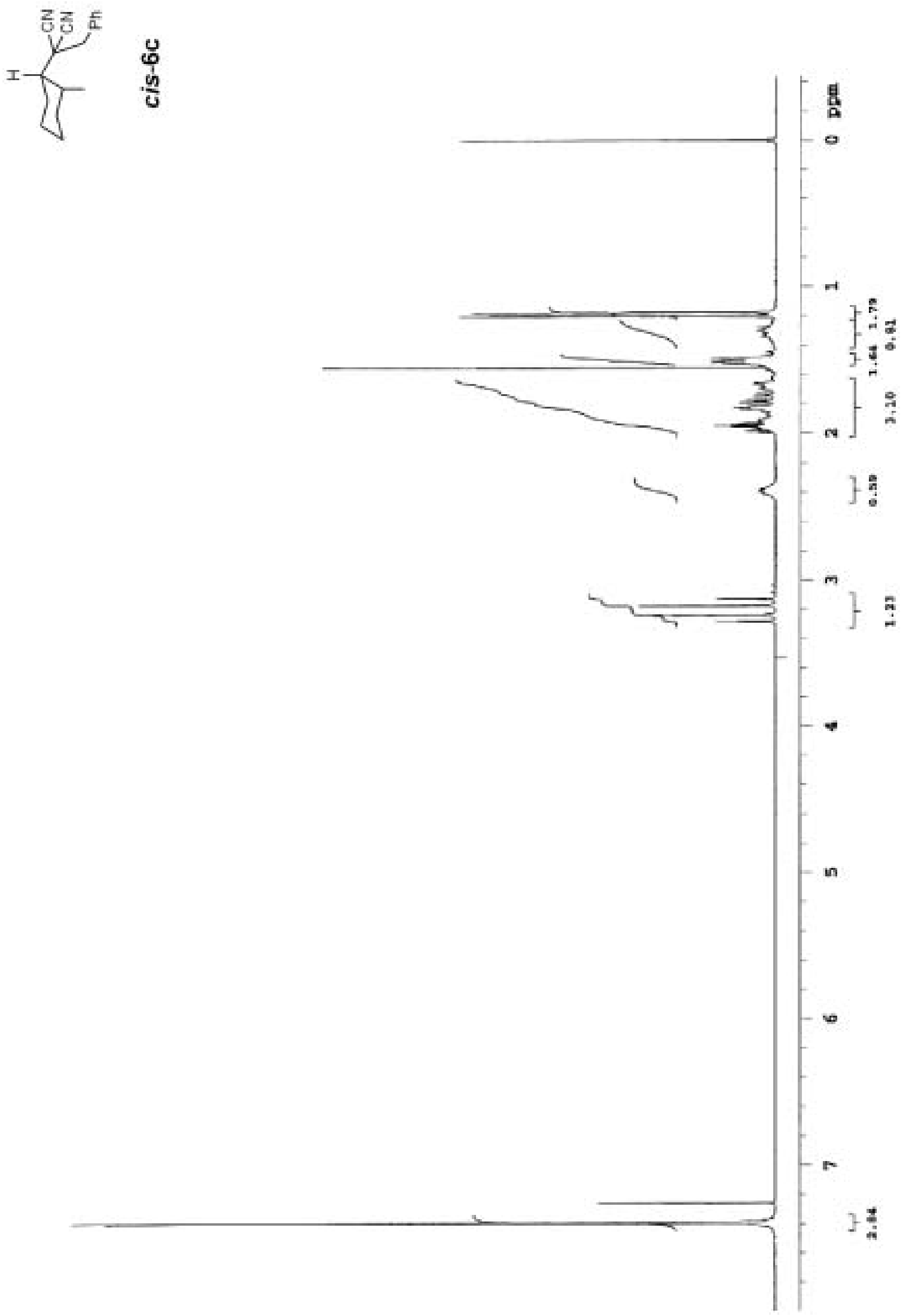


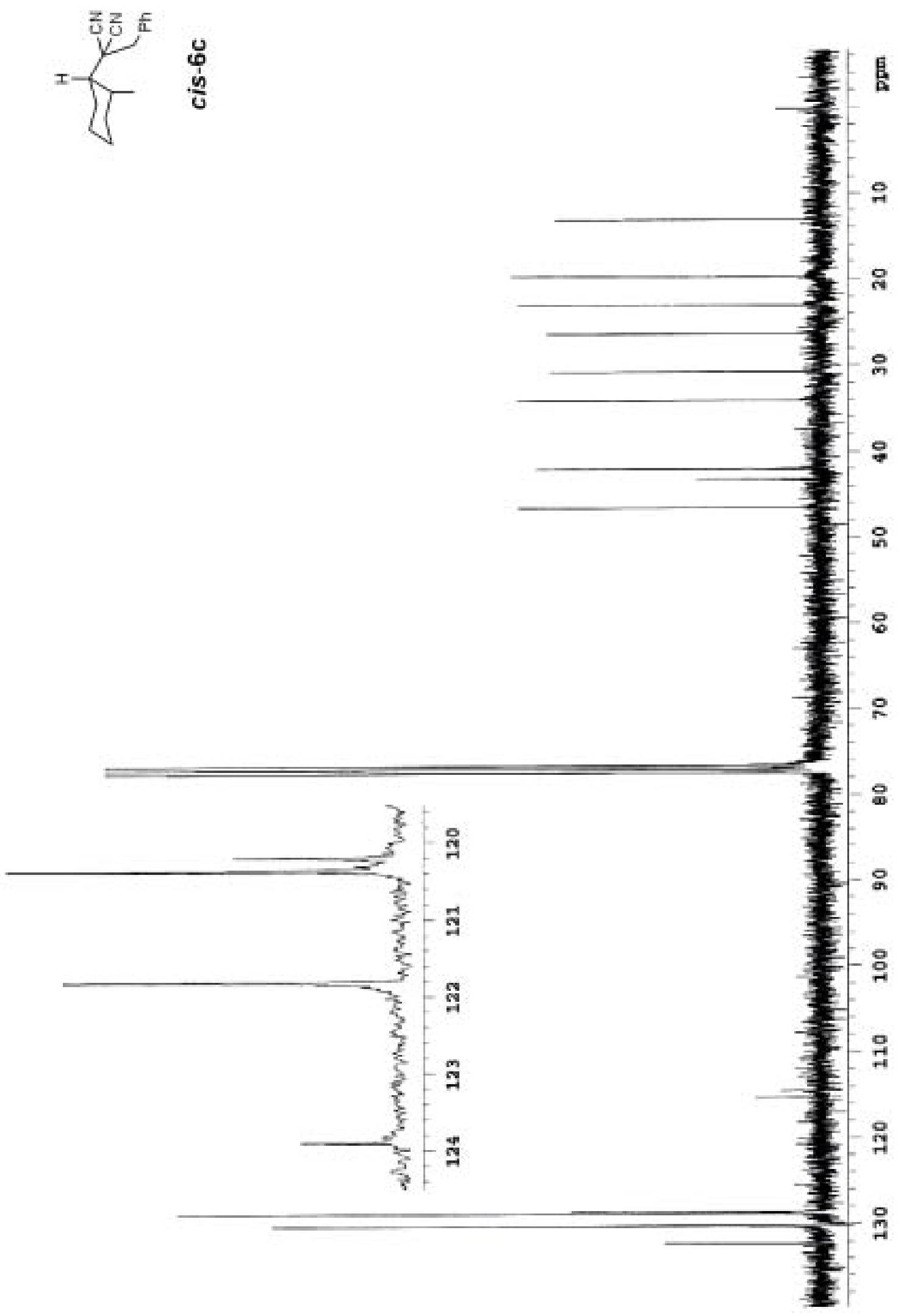




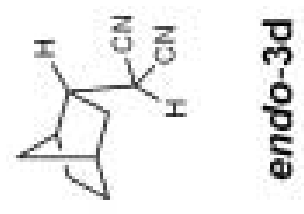

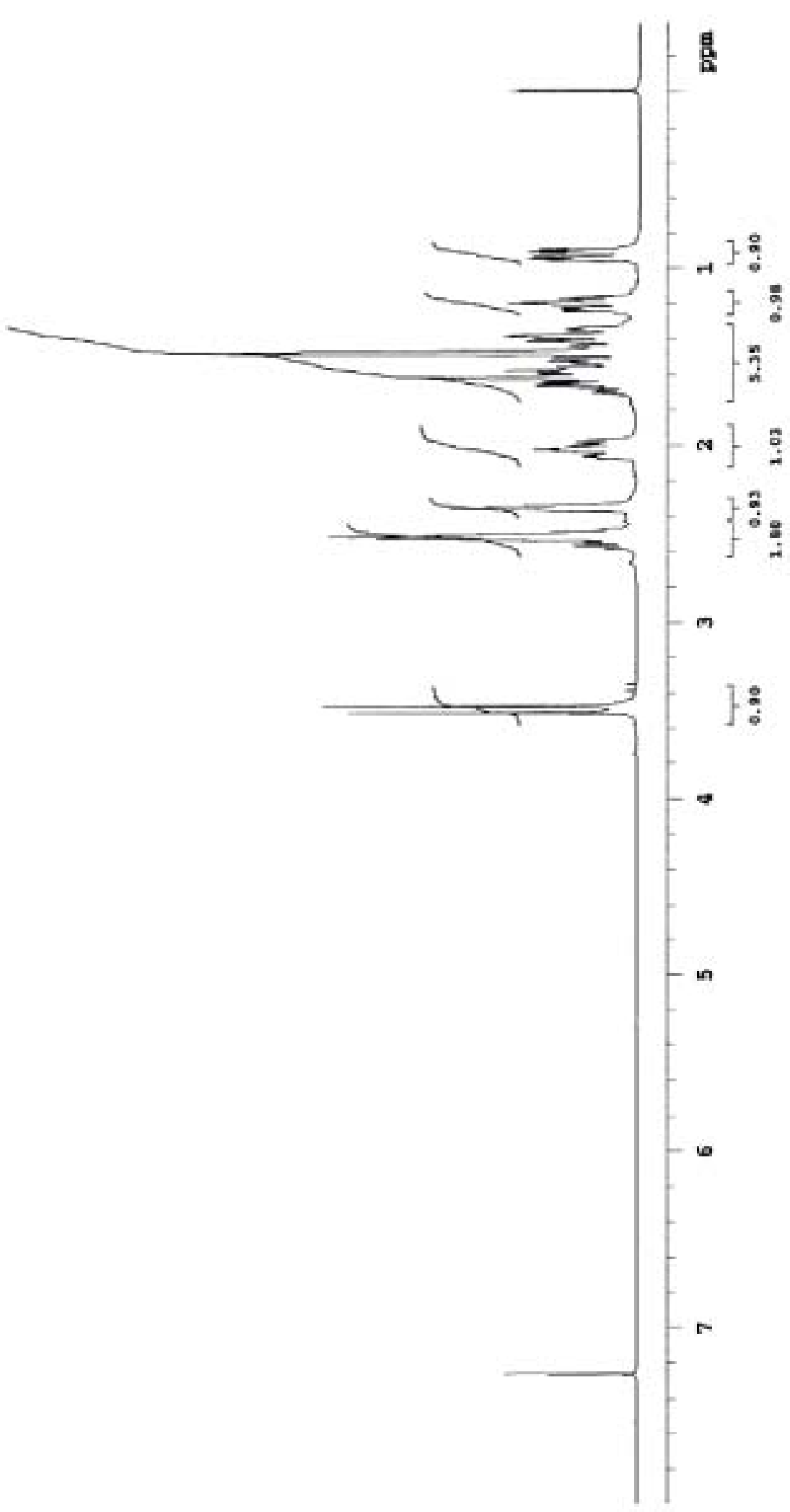



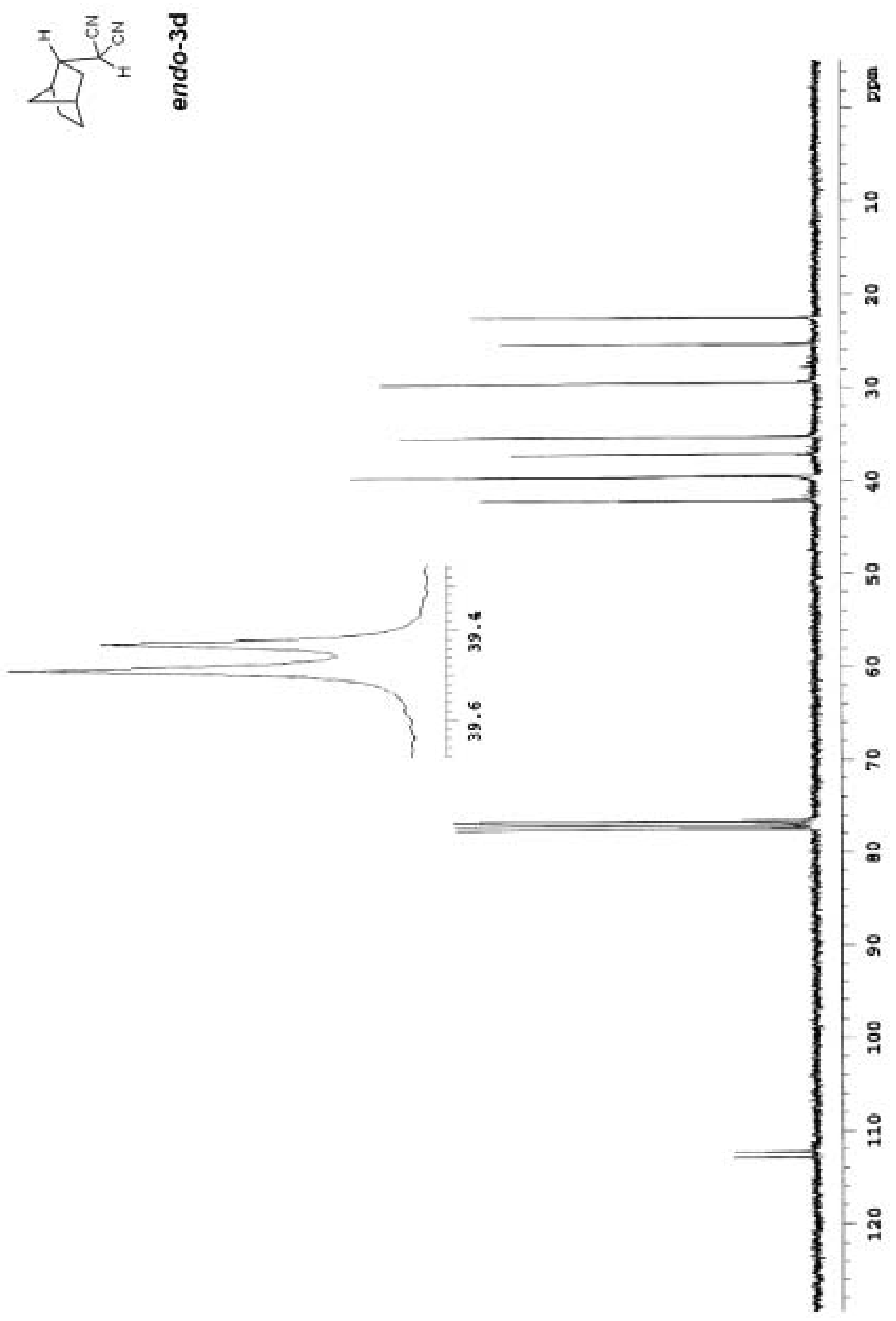

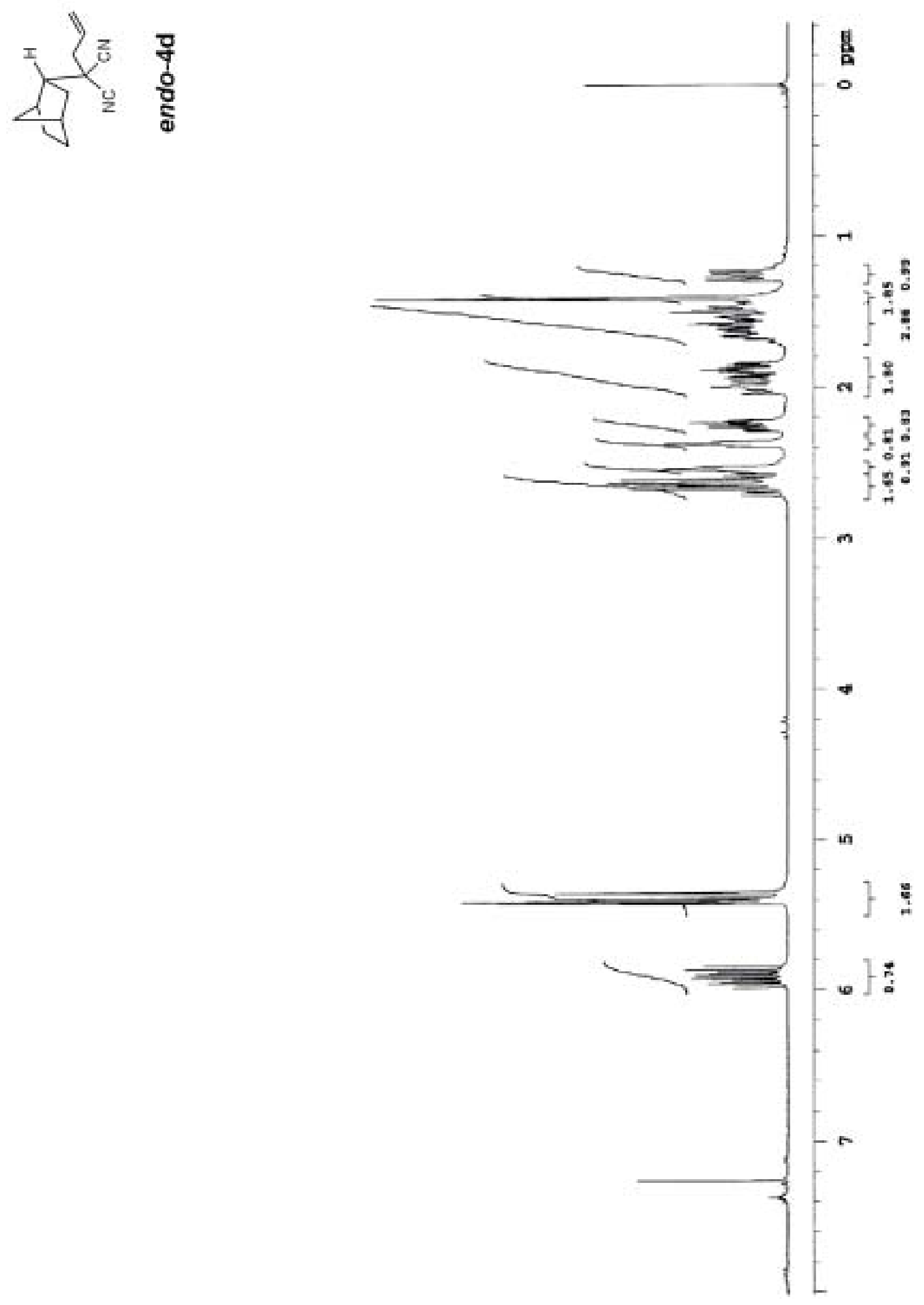


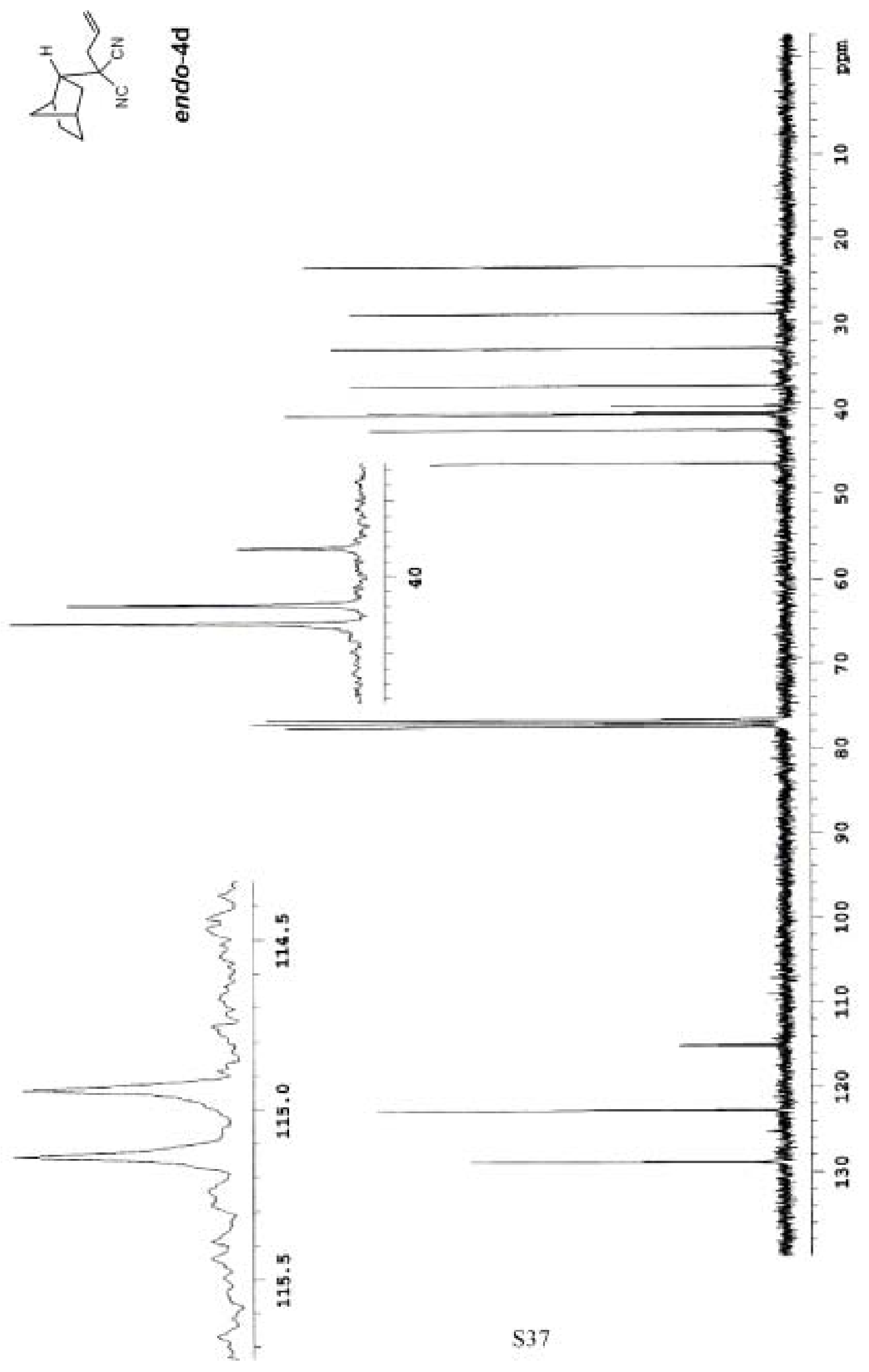



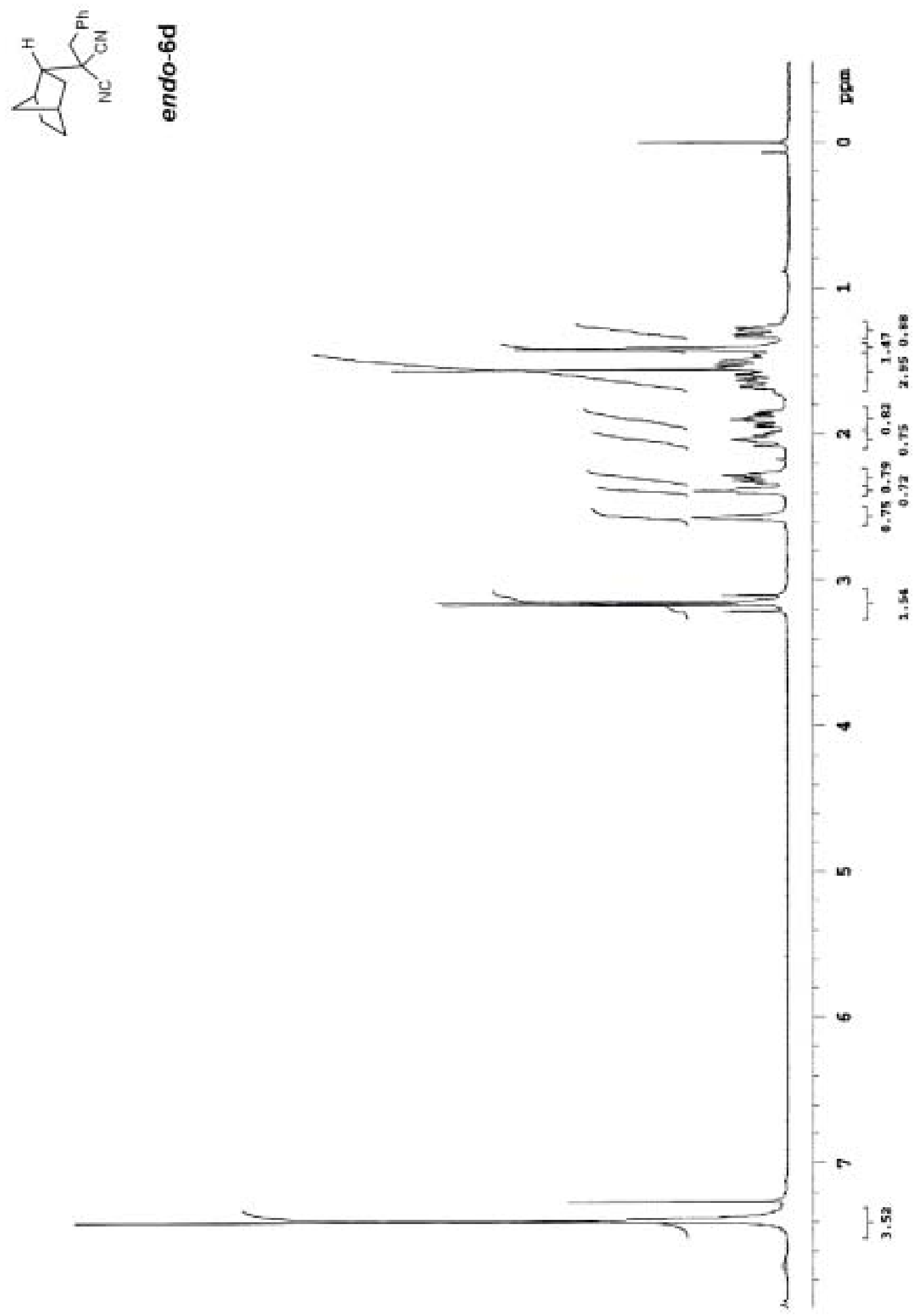

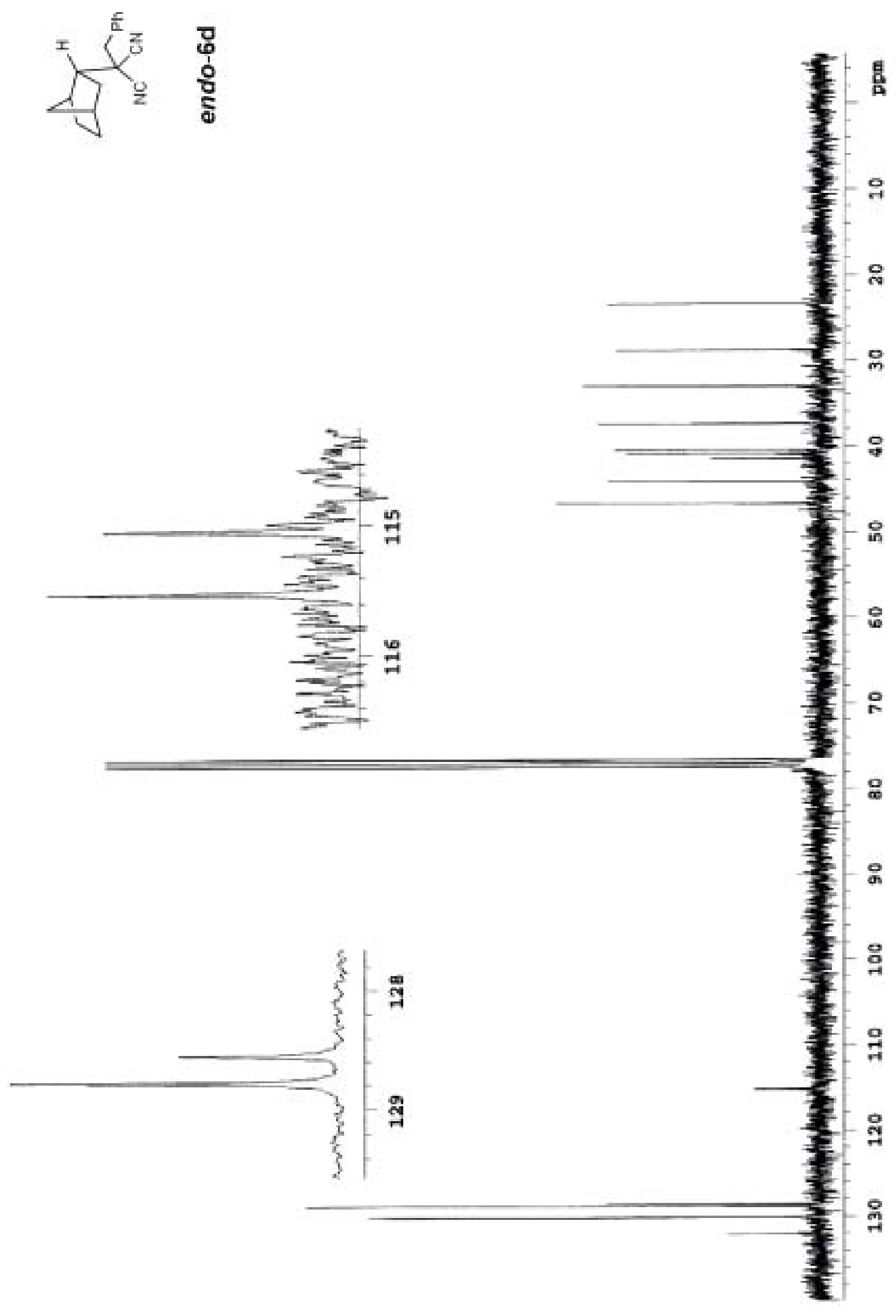

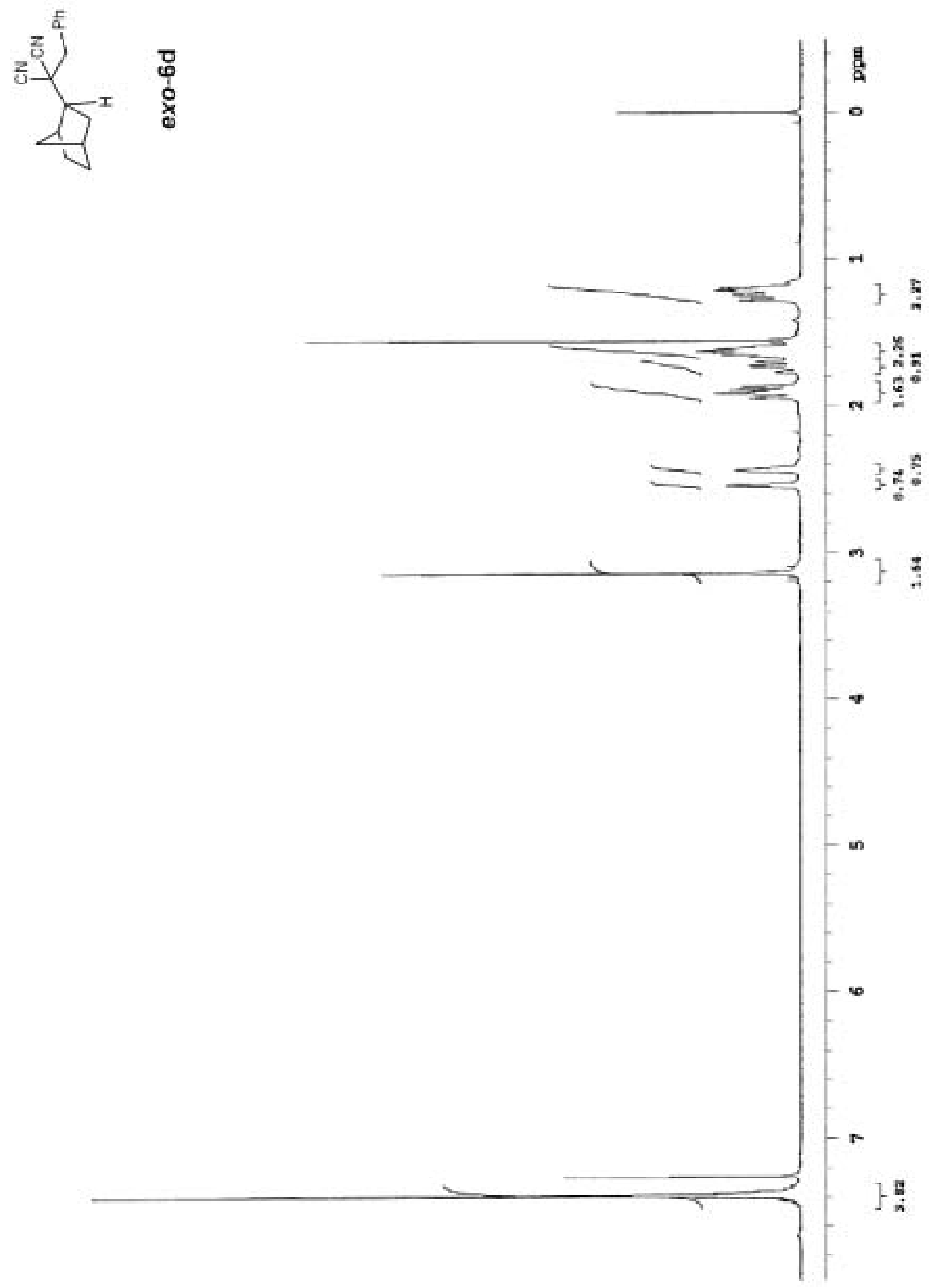


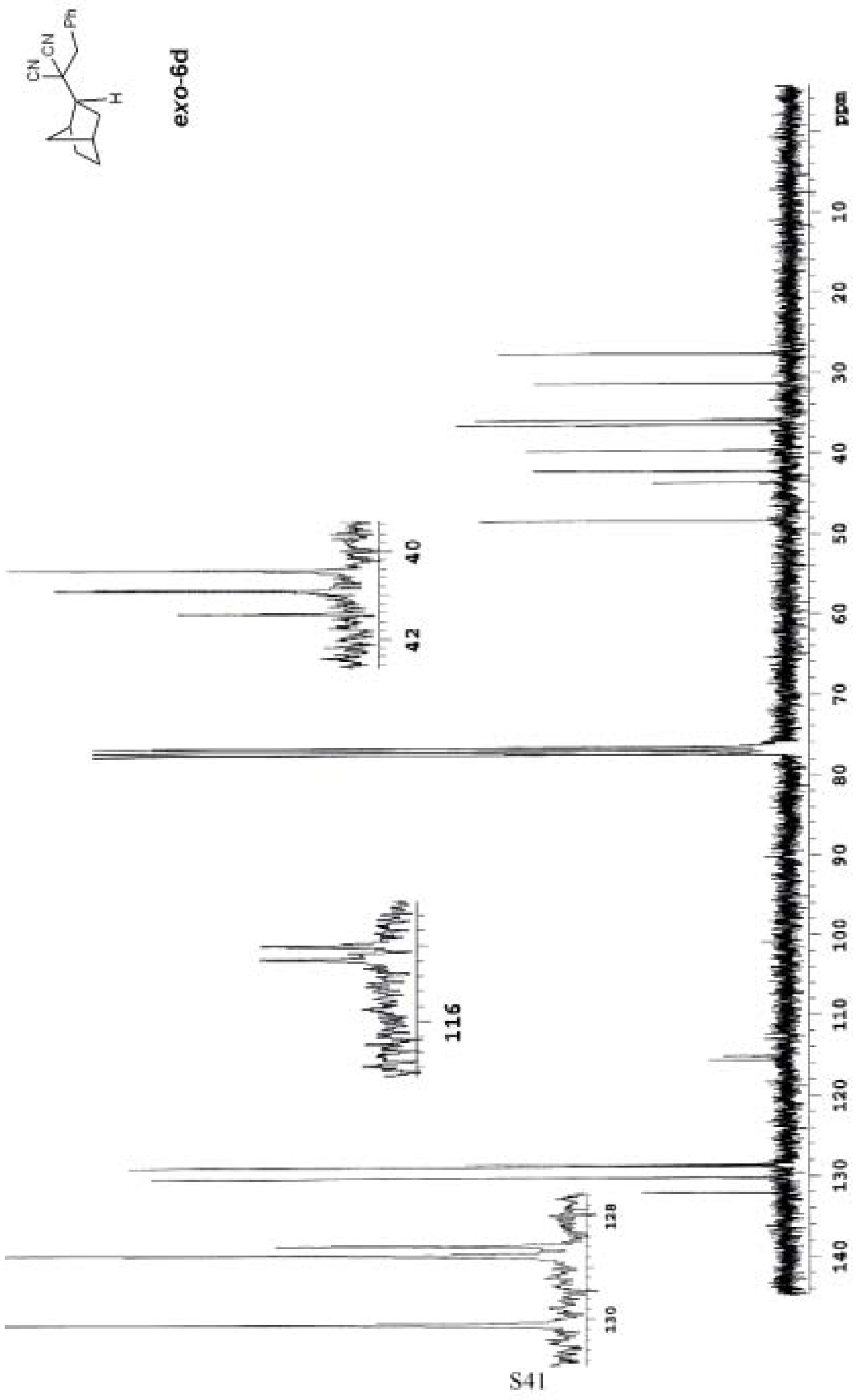




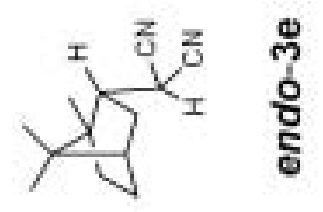

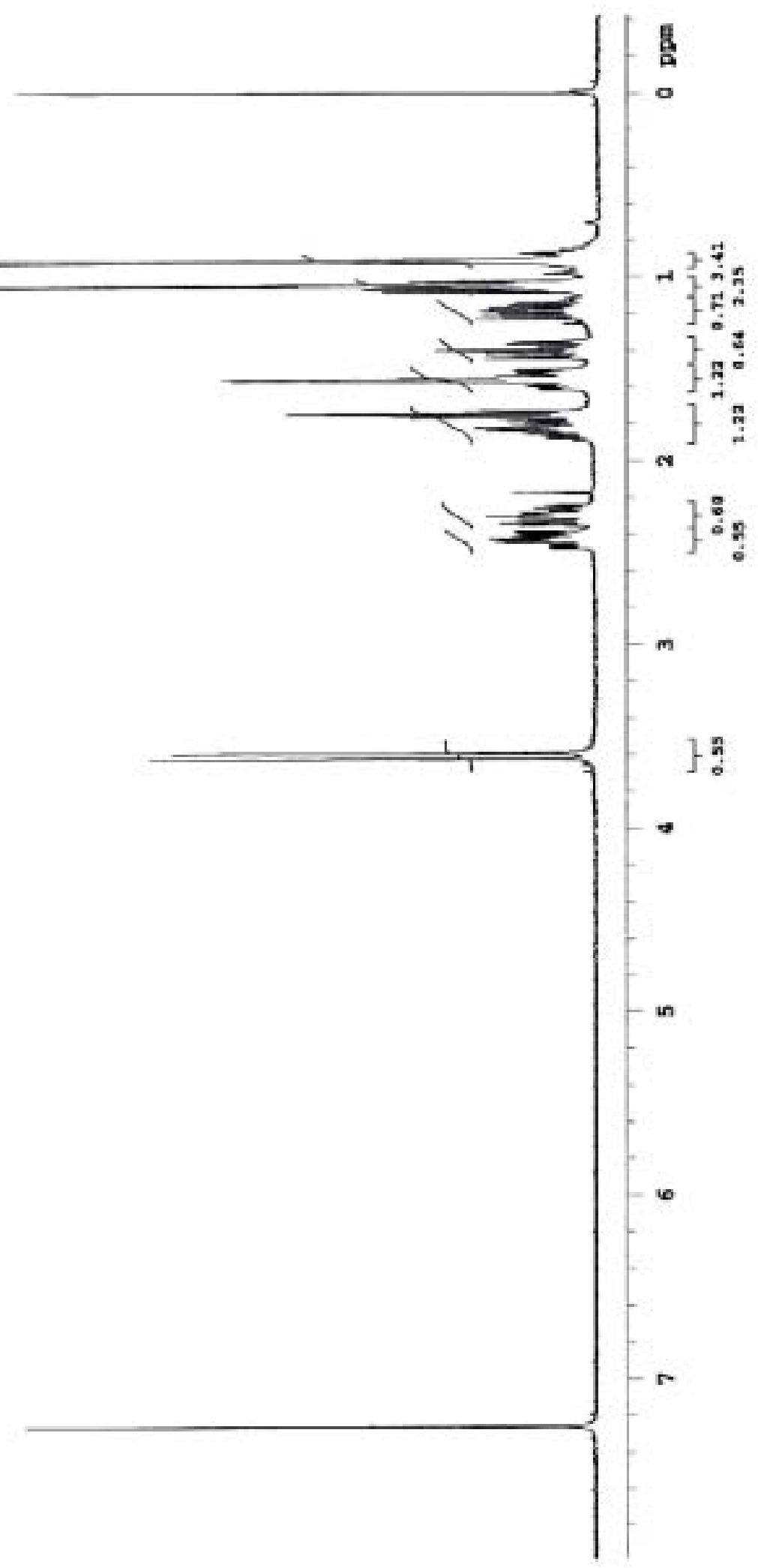


(1)

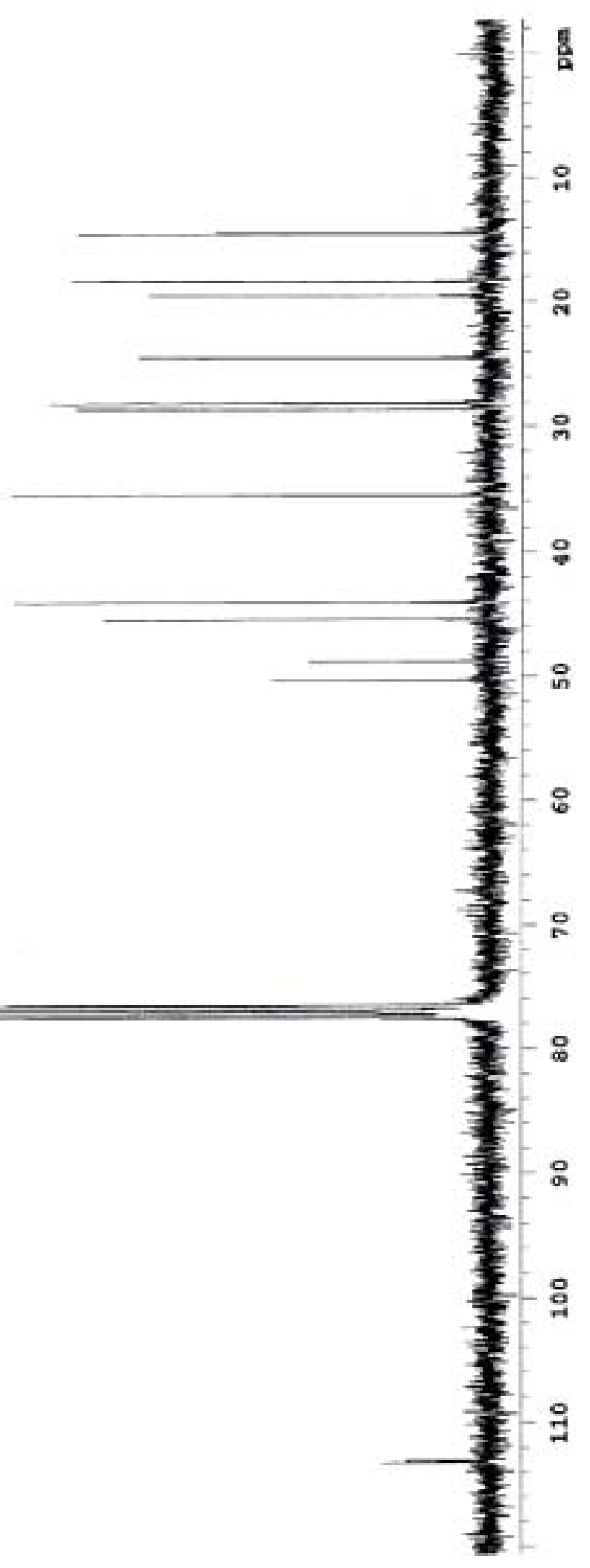


$\sum^{I}$

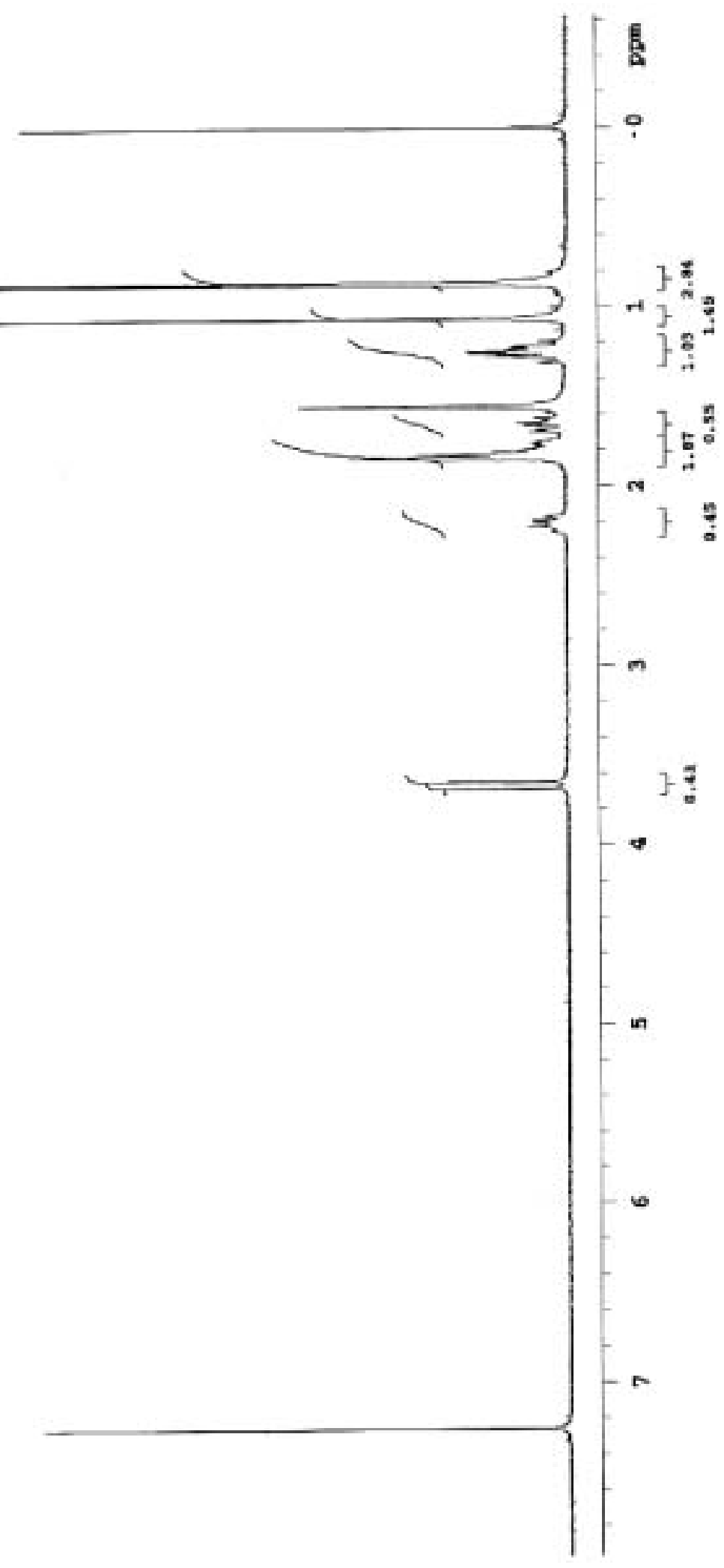




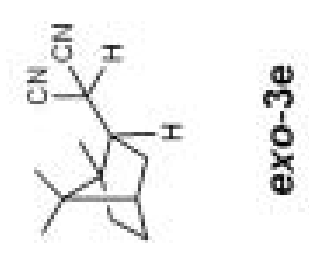

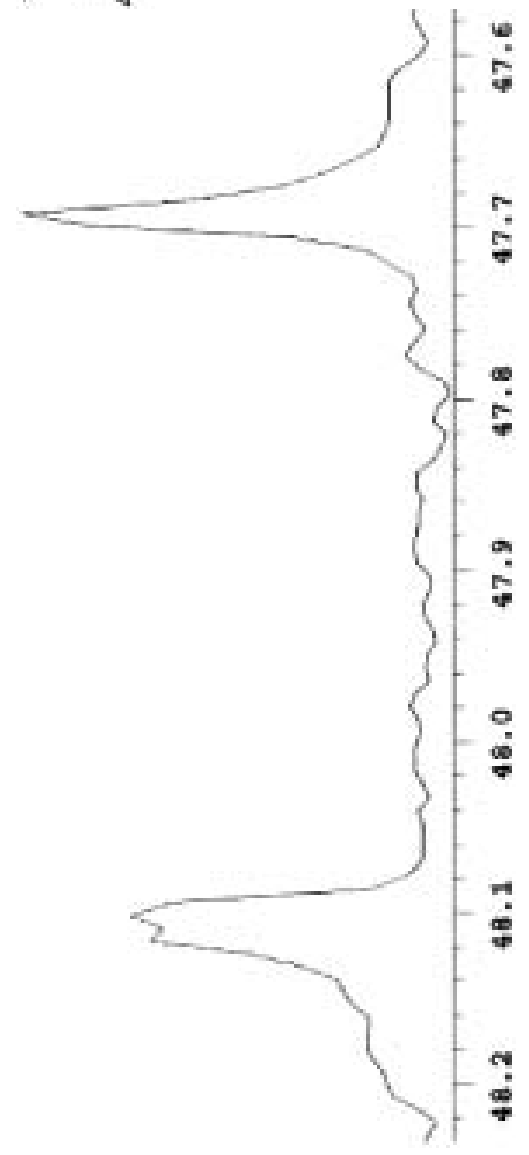

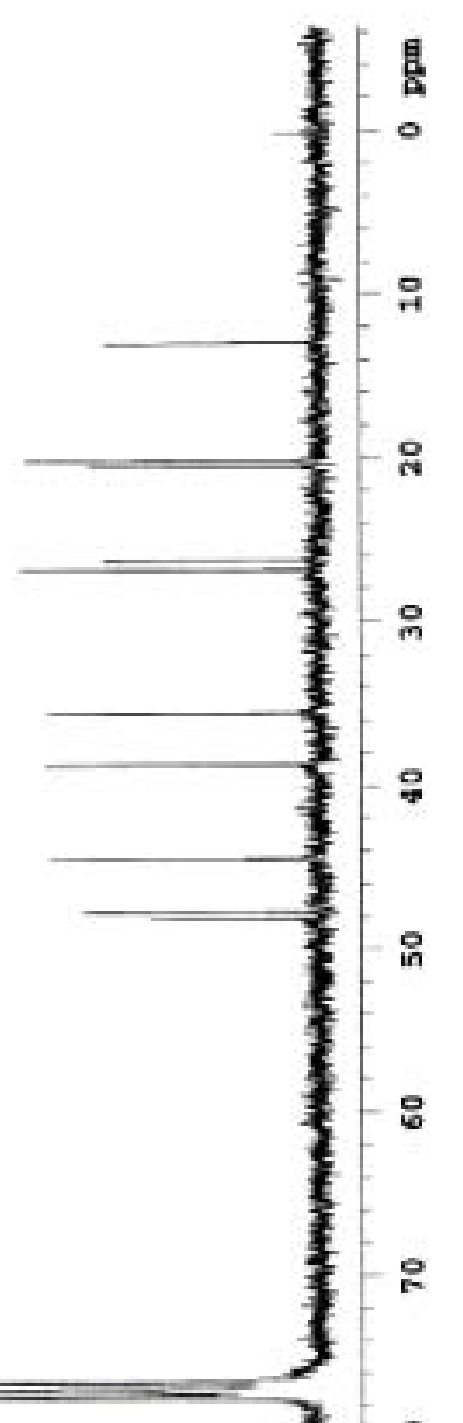

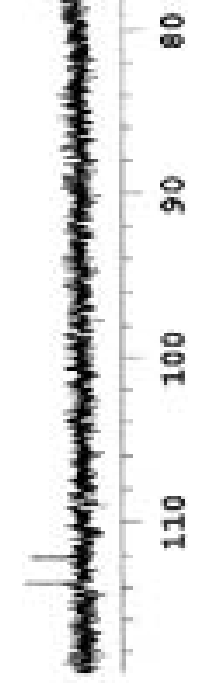


강

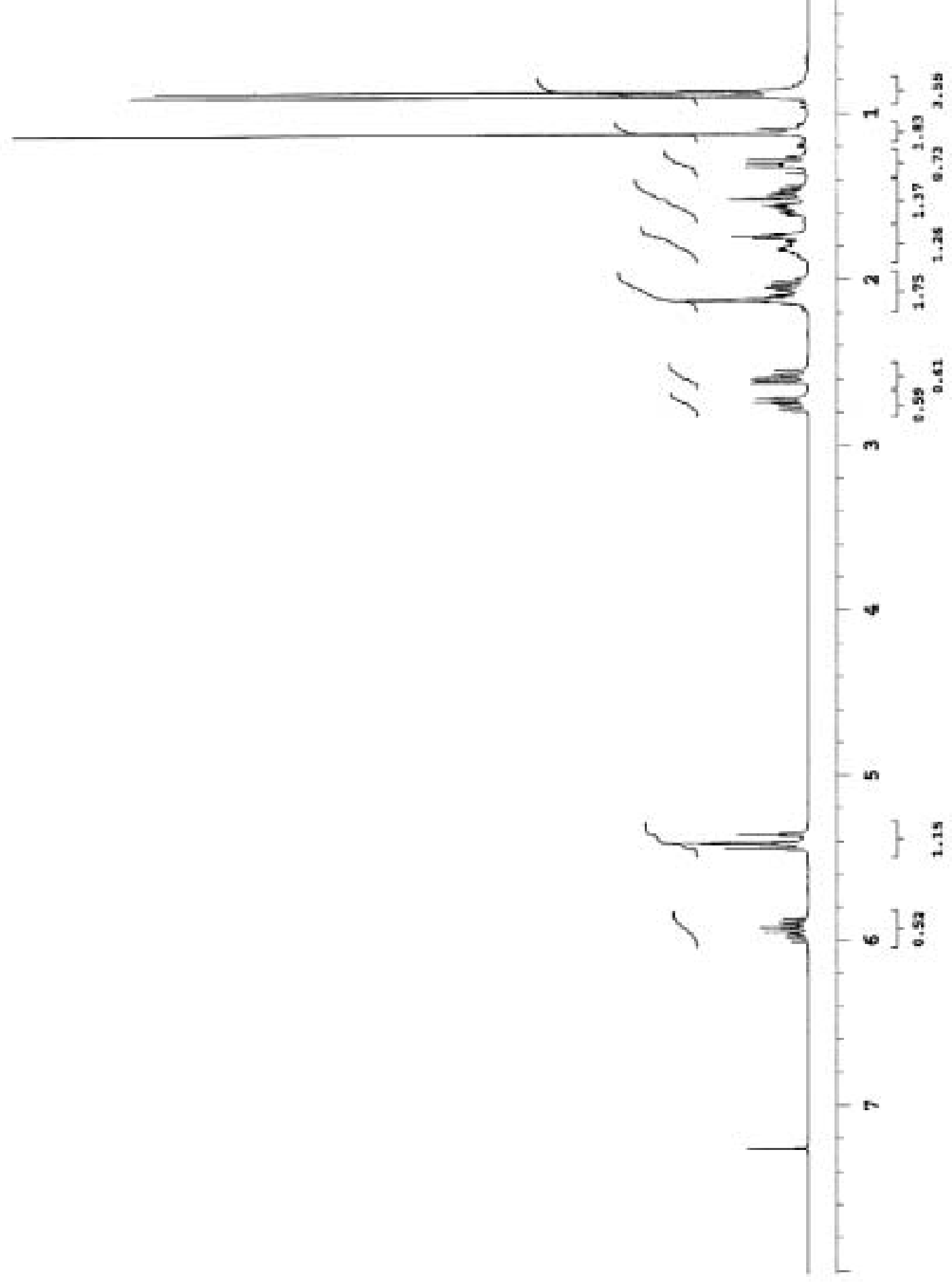



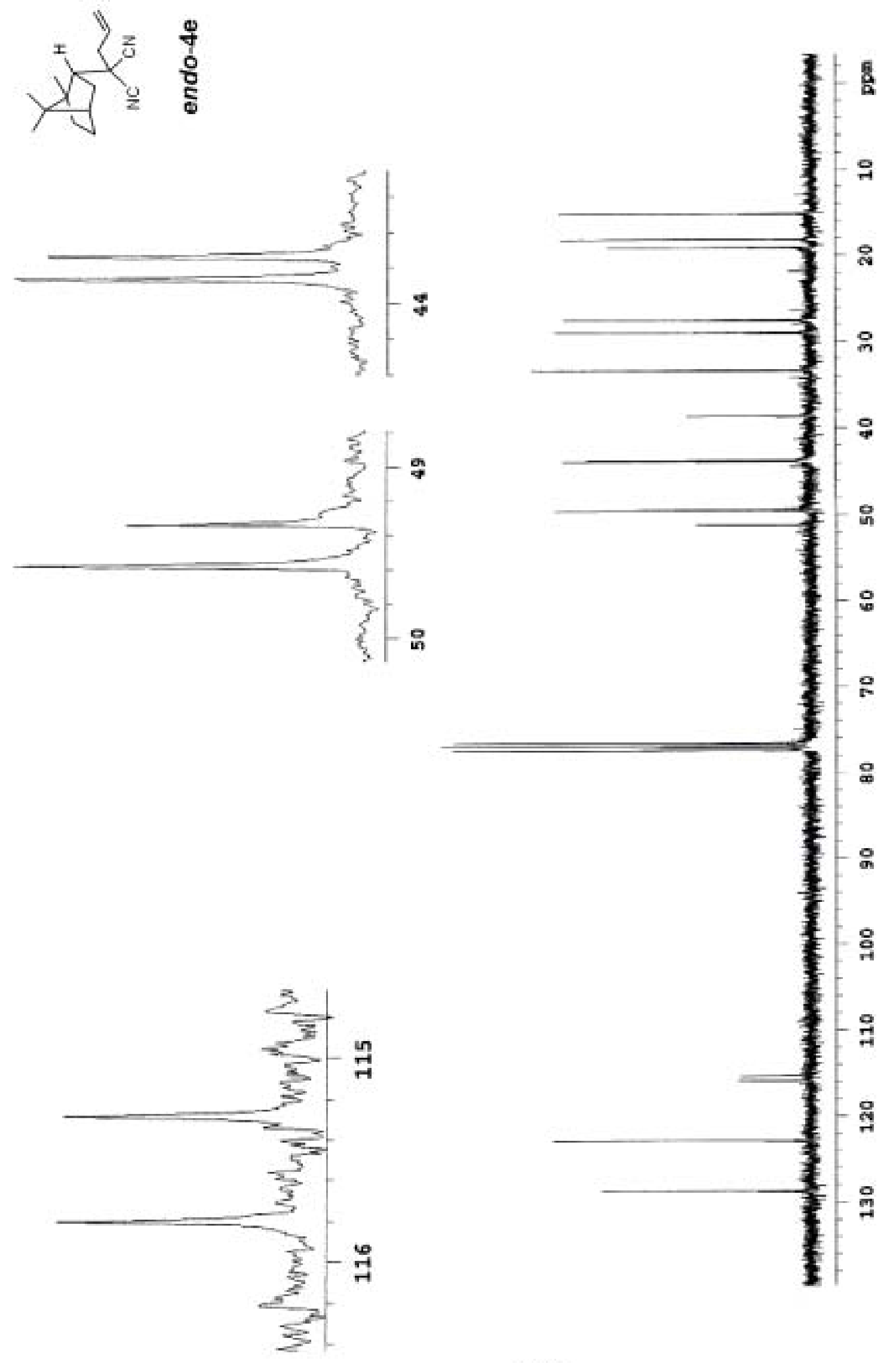
$\sqrt{\gamma_{0}^{\circ}}$

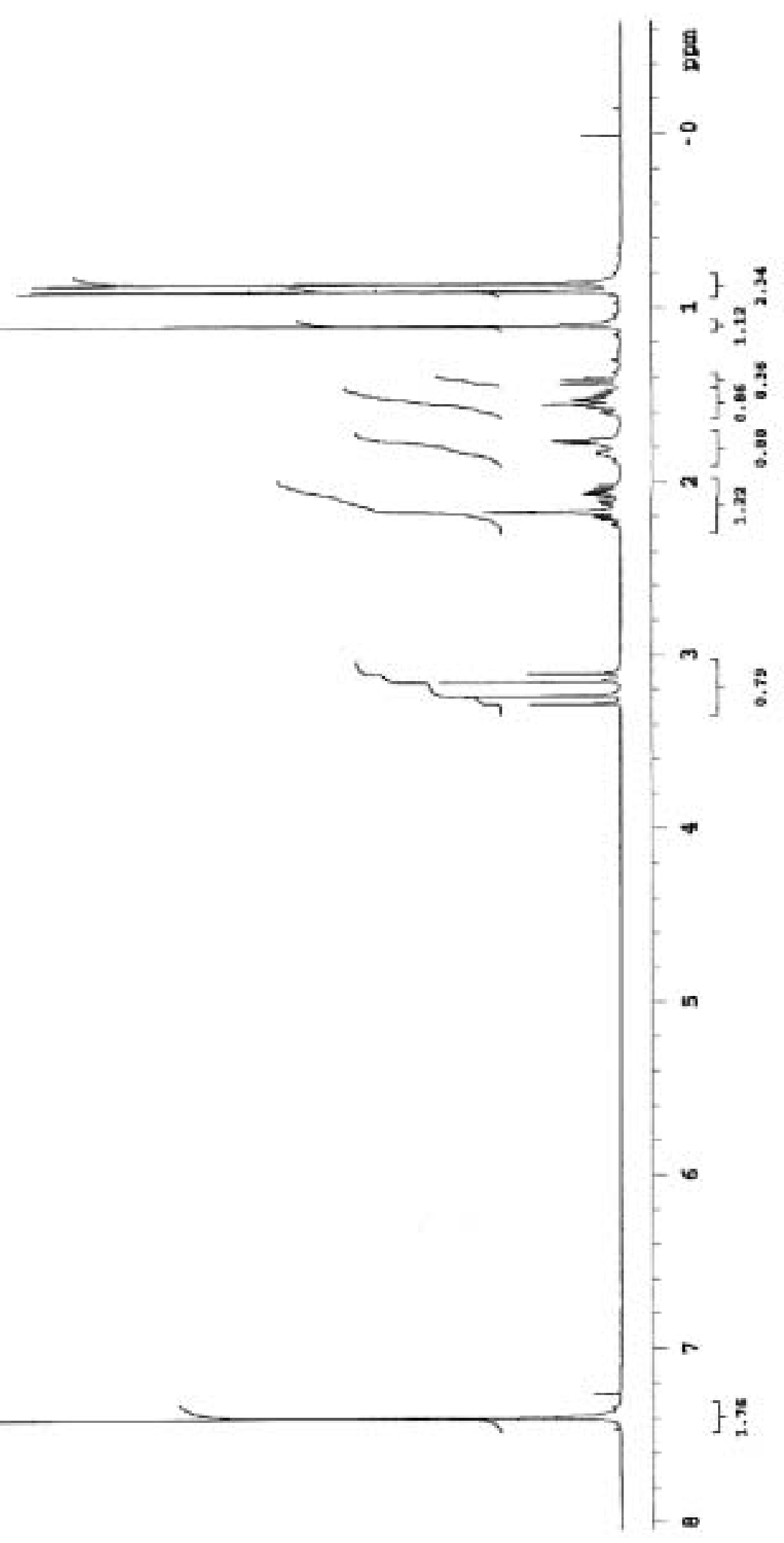



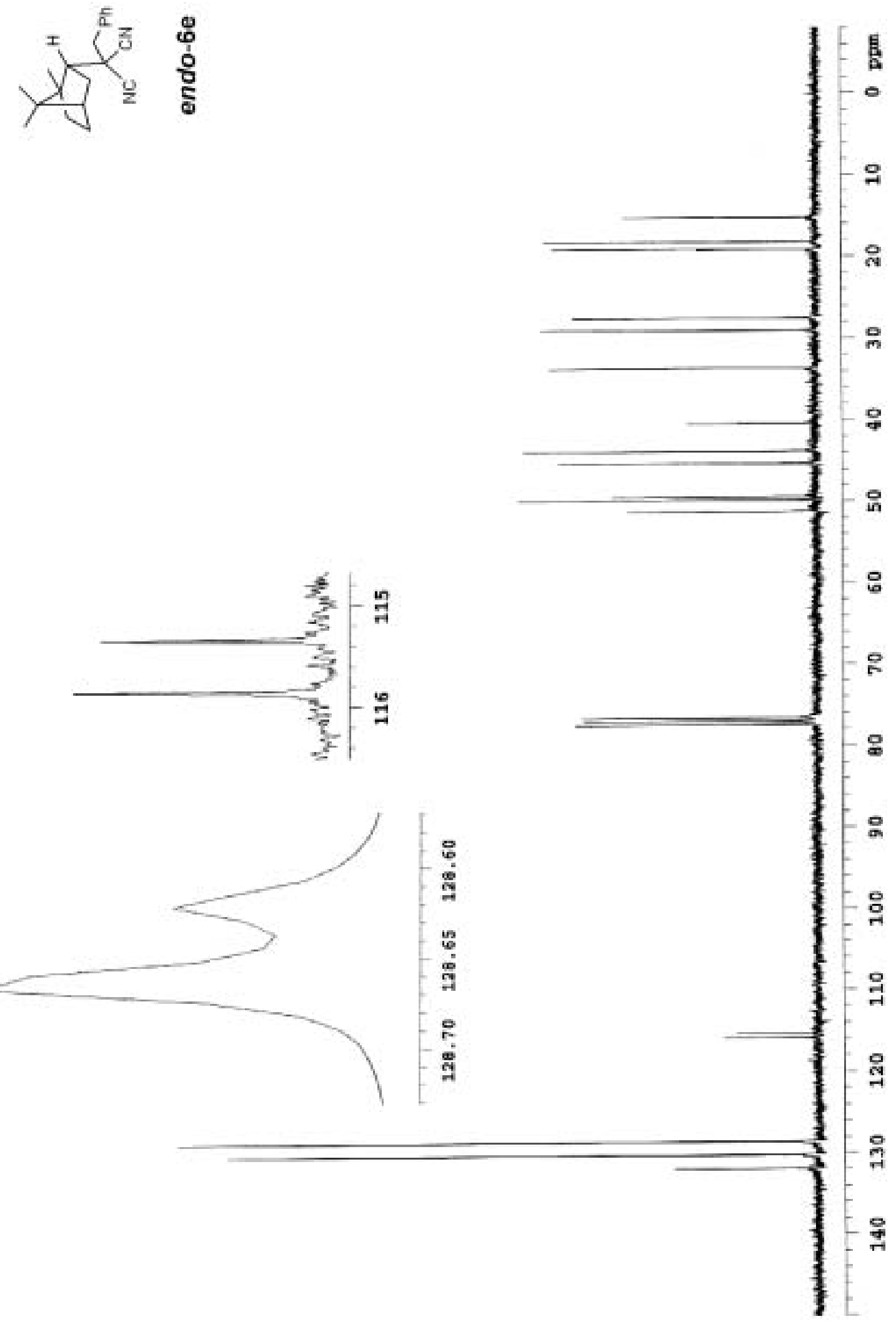

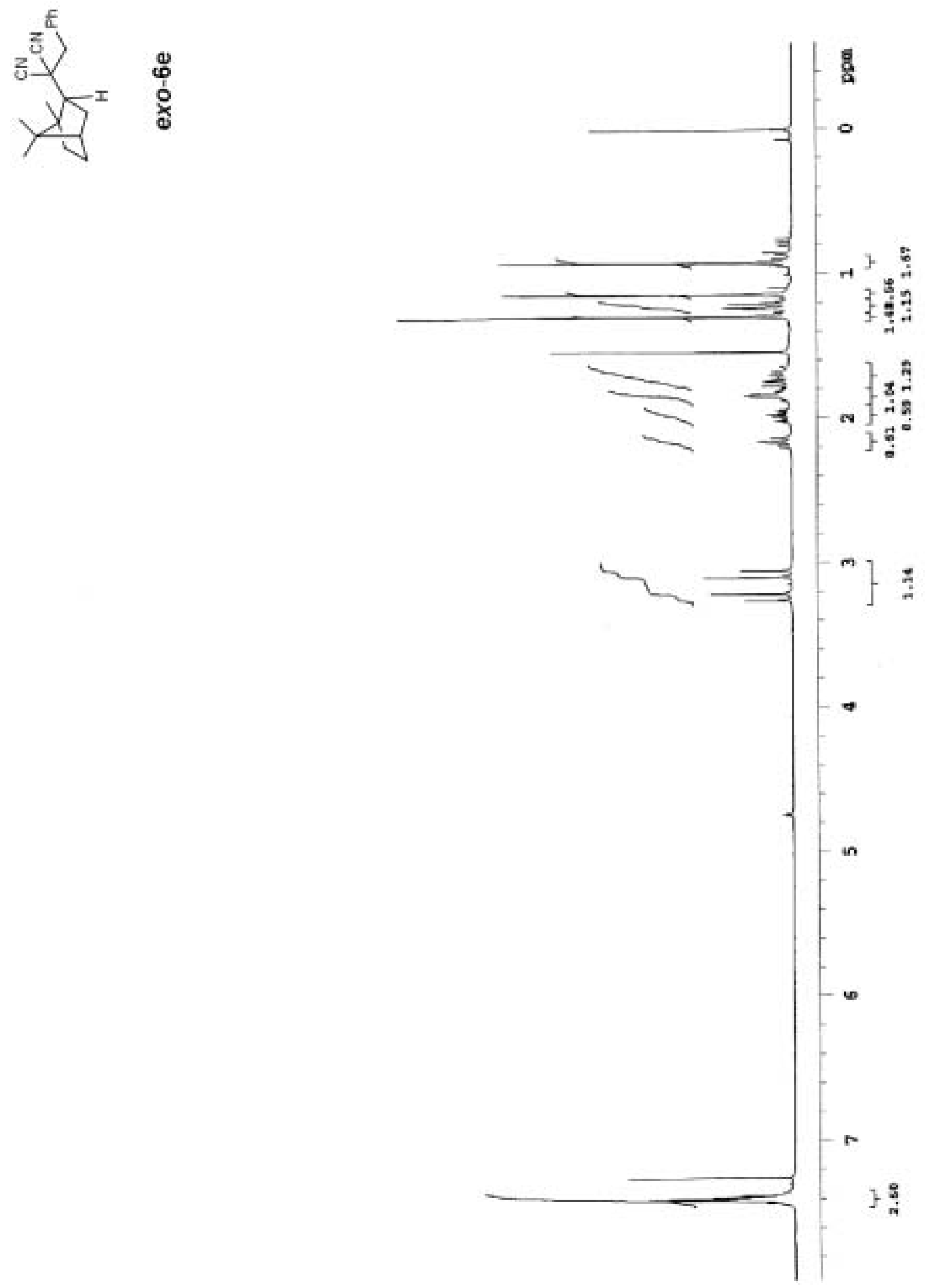


$$
1
$$




\section{X-ray crystallographic Data of cis-3a}
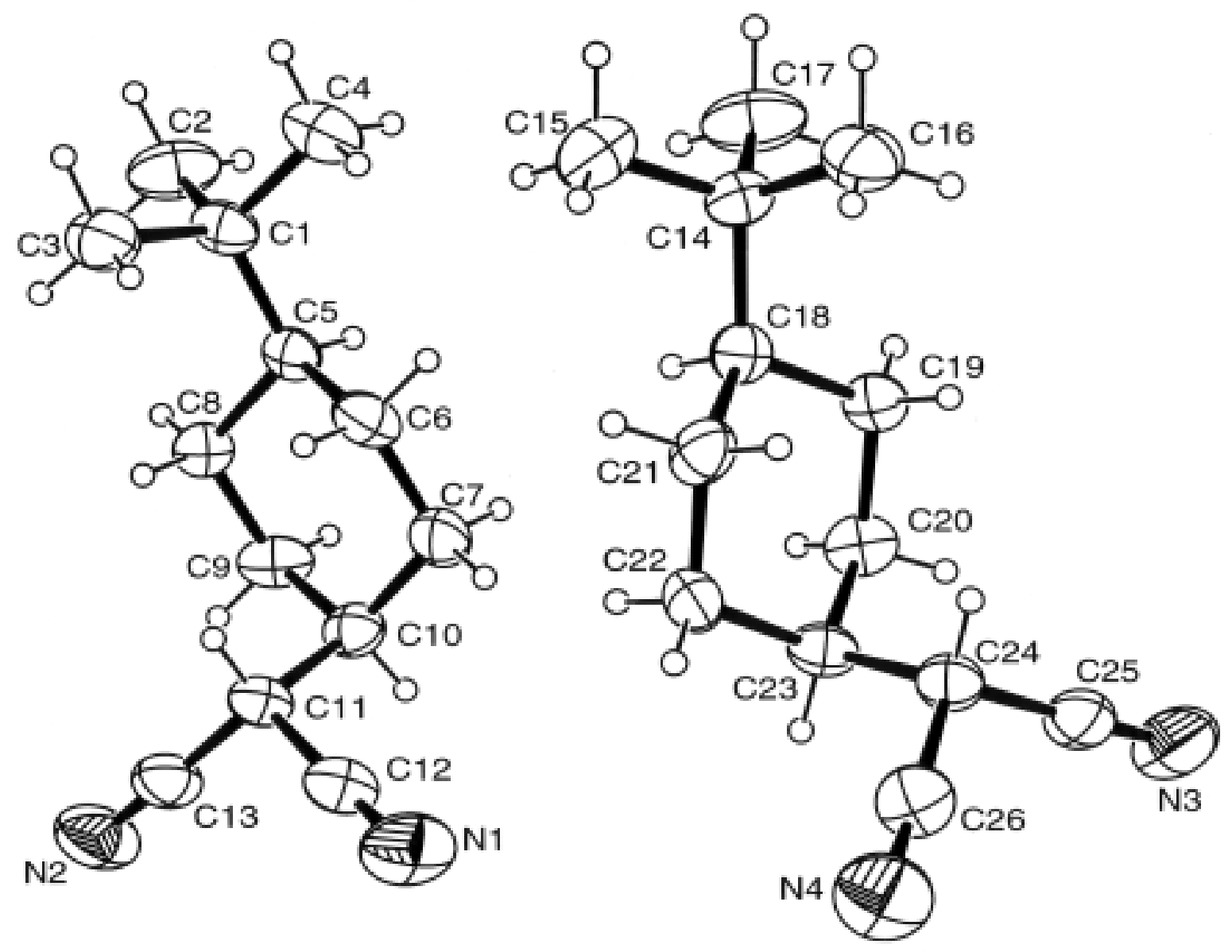

Figure S1. ORTEP drawing of $\boldsymbol{c i s - 3 a}$ (ellipsoids at 30\% probability). 
Experimental

\section{Data Collection}

A colorless prism crystal of $\mathrm{C}_{13} \mathrm{H}_{20} \mathrm{~N}_{2}$ having approximate dimensions of $0.80 \mathrm{x}$ $0.35 \times 0.10 \mathrm{~mm}$ was mounted on a glass fiber. All measurements were made on a Rigaku RAXIS RAPID imaging plate area detector with graphite monochromated Mo-Ka radiation.

Indexing was performed from 30 oscillations that were exposed for 180 seconds. The crystal-to-detector distance was $127.40 \mathrm{~mm}$.

Cell constants and an orientation matrix for data collection corresponded to a primitive triclinic cell with dimensions:

$$
\begin{array}{lrl}
\mathrm{a}= & 8.3392(3) \AA & \alpha=103.712(6)^{\mathrm{O}} \\
\mathrm{b}= & 11.6677(4) \AA & \beta=99.04(1)^{\mathrm{O}} \\
\mathrm{c}= & 15.175(2) \AA & \gamma=104.228(5)^{\mathrm{O}} \\
\mathrm{V}=1353.5(2) \AA^{3} &
\end{array}
$$

For $Z=4$ and F.W. $=204.31$, the calculated density is $1.00 \mathrm{~g} / \mathrm{cm}^{3}$. Based on a statistical analysis of intensity distribution, and the successful solution and refinement of the structure, the space group was determined to be:

$$
\text { P-1 (\#2) }
$$

The data were collected at a temperature of $23 \pm 1^{\circ} \mathrm{C}$ to a maximum $2 \theta$ value of $55.0^{\circ}$. A total of 44 oscillation images were collected. A sweep of data was done using $\omega$ scans from 130.0 to $190.0^{\circ}$ in $5.0^{\circ} \mathrm{step}$, at $\chi=45.0^{\circ}$ and $\phi=0.0^{\circ}$. The exposure rate was 300.0 [sec./0]. A second sweep was performed using $\omega$ scans from 0.0 to 160.00 in $5.0^{\circ}$ step, at $\chi=45.0^{\circ}$ and $\phi=180.0^{\circ}$. The exposure rate was 300.0 [sec. $/ 0$ ]. The crystal-to-detector distance was $127.40 \mathrm{~mm}$. Readout was performed in the $0.100 \mathrm{~mm}$ pixel mode.

\section{Data Reduction}


A total of 5113 reflections was collected.

The linear absorption coefficient, $\mu$, for Mo-K $\alpha$ radiation is $0.6 \mathrm{~cm}^{-1}$. An empirical absorption correction was applied which resulted in transmission factors ranging from 0.65 to 1.46 . The data were corrected for Lorentz and polarization effects.

\section{$\underline{\text { Structure Solution and Refinement }}$}

The structure was solved by direct methods 1 and expanded using Fourier techniques $^{2}$. The non-hydrogen atoms were refined anisotropically. Hydrogen atoms were refined using the riding model. The final cycle of full-matrix least-squares refinement ${ }^{3}$ on $F$ was based on 2515 observed reflections $(I>3.60 \sigma(I))$ and 311 variable parameters and converged (largest parameter shift was 0.00 times its esd) with unweighted and weighted agreement factors of:

$$
\begin{gathered}
\mathrm{R}=\Sigma|| \mathrm{Fo}|-| \mathrm{Fc} \| / \Sigma|\mathrm{Fo}|=0.100 \\
\mathrm{R}_{\mathrm{W}}=\left[\Sigma \mathrm{w}(|\mathrm{Fo}|-|\mathrm{Fc}|)^{2} / \Sigma \mathrm{w} \mathrm{Fo}{ }^{2}\right]^{1 / 2}=0.120
\end{gathered}
$$

The standard deviation of an observation of unit weight ${ }^{4}$ was 1.03. A Sheldrick weighting scheme was used. Plots of $\Sigma \mathrm{w}(|\mathrm{Fo}|-|\mathrm{Fc}|)^{2}$ versus $|\mathrm{Fo}|$, reflection order in data collection, $\sin \theta / \lambda$ and various classes of indices showed no unusual trends. The maximum and minimum peaks on the final difference Fourier map corresponded to 0.62 and $-0.55 \mathrm{e}^{-} / \AA^{3}$, respectively.

Neutral atom scattering factors were taken from Cromer and Waber ${ }^{5}$. Anomalous dispersion effects were included in Fcalc 6 ; the values for $\Delta f^{\prime}$ and $\Delta f^{\prime \prime}$ were those of Creagh and McAuley7. The values for the mass attenuation coefficients are those of Creagh and Hubbell 8 . All calculations were performed using the CrystalStructure 9,10 crystallographic software package.

References

(1) SIR92: Altomare, A., Cascarano, G., Giacovazzo, C., Guagliardi, A., Burla, M., 
Polidori, G., and Camalli, M. (1994) J. Appl. Cryst., 27, 435.

(2) DIRDIF99: Beurskens, P.T., Admiraal, G., Beurskens, G., Bosman, W.P., de Gelder, R., Israel, R. and Smits, J.M.M.(1999). The DIRDIF-99 program system, Technical Report of the Crystallography Laboratory, University of Nijmegen, The Netherlands.

(3) Least Squares function minimized:

$$
\Sigma w\left(\left|F_{0}\right|-\left|F_{c}\right|\right)^{2} \quad \text { where } w=\text { Least Squares weights. }
$$

(4) Standard deviation of an observation of unit weight:

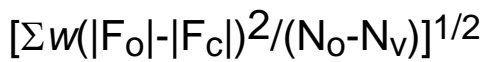

$$
\begin{aligned}
& \text { where: } \quad \mathrm{N}_{\mathrm{O}}=\text { number of observations } \\
& \mathrm{N}_{\mathrm{V}}=\text { number of variables }
\end{aligned}
$$

(5) Cromer, D. T. \& Waber, J. T.; "International Tables for X-ray Crystallography", Vol. IV, The Kynoch Press, Birmingham, England, Table 2.2 A (1974).

(6) Ibers, J. A. \& Hamilton, W. C.; Acta Crystallogr., 17, 781 (1964).

(7) Creagh, D. C. \& McAuley, W.J .; "International Tables for Crystallography", Vol C, (A.J.C. Wilson, ed.), Kluwer Academic Publishers, Boston, Table 4.2.6.8, pages 219-222 (1992).

(8) Creagh, D. C. \& Hubbell, J.H..; "International Tables for Crystallography", Vol C, (A.J.C. Wilson, ed.), Kluwer Academic Publishers, Boston, Table 4.2.4.3, pages 200-206 (1992).

(9) CrystalStructure 3.5.1: Crystal Structure Analysis Package, Rigaku and Rigaku/MSC (2000-2003). 9009 New Trails Dr. The Woodlands TX 77381 USA.

(10) CRYSTALS Issue 10: Watkin, D.J., Prout, C.K. Carruthers, J.R. \& Betteridge, P.W. Chemical Crystallography Laboratory, Oxford, UK. (1996) 
EXPERIMENTAL DETAILS

\author{
A. Crystal Data
}

Empirical Formula

Formula Weight

Crystal Color, Habit

Crystal Dimensions

Crystal System

Lattice Type

Indexing Images

Detector Position

Pixel Size

Lattice Parameters

Space Group

$Z$ value

$D_{\text {calc }}$
$\mathrm{C}_{13} \mathrm{H}_{20} \mathrm{~N}_{2}$

204.31

colorless, prism

$0.80 \times 0.35 \times 0.10 \mathrm{~mm}$

triclinic

Primitive

3 oscillations @ 180.0 seconds

$127.40 \mathrm{~mm}$

$0.100 \mathrm{~mm}$

$$
\begin{aligned}
& a=8.3392(3) \AA \\
& b=11.6677(4) \AA \\
& c=15.175(2) \AA \\
& \alpha=103.712(6) \circ \\
& \beta=99.04(1) \circ \\
& \gamma=104.228(5) \circ \\
& V=1353.5(2) \AA^{3}
\end{aligned}
$$

P-1 (\#2)

4

$1.003 \mathrm{~g} / \mathrm{cm}^{3}$ 
$F_{000}$

$\mu(\operatorname{MoK} \alpha)$
448.00

$0.59 \mathrm{~cm}^{-1}$ 
B. Intensity Measurements

Diffractometer

Radiation

Detector Aperture

Data Images

$\omega$ oscillation Range $(\chi=45.0, \phi=0.0)$

Exposure Rate

$\omega$ oscillation Range ( $\chi=45.0, \phi=180.0)$

Exposure Rate

Detector Position

Pixel Size

$2 \theta_{\max }$

No. of Reflections Measured

Corrections
Rigaku RAXIS-RAPID

$\operatorname{MoK} \alpha(\lambda=0.71069 \AA)$

graphite monochromated

$270 \mathrm{~mm} \times 256 \mathrm{~mm}$

44 exposures

$130.0-190.00$

$300.0 \mathrm{sec} . / 0$

$0.0-160.00$

$300.0 \mathrm{sec} . / 0$

$127.40 \mathrm{~mm}$

$0.100 \mathrm{~mm}$

$55.0^{0}$

Total: 5113

Lorentz-polarization

Absorption

(trans. factors: $0.6519-1.4612$ ) 
C. Structure Solution and Refinement

Structure Solution

Refinement

Function Minimized

Least Squares Weights

Anomalous Dispersion

No. Observations $(\mathrm{I}>3.60 \sigma(\mathrm{I}))$

No. Variables

Reflection/Parameter Ratio

Residuals: R (I>3.60\%(I))

Residuals: Rw $(I>3.60 \sigma(I))$

Goodness of Fit Indicator

Max Shift/Error in Final Cycle

Maximum peak in Final Diff. Map

Minimum peak in Final Diff. Map
Direct Methods (SIR92)

Full-matrix least-squares on $\mathrm{F}$

$\Sigma w(|\mathrm{Fo}|-|\mathrm{Fc}|)^{2}$

$1 /\left[0.0007 \mathrm{Fo}^{2}+1.0000 \sigma\left(\mathrm{Fo}^{2}\right)+0.0900\right]$

All non-hydrogen atoms

2515

311

8.09

0.100

0.120

1.033

0.000

$0.62 \mathrm{e}^{-/ \AA^{3}}$

$-0.55 \mathrm{e}^{-/ / \AA^{3}}$ 
Table 1. Atomic coordinates and $\mathrm{B}_{\text {iso }} / \mathrm{B}_{\text {eq }}$

$\begin{array}{lcccc}\text { atom } & x & y & z & B_{\text {eq }} \\ \mathrm{N}(1) & 0.5616 & 0.3098 & 0.0153 & 8.757 \\ \mathrm{~N}(2) & 0.3237 & 0.0026 & 0.1103 & 9.590 \\ \mathrm{~N}(3) & 0.8173 & 1.0047 & 0.1119 & 9.538 \\ \mathrm{~N}(4) & 0.8335 & 0.7048 & -0.1154 & 8.912 \\ \mathrm{C}(23) & 0.8777 & 0.7175 & 0.1201 & 4.974 \\ \mathrm{C}(5) & 0.9161 & 0.2992 & 0.3804 & 4.527 \\ \mathrm{C}(8) & 0.7535 & 0.1883 & 0.3496 & 4.717 \\ \mathrm{C}(26) & 0.8785 & 0.7540 & -0.0372 & 6.075 \\ \mathrm{C}(19) & 1.1147 & 0.8193 & 0.2710 & 4.630 \\ \mathrm{C}(21) & 1.1518 & 0.6517 & 0.1445 & 5.535 \\ \mathrm{C}(24) & 0.9337 & 0.8132 & 0.0662 & 4.749 \\ \mathrm{C}(14) & 1.3785 & 0.7378 & 0.3026 & 4.903 \\ \mathrm{C}(7) & 0.7606 & 0.3980 & 0.2721 & 6.172 \\ \mathrm{C}(1) & 1.0796 & 0.2683 & 0.4178 & 5.190 \\ \mathrm{C}(20) & 0.9218 & 0.7795 & 0.2267 & 5.616 \\ \mathrm{C}(25) & 0.8671 & 0.9208 & 0.0899 & 6.135 \\ \mathrm{C}(18) & 1.1894 & 0.7090 & 0.2509 & 4.586 \\ \mathrm{C}(11) & 0.5832 & 0.1980 & 0.1485 & 4.835 \\ \mathrm{C}(10) & 0.5982 & 0.2930 & 0.2452 & 5.198 \\ \mathrm{C}(22) & 0.9613 & 0.6139 & 0.0997 & 5.797 \\ \mathrm{C}(6) & 0.9207 & 0.3596 & 0.3002 & 5.672 \\ \mathrm{C}(12) & 0.5702 & 0.2599 & 0.0708 & 5.927 \\ \mathrm{C}(13) & 0.4352 & 0.0870 & 0.1251 & 6.356 \\ \mathrm{C}(9) & 0.5933 & 0.2269 & 0.3237 & 5.794 \\ \mathrm{C}(4) & 1.2366 & 0.3840 & 0.4451 & 7.554 \\ \mathrm{C}(17) & 1.3916 & 0.7738 & 0.4088 & 8.764 \\ \mathrm{C}(16) & 1.4948 & 0.8433 & 0.2765 & 7.627 \\ \mathrm{C}(3) & 1.1143 & 0.1627 & 0.3454 & 7.438 \\ \mathrm{C}(2) & 1.0668 & 0.2246 & 0.5073 & 8.642 \\ \mathrm{C}(15) & 1.4437 & 0.6239 & 0.2782 & 7.757 \\ \mathrm{H}(1) & 1.0500 & 0.2886 & 0.5535 & 10.718 \\ \mathrm{H}(2) & 0.9728 & 0.1525 & 0.4924 & 10.718 \\ \mathrm{H}(3) & 1.1680 & 0.2070 & 0.5304 & 10.720 \\ \mathrm{H}(4) & 1.0228 & 0.0893 & 0.3313 & 8.440 \\ \mathrm{H}(5) & 1.1241 & 0.1857 & 0.2900 & 8.436 \\ \mathrm{H}(6) & 1.2169 & 0.1487 & 0.3708 & 8.434 \\ \mathrm{H}(7) & 1.3363 & 0.3653 & 0.4690 & 8.178\end{array}$


Table 1. Atomic coordinates and $\mathrm{B}_{\mathrm{iso}} / \mathrm{B}_{\mathrm{eq}}$ (continued)

$\begin{array}{lcccc}\text { atom } & x & y & z & B_{\text {eq }} \\ \mathrm{H}(8) & 1.2497 & 0.4114 & 0.3918 & 8.176 \\ \mathrm{H}(9) & 1.2183 & 0.4471 & 0.4914 & 8.181 \\ \mathrm{H}(10) & 0.8987 & 0.3573 & 0.4307 & 5.261 \\ \mathrm{H}(11) & 0.7638 & 0.1317 & 0.2959 & 5.822 \\ \mathrm{H}(12) & 0.7441 & 0.1497 & 0.3978 & 5.834 \\ \mathrm{H}(13) & 0.4977 & 0.1553 & 0.3023 & 7.037 \\ \mathrm{H}(14) & 0.5818 & 0.2806 & 0.3786 & 7.041 \\ \mathrm{H}(15) & 1.0188 & 0.4293 & 0.3180 & 6.262 \\ \mathrm{H}(16) & 0.9284 & 0.3004 & 0.2472 & 6.260 \\ \mathrm{H}(17) & 0.7707 & 0.4325 & 0.2218 & 7.000 \\ \mathrm{H}(18) & 0.7567 & 0.4592 & 0.3249 & 7.006 \\ \mathrm{H}(19) & 0.5043 & 0.3254 & 0.2388 & 6.323 \\ \mathrm{H}(20) & 0.6840 & 0.1735 & 0.1530 & 5.526 \\ \mathrm{H}(21) & 1.5060 & 0.7934 & 0.4419 & 10.516 \\ \mathrm{H}(22) & 1.3514 & 0.8435 & 0.4254 & 10.515 \\ \mathrm{H}(23) & 1.3231 & 0.7066 & 0.4240 & 10.517 \\ \mathrm{H}(24) & 1.3745 & 0.5582 & 0.2947 & 9.757 \\ \mathrm{H}(25) & 1.4389 & 0.5993 & 0.2133 & 9.756 \\ \mathrm{H}(26) & 1.5578 & 0.6442 & 0.3120 & 9.751 \\ \mathrm{H}(27) & 1.6079 & 0.8620 & 0.3112 & 8.977 \\ \mathrm{H}(28) & 1.4918 & 0.8192 & 0.2118 & 8.973 \\ \mathrm{H}(29) & 1.4563 & 0.9142 & 0.2911 & 8.976 \\ \mathrm{H}(30) & 1.1239 & 0.6483 & 0.2735 & 5.641 \\ \mathrm{H}(31) & 1.1731 & 0.8792 & 0.2450 & 5.517 \\ \mathrm{H}(32) & 1.1337 & 0.8538 & 0.3364 & 5.520 \\ \mathrm{H}(33) & 0.8845 & 0.8512 & 0.2362 & 6.963 \\ \mathrm{H}(34) & 0.8638 & 0.7249 & 0.2567 & 6.967 \\ \mathrm{H}(35) & 1.2102 & 0.7124 & 0.1193 & 6.848 \\ \mathrm{H}(36) & 1.1935 & 0.5821 & 0.1319 & 6.838 \\ \mathrm{H}(37) & 0.9068 & 0.5491 & 0.1225 & 6.497 \\ \mathrm{H}(38) & 0.9458 & 0.5843 & 0.0340 & 6.496 \\ \mathrm{H}(39) & 0.7581 & 0.6823 & 0.0992 & 5.785 \\ \mathrm{H}(40) & 1.0543 & 0.8431 & 0.0831 & 5.547\end{array}$

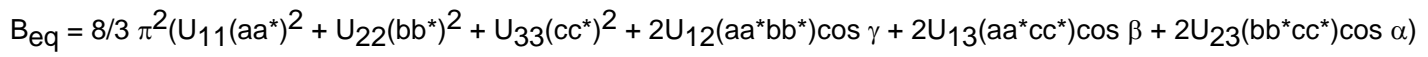


Table 2. Anisotropic Displacement Parameters

\begin{tabular}{lcccccc} 
atom & $\mathrm{U}_{11}$ & $\mathrm{U}_{22}$ & $\mathrm{U}_{33}$ & $\mathrm{U}_{12}$ & \multicolumn{1}{c}{$\mathrm{U}_{13}$} & $\mathrm{U}_{23}$ \\
$\mathrm{~N}(1)$ & 0.1155 & 0.1482 & 0.0733 & 0.0210 & 0.0211 & 0.0586 \\
$\mathrm{~N}(2)$ & 0.1176 & 0.1083 & 0.1031 & -0.0211 & -0.0135 & 0.0467 \\
$\mathrm{~N}(3)$ & 0.1712 & 0.1132 & 0.0738 & 0.0864 & -0.0102 & -0.0021 \\
$\mathrm{~N}(4)$ & 0.1458 & 0.1341 & 0.0578 & 0.0445 & 0.0313 & 0.0176 \\
$\mathrm{C}(23)$ & 0.0433 & 0.0806 & 0.0595 & 0.0125 & 0.0094 & 0.0173 \\
$\mathrm{C}(5)$ & 0.0585 & 0.0639 & 0.0440 & 0.0178 & 0.0141 & 0.0039 \\
$\mathrm{C}(8)$ & 0.0600 & 0.0727 & 0.0518 & 0.0165 & 0.0197 & 0.0263 \\
$\mathrm{C}(26)$ & 0.0841 & 0.0897 & 0.0621 & 0.0303 & 0.0251 & 0.0206 \\
$\mathrm{C}(19)$ & 0.0677 & 0.0693 & 0.0378 & 0.0227 & 0.0156 & 0.0085 \\
$\mathrm{C}(21)$ & 0.0883 & 0.0707 & 0.0577 & 0.0414 & 0.0139 & 0.0135 \\
$\mathrm{C}(24)$ & 0.0641 & 0.0668 & 0.0451 & 0.0179 & 0.0085 & 0.0117 \\
$\mathrm{C}(14)$ & 0.0568 & 0.0775 & 0.0568 & 0.0235 & 0.0111 & 0.0256 \\
$\mathrm{C}(7)$ & 0.0954 & 0.0671 & 0.0593 & 0.0169 & -0.0002 & 0.0136 \\
$\mathrm{C}(1)$ & 0.0615 & 0.0802 & 0.0452 & 0.0150 & 0.0116 & 0.0056 \\
$\mathrm{C}(20)$ & 0.0646 & 0.0945 & 0.0616 & 0.0284 & 0.0241 & 0.0245 \\
$\mathrm{C}(25)$ & 0.0961 & 0.0768 & 0.0494 & 0.0331 & -0.0033 & 0.0031 \\
$\mathrm{C}(18)$ & 0.0601 & 0.0630 & 0.0555 & 0.0142 & 0.0185 & 0.0256 \\
$\mathrm{C}(11)$ & 0.0556 & 0.0717 & 0.0480 & 0.0101 & 0.0053 & 0.0150 \\
$\mathrm{C}(10)$ & 0.0585 & 0.0775 & 0.0643 & 0.0292 & 0.0145 & 0.0158 \\
$\mathrm{C}(22)$ & 0.0860 & 0.0528 & 0.0668 & 0.0097 & -0.0006 & 0.0131 \\
$\mathrm{C}(6)$ & 0.0656 & 0.0724 & 0.0601 & -0.0045 & 0.0040 & 0.0195 \\
$\mathrm{C}(12)$ & 0.0658 & 0.0904 & 0.0564 & 0.0107 & 0.0071 & 0.0152 \\
$\mathrm{C}(13)$ & 0.0825 & 0.0858 & 0.0610 & 0.0084 & -0.0028 & 0.0280 \\
$\mathrm{C}(9)$ & 0.0587 & 0.1162 & 0.0480 & 0.0270 & 0.0177 & 0.0239 \\
$\mathrm{C}(4)$ & 0.0718 & 0.1019 & 0.0853 & 0.0060 & 0.0047 & 0.0040 \\
$\mathrm{C}(17)$ & 0.0906 & 0.1851 & 0.0572 & 0.0513 & 0.0066 & 0.0305 \\
$\mathrm{C}(16)$ & 0.0570 & 0.1040 & 0.1231 & 0.0097 & 0.0108 & 0.0433 \\
$\mathrm{C}(3)$ & 0.0705 & 0.1034 & 0.0932 & 0.0405 & 0.0077 & -0.0085 \\
$\mathrm{C}(2)$ & 0.0871 & 0.1742 & 0.0780 & 0.0399 & 0.0112 & 0.0603 \\
$\mathrm{C}(15)$ & 0.0922 & 0.1091 & 0.1075 & 0.0475 & 0.0156 & 0.0437
\end{tabular}

The general temperature factor expression: $\exp \left(-2 \pi^{2}\left(a^{*} U_{11} h^{2}+b^{* 2} U_{22} k^{2}+\left.c^{\star 2} U_{33}\right|^{2}\right.\right.$ $\left.\left.+2 a^{*} b^{*} U_{12} h k+2 a^{*} c^{*} U_{13} h l+2 b^{*} c^{*} U_{23} k l\right)\right)$ 
Table 3. Bond lengths $(\AA ̊)$

$\begin{array}{llllll}\text { atom } & \text { atom } & \text { distance } & \text { atom } & \text { atom } & \text { distance } \\ \mathrm{N}(1) & \mathrm{C}(12) & 1.135612 & \mathrm{~N}(2) & \mathrm{C}(13) & 1.124032 \\ \mathrm{~N}(3) & \mathrm{C}(25) & 1.153742 & \mathrm{~N}(4) & \mathrm{C}(26) & 1.145458 \\ \mathrm{C}(23) & \mathrm{C}(24) & 1.559565 & \mathrm{C}(23) & \mathrm{C}(20) & 1.549631 \\ \mathrm{C}(23) & \mathrm{C}(22) & 1.531615 & \mathrm{C}(5) & \mathrm{C}(8) & 1.543382 \\ \mathrm{C}(5) & \mathrm{C}(1) & 1.551496 & \mathrm{C}(5) & \mathrm{C}(6) & 1.544621 \\ \mathrm{C}(8) & \mathrm{C}(9) & 1.535208 & \mathrm{C}(26) & \mathrm{C}(24) & 1.499940 \\ \mathrm{C}(19) & \mathrm{C}(20) & 1.549188 & \mathrm{C}(19) & \mathrm{C}(18) & 1.551109 \\ \mathrm{C}(21) & \mathrm{C}(18) & 1.542192 & \mathrm{C}(21) & \mathrm{C}(22) & 1.534093 \\ \mathrm{C}(24) & \mathrm{C}(25) & 1.485354 & \mathrm{C}(14) & \mathrm{C}(18) & 1.562033 \\ \mathrm{C}(14) & \mathrm{C}(17) & 1.543313 & \mathrm{C}(14) & \mathrm{C}(16) & 1.542067 \\ \mathrm{C}(14) & \mathrm{C}(15) & 1.543671 & \mathrm{C}(7) & \mathrm{C}(10) & 1.508343 \\ \mathrm{C}(7) & \mathrm{C}(6) & 1.539687 & \mathrm{C}(1) & \mathrm{C}(4) & 1.550106 \\ \mathrm{C}(1) & \mathrm{C}(3) & 1.562668 & \mathrm{C}(1) & \mathrm{C}(2) & 1.569718 \\ \mathrm{C}(11) & \mathrm{C}(10) & 1.581781 & \mathrm{C}(11) & \mathrm{C}(12) & 1.524333 \\ \mathrm{C}(11) & \mathrm{C}(13) & 1.478797 & \mathrm{C}(10) & \mathrm{C}(9) & 1.564732\end{array}$


Table 4. Bond lengths involving hydrogens $(\AA)$

$\begin{array}{llllll}\text { atom } & \text { atom } & \text { distance } & \text { atom } & \text { atom } & \text { distance } \\ \mathrm{C}(23) & \mathrm{H}(39) & 0.949986 & \mathrm{C}(5) & \mathrm{H}(10) & 0.949955 \\ \mathrm{C}(8) & \mathrm{H}(11) & 0.950041 & \mathrm{C}(8) & \mathrm{H}(12) & 0.949964 \\ \mathrm{C}(19) & \mathrm{H}(31) & 0.949994 & \mathrm{C}(19) & \mathrm{H}(32) & 0.950029 \\ \mathrm{C}(21) & \mathrm{H}(35) & 0.950001 & \mathrm{C}(21) & \mathrm{H}(36) & 0.950016 \\ \mathrm{C}(24) & \mathrm{H}(40) & 0.949968 & \mathrm{C}(7) & \mathrm{H}(17) & 0.950016 \\ \mathrm{C}(7) & \mathrm{H}(18) & 0.950033 & \mathrm{C}(20) & \mathrm{H}(33) & 0.950023 \\ \mathrm{C}(20) & \mathrm{H}(34) & 0.950025 & \mathrm{C}(18) & \mathrm{H}(30) & 0.950031 \\ \mathrm{C}(11) & \mathrm{H}(20) & 0.949970 & \mathrm{C}(10) & \mathrm{H}(19) & 0.949968 \\ \mathrm{C}(22) & \mathrm{H}(37) & 0.949939 & \mathrm{C}(22) & \mathrm{H}(38) & 0.950010 \\ \mathrm{C}(6) & \mathrm{H}(15) & 0.949975 & \mathrm{C}(6) & \mathrm{H}(16) & 0.950101 \\ \mathrm{C}(9) & \mathrm{H}(13) & 0.950040 & \mathrm{C}(9) & \mathrm{H}(14) & 0.949989 \\ \mathrm{C}(4) & \mathrm{H}(7) & 0.949951 & \mathrm{C}(4) & \mathrm{H}(8) & 0.949950 \\ \mathrm{C}(4) & \mathrm{H}(9) & 0.950048 & \mathrm{C}(17) & \mathrm{H}(21) & 0.949982 \\ \mathrm{C}(17) & \mathrm{H}(22) & 0.950032 & \mathrm{C}(17) & \mathrm{H}(23) & 0.949985 \\ \mathrm{C}(16) & \mathrm{H}(27) & 0.949967 & \mathrm{C}(16) & \mathrm{H}(28) & 0.950045 \\ \mathrm{C}(16) & \mathrm{H}(29) & 0.949960 & \mathrm{C}(3) & \mathrm{H}(4) & 0.949953 \\ \mathrm{C}(3) & \mathrm{H}(5) & 0.950089 & \mathrm{C}(3) & \mathrm{H}(6) & 0.949978 \\ \mathrm{C}(2) & \mathrm{H}(1) & 0.950106 & \mathrm{C}(2) & \mathrm{H}(2) & 0.950012 \\ \mathrm{C}(2) & \mathrm{H}(3) & 0.950034 & \mathrm{C}(15) & \mathrm{H}(24) & 0.950060 \\ \mathrm{C}(15) & \mathrm{H}(25) & 0.950099 & \mathrm{C}(15) & \mathrm{H}(26) & 0.949922\end{array}$


Table 5. Bond angles (0)

$\begin{array}{llllllll}\text { atom } & \text { atom } & \text { atom } & \text { angle } & \text { atom } & \text { atom } & \text { atom } & \text { angle } \\ \mathrm{C}(24) & \mathrm{C}(23) & \mathrm{C}(20) & 111.548612 & \mathrm{C}(24) & \mathrm{C}(23) & \mathrm{C}(22) & 111.176156 \\ \mathrm{C}(20) & \mathrm{C}(23) & \mathrm{C}(22) & 109.100356 & \mathrm{C}(8) & \mathrm{C}(5) & \mathrm{C}(1) & 114.127378 \\ \mathrm{C}(8) & \mathrm{C}(5) & \mathrm{C}(6) & 107.054541 & \mathrm{C}(1) & \mathrm{C}(5) & \mathrm{C}(6) & 116.443739 \\ \mathrm{C}(9) & \mathrm{C}(8) & \mathrm{C}(5) & 111.938187 & \mathrm{C}(24) & \mathrm{C}(26) & \mathrm{N}(4) & 177.523881 \\ \mathrm{C}(20) & \mathrm{C}(19) & \mathrm{C}(18) & 111.666563 & \mathrm{C}(18) & \mathrm{C}(21) & \mathrm{C}(22) & 111.920304 \\ \mathrm{C}(25) & \mathrm{C}(24) & \mathrm{C}(23) & 113.055946 & \mathrm{C}(25) & \mathrm{C}(24) & \mathrm{C}(26) & 108.663507 \\ \mathrm{C}(23) & \mathrm{C}(24) & \mathrm{C}(26) & 111.011499 & \mathrm{C}(18) & \mathrm{C}(14) & \mathrm{C}(17) & 109.079223 \\ \mathrm{C}(18) & \mathrm{C}(14) & \mathrm{C}(16) & 110.766880 & \mathrm{C}(18) & \mathrm{C}(14) & \mathrm{C}(15) & 111.724846 \\ \mathrm{C}(17) & \mathrm{C}(14) & \mathrm{C}(16) & 110.065845 & \mathrm{C}(17) & \mathrm{C}(14) & \mathrm{C}(15) & 107.152143 \\ \mathrm{C}(16) & \mathrm{C}(14) & \mathrm{C}(15) & 107.980804 & \mathrm{C}(10) & \mathrm{C}(7) & \mathrm{C}(6) & 113.458017 \\ \mathrm{C}(4) & \mathrm{C}(1) & \mathrm{C}(3) & 108.284773 & \mathrm{C}(4) & \mathrm{C}(1) & \mathrm{C}(2) & 107.279338 \\ \mathrm{C}(4) & \mathrm{C}(1) & \mathrm{C}(5) & 110.552452 & \mathrm{C}(3) & \mathrm{C}(1) & \mathrm{C}(2) & 106.787828 \\ \mathrm{C}(3) & \mathrm{C}(1) & \mathrm{C}(5) & 112.673881 & \mathrm{C}(2) & \mathrm{C}(1) & \mathrm{C}(5) & 111.031369 \\ \mathrm{C}(23) & \mathrm{C}(20) & \mathrm{C}(19) & 113.178138 & \mathrm{~N}(3) & \mathrm{C}(25) & \mathrm{C}(24) & 177.316983 \\ \mathrm{C}(19) & \mathrm{C}(18) & \mathrm{C}(21) & 108.592116 & \mathrm{C}(19) & \mathrm{C}(18) & \mathrm{C}(14) & 114.942192 \\ \mathrm{C}(21) & \mathrm{C}(18) & \mathrm{C}(14) & 114.809396 & \mathrm{C}(10) & \mathrm{C}(11) & \mathrm{C}(12) & 110.099502 \\ \mathrm{C}(10) & \mathrm{C}(11) & \mathrm{C}(13) & 112.598201 & \mathrm{C}(12) & \mathrm{C}(11) & \mathrm{C}(13) & 109.036989 \\ \mathrm{C}(9) & \mathrm{C}(10) & \mathrm{C}(7) & 108.937648 & \mathrm{C}(9) & \mathrm{C}(10) & \mathrm{C}(11) & 110.423015 \\ \mathrm{C}(7) & \mathrm{C}(10) & \mathrm{C}(11) & 111.246069 & \mathrm{C}(23) & \mathrm{C}(22) & \mathrm{C}(21) & 114.930665 \\ \mathrm{C}(5) & \mathrm{C}(6) & \mathrm{C}(7) & 113.743270 & \mathrm{~N}(1) & \mathrm{C}(12) & \mathrm{C}(11) & 177.648182 \\ \mathrm{~N}(2) & \mathrm{C}(13) & \mathrm{C}(11) & 177.742555 & \mathrm{C}(8) & \mathrm{C}(9) & \mathrm{C}(10) & 113.601510 \\ & & & & & & & \end{array}$


Table 6. Bond angles involving hydrogens ( $\left.{ }^{\circ}\right)$

\begin{tabular}{|c|c|c|c|c|c|c|c|}
\hline tom & atom & atom & angle & atom & atom & atom & angle \\
\hline$C(24)$ & $C(23)$ & $H(39)$ & 107.480101 & $C(20)$ & $C(23)$ & $H(39)$ & 108.949926 \\
\hline$C(22)$ & C(23) & $\mathrm{H}(39)$ & 108.504083 & $C(8)$ & $C(5)$ & $H(10)$ & 5.652663 \\
\hline$C(1)$ & $C(5)$ & $\mathrm{H}(10)$ & 106.793824 & $C(6)$ & C(5) & $H(10)$ & 105.946211 \\
\hline C(9) & $C(8)$ & $H(11)$ & 108.346337 & $C(9)$ & $C(8)$ & $H(12)$ & $109.8942 \varepsilon$ \\
\hline $\mathrm{H}(11)$ & $C(8)$ & $H(12)$ & 109.466153 & $H(11)$ & $C(8)$ & C(5) & \\
\hline$H(12)$ & C(8) & $C(5)$ & 109.828857 & $C(20)$ & $C(19)$ & $H(31)$ & .041870 \\
\hline$C(20)$ & $C(19)$ & $\mathrm{H}(32)$ & 109.493892 & $C(18)$ & $C(19)$ & $H(31)$ & 107.19404 \\
\hline$C(18)$ & $C(19)$ & $\mathrm{H}(32)$ & 109.441698 & $H(31)$ & $C(19)$ & $H(32)$ & 109.457883 \\
\hline$C(18)$ & $C(21)$ & $H(35)$ & 107.494533 & $C(18)$ & $C(21)$ & $H(36)$ & 108.919472 \\
\hline$C(22)$ & $C(21)$ & $\mathrm{H}(35)$ & 108.653687 & C(22) & $C(21)$ & $H(36)$ & 110.3232 \\
\hline$H(35)$ & $C(21)$ & $H(36)$ & 109.464471 & $C(25)$ & C(24) & $\mathrm{H}(40)$ & 107.75730 \\
\hline $\mathrm{H}(40)$ & $C(24)$ & $C(23)$ & 107.426258 & $\mathrm{H}(40)$ & $C(24)$ & $C(26)$ & 108.79403 \\
\hline$C(10)$ & $\mathrm{C}(7)$ & $\mathrm{H}(17)$ & 109.405302 & $C(10)$ & $\mathrm{C}(7)$ & $H(18)$ & 108.69992 \\
\hline$C(6)$ & $C(7)$ & $\mathrm{H}(17)$ & 109.291641 & $C(6)$ & $C(7)$ & $H(18)$ & 6435 \\
\hline $\mathrm{H}(17)$ & $C(7)$ & $\mathrm{H}(18)$ & 109.460171 & $H(33)$ & $C(20)$ & $H(34)$ & 109.4564 \\
\hline $\mathrm{H}(33)$ & $C(20)$ & $C(23)$ & 106.964347 & $H(33)$ & $C(20)$ & C(19) & 108.30259 \\
\hline $\mathrm{H}(34)$ & $C(20)$ & $C(23)$ & 110.090024 & $\mathrm{H}(34)$ & $C(20)$ & C(19) & 108.7763 \\
\hline$H(30)$ & $C(18)$ & $C(19)$ & 105.579067 & $H(30)$ & $C(18)$ & $C(21)$ & 105.5030 \\
\hline $\mathrm{H}(30)$ & $C(18)$ & $C(14)$ & 106.550932 & $C(10)$ & $C(11)$ & $\mathrm{H}(20)$ & 107.278131 \\
\hline$C(12)$ & $C(11)$ & $\mathrm{H}(20)$ & 108.954832 & $C(13)$ & $C(11)$ & $H(20)$ & 108.79518 \\
\hline$C(9)$ & $C(10)$ & $H(19)$ & 109.597178 & $H(19)$ & $C(10)$ & $C(7)$ & 108.93566 \\
\hline$H(19)$ & $C(10)$ & $C(11)$ & 107.670924 & $\mathrm{H}(37)$ & $C(22)$ & $H(38)$ & 9.46060 \\
\hline $\mathrm{H}(37)$ & $C(22)$ & $C(23)$ & 108.753678 & $\mathrm{H}(37)$ & $C(22)$ & $C(21)$ & 7.06595 \\
\hline $\mathrm{H}(38)$ & $C(22)$ & $C(23)$ & 108.013475 & $H(38)$ & $C(22)$ & $C(21)$ & 108.534 \\
\hline$H(15)$ & $C(6)$ & $H(16)$ & 109.451989 & $H(15)$ & $C(6)$ & C(5) & 108.67503 \\
\hline$H(15)$ & $C(6)$ & $\mathrm{C}(7)$ & 110.041960 & $H(16)$ & $C(6)$ & $C(5)$ & 107.55180 \\
\hline$H(16)$ & $C(6)$ & $C(7)$ & 107.281773 & $H(13)$ & $C(9)$ & $H(14)$ & 109.4584 \\
\hline$H(13)$ & $C(9)$ & $C(8)$ & 108.805980 & $H(13)$ & $C(9)$ & $C(10)$ & 107.799 \\
\hline$H(14)$ & $C(9)$ & $C(8)$ & 107.461020 & $H(14)$ & $C(9)$ & $C(10)$ & 109.66578 \\
\hline $\mathrm{H}(7)$ & $C(4)$ & $H(8)$ & 109.477038 & $\mathrm{H}(7)$ & $C(4)$ & $H(9)$ & 109.47425 \\
\hline $\mathrm{H}(7)$ & $\mathrm{C}(4)$ & $C(1)$ & 110.504738 & $H(8)$ & $C(4)$ & $\mathrm{H}(9)$ & 109.46924 \\
\hline $\mathrm{H}(8)$ & C(4) & C(1) & 109.367591 & $\mathrm{H}(9)$ & $\mathrm{C}(4)$ & $C(1)$ & 108.52830 \\
\hline $\mathrm{H}(21)$ & $C(17)$ & $\mathrm{H}(22)$ & 109.473603 & $\mathrm{H}(21)$ & C(17) & $\mathrm{H}(23)$ & 109.47075 \\
\hline $\mathrm{H}(21)$ & $C(17)$ & $C(14)$ & 110.673986 & $\mathrm{H}(22)$ & $C(17)$ & $H(23)$ & 109.47266 \\
\hline $\mathrm{H}(22)$ & $C(17)$ & $C(14)$ & 108.698913 & $\mathrm{H}(23)$ & $C(17)$ & $C(14)$ & 109.03060 \\
\hline $\mathrm{H}(27)$ & $C(16)$ & $\mathrm{H}(28)$ & 109.466272 & $\mathrm{H}(27)$ & $C(16)$ & $H(29)$ & 109.469312 \\
\hline $\mathrm{H}(27)$ & $C(16)$ & $C(14)$ & 109.309677 & $\mathrm{H}(28)$ & $C(16)$ & $H(29)$ & 109.48098 \\
\hline
\end{tabular}


Table 6. Bond angles involving hydrogens $\left({ }^{0}\right)$-- continued

$\begin{array}{llllllll}\text { atom } & \text { atom } & \text { atom } & \text { angle } & \text { atom } & \text { atom } & \text { atom } & \text { angle } \\ \mathrm{H}(28) & \mathrm{C}(16) & \mathrm{C}(14) & 110.037860 & \mathrm{H}(29) & \mathrm{C}(16) & \mathrm{C}(14) & 109.061617 \\ \mathrm{H}(4) & \mathrm{C}(3) & \mathrm{H}(5) & 109.460316 & \mathrm{H}(4) & \mathrm{C}(3) & \mathrm{H}(6) & 109.479690 \\ \mathrm{H}(4) & \mathrm{C}(3) & \mathrm{C}(1) & 109.332672 & \mathrm{H}(5) & \mathrm{C}(3) & \mathrm{H}(6) & 109.463013 \\ \mathrm{H}(5) & \mathrm{C}(3) & \mathrm{C}(1) & 109.613678 & \mathrm{H}(6) & \mathrm{C}(3) & \mathrm{C}(1) & 109.477842 \\ \mathrm{H}(1) & \mathrm{C}(2) & \mathrm{H}(2) & 109.467390 & \mathrm{H}(1) & \mathrm{C}(2) & \mathrm{H}(3) & 109.477355 \\ \mathrm{H}(1) & \mathrm{C}(2) & \mathrm{C}(1) & 108.525560 & \mathrm{H}(2) & \mathrm{C}(2) & \mathrm{H}(3) & 109.467126 \\ \mathrm{H}(2) & \mathrm{C}(2) & \mathrm{C}(1) & 109.278921 & \mathrm{H}(3) & \mathrm{C}(2) & \mathrm{C}(1) & 110.604154 \\ \mathrm{H}(24) & \mathrm{C}(15) & \mathrm{H}(25) & 109.477978 & \mathrm{H}(24) & \mathrm{C}(15) & \mathrm{H}(26) & 109.478897 \\ \mathrm{H}(24) & \mathrm{C}(15) & \mathrm{C}(14) & 109.234313 & \mathrm{H}(25) & \mathrm{C}(15) & \mathrm{H}(26) & 109.460148 \\ \mathrm{H}(25) & \mathrm{C}(15) & \mathrm{C}(14) & 109.786938 & \mathrm{H}(26) & \mathrm{C}(15) & \mathrm{C}(14) & 109.388587\end{array}$


Table 7. Torsion Angles( $\left({ }^{\circ}\right)$

atom1 atom2 atom3 atom4 angle

C(20) $\quad C(23) \quad C(24) \quad C(26)-175.6(6)$

$\mathrm{C}(20) \quad \mathrm{C}(23) \mathrm{C}(24) \quad \mathrm{H}(40) \quad 65.6(8)$

$\mathrm{C}(22) \quad \mathrm{C}(23) \quad \mathrm{C}(24) \quad \mathrm{C}(25)-175.2(5)$

$\begin{array}{lllll}\mathrm{H}(39) & \mathrm{C}(23) & \mathrm{C}(24) & \mathrm{C}(26) & -56.2(9)\end{array}$

$\begin{array}{lllll}\mathrm{H}(39) & \mathrm{C}(23) & \mathrm{C}(24) & \mathrm{H}(40) & -175.1(8)\end{array}$

$\begin{array}{lllll}\mathrm{C}(24) & \mathrm{C}(23) & \mathrm{C}(20) & \mathrm{H}(33) & 47.4(9)\end{array}$

$\mathrm{C}(22) \quad \mathrm{C}(23) \quad \mathrm{C}(20) \quad \mathrm{C}(19) \quad 51.4(8)$

$\begin{array}{lllll}\mathrm{C}(22) & \mathrm{C}(23) & \mathrm{C}(20) & \mathrm{H}(34) & -70.6(9)\end{array}$

$\begin{array}{lllll}\mathrm{H}(39) & \mathrm{C}(23) & \mathrm{C}(20) & \mathrm{H}(33) & -71(1)\end{array}$

C(24) C(23) C(22) C(21) 72.3(7)

$\begin{array}{lllll}\mathrm{C}(24) & \mathrm{C}(23) & \mathrm{C}(22) & \mathrm{H}(38) & -49.0(8)\end{array}$

$\mathrm{C}(20) \quad \mathrm{C}(23) \quad \mathrm{C}(22) \quad \mathrm{H}(37) \quad 68.9(8)$

$\begin{array}{lllll}\mathrm{H}(39) & \mathrm{C}(23) & \mathrm{C}(22) & \mathrm{C}(21) & -169.7(8)\end{array}$

$\mathrm{H}(39) \quad \mathrm{C}(23) \quad \mathrm{C}(22) \quad \mathrm{H}(38) \quad 68(1)$

$\begin{array}{lllll}\mathrm{C}(1) & \mathrm{C}(5) & \mathrm{C}(8) & \mathrm{H}(11) & -67.7(9)\end{array}$

$\begin{array}{lllll}\mathrm{C}(6) & \mathrm{C}(5) & \mathrm{C}(8) & \mathrm{C}(9) & -56.1(7)\end{array}$

$\begin{array}{lllll}\mathrm{C}(6) & \mathrm{C}(5) & \mathrm{C}(8) & \mathrm{H}(12) & -178.4(8)\end{array}$

$\mathrm{H}(10) \quad \mathrm{C}(5) \quad \mathrm{C}(8) \quad \mathrm{H}(11) \quad 175.3(9)$

$\begin{array}{lllll}\mathrm{C}(8) & \mathrm{C}(5) & \mathrm{C}(1) & \mathrm{C}(4) & 178.9(6)\end{array}$

$\begin{array}{lllll}\mathrm{C}(8) & \mathrm{C}(5) & \mathrm{C}(1) & \mathrm{C}(2) & -62.2(7)\end{array}$

$\begin{array}{lllll}\mathrm{C}(6) & \mathrm{C}(5) & \mathrm{C}(1) & \mathrm{C}(3) & -68.0(7)\end{array}$

$\begin{array}{lllll}\mathrm{H}(10) & \mathrm{C}(5) & \mathrm{C}(1) & \mathrm{C}(4) & -64.8(9)\end{array}$

$\begin{array}{lllll}\mathrm{H}(10) & \mathrm{C}(5) & \mathrm{C}(1) & \mathrm{C}(2) & 54.2(9)\end{array}$

$\begin{array}{lllll}\mathrm{C}(8) & \mathrm{C}(5) & \mathrm{C}(6) & \mathrm{H}(15) & 178.9(8)\end{array}$

$\begin{array}{lllll}C(1) & C(5) & C(6) & C(7) & -175.0(5)\end{array}$

$\begin{array}{lllll}\mathrm{C}(1) & \mathrm{C}(5) & \mathrm{C}(6) & \mathrm{H}(16) & 66.3(8)\end{array}$

$\begin{array}{lllll}H(10) & \mathrm{C}(5) & \mathrm{C}(6) & \mathrm{H}(15) & 66(1)\end{array}$

$\begin{array}{lllll}\mathrm{C}(5) & \mathrm{C}(8) & \mathrm{C}(9) & \mathrm{C}(10) & 57.0(7)\end{array}$

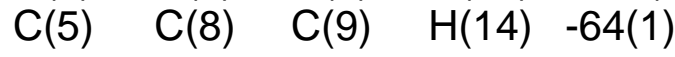

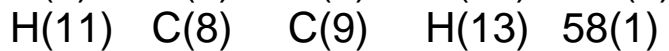

$\mathrm{H}(12) \quad \mathrm{C}(8) \quad \mathrm{C}(9) \quad \mathrm{C}(10) \quad 179.3(6)$

$\mathrm{H}(12) \quad \mathrm{C}(8) \quad \mathrm{C}(9) \quad \mathrm{H}(14) \quad 57(1)$

$\begin{array}{lllll}\mathrm{C}(18) & \mathrm{C}(19) & \mathrm{C}(20) & \mathrm{H}(33) & -174.9(8)\end{array}$

$\begin{array}{lllll}\mathrm{H}(31) & \mathrm{C}(19) & \mathrm{C}(20) & \mathrm{C}(23) & 62.1(9)\end{array}$

$\begin{array}{lllll}H(31) & \mathrm{C}(19) & \mathrm{C}(20) & \mathrm{H}(34) & -175.2(9)\end{array}$

$\mathrm{H}(32) \quad \mathrm{C}(19) \quad \mathrm{C}(20) \quad \mathrm{H}(33) \quad 63(1)$

C(20) C(19) C(18) C(21) $56.7(7)$ atom1 atom2 atom3 atom4 angle

$\begin{array}{lllll}C(20) & C(23) & C(24) & C(25) & -53.2(7)\end{array}$

C(22) $\quad \mathrm{C}(23) \quad \mathrm{C}(24) \quad \mathrm{C}(26) \quad 62.4(7)$

$\mathrm{C}(22) \quad \mathrm{C}(23) \mathrm{C}(24) \mathrm{H}(40)-56.5(7)$

$\begin{array}{lllll}\mathrm{H}(39) & \mathrm{C}(23) & \mathrm{C}(24) & \mathrm{C}(25) & 66.2(8)\end{array}$

$\begin{array}{lllll}\mathrm{C}(24) & \mathrm{C}(23) & \mathrm{C}(20) & \mathrm{C}(19) & -71.8(8)\end{array}$

$\begin{array}{lllll}\mathrm{C}(24) & \mathrm{C}(23) & \mathrm{C}(20) & \mathrm{H}(34) & 166.2(8)\end{array}$

$\begin{array}{lllll}\mathrm{C}(22) & \mathrm{C}(23) & \mathrm{C}(20) & \mathrm{H}(33) & 170.6(7)\end{array}$

$\begin{array}{lllll}\mathrm{H}(39) & \mathrm{C}(23) & \mathrm{C}(20) & \mathrm{C}(19) & 169.7(8)\end{array}$

$\mathrm{H}(39) \quad \mathrm{C}(23) \mathrm{C}(20) \mathrm{H}(34) \quad 47(1)$

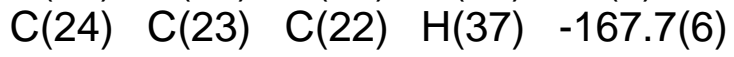

$\mathrm{C}(20) \quad \mathrm{C}(23) \quad \mathrm{C}(22) \quad \mathrm{C}(21) \quad-51.1(8)$

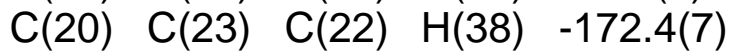

$\begin{array}{lllll}\mathrm{H}(39) & \mathrm{C}(23) & \mathrm{C}(22) & \mathrm{H}(37) & -49(1)\end{array}$

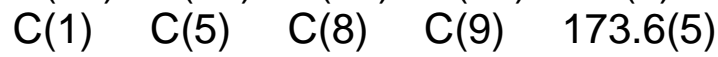

$\begin{array}{lllll}\mathrm{C}(1) & \mathrm{C}(5) & \mathrm{C}(8) & \mathrm{H}(12) & 51.2(9)\end{array}$

$\begin{array}{lllll}\mathrm{C}(6) & \mathrm{C}(5) & \mathrm{C}(8) & \mathrm{H}(11) & 62.7(9)\end{array}$

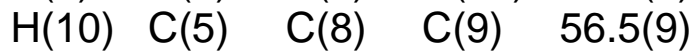

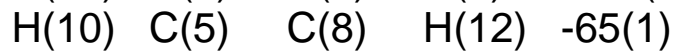

$\begin{array}{lllll}\mathrm{C}(8) & \mathrm{C}(5) & \mathrm{C}(1) & \mathrm{C}(3) & 57.5(8)\end{array}$

C(6) $\quad \mathrm{C}(5) \quad \mathrm{C}(1) \quad \mathrm{C}(4) \quad 53.3(7)$

$\begin{array}{lllll}\mathrm{C}(6) & \mathrm{C}(5) & \mathrm{C}(1) & \mathrm{C}(2) & 172.2(5)\end{array}$

$\begin{array}{lllll}\mathrm{H}(10) & \mathrm{C}(5) & \mathrm{C}(1) & \mathrm{C}(3) & 173.9(7)\end{array}$

$\begin{array}{lllll}\mathrm{C}(8) & \mathrm{C}(5) & \mathrm{C}(6) & \mathrm{C}(7) & 55.9(7)\end{array}$

$\begin{array}{lllll}\mathrm{C}(8) & \mathrm{C}(5) & \mathrm{C}(6) & \mathrm{H}(16) & -62.7(8)\end{array}$

$\begin{array}{lllll}\mathrm{C}(1) & \mathrm{C}(5) & \mathrm{C}(6) & \mathrm{H}(15) & -52(1)\end{array}$

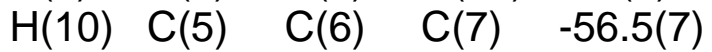

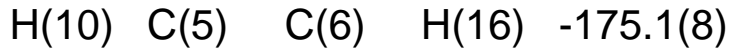

$\begin{array}{lllll}\mathrm{C}(5) & \mathrm{C}(8) & \mathrm{C}(9) & \mathrm{H}(13) & 177.1(7)\end{array}$

$\begin{array}{lllll}\mathrm{H}(11) & \mathrm{C}(8) & \mathrm{C}(9) & \mathrm{C}(10) & -61.1(9)\end{array}$

$\begin{array}{lllll}H(11) & \mathrm{C}(8) & \mathrm{C}(9) & \mathrm{H}(14) & 177.4(9)\end{array}$

$\mathrm{H}(12) \quad \mathrm{C}(8) \quad \mathrm{C}(9) \quad \mathrm{H}(13) \quad-60.6(9)$

$\begin{array}{lllll}C(18) & C(19) & C(20) & C(23) & -56.5(8)\end{array}$

$\begin{array}{lllll}\mathrm{C}(18) & \mathrm{C}(19) & \mathrm{C}(20) & \mathrm{H}(34) & 66.2(9)\end{array}$

$\begin{array}{lllll}H(31) & \mathrm{C}(19) & \mathrm{C}(20) & \mathrm{H}(33) & -56(1)\end{array}$

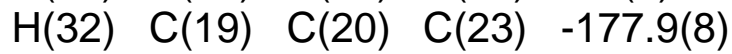

$\mathrm{H}(32) \quad \mathrm{C}(19) \quad \mathrm{C}(20) \quad \mathrm{H}(34)-55(1)$

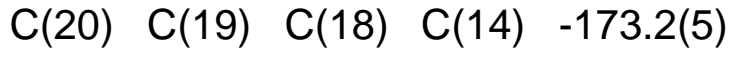


Table 7. Torsion angles (0) -- continued

atom1 atom2 atom3 atom4 angle

$\begin{array}{lllll}\mathrm{C}(20) & \mathrm{C}(19) & \mathrm{C}(18) & \mathrm{H}(30) & -56.1(7)\end{array}$

$\mathrm{H}(31) \quad \mathrm{C}(19) \quad \mathrm{C}(18) \quad \mathrm{C}(14) \quad 66.8(8)$

$\mathrm{H}(32) \quad \mathrm{C}(19) \quad \mathrm{C}(18) \quad \mathrm{C}(21) \quad 178.1(7)$

$\mathrm{H}(32) \quad \mathrm{C}(19) \quad \mathrm{C}(18) \quad \mathrm{H}(30) \quad 65.3(8)$

$\mathrm{C}(22) \quad \mathrm{C}(21) \quad \mathrm{C}(18) \quad \mathrm{C}(14) \quad 174.2(6)$

$\mathrm{H}(35) \quad \mathrm{C}(21) \quad \mathrm{C}(18) \quad \mathrm{C}(19) \quad 63(1)$

$\begin{array}{lllll}\mathrm{H}(35) & \mathrm{C}(21) & \mathrm{C}(18) & \mathrm{H}(30) & 176(1)\end{array}$

$\mathrm{H}(36) \quad \mathrm{C}(21) \quad \mathrm{C}(18) \quad \mathrm{C}(14) \quad 52.0(9)$

C(18) C(21) C(22) C(23) 55.2(9)

$\begin{array}{lllll}\mathrm{C}(18) & \mathrm{C}(21) & \mathrm{C}(22) & \mathrm{H}(38) & 176.3(8)\end{array}$

$\begin{array}{lllll}\mathrm{H}(35) & \mathrm{C}(21) & \mathrm{C}(22) & \mathrm{H}(37) & 175.8(9)\end{array}$

$\mathrm{H}(36) \quad \mathrm{C}(21) \quad \mathrm{C}(22) \quad \mathrm{C}(23) \quad 176.7(8)$

$\mathrm{H}(36) \quad \mathrm{C}(21) \quad \mathrm{C}(22) \quad \mathrm{H}(38)-62(1)$

$\begin{array}{lllll}C(17) & C(14) & C(18) & C(21) & -170.7(6)\end{array}$

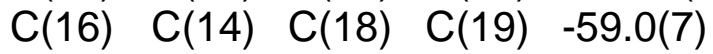

$\mathrm{C}(16) \quad \mathrm{C}(14) \mathrm{C}(18) \quad \mathrm{H}(30)-175.6(7)$

$\begin{array}{lllll}C(15) & C(14) & C(18) & C(21) & -52.4(8)\end{array}$

$\mathrm{C}(18) \quad \mathrm{C}(14) \quad \mathrm{C}(17) \quad \mathrm{H}(21) \quad-178(1)$

$\begin{array}{lllll}\mathrm{C}(18) & \mathrm{C}(14) & \mathrm{C}(17) & \mathrm{H}(23) & 60(1)\end{array}$

$\mathrm{C}(16) \quad \mathrm{C}(14) \quad \mathrm{C}(17) \mathrm{H}(22) \quad 63(1)$

C(15) $\mathrm{C}(14) \quad \mathrm{C}(17) \mathrm{H}(21) \quad 60(1)$

$\mathrm{C}(15) \quad \mathrm{C}(14) \quad \mathrm{C}(17) \quad \mathrm{H}(23) \quad-60(1)$

$\mathrm{C}(18) \quad \mathrm{C}(14) \mathrm{C}(16) \mathrm{H}(28) \quad-62(1)$

$\begin{array}{lllll}C(17) & C(14) & C(16) & H(27) & 56(1)\end{array}$

$\begin{array}{lllll}C(17) & C(14) & C(16) & H(29) & -63(1)\end{array}$

$\mathrm{C}(15) \quad \mathrm{C}(14) \quad \mathrm{C}(16) \mathrm{H}(28) \quad 59(1)$

$\mathrm{C}(18) \quad \mathrm{C}(14) \mathrm{C}(15) \mathrm{H}(24) \quad-58(1)$

C(18) $\mathrm{C}(14) \mathrm{C}(15) \quad \mathrm{H}(26)-178(1)$

$\mathrm{C}(17) \quad \mathrm{C}(14) \mathrm{C}(15) \quad \mathrm{H}(25)-179.3(8)$

$\begin{array}{lllll}\mathrm{C}(16) & \mathrm{C}(14) & \mathrm{C}(15) & \mathrm{H}(24) & 179.2(8)\end{array}$

$\mathrm{C}(16) \quad \mathrm{C}(14) \quad \mathrm{C}(15) \quad \mathrm{H}(26) \quad 59(1)$

$\begin{array}{lllll}\mathrm{C}(6) & \mathrm{C}(7) & \mathrm{C}(10) & \mathrm{C}(9) & 51.0(8)\end{array}$

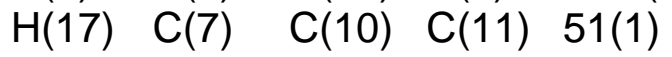

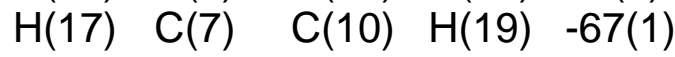

$\begin{array}{lllll}H(18) & \mathrm{C}(7) & \mathrm{C}(10) & \mathrm{C}(9) & -67(1)\end{array}$

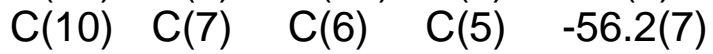

$\begin{array}{llll}\mathrm{C}(10) & \mathrm{C}(7) & \mathrm{C}(6) & \mathrm{H}(16)\end{array}$ atom1 atom2 atom3 atom4 angle

$\mathrm{H}(31) \quad \mathrm{C}(19) \quad \mathrm{C}(18) \quad \mathrm{C}(21) \quad-63.3(7)$

$\begin{array}{lllll}H(31) & \mathrm{C}(19) & \mathrm{C}(18) & \mathrm{H}(30) & -176.1(7)\end{array}$

$\mathrm{H}(32) \quad \mathrm{C}(19) \quad \mathrm{C}(18) \quad \mathrm{C}(14) \quad-51.8(9)$

$\begin{array}{lllll}\mathrm{C}(22) & \mathrm{C}(21) & \mathrm{C}(18) & \mathrm{C}(19) & -55.6(7)\end{array}$

$\mathrm{C}(22) \mathrm{C}(21) \mathrm{C}(18) \mathrm{H}(30) \quad 57(1)$

$\mathrm{H}(35) \quad \mathrm{C}(21) \quad \mathrm{C}(18) \quad \mathrm{C}(14)-66(1)$

$\begin{array}{lllll}H(36) & \mathrm{C}(21) & \mathrm{C}(18) & \mathrm{C}(19) & -177.8(7)\end{array}$

$\mathrm{H}(36) \quad \mathrm{C}(21) \quad \mathrm{C}(18) \quad \mathrm{H}(30)-65(1)$

$\begin{array}{lllll}\mathrm{C}(18) & \mathrm{C}(21) & \mathrm{C}(22) & \mathrm{H}(37) & -65.7(9)\end{array}$

$\mathrm{H}(35) \quad \mathrm{C}(21) \quad \mathrm{C}(22) \quad \mathrm{C}(23)-63(1)$

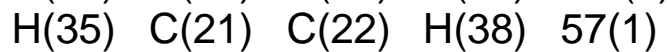

$\mathrm{H}(36) \quad \mathrm{C}(21) \quad \mathrm{C}(22) \quad \mathrm{H}(37) \quad 55(1)$

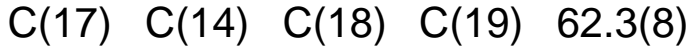

$\begin{array}{lllll}\mathrm{C}(17) & \mathrm{C}(14) & \mathrm{C}(18) & \mathrm{H}(30) & -54.3(8)\end{array}$

C(16) C(14) C(18) C(21) 68.1(7)

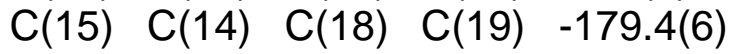

$\begin{array}{lllll}\mathrm{C}(15) & \mathrm{C}(14) & \mathrm{C}(18) & \mathrm{H}(30) & 64.0(8)\end{array}$

$\mathrm{C}(18) \quad \mathrm{C}(14) \mathrm{C}(17) \quad \mathrm{H}(22)-58(1)$

$\mathrm{C}(16) \quad \mathrm{C}(14) \quad \mathrm{C}(17) \quad \mathrm{H}(21)-57(1)$

C(16) $\mathrm{C}(14) \quad \mathrm{C}(17) \quad \mathrm{H}(23)-177(1)$

$\begin{array}{lllll}\mathrm{C}(15) & \mathrm{C}(14) & \mathrm{C}(17) & \mathrm{H}(22) & -179(359)\end{array}$

$\mathrm{C}(18) \quad \mathrm{C}(14) \quad \mathrm{C}(16) \mathrm{H}(27) \quad 177.0(9)$

$\mathrm{C}(18) \quad \mathrm{C}(14) \mathrm{C}(16) \mathrm{H}(29) \quad 57(1)$

$\mathrm{C}(17) \quad \mathrm{C}(14) \quad \mathrm{C}(16) \mathrm{H}(28) \quad 176.5(9)$

C(15) $\mathrm{C}(14) \quad \mathrm{C}(16) \quad \mathrm{H}(27)-60(1)$

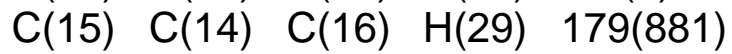

$\mathrm{C}(18) \quad \mathrm{C}(14) \quad \mathrm{C}(15) \quad \mathrm{H}(25) \quad 61(1)$

$\mathrm{C}(17) \quad \mathrm{C}(14) \mathrm{C}(15) \quad \mathrm{H}(24) \quad 60.6(9)$

$\begin{array}{lllll}\mathrm{C}(17) & \mathrm{C}(14) & \mathrm{C}(15) & \mathrm{H}(26) & -59(1)\end{array}$

$\begin{array}{lllll}\mathrm{C}(16) & \mathrm{C}(14) & \mathrm{C}(15) & \mathrm{H}(25) & -60.8(9)\end{array}$

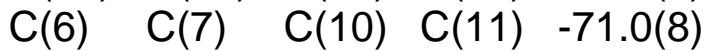

$\begin{array}{lllll}\mathrm{C}(6) & \mathrm{C}(7) & \mathrm{C}(10) & \mathrm{H}(19) & 170.5(8)\end{array}$

$\begin{array}{lllll}\mathrm{H}(17) & \mathrm{C}(7) & \mathrm{C}(10) & \mathrm{C}(9) & 173.3(9)\end{array}$

$\begin{array}{lllll}\mathrm{H}(18) & \mathrm{C}(7) & \mathrm{C}(10) & \mathrm{C}(11) & 170.8(9)\end{array}$

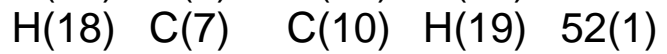

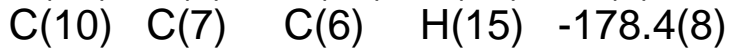

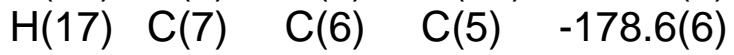


Table 7. Torsion angles (0) -- continued

atom1 atom2 atom3 atom4 angle

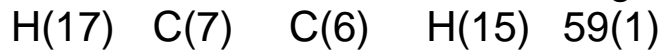

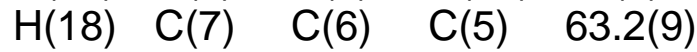

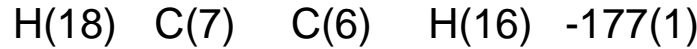

$\begin{array}{lllll}\mathrm{C}(5) & \mathrm{C}(1) & \mathrm{C}(4) & \mathrm{H}(8) & -60(1)\end{array}$

$\begin{array}{lllll}\mathrm{C}(3) & \mathrm{C}(1) & \mathrm{C}(4) & \mathrm{H}(7) & -57(1)\end{array}$

$\mathrm{C}(3) \quad \mathrm{C}(1) \quad \mathrm{C}(4) \quad \mathrm{H}(9) \quad-177.2(9)$

$\begin{array}{lllll}\mathrm{C}(2) & \mathrm{C}(1) & \mathrm{C}(4) & \mathrm{H}(8) & 178.4(9)\end{array}$

$\begin{array}{lllll}\mathrm{C}(5) & \mathrm{C}(1) & \mathrm{C}(3) & \mathrm{H}(4) & -61(1)\end{array}$

$\begin{array}{lllll}\mathrm{C}(5) & \mathrm{C}(1) & \mathrm{C}(3) & \mathrm{H}(6) & 178.5(9)\end{array}$

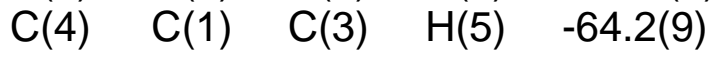

$\mathrm{C}(2) \quad \mathrm{C}(1) \quad \mathrm{C}(3) \quad \mathrm{H}(4) \quad 60(1)$

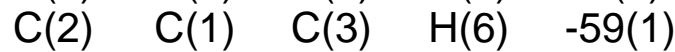

C(5) $\quad \mathrm{C}(1) \quad \mathrm{C}(2) \quad \mathrm{H}(2) \quad 60(1)$

$\mathrm{C}(4) \quad \mathrm{C}(1) \quad \mathrm{C}(2) \quad \mathrm{H}(1) \quad 61(1)$

$\mathrm{C}(4) \quad \mathrm{C}(1) \quad \mathrm{C}(2) \quad \mathrm{H}(3) \quad-58(1)$

$\mathrm{C}(3) \quad \mathrm{C}(1) \quad \mathrm{C}(2) \quad \mathrm{H}(2) \quad-62(1)$

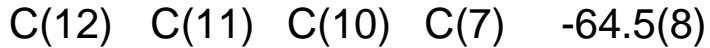

$\begin{array}{lllll}\mathrm{C}(12) & \mathrm{C}(11) & \mathrm{C}(10) & \mathrm{H}(19) & 54.7(9)\end{array}$

C(13) C(11) C(10) C(9) 52.5(8)

$\mathrm{H}(20) \quad \mathrm{C}(11) \quad \mathrm{C}(10) \mathrm{C}(7) \quad 53(1)$

$\mathrm{H}(20) \quad \mathrm{C}(11) \quad \mathrm{C}(10) \quad \mathrm{H}(19) \quad 173.2(9)$

$\begin{array}{lllll}\mathrm{C}(7) & \mathrm{C}(10) & \mathrm{C}(9) & \mathrm{H}(13) & -172.9(8)\end{array}$

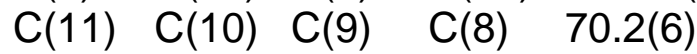

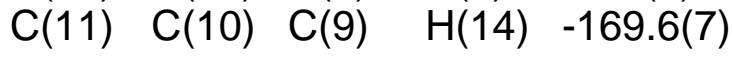

$\mathrm{H}(19) \quad \mathrm{C}(10) \quad \mathrm{C}(9) \quad \mathrm{H}(13) \quad 67(1)$ atom1 atom2 atom3 atom4 angle

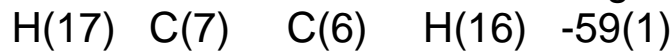

$\begin{array}{lllll}\mathrm{H}(18) & \mathrm{C}(7) & \mathrm{C}(6) & \mathrm{H}(15) & -58(1)\end{array}$

$\begin{array}{lllll}\mathrm{C}(5) & \mathrm{C}(1) & \mathrm{C}(4) & \mathrm{H}(7) & 179.0(9)\end{array}$

$\mathrm{C}(5) \quad \mathrm{C}(1) \quad \mathrm{C}(4) \quad \mathrm{H}(9) \quad 58(1)$

$\begin{array}{lllll}\mathrm{C}(3) & \mathrm{C}(1) & \mathrm{C}(4) & \mathrm{H}(8) & 63(1)\end{array}$

$\begin{array}{lllll}\mathrm{C}(2) & \mathrm{C}(1) & \mathrm{C}(4) & \mathrm{H}(7) & 57(1)\end{array}$

$\mathrm{C}(2) \quad \mathrm{C}(1) \quad \mathrm{C}(4) \quad \mathrm{H}(9) \quad-62(1)$

$\mathrm{C}(5) \quad \mathrm{C}(1) \quad \mathrm{C}(3) \quad \mathrm{H}(5) \quad 58.4(9)$

$\mathrm{C}(4) \quad \mathrm{C}(1) \quad \mathrm{C}(3) \quad \mathrm{H}(4) \quad 175.8(9)$

$\mathrm{C}(4) \quad \mathrm{C}(1) \quad \mathrm{C}(3) \quad \mathrm{H}(6) \quad 55(1)$

$\mathrm{C}(2) \quad \mathrm{C}(1) \quad \mathrm{C}(3) \quad \mathrm{H}(5) \quad-179.4(7)$

$\begin{array}{lllll}\mathrm{C}(5) & \mathrm{C}(1) & \mathrm{C}(2) & \mathrm{H}(1) & -59.0(9)\end{array}$

$\begin{array}{lllll}\mathrm{C}(5) & \mathrm{C}(1) & \mathrm{C}(2) & \mathrm{H}(3) & -179.1(9)\end{array}$

$\mathrm{C}(4) \quad \mathrm{C}(1) \quad \mathrm{C}(2) \quad \mathrm{H}(2) \quad-178(1)$

$\begin{array}{lllll}\mathrm{C}(3) & \mathrm{C}(1) & \mathrm{C}(2) & \mathrm{H}(1) & 177.8(8)\end{array}$

$\begin{array}{lllll}\mathrm{C}(3) & \mathrm{C}(1) & \mathrm{C}(2) & \mathrm{H}(3) & 57(1)\end{array}$

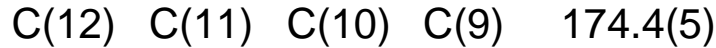

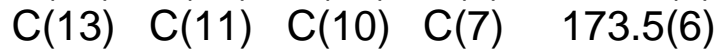

$\begin{array}{lllll}\mathrm{C}(13) & \mathrm{C}(11) & \mathrm{C}(10) & \mathrm{H}(19) & -67(1)\end{array}$

$\begin{array}{lllll}\mathrm{H}(20) & \mathrm{C}(11) & \mathrm{C}(10) & \mathrm{C}(9) & -67.2(8)\end{array}$

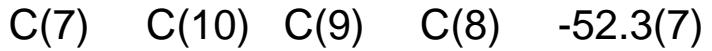

$\mathrm{C}(7) \quad \mathrm{C}(10) \quad \mathrm{C}(9) \quad \mathrm{H}(14) \quad 68.0(9)$

$\begin{array}{lllll}\mathrm{C}(11) & \mathrm{C}(10) & \mathrm{C}(9) & \mathrm{H}(13) & -50(1)\end{array}$

$\begin{array}{lllll}H(19) & \mathrm{C}(10) & \mathrm{C}(9) & \mathrm{C}(8) & -171.4(6)\end{array}$

$\mathrm{H}(19) \quad \mathrm{C}(10) \quad \mathrm{C}(9) \quad \mathrm{H}(14) \quad-51.1(9)$

The sign is positive if when looking from atom 2 to atom 3 a clock-wise motion of atom 1 would superimpose it on atom 4. 


\section{X-ray crystallographic Data of trans-6a}

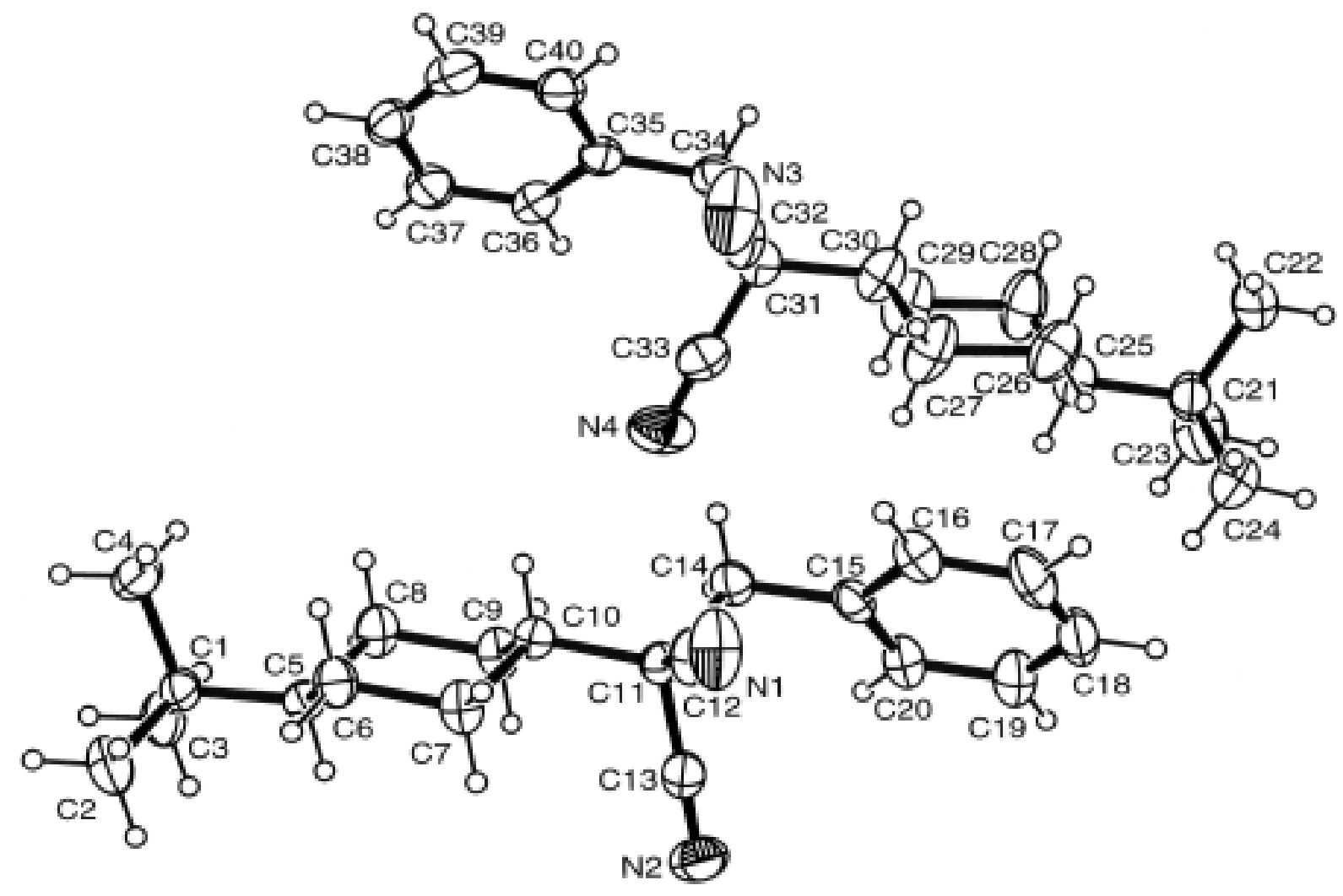

Figure S2. ORTEP drawing of trans-6a (ellipsoids at 30\% probability). 


\section{Experimental}

\section{$\underline{\text { Data Collection }}$}

A colorless prism crystal of $\mathrm{C}_{20} \mathrm{H}_{26} \mathrm{~N}_{2}$ having approximate dimensions of $0.80 \mathrm{x}$ $0.30 \times 0.20 \mathrm{~mm}$ was mounted on a glass fiber. All measurements were made on a Rigaku RAXIS RAPID imaging plate area detector with graphite monochromated Mo-Ka radiation.

Indexing was performed from 30 oscillations that were exposed for 180 seconds. The crystal-to-detector distance was $127.40 \mathrm{~mm}$.

Cell constants and an orientation matrix for data collection corresponded to a primitive monoclinic cell with dimensions:

$$
\begin{array}{lr}
\mathrm{a}= & 16.7104(3) \AA \\
\mathrm{b}= & 6.4424(1) \AA \quad \AA \quad \beta=110.457(2)^{\mathrm{O}} \\
\mathrm{c}= & 17.8094(4) \AA \\
\mathrm{V}=1796.36(6) \AA^{3}
\end{array}
$$

For $Z=4$ and F.W. $=294.44$, the calculated density is $1.09 \mathrm{~g} / \mathrm{cm}^{3}$. Based on the systematic absences of:

Ok0: $\quad k \pm 2 n$

packing considerations, a statistical analysis of intensity distribution, and the successful solution and refinement of the structure, the space group was determined to be:

$$
\text { P21 (\#4) }
$$

The data were collected at a temperature of $23 \pm 1^{\circ} \mathrm{C}$ to a maximum $2 \theta$ value of 55.00. A total of 44 oscillation images were collected. A sweep of data was done using $\omega$ scans from 130.0 to $190.0^{\circ}$ in $5.0^{\circ}$ step, at $\chi=45.0^{\circ}$ and $\phi=0.0^{\circ}$. The exposure rate was 300.0 [sec./0]. A second sweep was performed using $\omega$ scans from 0.0 to $160.0^{0}$ in $5.0^{\circ}$ step, at $\chi=45.0^{\circ}$ and $\phi=180.0^{\circ}$. The exposure rate was 300.0 [sec./0]. The 
crystal-to-detector distance was $127.40 \mathrm{~mm}$. Readout was performed in the $0.100 \mathrm{~mm}$ pixel mode.

\section{Data Reduction}

Of the 4342 reflections that were collected, 4338 were unique $\left(R_{\text {int }}=0.049\right)$; equivalent reflections were merged.

The linear absorption coefficient, $\mu$, for Mo-K $\alpha$ radiation is $0.6 \mathrm{~cm}^{-1}$. The data were corrected for Lorentz and polarization effects.

\section{$\underline{\text { Structure Solution and Refinement }}$}

The structure was solved by direct methods ${ }^{1}$ and expanded using Fourier techniques $^{2}$. The non-hydrogen atoms were refined anisotropically. Hydrogen atoms were refined using the riding model. The final cycle of full-matrix least-squares refinement 3 on $F$ was based on 3823 observed reflections $(I>0.50 \sigma(I))$ and 449 variable parameters and converged (largest parameter shift was 0.00 times its esd) with unweighted and weighted agreement factors of:

$$
\begin{gathered}
\mathrm{R}=\Sigma|| \mathrm{Fo}|-| \mathrm{Fc}|| / \Sigma|\mathrm{Fo}|=0.079 \\
\mathrm{R}_{\mathrm{W}}=\left[\Sigma \mathrm{w}(|\mathrm{Fo}|-|\mathrm{Fc}|)^{2} / \Sigma \mathrm{w} \mathrm{Fo}{ }^{2}\right]^{1 / 2}=0.136
\end{gathered}
$$

The standard deviation of an observation of unit weight ${ }^{4}$ was 1.03. A Sheldrick weighting scheme was used. Plots of $\Sigma \mathrm{w}(|\mathrm{Fo}|-|\mathrm{Fc}|)^{2}$ versus $|\mathrm{Fo}|$, reflection order in data collection, $\sin \theta / \lambda$ and various classes of indices showed no unusual trends. The maximum and minimum peaks on the final difference Fourier map corresponded to 0.95 and $-0.47 \mathrm{e}^{-} / \AA^{3}$, respectively.

Neutral atom scattering factors were taken from Cromer and Waber 5 . Anomalous dispersion effects were included in Fcalc 6 ; the values for $\Delta f^{\prime}$ and $\Delta f^{\prime \prime}$ were those of Creagh and McAuley7. The values for the mass attenuation coefficients are those of Creagh and Hubbell 8 . All calculations were performed using the CrystalStructure 9,10 crystallographic software package. 
References

(1) SIR92: Altomare, A., Cascarano, G., Giacovazzo, C., Guagliardi, A., Burla, M., Polidori, G., and Camalli, M. (1994) J. Appl. Cryst., 27, 435.

(2) DIRDIF99: Beurskens, P.T., Admiraal, G., Beurskens, G., Bosman, W.P., de Gelder, R., Israel, R. and Smits, J.M.M.(1999). The DIRDIF-99 program system, Technical Report of the Crystallography Laboratory, University of Nijmegen, The Netherlands.

(3) Least Squares function minimized:

$$
\Sigma w\left(\left|F_{\mathrm{o}}\right|-\left|\mathrm{F}_{\mathrm{c}}\right|\right)^{2} \quad \text { where } \mathrm{w}=\text { Least Squares weights. }
$$

(4) Standard deviation of an observation of unit weight:

$$
\begin{aligned}
& {\left[\Sigma w\left(\left|\mathrm{~F}_{\mathrm{O}}\right|-\left|\mathrm{F}_{\mathrm{C}}\right|\right)^{2} /\left(\mathrm{N}_{\mathrm{O}}-\mathrm{N}_{\mathrm{V}}\right)\right]^{1 / 2}} \\
& \text { where: } \quad \mathrm{N}_{\mathrm{O}}=\text { number of observations } \\
& \mathrm{N}_{\mathrm{V}}=\text { number of variables }
\end{aligned}
$$

(5) Cromer, D. T. \& Waber, J. T.; "International Tables for X-ray Crystallography", Vol. IV, The Kynoch Press, Birmingham, England, Table 2.2 A (1974).

(6) Ibers, J. A. \& Hamilton, W. C.; Acta Crystallogr., 17, 781 (1964).

(7) Creagh, D. C. \& McAuley, W.J .; "International Tables for Crystallography", Vol C, (A.J.C. Wilson, ed.), Kluwer Academic Publishers, Boston, Table 4.2.6.8, pages 219-222 (1992).

(8) Creagh, D. C. \& Hubbell, J.H..; "International Tables for Crystallography", Vol C, (A.J.C. Wilson, ed.), Kluwer Academic Publishers, Boston, Table 4.2.4.3, pages 200-206 (1992).

(9) CrystalStructure 3.5.1: Crystal Structure Analysis Package, Rigaku and Rigaku/MSC (2000-2003). 9009 New Trails Dr. The Woodlands TX 77381 USA.

(10) CRYSTALS Issue 10: Watkin, D.J., Prout, C.K. Carruthers, J.R. \& Betteridge, P.W. Chemical Crystallography Laboratory, Oxford, UK. (1996) 
EXPERIMENTAL DETAILS

\author{
A. Crystal Data
}

Empirical Formula

Formula Weight

Crystal Color, Habit

Crystal Dimensions

Crystal System

Lattice Type

Indexing Images

Detector Position

Pixel Size

Lattice Parameters

Space Group

$Z$ value

$D_{\text {calc }}$

$F_{000}$
$\mathrm{C}_{20} \mathrm{H}_{26} \mathrm{~N}_{2}$

294.44

colorless, prism

$0.80 \times 0.30 \times 0.20 \mathrm{~mm}$

monoclinic

Primitive

3 oscillations @ 180.0 seconds

$127.40 \mathrm{~mm}$

$0.100 \mathrm{~mm}$

$$
\begin{aligned}
& \mathrm{a}=16.7104(3) \AA \\
& \mathrm{b}=6.4424(1) \AA \\
& \mathrm{c}=17.8094(4) \AA \\
& \beta=110.457(2) \AA \\
& \mathrm{V}=1796.36(6) \AA^{3} \\
& \mathrm{P} 21(\# 4) \\
& 4 \\
& 1.089 \mathrm{~g} / \mathrm{cm}^{3} \\
& 640.00
\end{aligned}
$$


$\mu(\operatorname{MoK} \alpha)$

$0.64 \mathrm{~cm}^{-1}$ 
B. Intensity Measurements

Diffractometer

Radiation

Detector Aperture

Data Images

$\omega$ oscillation Range $(\chi=45.0, \phi=0.0)$

Exposure Rate

$\omega$ oscillation Range ( $\chi=45.0, \phi=180.0)$

Exposure Rate

Detector Position

Pixel Size

$2 \theta_{\max }$

No. of Reflections Measured

Corrections
Rigaku RAXIS-RAPID

$\operatorname{MoK} \alpha(\lambda=0.71069 \AA)$

graphite monochromated

$270 \mathrm{~mm} \times 256 \mathrm{~mm}$

44 exposures

$130.0-190.00$

$300.0 \mathrm{sec} . / 0$

$0.0-160.00$

$300.0 \mathrm{sec} . / 0$

$127.40 \mathrm{~mm}$

$0.100 \mathrm{~mm}$

$55.0^{\circ}$

Total: 4342

Unique: $4338\left(R_{\text {int }}=0.049\right)$ Lorentz-polarization 
C. Structure Solution and Refinement

Structure Solution

Refinement

Function Minimized

Least Squares Weights

Anomalous Dispersion

No. Observations $(\mathrm{I}>0.50 \sigma(\mathrm{I}))$

No. Variables

Reflection/Parameter Ratio

Residuals: $\mathrm{R}(\mathrm{I}>0.50 \sigma(\mathrm{I}))$

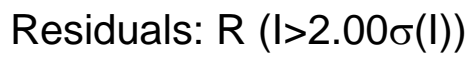

Residuals: Rw $(I>0.50 \sigma(I))$

Goodness of Fit Indicator

Max Shift/Error in Final Cycle

Maximum peak in Final Diff. Map

Minimum peak in Final Diff. Map
Direct Methods (SIR92)

Full-matrix least-squares on $\mathrm{F}$

$\Sigma \mathrm{w}(|\mathrm{Fo}|-|\mathrm{Fc}|)^{2}$

$1 /\left[0.0010 \mathrm{Fo}^{2}+1.0000 \sigma\left(\mathrm{Fo}^{2}\right)+0.1000\right]$

All non-hydrogen atoms

3823

449

8.51

0.094

0.079

0.136

1.030

0.000

$0.95 \mathrm{e}^{-/ \AA^{3}}$

$-0.47 e^{-} / \AA^{3}$ 
Table 1. Atomic coordinates and $\mathrm{B}_{\text {iso }} / \mathrm{B}_{\text {eq }}$

$\begin{array}{lcccc}\text { atom } & x & y & z & B_{\text {eq }} \\ \mathrm{N}(1) & 1.4016 & -0.7492 & 0.2798 & 6.819 \\ \mathrm{~N}(2) & 1.5370 & -0.1892 & 0.3052 & 6.056 \\ \mathrm{~N}(3) & 1.0348 & -0.4397 & 0.2213 & 8.985 \\ \mathrm{~N}(4) & 1.1613 & 0.1164 & 0.2049 & 7.658 \\ \mathrm{C}(5) & 1.3708 & -0.2095 & -0.0016 & 3.509 \\ \mathrm{C}(7) & 1.4056 & -0.4574 & 0.1147 & 4.069 \\ \mathrm{C}(11) & 1.3850 & -0.3541 & 0.2449 & 3.349 \\ \mathrm{C}(14) & 1.3228 & -0.2602 & 0.2834 & 3.966 \\ \mathrm{C}(10) & 1.3511 & -0.3315 & 0.1521 & 3.368 \\ \mathrm{C}(13) & 1.4714 & -0.2603 & 0.2799 & 4.049 \\ \mathrm{C}(9) & 1.3466 & -0.1066 & 0.1258 & 4.330 \\ \mathrm{C}(8) & 1.3150 & -0.0890 & 0.0346 & 4.661 \\ \mathrm{C}(33) & 1.1089 & 0.0485 & 0.2237 & 5.224 \\ \mathrm{C}(34) & 0.9529 & 0.0520 & 0.2001 & 4.455 \\ \mathrm{C}(3) & 1.3566 & 0.0543 & -0.1091 & 5.236 \\ \mathrm{C}(15) & 1.3507 & -0.2812 & 0.3732 & 3.914 \\ \mathrm{C}(1) & 1.3479 & -0.1759 & -0.0923 & 4.108 \\ \mathrm{C}(40) & 0.8848 & -0.1479 & 0.0724 & 4.949 \\ \mathrm{C}(31) & 1.0415 & -0.0414 & 0.2496 & 4.339 \\ \mathrm{C}(38) & 0.8804 & -0.0152 & -0.0524 & 5.621 \\ \mathrm{C}(20) & 1.3965 & -0.1278 & 0.4222 & 4.952 \\ \mathrm{C}(35) & 0.9267 & 0.0252 & 0.1115 & 4.215 \\ \mathrm{C}(12) & 1.3954 & -0.5804 & 0.2653 & 4.569 \\ \mathrm{C}(19) & 1.4228 & -0.1495 & 0.5048 & 5.765 \\ \mathrm{C}(16) & 1.3323 & -0.4586 & 0.4079 & 5.083 \\ \mathrm{C}(37) & 0.9210 & 0.1616 & -0.0158 & 5.342 \\ \mathrm{C}(36) & 0.9441 & 0.1811 & 0.0669 & 4.613 \\ \mathrm{C}(39) & 0.8619 & -0.1682 & -0.0087 & 5.877 \\ \mathrm{C}(4) & 1.2569 & -0.2481 & -0.1406 & 6.113 \\ \mathrm{C}(6) & 1.3724 & -0.4338 & 0.0237 & 4.246 \\ \mathrm{C}(29) & 1.0703 & 0.1959 & 0.3704 & 6.945 \\ \mathrm{C}(2) & 1.4080 & -0.2986 & -0.1225 & 5.462 \\ \mathrm{C}(18) & 1.4047 & -0.3206 & 0.5388 & 6.111 \\ \mathrm{C}(21) & 1.1892 & 0.1091 & 0.6010 & 5.060 \\ \mathrm{C}(25) & 1.1648 & 0.0842 & 0.5104 & 5.213 \\ \mathrm{C}(26) & 1.1558 & -0.1356 & 0.4830 & 6.945 \\ \mathrm{C}(24) & 1.2748 & -0.0026 & 0.6440 & 6.742\end{array}$


Table 1. Atomic coordinates and $\mathrm{B}_{\mathrm{iso}} / \mathrm{B}_{\mathrm{eq}}$ (continued)

$\begin{array}{lcccc}\text { atom } & \mathrm{x} & \mathrm{y} & \mathrm{z} & \mathrm{B} \\ \mathrm{C}(22) & 1.1228 & 0.0182 & 0.6320 & 6.667 \\ \mathrm{C}(27) & 1.1422 & -0.1441 & 0.3906 & 7.501 \\ \mathrm{C}(30) & 1.0627 & -0.0211 & 0.3428 & 5.494 \\ \mathrm{C}(17) & 1.3616 & -0.4744 & 0.4921 & 6.274 \\ \mathrm{C}(32) & 1.0409 & -0.2670 & 0.2330 & 5.620 \\ \mathrm{C}(28) & 1.0844 & 0.2018 & 0.4611 & 7.814 \\ \mathrm{C}(23) & 1.2030 & 0.3379 & 0.6246 & 8.400 \\ \mathrm{H}(1) & 1.4031 & -0.4423 & -0.1130 & 6.947 \\ \mathrm{H}(2) & 1.3949 & -0.2759 & -0.1782 & 6.946 \\ \mathrm{H}(3) & 1.4647 & -0.2538 & -0.0940 & 6.947 \\ \mathrm{H}(4) & 1.4131 & 0.0978 & -0.0793 & 6.377 \\ \mathrm{H}(5) & 1.3455 & 0.0750 & -0.1646 & 6.377 \\ \mathrm{H}(6) & 1.3172 & 0.1332 & -0.0931 & 6.379 \\ \mathrm{H}(7) & 1.2177 & -0.1694 & -0.1244 & 7.005 \\ \mathrm{H}(8) & 1.2459 & -0.2261 & -0.1960 & 7.003 \\ \mathrm{H}(9) & 1.2505 & -0.3913 & -0.1313 & 7.007 \\ \mathrm{H}(10) & 1.4267 & -0.1556 & 0.0235 & 4.177 \\ \mathrm{H}(11) & 1.3168 & -0.4912 & 0.0013 & 5.197 \\ \mathrm{H}(12) & 1.4104 & -0.5073 & 0.0042 & 5.198 \\ \mathrm{H}(13) & 1.4630 & -0.4102 & 0.1364 & 4.997 \\ \mathrm{H}(14) & 1.4031 & -0.5997 & 0.1277 & 5.000 \\ \mathrm{H}(15) & 1.3125 & 0.0528 & 0.0193 & 5.571 \\ \mathrm{H}(16) & 1.2594 & -0.1475 & 0.0141 & 5.573 \\ \mathrm{H}(17) & 1.3094 & -0.0316 & 0.1459 & 5.318 \\ \mathrm{H}(18) & 1.4023 & -0.0485 & 0.1466 & 5.320 \\ \mathrm{H}(19) & 1.2946 & -0.3852 & 0.1323 & 4.031 \\ \mathrm{H}(20) & 1.3162 & -0.1166 & 0.2706 & 4.857 \\ \mathrm{H}(21) & 1.2692 & -0.3280 & 0.2609 & 4.858 \\ \mathrm{H}(22) & 1.3012 & -0.5687 & 0.3751 & 6.433 \\ \mathrm{H}(23) & 1.3490 & -0.5958 & 0.5161 & 8.118 \\ \mathrm{H}(24) & 1.4226 & -0.3332 & 0.5954 & 7.618 \\ \mathrm{H}(25) & 1.4553 & -0.0429 & 0.5387 & 6.981 \\ \mathrm{H}(26) & 1.4104 & -0.0063 & 0.3991 & 6.165 \\ \mathrm{H}(27) & 1.1384 & 0.0413 & 0.6880 & 8.174 \\ \mathrm{H}(28) & 1.0698 & 0.0841 & 0.6046 & 8.174 \\ \mathrm{H}(29) & 1.1177 & -0.1267 & 0.6215 & 8.174 \\ \mathrm{H}(30) & 1.2246 & 0.3520 & 0.6812 & 10.257 \\ & & & & \end{array}$


Table 1. Atomic coordinates and $\mathrm{B}_{\mathrm{iso}} / \mathrm{B}_{\text {eq }}$ (continued)

$\begin{array}{lcccl}\text { atom } & x & y & z & B_{\text {eq }} \\ \mathrm{H}(31) & 1.2453 & 0.3810 & 0.6035 & 10.257 \\ \mathrm{H}(32) & 1.1535 & 0.4219 & 0.6028 & 10.253 \\ \mathrm{H}(33) & 1.2654 & -0.1466 & 0.6328 & 7.720 \\ \mathrm{H}(34) & 1.3162 & 0.0465 & 0.6228 & 7.718 \\ \mathrm{H}(35) & 1.2949 & 0.0187 & 0.7003 & 7.718 \\ \mathrm{H}(36) & 1.2113 & 0.1407 & 0.4982 & 6.063 \\ \mathrm{H}(37) & 1.2072 & -0.2064 & 0.5117 & 7.625 \\ \mathrm{H}(38) & 1.1094 & -0.2005 & 0.4929 & 7.623 \\ \mathrm{H}(39) & 1.1905 & -0.0891 & 0.3808 & 8.309 \\ \mathrm{H}(40) & 1.1347 & -0.2854 & 0.3745 & 8.313 \\ \mathrm{H}(41) & 1.0941 & 0.3419 & 0.4786 & 9.171 \\ \mathrm{H}(42) & 1.0350 & 0.1509 & 0.4697 & 9.168 \\ \mathrm{H}(43) & 1.1176 & 0.2575 & 0.3610 & 7.814 \\ \mathrm{H}(44) & 1.0197 & 0.2700 & 0.3417 & 7.816 \\ \mathrm{H}(45) & 1.0157 & -0.0791 & 0.3539 & 6.188 \\ \mathrm{H}(46) & 0.9110 & -0.0173 & 0.2157 & 5.334 \\ \mathrm{H}(47) & 0.9531 & 0.1955 & 0.2124 & 5.331 \\ \mathrm{H}(48) & 0.9720 & 0.3041 & 0.0924 & 5.357 \\ \mathrm{H}(49) & 0.9334 & 0.2694 & -0.0464 & 6.379 \\ \mathrm{H}(50) & 0.8660 & -0.0292 & -0.1087 & 6.548 \\ \mathrm{H}(51) & 0.8328 & -0.2888 & -0.0353 & 6.775 \\ \mathrm{H}(52) & 0.8714 & -0.2550 & 0.1027 & 5.938\end{array}$

$B_{e q}=8 / 3 \pi^{2}\left(U_{11}\left(a a^{\star}\right)^{2}+U_{22}\left(b b^{\star}\right)^{2}+U_{33}\left(c c^{\star}\right)^{2}+2 U_{12}\left(a a^{*} b b^{\star}\right) \cos \gamma+2 U_{13}\left(a a^{*} c^{*}\right) \cos \beta+2 U_{23}\left(b b^{*} c c^{\star}\right) \cos \alpha\right)$ 
Table 2. Anisotropic Displacement Parameters

\begin{tabular}{|c|c|c|c|c|c|c|}
\hline atom & $U_{11}$ & $\mathrm{U}_{22}$ & $\mathrm{U}_{33}$ & $U_{12}$ & $U_{13}$ & $\mathrm{U}_{23}$ \\
\hline$N(1)$ & 0.1557 & 0.0422 & 0.0650 & 0.0154 & 0.0433 & 0.0076 \\
\hline $\mathrm{N}(2)$ & 0.0515 & 0.0907 & 0.0816 & -0.0034 & 0.0154 & -0.0219 \\
\hline$N(3)$ & 0.2048 & 0.0530 & 0.0762 & 0.0144 & 0.0398 & 0.0027 \\
\hline $\mathrm{N}(4)$ & 0.0612 & 0.1002 & 0.1267 & 0.0226 & 0.0292 & 0.0396 \\
\hline C(5) & 0.0434 & 0.0452 & 0.0439 & -0.0027 & 0.0141 & -0.0018 \\
\hline C(7) & 0.0724 & 0.0386 & 0.0473 & 0.0128 & 0.0255 & 0.0030 \\
\hline$C(11)$ & 0.0455 & 0.0403 & 0.0411 & 0.0044 & 0.0147 & 0.0036 \\
\hline$C(14)$ & 0.0511 & 0.0519 & 0.0508 & 0.0113 & 0.0217 & 0.0046 \\
\hline$C(10)$ & 0.0461 & 0.0393 & 0.0423 & 0.0031 & 0.0151 & -0.0007 \\
\hline$C(13)$ & 0.0555 & 0.0512 & 0.0474 & 0.0159 & 0.0184 & 0.0019 \\
\hline C(9) & 0.0719 & 0.0454 & 0.0511 & 0.0133 & 0.0264 & 0.0051 \\
\hline$C(8)$ & 0.0716 & 0.0559 & 0.0490 & 0.0210 & 0.0202 & 0.0078 \\
\hline C(33) & 0.0556 & 0.0611 & 0.0732 & 0.0188 & 0.0117 & 0.0101 \\
\hline$C(34)$ & 0.0480 & 0.0581 & 0.0628 & 0.0042 & 0.0187 & -0.0057 \\
\hline$C(3)$ & 0.0925 & 0.0549 & 0.0545 & -0.0027 & 0.0295 & 0.0096 \\
\hline$C(15)$ & 0.0497 & 0.0550 & 0.0518 & 0.0137 & 0.0275 & 0.0127 \\
\hline$C(1)^{\prime}$ & 0.0549 & 0.0590 & 0.0426 & -0.0118 & 0.0175 & 0.0001 \\
\hline C(40) & 0.0545 & 0.0655 & 0.0679 & -0.0080 & 0.0211 & -0.0040 \\
\hline$C(31)$ & 0.0628 & 0.0484 & 0.0530 & 0.0097 & 0.0193 & -0.0002 \\
\hline$C(38)$ & 0.0502 & 0.1022 & 0.0549 & 0.0100 & 0.0106 & -0.0123 \\
\hline$C(20)$ & 0.0807 & 0.0621 & 0.0524 & 0.0073 & 0.0320 & 0.0068 \\
\hline$C(35)$ & 0.0430 & 0.0629 & 0.0539 & 0.0050 & 0.0164 & -0.0018 \\
\hline$C(12)$ & 0.0777 & 0.0528 & 0.0437 & 0.0118 & 0.0221 & 0.0006 \\
\hline$C(19)$ & 0.0887 & 0.0801 & 0.0521 & 0.0053 & 0.0270 & -0.0006 \\
\hline$C(16)$ & 0.0758 & 0.0612 & 0.0667 & -0.0004 & 0.0382 & 0.0062 \\
\hline$C(37)$ & 0.0572 & 0.0801 & 0.0646 & 0.0044 & 0.0199 & 0.0109 \\
\hline$C(36)$ & 0.0523 & 0.0578 & 0.0596 & 0.0010 & 0.0126 & -0.0046 \\
\hline C(39) & 0.0519 & 0.0794 & 0.0832 & -0.0025 & 0.0126 & -0.0236 \\
\hline C(4) & 0.0630 & 0.1076 & 0.0512 & -0.0280 & 0.0070 & 0.0074 \\
\hline$C(6)$ & 0.0666 & 0.0479 & 0.0500 & 0.0026 & 0.0244 & -0.0042 \\
\hline$C(29)$ & 0.1245 & 0.0510 & 0.0718 & 0.0239 & 0.0135 & 0.0031 \\
\hline$C(2)$ & 0.0933 & 0.0683 & 0.0584 & -0.0062 & 0.0421 & -0.0016 \\
\hline$C(18)$ & 0.0881 & 0.1010 & 0.0521 & 0.0211 & 0.0359 & 0.0182 \\
\hline$C(21)$ & 0.0750 & 0.0692 & 0.0471 & -0.0101 & 0.0200 & -0.0001 \\
\hline$C(25)$ & 0.0729 & 0.0659 & 0.0531 & 0.0066 & 0.0144 & 0.0052 \\
\hline$C(26)$ & 0.1026 & 0.0713 & 0.0675 & 0.0075 & 0.0014 & 0.0124 \\
\hline$C(24)$ & 0.0788 & 0.1070 & 0.0587 & -0.0032 & 0.0093 & -0.0029 \\
\hline
\end{tabular}


Table 2. Anisotropic Displacement Parameters (continued)

$\begin{array}{lcccccc}\text { atom } & \mathrm{U}_{11} & \mathrm{U}_{22} & \mathrm{U}_{33} & \mathrm{U}_{12} & \mathrm{U}_{13} & \mathrm{U}_{23} \\ \mathrm{C}(22) & 0.0936 & 0.1045 & 0.0608 & -0.0077 & 0.0340 & -0.0038 \\ \mathrm{C}(27) & 0.1052 & 0.0950 & 0.0631 & 0.0530 & 0.0020 & -0.0099 \\ \mathrm{C}(30) & 0.0744 & 0.0631 & 0.0584 & 0.0204 & 0.0071 & -0.0082 \\ \mathrm{C}(17) & 0.1010 & 0.0796 & 0.0765 & 0.0162 & 0.0544 & 0.0319 \\ \mathrm{C}(32) & 0.1032 & 0.0541 & 0.0547 & 0.0130 & 0.0257 & 0.0046 \\ \mathrm{C}(28) & 0.1310 & 0.0993 & 0.0600 & 0.0469 & 0.0251 & -0.0070 \\ \mathrm{C}(23) & 0.1545 & 0.0811 & 0.0892 & -0.0183 & 0.0496 & -0.0173\end{array}$

The general temperature factor expression: $\exp \left(-2 \pi^{2}\left(a^{\star 2} U_{11} h^{2}+b^{*} 2 U_{22} k^{2}+\left.c^{\star 2} U_{33}\right|^{2}\right.\right.$ $\left.\left.+2 a^{*} b^{*} U_{12} h k+2 a^{*} c^{*} U_{13} h l+2 b^{*} c^{*} U_{23} k l\right)\right)$ 
Table 3. Bond lengths $(\AA ̊)$

$\begin{array}{llllll}\text { atom } & \text { atom } & \text { distance } & \text { atom } & \text { atom } & \text { distance } \\ \mathrm{N}(1) & \mathrm{C}(12) & 1.11(1) & \mathrm{N}(2) & \mathrm{C}(13) & 1.13(1) \\ \mathrm{N}(3) & \mathrm{C}(32) & 1.13(1) & \mathrm{N}(4) & \mathrm{C}(33) & 1.13(1) \\ \mathrm{C}(5) & \mathrm{C}(8) & 1.52(1) & \mathrm{C}(5) & \mathrm{C}(1) & 1.54(1) \\ \mathrm{C}(5) & \mathrm{C}(6) & 1.51(1) & \mathrm{C}(7) & \mathrm{C}(10) & 1.54(1) \\ \mathrm{C}(7) & \mathrm{C}(6) & 1.53(1) & \mathrm{C}(11) & \mathrm{C}(14) & 1.55(1) \\ \mathrm{C}(11) & \mathrm{C}(10) & 1.555(9) & \mathrm{C}(11) & \mathrm{C}(13) & 1.49(1) \\ \mathrm{C}(11) & \mathrm{C}(12) & 1.50(1) & \mathrm{C}(14) & \mathrm{C}(15) & 1.51(1) \\ \mathrm{C}(10) & \mathrm{C}(9) & 1.52(1) & \mathrm{C}(9) & \mathrm{C}(8) & 1.53(1) \\ \mathrm{C}(33) & \mathrm{C}(31) & 1.48(1) & \mathrm{C}(34) & \mathrm{C}(31) & 1.56(1) \\ \mathrm{C}(34) & \mathrm{C}(35) & 1.49(1) & \mathrm{C}(3) & \mathrm{C}(1) & 1.53(1) \\ \mathrm{C}(15) & \mathrm{C}(20) & 1.36(1) & \mathrm{C}(15) & \mathrm{C}(16) & 1.38(1) \\ \mathrm{C}(1) & \mathrm{C}(4) & 1.53(1) & \mathrm{C}(1) & \mathrm{C}(2) & 1.52(1) \\ \mathrm{C}(40) & \mathrm{C}(35) & 1.37(1) & \mathrm{C}(40) & \mathrm{C}(39) & 1.36(1) \\ \mathrm{C}(31) & \mathrm{C}(30) & 1.58(1) & \mathrm{C}(31) & \mathrm{C}(32) & 1.48(1) \\ \mathrm{C}(38) & \mathrm{C}(37) & 1.37(1) & \mathrm{C}(38) & \mathrm{C}(39) & 1.36(2) \\ \mathrm{C}(20) & \mathrm{C}(19) & 1.39(1) & \mathrm{C}(35) & \mathrm{C}(36) & 1.37(1) \\ \mathrm{C}(19) & \mathrm{C}(18) & 1.34(2) & \mathrm{C}(16) & \mathrm{C}(17) & 1.41(1) \\ \mathrm{C}(37) & \mathrm{C}(36) & 1.39(1) & \mathrm{C}(29) & \mathrm{C}(30) & 1.47(1) \\ \mathrm{C}(29) & \mathrm{C}(28) & 1.55(1) & \mathrm{C}(18) & \mathrm{C}(17) & 1.33(2) \\ \mathrm{C}(21) & \mathrm{C}(25) & 1.53(1) & \mathrm{C}(21) & \mathrm{C}(24) & 1.54(1) \\ \mathrm{C}(21) & \mathrm{C}(22) & 1.52(2) & \mathrm{C}(21) & \mathrm{C}(23) & 1.53(2) \\ \mathrm{C}(25) & \mathrm{C}(26) & 1.49(2) & \mathrm{C}(25) & \mathrm{C}(28) & 1.53(1) \\ \mathrm{C}(26) & \mathrm{C}(27) & 1.58(1) & \mathrm{C}(27) & \mathrm{C}(30) & 1.53(1)\end{array}$


Table 4. Bond lengths involving hydrogens $(\AA)$

$\begin{array}{llllll}\text { atom } & \text { atom } & \text { distance } & \text { atom } & \text { atom } & \text { distance } \\ \mathrm{C}(5) & \mathrm{H}(10) & 0.950(9) & \mathrm{C}(7) & \mathrm{H}(13) & 0.95(1) \\ \mathrm{C}(7) & \mathrm{H}(14) & 0.95(1) & \mathrm{C}(14) & \mathrm{H}(20) & 0.95(1) \\ \mathrm{C}(14) & \mathrm{H}(21) & 0.95(1) & \mathrm{C}(10) & \mathrm{H}(19) & 0.95(1) \\ \mathrm{C}(9) & \mathrm{H}(17) & 0.95(1) & \mathrm{C}(9) & \mathrm{H}(18) & 0.95(1) \\ \mathrm{C}(8) & \mathrm{H}(15) & 0.95(1) & \mathrm{C}(8) & \mathrm{H}(16) & 0.95(1) \\ \mathrm{C}(34) & \mathrm{H}(46) & 0.95(1) & \mathrm{C}(34) & \mathrm{H}(47) & 0.95(1) \\ \mathrm{C}(3) & \mathrm{H}(4) & 0.95(1) & \mathrm{C}(3) & \mathrm{H}(5) & 0.95(1) \\ \mathrm{C}(3) & \mathrm{H}(6) & 0.95(2) & \mathrm{C}(40) & \mathrm{H}(52) & 0.95(1) \\ \mathrm{C}(38) & \mathrm{H}(50) & 0.95(1) & \mathrm{C}(20) & \mathrm{H}(26) & 0.95(1) \\ \mathrm{C}(19) & \mathrm{H}(25) & 0.95(1) & \mathrm{C}(16) & \mathrm{H}(22) & 0.95(1) \\ \mathrm{C}(37) & \mathrm{H}(49) & 0.95(1) & \mathrm{C}(36) & \mathrm{H}(48) & 0.95(1) \\ \mathrm{C}(39) & \mathrm{H}(51) & 0.95(2) & \mathrm{C}(4) & \mathrm{H}(7) & 0.95(1) \\ \mathrm{C}(4) & \mathrm{H}(8) & 0.95(1) & \mathrm{C}(4) & \mathrm{H}(9) & 0.95(2) \\ \mathrm{C}(6) & \mathrm{H}(11) & 0.95(1) & \mathrm{C}(6) & \mathrm{H}(12) & 0.95(1) \\ \mathrm{C}(29) & \mathrm{H}(43) & 0.95(2) & \mathrm{C}(29) & \mathrm{H}(44) & 0.95(2) \\ \mathrm{C}(2) & \mathrm{H}(1) & 0.95(1) & \mathrm{C}(2) & \mathrm{H}(2) & 0.95(1) \\ \mathrm{C}(2) & \mathrm{H}(3) & 0.95(1) & \mathrm{C}(18) & \mathrm{H}(24) & 0.95(1) \\ \mathrm{C}(25) & \mathrm{H}(36) & 0.95(1) & \mathrm{C}(26) & \mathrm{H}(37) & 0.95(2) \\ \mathrm{C}(26) & \mathrm{H}(38) & 0.95(2) & \mathrm{C}(24) & \mathrm{H}(33) & 0.95(2) \\ \mathrm{C}(24) & \mathrm{H}(34) & 0.95(2) & \mathrm{C}(24) & \mathrm{H}(35) & 0.95(1) \\ \mathrm{C}(22) & \mathrm{H}(27) & 0.95(1) & \mathrm{C}(22) & \mathrm{H}(28) & 0.95(1) \\ \mathrm{C}(22) & \mathrm{H}(29) & 0.95(2) & \mathrm{C}(27) & \mathrm{H}(39) & 0.95(2) \\ \mathrm{C}(27) & \mathrm{H}(40) & 0.95(2) & \mathrm{C}(30) & \mathrm{H}(45) & 0.95(1) \\ \mathrm{C}(17) & \mathrm{H}(23) & 0.95(2) & \mathrm{C}(28) & \mathrm{H}(41) & 0.95(2) \\ \mathrm{C}(28) & \mathrm{H}(42) & 0.95(2) & \mathrm{C}(23) & \mathrm{H}(30) & 0.95(2) \\ \mathrm{C}(23) & \mathrm{H}(31) & 0.95(2) & \mathrm{C}(23) & \mathrm{H}(32) & 0.95(2) \\ & & & & & \end{array}$


Table 5. Bond angles ( $\left.{ }^{\circ}\right)$

\begin{tabular}{|c|c|c|c|c|c|c|c|}
\hline atom & atom & atom & angle & atom & atom & atom & angle \\
\hline C(8) & C(5) & $C(1)$ & $114.2(6)$ & C(8) & C(5) & C(6) & 108.1(7) \\
\hline$C(1)$ & $C(5)$ & $\mathrm{C}(6)$ & $114.8(6)$ & $C(10)$ & $C(7)$ & $C(6)$ & $111.0(6)$ \\
\hline$C(14)$ & $C(11)$ & $C(10)$ & $112.3(5)$ & $C(14)$ & $C(11)$ & $C(13)$ & $111.0(6)$ \\
\hline$C(14)$ & $C(11)$ & $C(12)$ & $108.1(7)$ & $C(10)$ & $C(11)$ & $C(13)$ & $110.3(6)$ \\
\hline$C(10)$ & $C(11)$ & $C(12)$ & $108.5(6)$ & $C(13)$ & $C(11)$ & $C(12)$ & 6.31 \\
\hline$C(15)$ & $C(14)$ & $C(11)$ & $115.2(6)$ & C(9) & $C(10)$ & C(7) & $110.1(7)$ \\
\hline$C(9)$ & $C(10)$ & $C(11)$ & $112.2(6)$ & $C(7)$ & $C(10)$ & $C(11)$ & $111.1(5)$ \\
\hline $\mathrm{N}(2)$ & $C(13)$ & $C(11)$ & $178.8(9)$ & $C(8)$ & C(9) & $C(10)$ & $111.2(6)$ \\
\hline$C(5)$ & $C(8)$ & C(9) & $112.2(6)$ & $C(31)$ & $C(33)$ & $N(4)$ & $179.0(9)$ \\
\hline$C(31)$ & C(34) & C(35) & $115.1(7)$ & $C(20)$ & $C(15)$ & $C(16)$ & $118.4(7)$ \\
\hline$C(20)$ & $C(15)$ & $C(14)$ & $120.7(8)$ & $C(16)$ & $C(15)$ & $C(14)$ & $120.9(7)$ \\
\hline$C(4)$ & $C(1)$ & $C(2)$ & $107.1(7)$ & C(4) & $C(1)$ & $C(5)$ & $112.3(7$ \\
\hline$C(4)$ & $C(1)$ & C(3) & $109.1(7)$ & $C(2)$ & $C(1)$ & $C(5)$ & $110.2(6)$ \\
\hline$C(2)$ & $C(1)$ & $C(3)$ & $108.5(8)$ & $C(5)$ & $C(1)$ & C(3) & $109.6(6$ \\
\hline$C(35)$ & $C(40)$ & $C(39)$ & $121.4(9)$ & $C(30)$ & $C(31)$ & C(32) & $106.0(7$ \\
\hline$C(30)$ & $C(31)$ & $C(33)$ & $112.5(7)$ & $C(30)$ & $C(31)$ & C(34) & $112.6(8$ \\
\hline$C(32)$ & $C(31)$ & $C(33)$ & $106.0(9)$ & $C(32)$ & $C(31)$ & C(34) & $109.1(7$ \\
\hline$C(33)$ & $C(31)$ & $C(34)$ & $110.4(7)$ & C(37) & $C(38)$ & C(39) & $120.4(8$ \\
\hline$C(19)$ & $C(20)$ & $C(15)$ & $120.3(9)$ & $C(36)$ & $C(35)$ & C(34) & $118.9(7$ \\
\hline$C(36)$ & C(35) & $C(40)$ & $118.3(7)$ & C(34) & $C(35)$ & C(40) & $122.7(8$ \\
\hline$N(1)$ & $C(12)$ & $C(11)$ & $178(1)$ & C(18) & $C(19)$ & C(20) & $121.6(9$ \\
\hline$C(17)$ & $C(16)$ & $C(15)$ & 119.1(8) & $C(36)$ & $C(37)$ & $C(38)$ & $119.2(9)$ \\
\hline$C(35)$ & $C(36)$ & C(37) & $120.6(8)$ & $C(40)$ & $C(39)$ & $C(38)$ & $120(1)$ \\
\hline$C(5)$ & $C(6)$ & $\mathrm{C}(7)$ & $112.0(6)$ & C(30) & C(29) & C(28) & $109.6(9$ \\
\hline$C(17)$ & $C(18)$ & $C(19)$ & 119.2(9) & $C(25)$ & $C(21)$ & C(24) & $109.4(8$ \\
\hline$C(25)$ & $C(21)$ & $C(22)$ & $112.6(7)$ & C(25) & $C(21)$ & C(23) & $110.5(8$ \\
\hline$C(24)$ & $C(21)$ & $\mathrm{C}(22)$ & $108.0(8)$ & $C(24)$ & $C(21)$ & $C(23)$ & $106.8(8)$ \\
\hline$C(22)$ & $C(21)$ & $C(23)$ & $109(1)$ & $C(26)$ & $C(25)$ & C(28) & $108.4(8$ \\
\hline$C(26)$ & $C(25)$ & $C(21)$ & $113.9(8)$ & C(28) & $C(25)$ & $C(21)$ & $114.3(9$ \\
\hline$C(27)$ & $C(26)$ & $C(25)$ & 109.6(9) & $C(30)$ & $C(27)$ & $C(26)$ & $110.0(9$ \\
\hline$C(31)$ & $C(30)$ & C(29) & $113.0(8)$ & $C(31)$ & $C(30)$ & C(27) & $112.0(8)$ \\
\hline$C(29)$ & $C(30)$ & $C(27)$ & $110.5(8)$ & $C(16)$ & $C(17)$ & $C(18)$ & $121(1)$ \\
\hline $\mathrm{N}(3)$ & $C(32)$ & $C(31)$ & $174(1)$ & $\mathrm{C}(29)$ & $C(28)$ & C(25) & $111(1)$ \\
\hline
\end{tabular}


Table 6. Bond angles involving hydrogens ( $\left.{ }^{\circ}\right)$

\begin{tabular}{|c|c|c|c|c|c|c|c|}
\hline atom & atom & atom & angle & atom & atom & atom & angle \\
\hline C(8) & $\mathrm{C}(5)$ & $\mathrm{H}(10)$ & $105.2(8)$ & $C(1)$ & C(5) & $H(10)$ & $106.6(8)$ \\
\hline C(6) & $C(5)$ & $\mathrm{H}(10)$ & $107.3(7)$ & $C(10)$ & C(7) & $H(13)$ & $108.7(8)$ \\
\hline$C(10)$ & C(7) & $\mathrm{H}(14)$ & 108.7(9) & $C(6)$ & C(7) & $\mathrm{H}(13)$ & $110.0(9)$ \\
\hline$C(6)$ & $\mathrm{C}(7)$ & $\mathrm{H}(14)$ & $109.0(8)$ & $\mathrm{H}(13)$ & $C(7)$ & $\mathrm{H}(14)$ & $109.5(9)$ \\
\hline$C(15)$ & $C(14)$ & $\mathrm{H}(20)$ & 108.0(8) & C(15) & C(14) & $\mathrm{H}(21)$ & $107.7(8)$ \\
\hline $\mathrm{H}(20)$ & $C(14)$ & $\mathrm{H}(21)$ & 109.5(9) & $\mathrm{H}(20)$ & C(14) & $C(11)$ & $108.2(9)$ \\
\hline $\mathrm{H}(21)$ & $C(14)$ & $C(11)$ & 108.2(8) & $C(9)$ & $C(10)$ & $\mathrm{H}(19)$ & $107.3(7)$ \\
\hline$H(19)$ & $C(10)$ & $C(7)$ & 108.1(8) & $\mathrm{H}(19)$ & $C(10)$ & $C(11)$ & 107.8(8) \\
\hline$C(8)$ & $\mathrm{C}(9)$ & $\mathrm{H}(17)$ & 109.5(8) & $C(8)$ & $C(9)$ & $\mathrm{H}(18)$ & 108.2(9) \\
\hline$H(17)$ & $C(9)$ & $\mathrm{H}(18)$ & $109(1)$ & $\mathrm{H}(17)$ & C(9) & $C(10)$ & $109(1)$ \\
\hline$H(18)$ & $C(9)$ & $C(10)$ & $108.7(8)$ & $\mathrm{H}^{\prime}(15)$ & $C(8)$ & $H(16)$ & $109(1)$ \\
\hline$H(15)$ & $C(8)$ & $C(5)$ & 109(1) & $\mathrm{H}(15)$ & C(8) & $C(9)$ & $109.9(8)$ \\
\hline$H(16)$ & C(8) & C(5) & $107.3(9)$ & $H(16)$ & C(8) & C(9) & $107.9(9)$ \\
\hline$C(31)$ & C(34) & $\mathrm{H}(46)$ & 107.7(9) & C(31) & C(34) & $\mathrm{H}(47)$ & $108.7(7)$ \\
\hline$C(35)$ & $C(34)$ & $\mathrm{H}(46)$ & $106.3(7)$ & C(35) & C(34) & $\mathrm{H}(47)$ & 109.4(9) \\
\hline $\mathrm{H}(46)$ & $C(34)$ & $\mathrm{H}(47)$ & 109(1) & $C(1)$ & $C(3)$ & $\mathrm{H}(4)$ & $108.7(9)$ \\
\hline$C(1)$ & $C(3)$ & $H(5)$ & $109(1)$ & $C(1)$ & $C(3)$ & $\mathrm{H}(6)$ & $109(1)$ \\
\hline $\mathrm{H}(4)$ & $C(3)$ & $\mathrm{H}(5)$ & $109(1)$ & $\mathrm{H}(4)$ & $C(3)$ & $H(6)$ & $109(1)$ \\
\hline $\mathrm{H}(5)$ & C(3) & $\mathrm{H}(6)$ & $109(1)$ & C(35) & C(40) & $\mathrm{H}(52)$ & $118(1)$ \\
\hline$C(39)$ & $C(40)$ & $H(52)$ & 119(1) & $C(37)$ & $C(38)$ & $H^{\prime}(50)$ & $118(1)$ \\
\hline$C(39)$ & $C(38)$ & $\mathrm{H}(50)$ & $120(1)$ & C(19) & $C(20)$ & $\mathrm{H}(26)$ & $120(1)$ \\
\hline $\mathrm{H}(26)$ & $C(20)$ & $C(15)$ & $119.2(8)$ & C(18) & C(19) & $\mathrm{H}(25)$ & $118(1)$ \\
\hline $\mathrm{H}(25)$ & C(19) & $C(20)$ & $119(1)$ & C(17) & C(16) & $\mathrm{H}(22)$ & $120(1)$ \\
\hline $\mathrm{H}(22)$ & $C(16)$ & $C(15)$ & $120(1)$ & $C(36)$ & C(37) & $\mathrm{H}(49)$ & $120(1)$ \\
\hline $\mathrm{H}(49)$ & C(37) & $C(38)$ & $120(1)$ & $\mathrm{H}(48)$ & $C(36)$ & C(35) & $120.1(9)$ \\
\hline $\mathrm{H}(48)$ & $C(36)$ & $C(37)$ & $119(1)$ & $\mathrm{H}(51)$ & C(39) & C(40) & $120(1)$ \\
\hline $\mathrm{H}(51)$ & C(39) & $C(38)$ & $119(1)$ & $\mathrm{H}(7)$ & $C(4)$ & $H(8)$ & $109(1)$ \\
\hline $\mathrm{H}(7)$ & $\mathrm{C}(4)$ & $\mathrm{H}(9)$ & $109(1)$ & $\mathrm{H}(7)$ & $C(4)$ & $C(1)$ & $108(1)$ \\
\hline $\mathrm{H}(8)$ & C(4) & $\mathrm{H}(9)$ & 109(1) & $\mathrm{H}(8)$ & C(4) & $C(1)$ & 109(1) \\
\hline$H(9)$ & C(4) & $C(1)$ & $110.3(8)$ & $\mathrm{H}(11)$ & $C(6)$ & $\mathrm{H}(12)$ & $109(1)$ \\
\hline $\mathrm{H}(11)$ & $C(6)$ & $C(5)$ & $109.5(8)$ & $H(11)$ & $C(6)$ & $\mathrm{C}(7)$ & $110.3(9)$ \\
\hline$H(12)$ & $C(6)$ & C(5) & $108(1)$ & $H(12)$ & C(6) & C(7) & $107.3(8)$ \\
\hline$C(30)$ & C(29) & $\mathrm{H}(43)$ & $108(1)$ & $C(30)$ & C(29) & $\mathrm{H}(44)$ & $109(1)$ \\
\hline$C(28)$ & C(29) & $\mathrm{H}(43)$ & 109(1) & C(28) & C(29) & $\mathrm{H}(44)$ & 109(1) \\
\hline $\mathrm{H}(43)$ & C(29) & $\mathrm{H}(44)$ & $109(1)$ & $H(1)$ & $C(2)$ & $H(2)$ & $109(1)$ \\
\hline$H(1)$ & $C(2)$ & $\mathrm{H}(3)$ & $109(1)$ & $\mathrm{H}(1)$ & $C(2)$ & $C(1)$ & $109(1)$ \\
\hline $\mathrm{H}(2)$ & $\mathrm{C}(2)$ & $\mathrm{H}(3)$ & $109(1)$ & $\mathrm{H}(2)$ & $C(2)$ & $C(1)$ & $110(1)$ \\
\hline
\end{tabular}


Table 6. Bond angles involving hydrogens $\left({ }^{\circ}\right)$-- continued

$\begin{array}{llllllll}\text { atom } & \text { atom } & \text { atom } & \text { angle } & \text { atom } & \text { atom } & \text { atom } & \text { angle } \\ \mathrm{H}(3) & \mathrm{C}(2) & \mathrm{C}(1) & 108(1) & \mathrm{C}(17) & \mathrm{C}(18) & \mathrm{H}(24) & 119(1) \\ \mathrm{H}(24) & \mathrm{C}(18) & \mathrm{C}(19) & 120(1) & \mathrm{C}(26) & \mathrm{C}(25) & \mathrm{H}(36) & 106(1) \\ \mathrm{C}(28) & \mathrm{C}(25) & \mathrm{H}(36) & 107(1) & \mathrm{H}(36) & \mathrm{C}(25) & \mathrm{C}(21) & 105.3(9) \\ \mathrm{C}(27) & \mathrm{C}(26) & \mathrm{H}(37) & 108(1) & \mathrm{C}(27) & \mathrm{C}(26) & \mathrm{H}(38) & 110(1) \\ \mathrm{H}(37) & \mathrm{C}(26) & \mathrm{H}(38) & 109(1) & \mathrm{H}(37) & \mathrm{C}(26) & \mathrm{C}(25) & 108(1) \\ \mathrm{H}(38) & \mathrm{C}(26) & \mathrm{C}(25) & 110(1) & \mathrm{H}(33) & \mathrm{C}(24) & \mathrm{H}(34) & 109(1) \\ \mathrm{H}(33) & \mathrm{C}(24) & \mathrm{H}(35) & 109(1) & \mathrm{H}(33) & \mathrm{C}(24) & \mathrm{C}(21) & 107.1(9) \\ \mathrm{H}(34) & \mathrm{C}(24) & \mathrm{H}(35) & 109(1) & \mathrm{H}(34) & \mathrm{C}(24) & \mathrm{C}(21) & 108(1) \\ \mathrm{H}(35) & \mathrm{C}(24) & \mathrm{C}(21) & 112(1) & \mathrm{H}(27) & \mathrm{C}(22) & \mathrm{H}(28) & 109(1) \\ \mathrm{H}(27) & \mathrm{C}(22) & \mathrm{H}(29) & 109(1) & \mathrm{H}(27) & \mathrm{C}(22) & \mathrm{C}(21) & 110(1) \\ \mathrm{H}(28) & \mathrm{C}(22) & \mathrm{H}(29) & 109(1) & \mathrm{H}(28) & \mathrm{C}(22) & \mathrm{C}(21) & 108(1) \\ \mathrm{H}(29) & \mathrm{C}(22) & \mathrm{C}(21) & 109(1) & \mathrm{C}(30) & \mathrm{C}(27) & \mathrm{H}(39) & 109(1) \\ \mathrm{C}(30) & \mathrm{C}(27) & \mathrm{H}(40) & 109(1) & \mathrm{H}(39) & \mathrm{C}(27) & \mathrm{H}(40) & 109(1) \\ \mathrm{H}(39) & \mathrm{C}(27) & \mathrm{C}(26) & 110(1) & \mathrm{H}(40) & \mathrm{C}(27) & \mathrm{C}(26) & 107(1) \\ \mathrm{H}(45) & \mathrm{C}(30) & \mathrm{C}(31) & 106.8(8) & \mathrm{H}(45) & \mathrm{C}(30) & \mathrm{C}(29) & 106(1) \\ \mathrm{H}(45) & \mathrm{C}(30) & \mathrm{C}(27) & 107(1) & \mathrm{H}(23) & \mathrm{C}(17) & \mathrm{C}(16) & 119(1) \\ \mathrm{H}(23) & \mathrm{C}(17) & \mathrm{C}(18) & 119(1) & \mathrm{H}(41) & \mathrm{C}(28) & \mathrm{H}(42) & 109(1) \\ \mathrm{H}(41) & \mathrm{C}(28) & \mathrm{C}(29) & 108(1) & \mathrm{H}(41) & \mathrm{C}(28) & \mathrm{C}(25) & 105(1) \\ \mathrm{H}(42) & \mathrm{C}(28) & \mathrm{C}(29) & 109(1) & \mathrm{H}(42) & \mathrm{C}(28) & \mathrm{C}(25) & 112(1) \\ \mathrm{H}(30) & \mathrm{C}(23) & \mathrm{H}(31) & 109(1) & \mathrm{H}(30) & \mathrm{C}(23) & \mathrm{H}(32) & 109(1) \\ \mathrm{H}(30) & \mathrm{C}(23) & \mathrm{C}(21) & 110(1) & \mathrm{H}(31) & \mathrm{C}(23) & \mathrm{H}(32) & 109(1) \\ \mathrm{H}(31) & \mathrm{C}(23) & \mathrm{C}(21) & 103(1) & \mathrm{H}(32) & \mathrm{C}(23) & \mathrm{C}(21) & 114(1)\end{array}$


Table 7. Torsion Angles( $\left({ }^{\circ}\right)$

atom1 atom2 atom3 atom4 angle

$\begin{array}{lllll}C(1) & C(5) & C(8) & C(9) & -173.2(7)\end{array}$

$\mathrm{C}(1) \quad \mathrm{C}(5) \quad \mathrm{C}(8) \quad \mathrm{H}(16) \quad 68(1)$

$\begin{array}{lllll}\mathrm{C}(6) & \mathrm{C}(5) & \mathrm{C}(8) & \mathrm{H}(15) & -179.7(7)\end{array}$

$\mathrm{H}(10) \quad \mathrm{C}(5) \quad \mathrm{C}(8) \quad \mathrm{C}(9) \quad-56(1)$

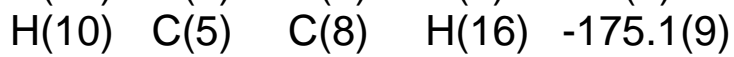

$\begin{array}{lllll}C(8) & C(5) & C(1) & C(4) & -61(1)\end{array}$

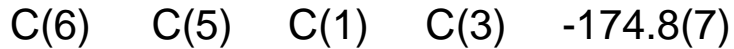

$\mathrm{C}(6) \quad \mathrm{C}(5) \quad \mathrm{C}(1) \quad \mathrm{C}(2) \quad-55.5(9)$

$\begin{array}{lllll}H(10) & \mathrm{C}(5) & \mathrm{C}(1) & \mathrm{C}(4) & -177.6(9)\end{array}$

$\begin{array}{lllll}\mathrm{C}(8) & \mathrm{C}(5) & \mathrm{C}(6) & \mathrm{C}(7) & -58.0(8)\end{array}$

$\begin{array}{lllll}\mathrm{C}(8) & \mathrm{C}(5) & \mathrm{C}(6) & \mathrm{H}(12) & -176.0(7)\end{array}$

$\begin{array}{lllll}\mathrm{C}(1) & \mathrm{C}(5) & \mathrm{C}(6) & \mathrm{H}(11) & -64(1)\end{array}$

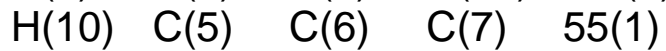

$\begin{array}{lllll}\mathrm{H}(10) & \mathrm{C}(5) & \mathrm{C}(6) & \mathrm{H}(12) & -63(1)\end{array}$

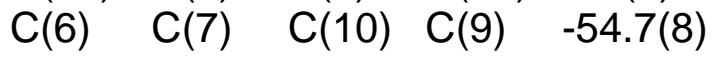

$\begin{array}{lllll}\mathrm{H}(13) & \mathrm{C}(7) & \mathrm{C}(10) & \mathrm{C}(11) & -58(1)\end{array}$

$\begin{array}{lllll}\mathrm{H}(13) & \mathrm{C}(7) & \mathrm{C}(10) & \mathrm{H}(19) & -176.7(9)\end{array}$

$\begin{array}{lllll}\mathrm{H}(14) & \mathrm{C}(7) & \mathrm{C}(10) & \mathrm{C}(9) & -174.5(7)\end{array}$

$\begin{array}{lllll}\mathrm{C}(10) & \mathrm{C}(7) & \mathrm{C}(6) & \mathrm{C}(5) & 57.8(9)\end{array}$

$\begin{array}{lllll}\mathrm{C}(10) & \mathrm{C}(7) & \mathrm{C}(6) & \mathrm{H}(12) & 176.4(9)\end{array}$

$\mathrm{H}(13) \quad \mathrm{C}(7) \quad \mathrm{C}(6) \quad \mathrm{H}(11) \quad 175(1)$

$\begin{array}{lllll}\mathrm{H}(14) & \mathrm{C}(7) & \mathrm{C}(6) & \mathrm{C}(5) & 177.5(9)\end{array}$

$\begin{array}{lllll}H(14) & \mathrm{C}(7) & \mathrm{C}(6) & \mathrm{H}(12) & -63(1)\end{array}$

C(10) $\mathrm{C}(11) \quad \mathrm{C}(14) \quad \mathrm{H}(20) \quad-60.4(8)$

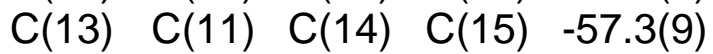

$\begin{array}{lllll}\mathrm{C}(13) & \mathrm{C}(11) & \mathrm{C}(14) & \mathrm{H}(21) & -177.8(8)\end{array}$

$\begin{array}{lllll}\mathrm{C}(12) & \mathrm{C}(11) & \mathrm{C}(14) & \mathrm{H}(20) & 179(719)\end{array}$

C(14) $\quad C(11) \quad C(10) \quad C(7) \quad-170.3(6)$

$\mathrm{C}(14) \quad \mathrm{C}(11) \quad \mathrm{C}(10) \quad \mathrm{H}(19) \quad-51(1)$

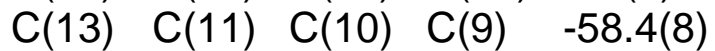

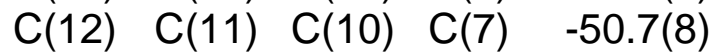

$\mathrm{C}(12) \quad \mathrm{C}(11) \quad \mathrm{C}(10) \quad \mathrm{H}(19) \quad 67.5(9)$

C(11) C(14) C(15) C(16) -85(1)

$\mathrm{H}(20) \quad \mathrm{C}(14) \quad \mathrm{C}(15) \quad \mathrm{C}(16) \quad 153.0(9)$

$\mathrm{H}(21) \quad \mathrm{C}(14) \quad \mathrm{C}(15) \quad \mathrm{C}(16) \quad 34(1)$

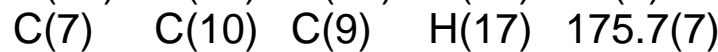

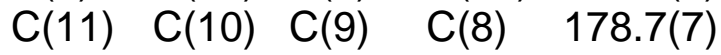

atom1 atom2 atom3 atom4 angle

$\begin{array}{lllll}\mathrm{C}(1) & \mathrm{C}(5) & \mathrm{C}(8) & \mathrm{H}(15) & -50.5(9)\end{array}$

$\begin{array}{lllll}\mathrm{C}(6) & \mathrm{C}(5) & \mathrm{C}(8) & \mathrm{C}(9) & 57.7(8)\end{array}$

$\mathrm{C}(6) \quad \mathrm{C}(5) \quad \mathrm{C}(8) \quad \mathrm{H}(16) \quad-60.7(9)$

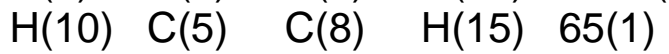

$\begin{array}{lllll}C(8) & C(5) & C(1) & C(3) & 59.5(9)\end{array}$

$\begin{array}{lllll}\mathrm{C}(8) & \mathrm{C}(5) & \mathrm{C}(1) & \mathrm{C}(2) & 178.8(7)\end{array}$

C(6) $\quad \mathrm{C}(5) \quad \mathrm{C}(1) \quad \mathrm{C}(4) \quad 63.8(9)$

$\begin{array}{lllll}H(10) & \mathrm{C}(5) & \mathrm{C}(1) & \mathrm{C}(3) & -56(1)\end{array}$

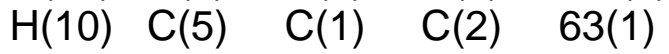

$\begin{array}{lllll}\mathrm{C}(8) & \mathrm{C}(5) & \mathrm{C}(6) & \mathrm{H}(11) & 64(1)\end{array}$

$\begin{array}{lllll}\mathrm{C}(1) & \mathrm{C}(5) & \mathrm{C}(6) & \mathrm{C}(7) & 173.3(7)\end{array}$

$\begin{array}{lllll}\mathrm{C}(1) & \mathrm{C}(5) & \mathrm{C}(6) & \mathrm{H}(12) & 55.2(9)\end{array}$

$\mathrm{H}(10) \quad \mathrm{C}(5) \quad \mathrm{C}(6) \quad \mathrm{H}(11) \quad 177(1)$

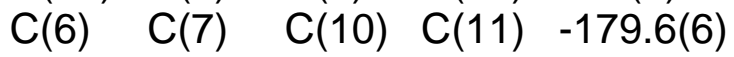

$\begin{array}{lllll}\mathrm{C}(6) & \mathrm{C}(7) & \mathrm{C}(10) & \mathrm{H}(19) & 62.2(9)\end{array}$

$\begin{array}{lllll}\mathrm{H}(13) & \mathrm{C}(7) & \mathrm{C}(10) & \mathrm{C}(9) & 66.4(9)\end{array}$

$\begin{array}{lllll}\mathrm{H}(14) & \mathrm{C}(7) & \mathrm{C}(10) & \mathrm{C}(11) & 60.5(8)\end{array}$

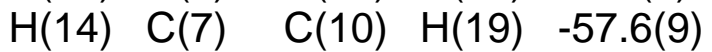

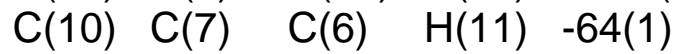

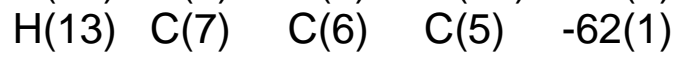

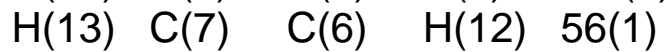

$\mathrm{H}(14) \quad \mathrm{C}(7) \quad \mathrm{C}(6) \quad \mathrm{H}(11) \quad 55(1)$

$\mathrm{C}(10) \quad \mathrm{C}(11) \quad \mathrm{C}(14) \quad \mathrm{C}(15) \quad 178.6(6)$

$\mathrm{C}(10) \quad \mathrm{C}(11) \quad \mathrm{C}(14) \quad \mathrm{H}(21) \quad 58(1)$

C(13) $\quad \mathrm{C}(11) \quad \mathrm{C}(14) \quad \mathrm{H}(20) \quad 63.7(8)$

C(12) C(11) C(14) C(15) 58.9(8)

$\mathrm{C}(12) \quad \mathrm{C}(11) \quad \mathrm{C}(14) \mathrm{H}(21) \quad-61.6(8)$

$\begin{array}{lllll}C(14) & C(11) & C(10) & C(9) & 66.0(8)\end{array}$

$\begin{array}{lllll}C(13) & C(11) & C(10) & C(7) & 65.3(8)\end{array}$

$\begin{array}{lllll}\mathrm{C}(13) & \mathrm{C}(11) & \mathrm{C}(10) & \mathrm{H}(19) & -176.4(8)\end{array}$

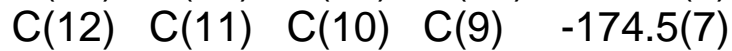

$\begin{array}{lllll}C(11) & C(14) & C(15) & C(20) & 92(1)\end{array}$

$\mathrm{H}(20) \quad \mathrm{C}(14) \quad \mathrm{C}(15) \quad \mathrm{C}(20) \quad-28(1)$

$\begin{array}{lllll}\mathrm{H}(21) & \mathrm{C}(14) & \mathrm{C}(15) & \mathrm{C}(20) & -146(1)\end{array}$

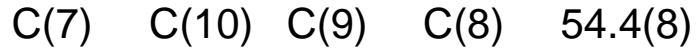

$\begin{array}{lllll}\mathrm{C}(7) \quad \mathrm{C}(10) & \mathrm{C}(9) & \mathrm{H}(18) & -64(1)\end{array}$

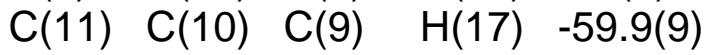


Table 7. Torsion angles (0) -- continued

atom1 atom2 atom3 atom4 angle

$\mathrm{C}(11) \quad \mathrm{C}(10) \mathrm{C}(9) \quad \mathrm{H}(18) \quad 59(1)$

$\mathrm{H}(19) \quad \mathrm{C}(10) \quad \mathrm{C}(9) \quad \mathrm{H}(17) \quad 58(1)$

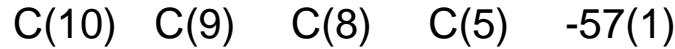

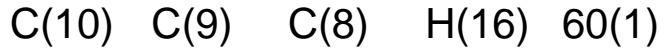

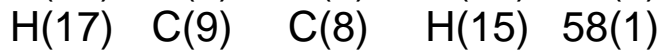

$\begin{array}{lllll}\mathrm{H}(18) & \mathrm{C}(9) & \mathrm{C}(8) & \mathrm{C}(5) & 61(1)\end{array}$

$\begin{array}{lllll}\mathrm{H}(18) & \mathrm{C}(9) & \mathrm{C}(8) & \mathrm{H}(16) & 179(881)\end{array}$

$\begin{array}{lllll}C(35) & C(34) & C(31) & C(30) & -176.2(8)\end{array}$

$\mathrm{H}(46) \quad \mathrm{C}(34) \quad \mathrm{C}(31) \quad \mathrm{C}(33) \quad 175.6(9)$

$\mathrm{H}(46) \quad \mathrm{C}(34) \quad \mathrm{C}(31) \mathrm{C}(32) \quad 59(1)$

$\mathrm{H}(47) \quad \mathrm{C}(34) \quad \mathrm{C}(31) \quad \mathrm{C}(30) \quad 60(1)$

$\mathrm{C}(31) \quad \mathrm{C}(34) \quad \mathrm{C}(35) \quad \mathrm{C}(40) \quad 87(1)$

$\mathrm{H}(46) \quad \mathrm{C}(34) \quad \mathrm{C}(35) \quad \mathrm{C}(40)-32(1)$

$\begin{array}{lllll}\mathrm{H}(47) & \mathrm{C}(34) & \mathrm{C}(35) & \mathrm{C}(40) & -150.3(9)\end{array}$

$\begin{array}{lllll}\mathrm{H}(4) & \mathrm{C}(3) & \mathrm{C}(1) & \mathrm{C}(5) & 57(1)\end{array}$

$\mathrm{H}(4) \quad \mathrm{C}(3) \quad \mathrm{C}(1) \quad \mathrm{C}(2) \quad-63(1)$

$\mathrm{H}(5) \quad \mathrm{C}(3) \quad \mathrm{C}(1) \quad \mathrm{C}(4) \quad-59(1)$

$\begin{array}{lllll}H(6) & \mathrm{C}(3) & \mathrm{C}(1) & \mathrm{C}(5) & -62(1)\end{array}$

$\begin{array}{lllll}\mathrm{H}(6) & \mathrm{C}(3) & \mathrm{C}(1) & \mathrm{C}(2) & 177.0(8)\end{array}$

$\mathrm{C}(14) \quad \mathrm{C}(15) \mathrm{C}(20) \mathrm{H}(26) \quad 0(1)$

$\mathrm{C}(16) \quad \mathrm{C}(15) \quad \mathrm{C}(20) \mathrm{H}(26) \quad 178(1)$

$\mathrm{C}(14) \quad \mathrm{C}(15) \mathrm{C}(16) \mathrm{H}(22) \quad 0(1)$

$\begin{array}{lllll}\mathrm{C}(20) & \mathrm{C}(15) & \mathrm{C}(16) & \mathrm{H}(22) & -179(1)\end{array}$

$\begin{array}{lllll}\mathrm{C}(5) & \mathrm{C}(1) & \mathrm{C}(4) & \mathrm{H}(8) & -179(1)\end{array}$

$\begin{array}{lllll}\mathrm{C}(3) & \mathrm{C}(1) & \mathrm{C}(4) & \mathrm{H}(7) & -60(1)\end{array}$

$\mathrm{C}(3) \quad \mathrm{C}(1) \quad \mathrm{C}(4) \quad \mathrm{H}(9) \quad 179(1)$

$\mathrm{C}(2) \quad \mathrm{C}(1) \quad \mathrm{C}(4) \quad \mathrm{H}(8) \quad-58(1)$

$\begin{array}{lllll}\mathrm{C}(5) & \mathrm{C}(1) & \mathrm{C}(2) & \mathrm{H}(1) & 60(1)\end{array}$

$\mathrm{C}(5) \quad \mathrm{C}(1) \quad \mathrm{C}(2) \quad \mathrm{H}(3) \quad-58(1)$

$\mathrm{C}(3) \quad \mathrm{C}(1) \quad \mathrm{C}(2) \quad \mathrm{H}(2) \quad-58(1)$

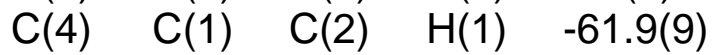

$\mathrm{C}(4) \quad \mathrm{C}(1) \quad \mathrm{C}(2) \quad \mathrm{H}(3) \quad 178(1)$

$\mathrm{C}(39) \quad \mathrm{C}(40) \quad \mathrm{C}(35) \quad \mathrm{C}(36) \quad 1(1)$

$\mathrm{H}(52) \quad \mathrm{C}(40) \quad \mathrm{C}(35) \quad \mathrm{C}(36)-178(1)$

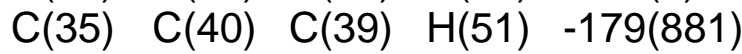

$\mathrm{H}(52) \quad \mathrm{C}(40) \quad \mathrm{C}(39) \quad \mathrm{H}(51) \quad 0(1)$

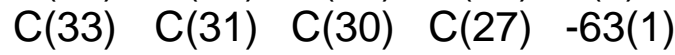

atom1 atom2 atom3 atom4 angle

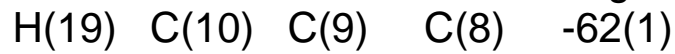

$\begin{array}{lllll}H(19) & \mathrm{C}(10) & \mathrm{C}(9) & \mathrm{H}(18) & 178(1)\end{array}$

$\begin{array}{lllll}\mathrm{C}(10) & \mathrm{C}(9) & \mathrm{C}(8) & \mathrm{H}(15) & 179(719)\end{array}$

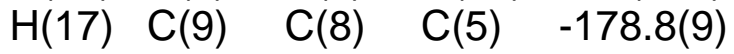

$\begin{array}{lllll}H(17) & \mathrm{C}(9) & \mathrm{C}(8) & \mathrm{H}(16) & -60(1)\end{array}$

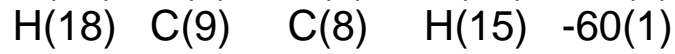

C(35) C(34) C(31) C(33) 57(1)

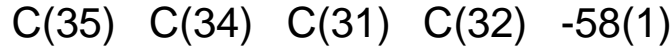

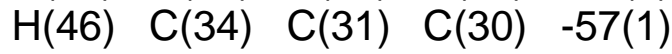

$\mathrm{H}(47) \quad \mathrm{C}(34) \quad \mathrm{C}(31) \quad \mathrm{C}(33)-65(1)$

$\mathrm{H}(47) \quad \mathrm{C}(34) \quad \mathrm{C}(31) \quad \mathrm{C}(32) \quad 178(1)$

C(31) $\quad C(34) \quad C(35) \quad C(36)-93(1)$

$\mathrm{H}(46) \quad \mathrm{C}(34) \quad \mathrm{C}(35) \quad \mathrm{C}(36) \quad 146(1)$

$\begin{array}{lllll}H(47) & \mathrm{C}(34) & \mathrm{C}(35) & \mathrm{C}(36) & 28(1)\end{array}$

$\begin{array}{lllll}\mathrm{H}(4) & \mathrm{C}(3) & \mathrm{C}(1) & \mathrm{C}(4) & -179(508)\end{array}$

$\begin{array}{lllll}\mathrm{H}(5) & \mathrm{C}(3) & \mathrm{C}(1) & \mathrm{C}(5) & 176(1)\end{array}$

$\mathrm{H}(5) \quad \mathrm{C}(3) \quad \mathrm{C}(1) \quad \mathrm{C}(2) \quad 56(1)$

$\mathrm{H}(6) \quad \mathrm{C}(3) \quad \mathrm{C}(1) \quad \mathrm{C}(4) \quad 60(1)$

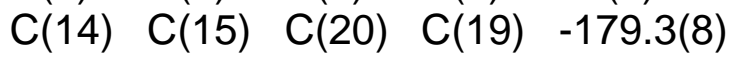

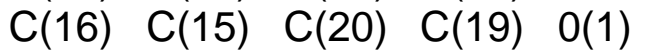

C(14) C(15) C(16) C(17) 178.3(9)

C(20) $\quad \mathrm{C}(15) \quad \mathrm{C}(16) \quad \mathrm{C}(17) \quad 0(1)$

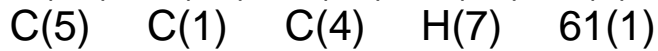

$\begin{array}{lllll}\mathrm{C}(5) & \mathrm{C}(1) & \mathrm{C}(4) & \mathrm{H}(9) & -58(1)\end{array}$

$\begin{array}{lllll}\mathrm{C}(3) & \mathrm{C}(1) & \mathrm{C}(4) & \mathrm{H}(8) & 59(1)\end{array}$

$\mathrm{C}(2) \quad \mathrm{C}(1) \quad \mathrm{C}(4) \quad \mathrm{H}(7) \quad-177(1)$

$\begin{array}{lllll}\mathrm{C}(2) & \mathrm{C}(1) & \mathrm{C}(4) & \mathrm{H}(9) & 62(1)\end{array}$

$\mathrm{C}(5) \quad \mathrm{C}(1) \quad \mathrm{C}(2) \quad \mathrm{H}(2) \quad-178(1)$

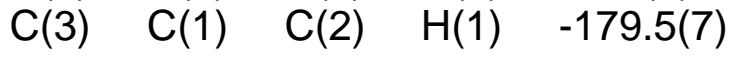

$\begin{array}{lllll}\mathrm{C}(3) & \mathrm{C}(1) & \mathrm{C}(2) & \mathrm{H}(3) & 61(1)\end{array}$

$\mathrm{C}(4) \quad \mathrm{C}(1) \quad \mathrm{C}(2) \quad \mathrm{H}(2) \quad 58(1)$

C(39) C(40) C(35) C(34) $-179(719)$

$\begin{array}{lllll}\mathrm{H}(52) & \mathrm{C}(40) & \mathrm{C}(35) & \mathrm{C}(34) & 0(1)\end{array}$

$\begin{array}{lllll}\mathrm{C}(35) & \mathrm{C}(40) & \mathrm{C}(39) & \mathrm{C}(38) & 0(1)\end{array}$

$\begin{array}{lllll}\mathrm{H}(52) & \mathrm{C}(40) & \mathrm{C}(39) & \mathrm{C}(38) & 179(359)\end{array}$

C(33) $\quad \mathrm{C}(31) \quad \mathrm{C}(30) \quad \mathrm{C}(29) \quad 61(1)$

$\mathrm{C}(33) \quad \mathrm{C}(31) \quad \mathrm{C}(30) \quad \mathrm{H}(45) \quad 178(1)$ 
Table 7. Torsion angles (0) -- continued

\begin{tabular}{|c|c|c|c|c|c|c|c|c|c|}
\hline (34) & (31) & $C(30)$ & $C(29)$ & $-63(1)$ & C(34) & $C(31)$ & $C(30)$ & C(27) & $0.5(8)$ \\
\hline (34) & (31) & $C(30)$ & $\mathrm{H}(45)$ & $52(1)$ & C(32) & $C(31)$ & $C(30)$ & $C(29)$ & \\
\hline (32) & $C(31)$ & $C(30)$ & C(27) & $51(1)$ & C(32) & $C(31)$ & $C(30)$ & $H(45)$ & $-66(1)$ \\
\hline (39) & $C(38)$ & $C(37)$ & $C(36)$ & $0(1)$ & C(39) & $C(38)$ & $C(37)$ & $\mathrm{H}(49)$ & $-179(1)$ \\
\hline$H(50)$ & $C(38)$ & $C(37)$ & $C(36)$ & $-178(1)$ & $H(50)$ & $C(38)$ & $C(37)$ & $H(49)$ & $1(1)$ \\
\hline (37) & (38) & $C(39)$ & $C(40)$ & $0(1)$ & C(37) & $C(38)$ & $C(39)$ & $H(51)$ & $178(1)$ \\
\hline (50) & $C(38)$ & $C(39)$ & $\mathrm{C}(40)$ & $178(1)$ & $H(50)$ & C(38) & C(39) & $H(51)$ & $-1(1)$ \\
\hline (15) & $C(20)$ & $C(19)$ & $C(18)$ & $0(1)$ & $C(15)$ & $C(20)$ & $C(19)$ & $H(25)$ & 179(1) \\
\hline I(26) & $C(20)$ & $C(19)$ & $C(18)$ & $-179(1)$ & $\mathrm{H}(26)$ & $C(20)$ & $C(19)$ & $H(25)$ & $0(2)$ \\
\hline$C(34)$ & $C(35)$ & $C(36)$ & C(37) & $179(623)$ & C(34) & C(35) & $C(36)$ & $\mathrm{H}(48)$ & $0(1)$ \\
\hline (40) & $C(35)$ & $C(36)$ & C(37) & $-1(1)$ & C(40) & C(35) & $C(36)$ & $H(48)$ & $178(1)$ \\
\hline C(20) & $C(19)$ & $C(18)$ & $C(17)$ & $0(1)$ & C(20) & C(19) & $C(18)$ & $\mathrm{H}(24)$ & $-179(3$ \\
\hline$H(25)$ & $C(19)$ & $C(18)$ & $C(17)$ & $-177(1)$ & $\mathrm{H}(25)$ & $C(19)$ & $C(18)$ & $\mathrm{H}(24)$ & $1(2)$ \\
\hline C(15) & $C(16)$ & $C(17)$ & $C(18)$ & 1(1) & & $C(16)$ & $C(17)$ & $H(23)$ & $179(8$ \\
\hline (22) & $C(16)$ & $C(17)$ & $C(18)$ & $-179(3$ & $\mathrm{H}(22)$ & $C(16)$ & $C(17)$ & $H(23)$ & $-1(2)$ \\
\hline$C(38)$ & $C(37)$ & $C(36)$ & $C(35)$ & $0(1)$ & C(38) & C(37) & $C(36)$ & $H(48)$ & $-179(3$ \\
\hline $\mathrm{H}(49)$ & $C(37)$ & $C(36)$ & $C(35)$ & $-179(359)$ & $\mathrm{H}(49)$ & C(37) & $C(36)$ & $\mathrm{H}(48)$ & $0(1)$ \\
\hline$C(28)$ & $C(29)$ & $C(30)$ & $C(31)$ & 175.9(9) & $C(28)$ & $C(29)$ & $C(30)$ & $C(27)$ & $-57(1)$ \\
\hline C(28) & $C(29)$ & $C(30)$ & $\mathrm{H}(45)$ & $58(1)$ & $\mathrm{H}(43)$ & C(29) & $C(30)$ & $C(31)$ & $-64(1)$ \\
\hline H(43) & C(29) & $C(30)$ & $\mathrm{C}(27)$ & $62(1)$ & $H(43)$ & C(29) & $C(30)$ & $H(45)$ & $178(1)$ \\
\hline t(44) & $C(29)$ & $C(30)$ & $C(31)$ & $55(1)$ & $\mathrm{H}(44)$ & C(29) & $C(30)$ & C(27) & $-177(1)$ \\
\hline$H(44)$ & $C(29)$ & $C(30)$ & $\mathrm{H}(45)$ & $-61(1)$ & $C(30)$ & C(29) & $C(28)$ & $C(25)$ & $59(1)$ \\
\hline C(30) & $C(29)$ & $C(28)$ & $\mathrm{H}(41)$ & & $C(30)$ & C(29) & $C(28)$ & $\mathrm{H}(42)$ & $-65(1)$ \\
\hline t(43) & C(29) & $C(28)$ & $C(25)$ & $-59(1)$ & $\mathrm{H}(43)$ & $C(29)$ & $C(28)$ & $\mathrm{H}(41)$ & $55(1)$ \\
\hline t(43) & $C(29)$ & $C(28)$ & $\mathrm{H}(42)$ & $175(1)$ & $\mathrm{H}(44)$ & $C(29)$ & $C(28)$ & $C(25)$ & $179(62$ \\
\hline$H(44)$ & $C(29)$ & $C(28)$ & $\mathrm{H}(41)$ & $-64(1)$ & $\mathrm{H}(44)$ & $C(29)$ & $C(28)$ & $\mathrm{H}(42)$ & $54(1)$ \\
\hline$C(19)$ & $C(18)$ & $C(17)$ & $C(16)$ & -1( & $C(19)$ & $C(18)$ & $C(17)$ & $\mathrm{H}(23)$ & $179(622)$ \\
\hline $\mathrm{H}(24)$ & $C(18)$ & $C(17)$ & $C(16)$ & & $\mathrm{H}(24)$ & $C(18)$ & $C(17)$ & $\mathrm{H}(23)$ & $0(2)$ \\
\hline$C(24)$ & $C(21)$ & $C(25)$ & $C(26)$ & $58(1)$ & C(24) & $C(21)$ & $C(25)$ & $C(28)$ & -175.7 \\
\hline$C(24)$ & $C(21)$ & $C(25)$ & $\mathrm{H}(36)$ & $-57(1)$ & $C(22)$ & $C(21)$ & $C(25)$ & $C(26)$ & $-61(1)$ \\
\hline$C(22)$ & $C(21)$ & $C(25)$ & $C(28)$ & 64(1) & C(22) & $C(21)$ & $C(25)$ & $H(36)$ & $-177(1)$ \\
\hline$C(23)$ & $C(21)$ & $C(25)$ & $C(26)$ & 176.2(9) & C(23) & $C(21)$ & $C(25)$ & $C(28)$ & $-58(1)$ \\
\hline$C(23)$ & $C(21)$ & $C(25)$ & $\mathrm{H}(36)$ & $59(1)$ & $C(25)$ & $C(21)$ & C(24) & $\mathrm{H}(33)$ & $-64(1)$ \\
\hline$C(25)$ & $C(21)$ & $C(24)$ & $\mathrm{H}(34)$ & $53(1)$ & $C(25)$ & $C(21)$ & $C(24)$ & $\mathrm{H}(35)$ & $175(1)$ \\
\hline$C(22)$ & $C(21)$ & $C(24)$ & $\mathrm{H}(33)$ & $58(1)$ & $C(22)$ & $C(21)$ & $C(24)$ & $\mathrm{H}(34)$ & $176(1)$ \\
\hline$C(22)$ & $C(21)$ & $C(24)$ & $\mathrm{H}(35)$ & $-62(1)$ & $C(23)$ & $C(21)$ & $C(24)$ & $\mathrm{H}(33)$ & $175(1)$ \\
\hline$C(23)$ & $C(21)$ & $C(24)$ & $\mathrm{H}(34)$ & $-66(1)$ & C(23) & $C(21)$ & $C(24)$ & $\mathrm{H}(35)$ & $55(1)$ \\
\hline
\end{tabular}


Table 7. Torsion angles (0) -- continued

\begin{tabular}{|c|c|c|c|c|c|c|c|c|c|}
\hline & $\mathrm{C}(21)$ & $\mathrm{C}(22)$ & $\mathrm{H}(27)$ & $-177(1)$ & $C(25)$ & $C(21)$ & C(22) & $\mathrm{H}(28)$ & $7(1)$ \\
\hline (25) & $C(21)$ & $C(22)$ & $H(29)$ & 61(1) & $C(24)$ & $C(21)$ & $C(22)$ & $\mathrm{H}(27)$ & $61(1)$ \\
\hline (24) & $C(21)$ & C(22) & $\mathrm{H}(28)$ & $-178(1)$ & C(24) & $C(21)$ & $C(22)$ & $\mathrm{H}(29)$ & $-59(1)$ \\
\hline$C(23)$ & $C(21)$ & $C(22)$ & $\mathrm{H}(27)$ & $-54(1)$ & $C(23)$ & $C(21)$ & $C(22)$ & $\mathrm{H}(28)$ & $65(1)$ \\
\hline (23) & $C(21)$ & $C(22)$ & $\mathrm{H}(29)$ & $-175.0(8)$ & $C(25)$ & $C(21)$ & $C(23)$ & $H(30)$ & $-173(1)$ \\
\hline (25) & $C(21)$ & $C(23)$ & $\mathrm{H}(31)$ & $-56(1)$ & $C(25)$ & $C(21)$ & $C(23)$ & $H(32)$ & $62(1)$ \\
\hline (24) & $C(21)$ & $C(23)$ & $\mathrm{H}(30)$ & $-54(1)$ & C(24) & $C(21)$ & $C(23)$ & $H(31)$ & $62(1)$ \\
\hline (24) & $C(21)$ & $C(23)$ & $\mathrm{H}(32)$ & $-178(1)$ & C(22) & $C(21)$ & $C(23)$ & $H(30)$ & $62(1)$ \\
\hline$C(22)$ & $C(21)$ & $C(23)$ & $H(31)$ & $179(1)$ & $C(22)$ & $C(21)$ & $C(23)$ & $H(32)$ & $-61(1)$ \\
\hline$C(21)$ & $C(25)$ & $C(26)$ & $C(27)$ & $-172.8(9)$ & $C(21)$ & $C(25)$ & $C(26)$ & $\mathrm{H}(37)$ & $-54(1)$ \\
\hline$C(21)$ & C(25) & $C(26)$ & $H(38)$ & $65(1)$ & C(28) & C(25) & $C(26)$ & C(27) & $58(1)$ \\
\hline$C(28)$ & C(25) & $C(26)$ & $H(37)$ & 176 & C(28) & C(25) & $C(26)$ & $H(38)$ & $-63(1)$ \\
\hline$H(36)$ & $C(25)$ & $C(26)$ & $C(27)$ & $-57(1)$ & $H(36)$ & $C(25)$ & $C(26)$ & $\mathrm{H}(37)$ & $60(1)$ \\
\hline (36) & $C(25)$ & $C(26)$ & $H(38)$ & $-179(1)$ & $C(21)$ & $C(25)$ & $C(28)$ & $C(29)$ & $172.0(9$ \\
\hline (21) & C(25) & C(28) & $H(41)$ & $54(1)$ & $C(21)$ & C(25) & C(28) & $H(42)$ & $-64(1)$ \\
\hline (26) & $C(25)$ & $C(28)$ & $C(29)$ & $-59(1)$ & $C(26)$ & $C(25)$ & $C(28)$ & $\mathrm{H}(41)$ & $-177(1)$ \\
\hline$C(26)$ & $C(25)$ & $C(28)$ & $\mathrm{H}(42)$ & $63(1)$ & $H(36)$ & $C(25)$ & $C(28)$ & $C(29)$ & $55(1)$ \\
\hline$H(36)$ & $C(25)$ & $C(28)$ & $H(41)$ & $-62(1)$ & $H(36)$ & $C(25)$ & $C(28)$ & $\mathrm{H}(42)$ & $178(1)$ \\
\hline$C(25)$ & $C(26)$ & $C(27)$ & $C(30)$ & $-58(1)$ & $C(25)$ & $C(26)$ & $C(27)$ & $H(39)$ & $62(1)$ \\
\hline C(25) & $C(26)$ & $C(27)$ & $\mathrm{H}(40)$ & $-178(1)$ & $\mathrm{H}(37)$ & C(26) & $C(27)$ & $C(30)$ & $-176(1)$ \\
\hline H(37) & $C(26)$ & $C(27)$ & $H(39)$ & $-55(1)$ & $\mathrm{H}(37)$ & C(26) & $C(27)$ & $H(40)$ & $64(1)$ \\
\hline (38) & $C(26)$ & $C(27)$ & $C(30)$ & $63(1)$ & $H(38)$ & $C(26)$ & $C(27)$ & $H(39)$ & $-175(1)$ \\
\hline (38) & $C(26)$ & $C(27)$ & $\mathrm{H}(40)$ & $-55(1)$ & $C(26)$ & C(27) & $C(30)$ & $C(31)$ & -174.8 \\
\hline$C(26)$ & $C(27)$ & $C(30)$ & C(29) & $58(1)$ & C(26) & $C(27)$ & $C(30)$ & $\mathrm{H}(45)$ & $-57(1)$ \\
\hline$H(39)$ & C(27) & $C(30)$ & $C(31)$ & $63(1)$ & $H(39)$ & C(27) & $C(30)$ & C(29) & $-63(1)$ \\
\hline$H(39)$ & $C(27)$ & $C(30)$ & $H(45)$ & $-179(719)$ & $H(40)$ & C(27) & $C(30)$ & $C(31)$ & $-56(1)$ \\
\hline $\mathrm{H}(4 \mathrm{C}$ & C(27) & $C(30)$ & C(29) & $176(1)$ & $\mathrm{H}(40)$ & C(27) & $C(30)$ & $\mathrm{H}(45)$ & $60(1)$ \\
\hline
\end{tabular}

The sign is positive if when looking from atom 2 to atom 3 a clock-wise motion of atom 1 would superimpose it on atom 4. 


\section{X-ray crystallographic Data of trans-6b}

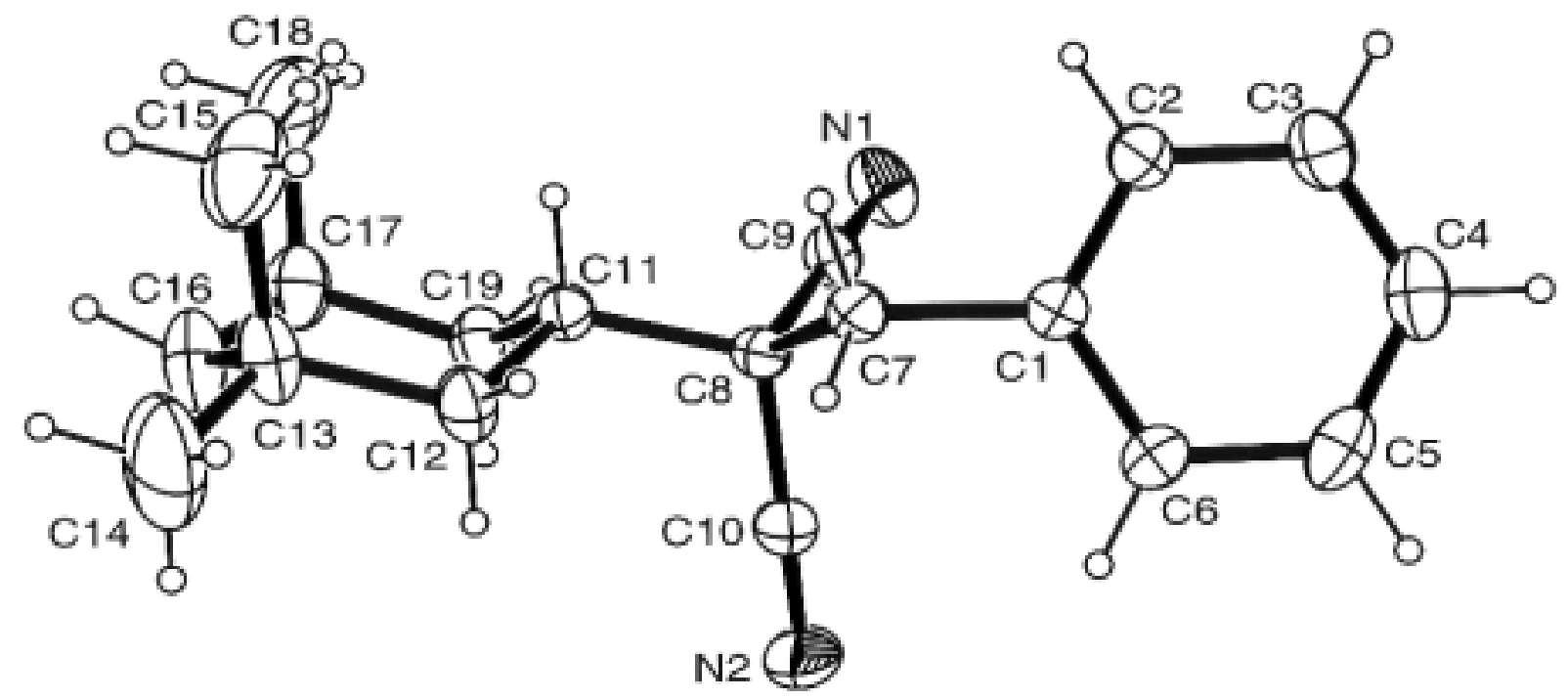

Figure S3. ORTEP drawing of trans-6b (ellipsoids at 20\% probability). 


\section{Experimental}

\section{$\underline{\text { Data Collection }}$}

A colorless prism crystal of $\mathrm{C}_{19} \mathrm{H}_{24} \mathrm{~N}_{2}$ having approximate dimensions of $0.60 \mathrm{x}$ $0.20 \times 0.10 \mathrm{~mm}$ was mounted on a glass fiber. All measurements were made on a Rigaku RAXIS RAPID imaging plate area detector with graphite monochromated Mo-Ka radiation.

Indexing was performed from 30 oscillations that were exposed for 300 seconds. The crystal-to-detector distance was $127.40 \mathrm{~mm}$.

Cell constants and an orientation matrix for data collection corresponded to a C-centered monoclinic cell with dimensions:

$$
\begin{aligned}
& \mathrm{a}=35.436(3) \AA \\
& \mathrm{b}=\quad 6.3489(5) \AA \quad \AA \quad \beta=97.655(3)^{\circ} \\
& \mathrm{c}=\quad 15.747(1) \AA \\
& \mathrm{V}=3511.3(5) \AA^{3}
\end{aligned}
$$

For $Z=8$ and F.W. $=280.41$, the calculated density is $1.06 \mathrm{~g} / \mathrm{cm}^{3}$. Based on the systematic absences of:

hkl: $\quad h+k \pm 2 n$

hOl: $\quad I \pm 2 n$

packing considerations, a statistical analysis of intensity distribution, and the successful solution and refinement of the structure, the space group was determined to be:

$\mathrm{C} 2 / \mathrm{c}(\# 15)$

The data were collected at a temperature of $23 \pm 1^{\circ} \mathrm{C}$ to a maximum $2 \theta$ value of $55.0^{\circ}$. A total of 44 oscillation images were collected. A sweep of data was done using $\omega$ scans from 130.0 to $190.0^{\circ}$ in $5.0^{\circ} \mathrm{step}$, at $\chi=45.0^{\circ}$ and $\phi=0.0^{\circ}$. The exposure rate was 600.0 [sec./0]. A second sweep was performed using $\omega$ scans from 0.0 to 160.00 in 
5.00 step, at $\chi=45.00$ and $\phi=180.00$. The exposure rate was 600.0 [sec. $/ 0$ ]. The crystal-to-detector distance was $127.40 \mathrm{~mm}$. Readout was performed in the $0.100 \mathrm{~mm}$ pixel mode.

\section{Data Reduction}

Of the 8316 reflections that were collected, 4158 were unique $\left(R_{\text {int }}=0.000\right)$; equivalent reflections were merged.

The linear absorption coefficient, $\mu$, for Mo-K $\alpha$ radiation is $0.6 \mathrm{~cm}^{-1}$. The data were corrected for Lorentz and polarization effects.

\section{$\underline{\text { Structure Solution and Refinement }}$}

The structure was solved by direct methods ${ }^{1}$ and expanded using Fourier techniques ${ }^{2}$. The non-hydrogen atoms were refined anisotropically. Hydrogen atoms were refined using the riding model. The final cycle of full-matrix least-squares refinement ${ }^{3}$ on $\mathrm{F}^{2}$ was based on 2596 observed reflections and 214 variable parameters and converged (largest parameter shift was 0.00 times its esd) with unweighted and weighted agreement factors of:

$$
\begin{gathered}
\mathrm{R} 1=\Sigma\|\mathrm{Fo}|-| \mathrm{Fc}\| / \Sigma|\mathrm{Fo}|=0.091 \\
\mathrm{wR} 2=\left[\Sigma\left(\mathrm{w}\left(\mathrm{Fo}^{2}-\mathrm{Fc}^{2}\right)^{2}\right) / \Sigma \mathrm{w}\left(\mathrm{Fo}^{2}\right)^{2}\right]^{1 / 2}=0.214
\end{gathered}
$$

The standard deviation of an observation of unit weight 4 was 0.89 . A Sheldrick weighting scheme was used. Plots of $\Sigma \mathrm{w}(|\mathrm{Fo}|-|\mathrm{Fc}|)^{2}$ versus $|\mathrm{Fo}|$, reflection order in data collection, $\sin \theta / \lambda$ and various classes of indices showed no unusual trends. The maximum and minimum peaks on the final difference Fourier map corresponded to 0.84 and $-0.43 \mathrm{e}^{-} / \AA^{3}$, respectively.

Neutral atom scattering factors were taken from Cromer and Waber 5 . Anomalous dispersion effects were included in Fcalc 6 ; the values for $\Delta f^{\prime}$ and $\Delta f^{\prime \prime}$ were those of Creagh and McAuley 7 . The values for the mass attenuation coefficients are those of Creagh and Hubbell 8 . All calculations were performed using the CrystalStructure 9,10 crystallographic software package. 
References

(1) SIR92: Altomare, A., Cascarano, G., Giacovazzo, C., Guagliardi, A., Burla, M., Polidori, G., and Camalli, M. (1994) J. Appl. Cryst., 27, 435.

(2) DIRDIF99: Beurskens, P.T., Admiraal, G., Beurskens, G., Bosman, W.P., de Gelder, R., Israel, R. and Smits, J.M.M.(1999). The DIRDIF-99 program system, Technical Report of the Crystallography Laboratory, University of Nijmegen, The Netherlands.

(3) Least Squares function minimized:

$$
\Sigma w\left(\mathrm{~F}_{\mathrm{o}}^{2}-\mathrm{F}_{\mathrm{c}}{ }^{2}\right)^{2} \quad \text { where } \mathrm{w}=\text { Least Squares weights. }
$$

(4) Standard deviation of an observation of unit weight:

$$
\begin{aligned}
& {\left[\Sigma w\left(\mathrm{~F}_{\mathrm{O}}{ }^{2}-\mathrm{F}_{\mathrm{c}}{ }^{2}\right)^{2} /\left(\mathrm{N}_{\mathrm{O}}-\mathrm{N}_{\mathrm{v}}\right)\right]^{1 / 2}} \\
& \text { where: } \quad \mathrm{N}_{\mathrm{O}}=\text { number of observations } \\
& \mathrm{N}_{\mathrm{V}}=\text { number of variables }
\end{aligned}
$$

(5) Cromer, D. T. \& Waber, J. T.; "International Tables for X-ray Crystallography", Vol. IV, The Kynoch Press, Birmingham, England, Table 2.2 A (1974).

(6) Ibers, J. A. \& Hamilton, W. C.; Acta Crystallogr., 17, 781 (1964).

(7) Creagh, D. C. \& McAuley, W.J .; "International Tables for Crystallography", Vol C, (A.J.C. Wilson, ed.), Kluwer Academic Publishers, Boston, Table 4.2.6.8, pages 219-222 (1992).

(8) Creagh, D. C. \& Hubbell, J.H..; "International Tables for Crystallography", Vol C, (A.J.C. Wilson, ed.), Kluwer Academic Publishers, Boston, Table 4.2.4.3, pages 200-206 (1992).

(9) CrystalStructure 3.5.1: Crystal Structure Analysis Package, Rigaku and Rigaku/MSC (2000-2003). 9009 New Trails Dr. The Woodlands TX 77381 USA.

(10) CRYSTALS Issue 10: Watkin, D.J., Prout, C.K. Carruthers, J.R. \& Betteridge, P.W. Chemical Crystallography Laboratory, Oxford, UK. (1996) 
EXPERIMENTAL DETAILS

\author{
A. Crystal Data
}

Empirical Formula

Formula Weight

Crystal Color, Habit

Crystal Dimensions

Crystal System

Lattice Type

Indexing Images

Detector Position

Pixel Size

Lattice Parameters

Space Group

$Z$ value

$D_{\text {calc }}$

$\mathrm{F}_{000}$
$\mathrm{C}_{19} \mathrm{H}_{24} \mathrm{~N}_{2}$

280.41

colorless, prism

$0.60 \times 0.20 \times 0.10 \mathrm{~mm}$

monoclinic

C-centered

3 oscillations @ 300.0 seconds

$127.40 \mathrm{~mm}$

$0.100 \mathrm{~mm}$

$$
\begin{aligned}
& \mathrm{a}=35.436(3) \AA \\
& \mathrm{b}=6.3489(5) \AA \\
& \mathrm{c}=15.747(1) \AA \\
& \beta=97.655(3) \circ \\
& \mathrm{V}=3511.3(5) \AA^{3}
\end{aligned}
$$

C2/c (\#15)

8

$1.061 \mathrm{~g} / \mathrm{cm}^{3}$

1216.00 
$\mu(\operatorname{MoK} \alpha)$

$0.62 \mathrm{~cm}^{-1}$ 
B. Intensity Measurements

Diffractometer

Radiation

Detector Aperture

Data Images

$\omega$ oscillation Range $(\chi=45.0, \phi=0.0)$

Exposure Rate

$\omega$ oscillation Range ( $\chi=45.0, \phi=180.0)$

Exposure Rate

Detector Position

Pixel Size

$2 \theta_{\max }$

No. of Reflections Measured

Corrections
Rigaku RAXIS-RAPID

$\operatorname{MoK} \alpha(\lambda=0.71069 \AA)$

graphite monochromated

$270 \mathrm{~mm} \times 256 \mathrm{~mm}$

44 exposures

$130.0-190.00$

$600.0 \mathrm{sec} . / 0$

$0.0-160.00$

$600.0 \mathrm{sec} . / 0$

$127.40 \mathrm{~mm}$

$0.100 \mathrm{~mm}$

$55.0^{\circ}$

Total: 8316

Unique: $4158\left(R_{\text {int }}=0.000\right)$ Lorentz-polarization 
C. Structure Solution and Refinement

Structure Solution

Refinement

Function Minimized

Least Squares Weights

Fo2)

Anomalous Dispersion

No. Observations $(\mathrm{I}>1.00 \sigma(\mathrm{I}))$

No. Variables

Reflection/Parameter Ratio

Residuals: $\mathrm{R}(\mathrm{I}>1.00 \sigma(\mathrm{I}))$

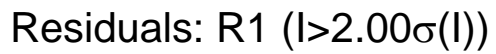

Residuals: wR2 $(\mathrm{I}>1.00 \sigma(\mathrm{I}))$

Goodness of Fit Indicator

Max Shift/Error in Final Cycle

Maximum peak in Final Diff. Map

Minimum peak in Final Diff. Map
Direct Methods (SIR92)

Full-matrix least-squares on $\mathrm{F}^{2}$

$\Sigma \mathrm{w}\left(\mathrm{Fo}^{2}-\mathrm{Fc}^{2}\right)^{2}$

$1 /\left[0.0010 \mathrm{Fo}^{2}+3.0000 \sigma\left(\mathrm{Fo}^{2}\right)+0.5000\right] /(4$

All non-hydrogen atoms

2596

214

12.13

0.102

0.091

0.214

0.885

0.000

$0.84 \mathrm{e}^{-/ \AA^{3}}$

$-0.43 \mathrm{e}^{-/} / \AA^{3}$ 
Table 1. Atomic coordinates and $\mathrm{B}_{\mathrm{iso}} / \mathrm{B}_{\text {eq }}$

$\begin{array}{llccl}\text { atom } & \mathrm{x} & \mathrm{y} & \mathrm{z} & \mathrm{B}_{\mathrm{eq}} \\ \mathrm{N}(1) & 0.3498(1) & 0.2230(8) & 0.2732(3) & 6.7(1) \\ \mathrm{N}(2) & 0.3470(1) & -0.2994(7) & 0.1107(4) & 8.2(1) \\ \mathrm{C}(1) & 0.2739(1) & 0.1677(6) & 0.0985(2) & 3.85(8) \\ \mathrm{C}(7) & 0.3103(1) & 0.1985(7) & 0.0599(2) & 4.14(8) \\ \mathrm{C}(11) & 0.3838(1) & 0.1964(7) & 0.0800(3) & 4.12(8) \\ \mathrm{C}(8) & 0.3473(1) & 0.1115(6) & 0.1134(2) & 3.93(8) \\ \mathrm{C}(15) & 0.4148(2) & 0.484(1) & -0.0593(5) & 11.9(3) \\ \mathrm{C}(2) & 0.2599(1) & 0.3296(7) & 0.1429(3) & 4.8(1) \\ \mathrm{C}(9) & 0.3483(1) & 0.1755(7) & 0.2040(3) & 4.5(1) \\ \mathrm{C}(6) & 0.2538(1) & -0.0185(7) & 0.0888(3) & 4.9(1) \\ \mathrm{C}(5) & 0.2196(1) & -0.0387(9) & 0.1236(3) & 6.2(1) \\ \mathrm{C}(4) & 0.2066(1) & 0.125(1) & 0.1676(3) & 6.3(1) \\ \mathrm{C}(10) & 0.3470(1) & -0.1221(8) & 0.1125(3) & 5.2(1) \\ \mathrm{C}(14) & 0.4217(2) & 0.175(2) & -0.1373(5) & 15.4(4) \\ \mathrm{C}(3) & 0.2264(1) & 0.3071(9) & 0.1777(3) & 5.7(1) \\ \mathrm{C}(19) & 0.4201(1) & 0.1205(9) & 0.1335(3) & 5.9(1) \\ \mathrm{C}(12) & 0.3852(1) & 0.1458(9) & -0.0139(3) & 5.8(1) \\ \mathrm{C}(17) & 0.4562(1) & 0.223(1) & 0.1065(4) & 7.1(2) \\ \mathrm{C}(13) & 0.4203(1) & 0.238(1) & -0.0474(3) & 7.2(2) \\ \mathrm{C}(16) & 0.4567(1) & 0.194(1) & 0.0122(4) & 8.9(2) \\ \mathrm{C}(18) & 0.4610(2) & 0.450(1) & 0.1403(5) & 11.0(2) \\ & & & \end{array}$

$B_{e q}=8 / 3 \pi^{2}\left(U_{11}\left(a a^{\star}\right)^{2}+U_{22}\left(b b^{\star}\right)^{2}+U_{33}\left(c c^{\star}\right)^{2}+2 U_{12}\left(a a^{*} b b^{*}\right) \cos \gamma+2 U_{13}\left(a a^{*} c c^{*}\right) \cos \beta+2 U_{23}\left(b^{*} c c^{*}\right) \cos \alpha\right)$ 
Table 2. Atomic coordinates and $B$ iso involving hydrogens $/ B_{\text {eq }}$

\begin{tabular}{|c|c|c|c|c|}
\hline atom & $X$ & $y$ & Z & $\mathrm{B}_{\mathrm{eq}}$ \\
\hline$H(1)$ & $0.2739(1)$ & $0.4576(7)$ & $0.1502(3)$ & $5.6(1)$ \\
\hline $\mathrm{H}(2)$ & $0.2166(1)$ & $0.4203(9)$ & $0.2077(3)$ & $6.8(1)$ \\
\hline $\mathrm{H}(3)$ & $0.1834(1)$ & $0.111(1)$ & $0.1913(3)$ & 7.6(2) \\
\hline $\mathrm{H}(4)$ & $0.2057(1)$ & $-0.1670(9)$ & $0.1177(3)$ & 7.3(2) \\
\hline$H(5)$ & $0.2631(1)$ & $-0.1310(7)$ & $0.0577(3)$ & $5.8(1)$ \\
\hline$H(6)$ & $0.3073(1)$ & $0.1315(7)$ & $0.0055(2)$ & $5.0(1)$ \\
\hline $\mathrm{H}(7)$ & $0.3136(1)$ & $0.3456(7)$ & $0.0527(2)$ & $5.0(1)$ \\
\hline $\mathrm{H}(8)$ & $0.3836(1)$ & $0.3455(7)$ & $0.0848(3)$ & $5.0(1)$ \\
\hline $\mathrm{H}(9)$ & $0.3863(1)$ & $-0.0030(9)$ & $-0.0193(3)$ & $7.1(2)$ \\
\hline$H(10)$ & $0.3627(1)$ & $0.1969(9)$ & $-0.0473(3)$ & $7.1(2)$ \\
\hline$H(11)$ & $0.4138(2)$ & $0.548(1)$ & $-0.0050(5)$ & $14.5(3)$ \\
\hline$H(12)$ & $0.4355(2)$ & $0.541(1)$ & $-0.0843(5)$ & 14.5(3) \\
\hline$H(13)$ & $0.3917(2)$ & $0.511(1)$ & $-0.0957(5)$ & $14.5(3)$ \\
\hline $\mathrm{H}(14)$ & $0.3985(2)$ & $0.211(2)$ & $-0.1716(5)$ & $19.0(5)$ \\
\hline$H(15)$ & $0.4424(2)$ & $0.240(2)$ & $-0.1601(5)$ & $19.0(5)$ \\
\hline$H(16)$ & $0.4249(2)$ & $0.026(2)$ & $-0.1373(5)$ & $19.0(5)$ \\
\hline $\mathrm{H}(17)$ & $0.4765(1)$ & $0.279(1)$ & $-0.0048(4)$ & $10.9(2)$ \\
\hline $\mathrm{H}(18)$ & $0.4620(1)$ & $0.050(1)$ & $0.0029(4)$ & $10.9(2)$ \\
\hline $\mathrm{H}(19)$ & $0.4775(1)$ & $0.148(1)$ & $0.1344(4)$ & $8.5(2)$ \\
\hline $\mathrm{H}(20)$ & $0.4615(2)$ & $0.453(1)$ & $0.2008(5)$ & $13.1(3)$ \\
\hline$H(21)$ & $0.4840(2)$ & $0.507(1)$ & $0.1256(5)$ & $13.1(3)$ \\
\hline $\mathrm{H}(22)$ & $0.4400(2)$ & $0.530(1)$ & $0.1140(5)$ & $13.1(3)$ \\
\hline $\mathrm{H}(23)$ & $0.4188(1)$ & $0.1495(9)$ & $0.1923(3)$ & $7.1(1)$ \\
\hline $\mathrm{H}(24)$ & $0.4215(1)$ & $-0.0274(9)$ & $0.1254(3)$ & 7.1(1) \\
\hline
\end{tabular}

$B_{e q}=8 / 3 \pi^{2}\left(U_{11}\left(a a^{\star}\right)^{2}+U_{22}\left(b b^{\star}\right)^{2}+U_{33}\left(c c c^{\star}\right)^{2}+2 U_{12}\left(a a^{*} b b^{\star}\right) \cos \gamma+2 U_{13}\left(a a^{*} c c^{\star}\right) \cos \beta+2 U_{23}\left(b^{\star} c^{\star}\right) \cos \alpha\right)$ 
Table 3. Anisotropic Displacement Parameters

$\begin{array}{lcllllr}\text { atom } & \mathrm{U}_{11} & \mathrm{U}_{22} & \mathrm{U}_{33} & \mathrm{U}_{12} & \mathrm{U}_{13} & \mathrm{U}_{23} \\ \mathrm{~N}(1) & 0.069(2) & 0.122(4) & 0.062(2) & 0.013(2) & 0.004(2) & -0.015(3) \\ \mathrm{N}(2) & 0.102(3) & 0.050(3) & 0.163(5) & 0.001(3) & 0.032(3) & 0.001(3) \\ \mathrm{C}(1) & 0.050(2) & 0.054(2) & 0.040(2) & -0.000(2) & 0.000(2) & 0.002(2) \\ \mathrm{C}(7) & 0.054(2) & 0.054(2) & 0.050(2) & -0.000(2) & 0.007(2) & 0.000(2) \\ \mathrm{C}(11) & 0.048(2) & 0.051(2) & 0.060(2) & 0.002(2) & 0.012(2) & 0.003(2) \\ \mathrm{C}(8) & 0.057(2) & 0.044(2) & 0.049(2) & 0.003(2) & 0.009(2) & -0.002(2) \\ \mathrm{C}(15) & 0.121(5) & 0.161(7) & 0.175(8) & 0.001(5) & 0.040(5) & 0.083(6) \\ \mathrm{C}(2) & 0.057(2) & 0.065(3) & 0.058(2) & 0.001(2) & 0.001(2) & -0.003(2) \\ \mathrm{C}(9) & 0.051(2) & 0.068(3) & 0.055(2) & 0.005(2) & 0.007(2) & -0.000(2) \\ \mathrm{C}(6) & 0.064(2) & 0.059(3) & 0.060(2) & -0.008(2) & 0.003(2) & 0.000(2) \\ \mathrm{C}(5) & 0.065(3) & 0.088(4) & 0.078(3) & -0.022(3) & -0.002(2) & 0.027(3) \\ \mathrm{C}(4) & 0.060(3) & 0.122(5) & 0.059(3) & 0.009(3) & 0.012(2) & 0.028(3) \\ \mathrm{C}(10) & 0.071(3) & 0.052(3) & 0.079(3) & 0.002(2) & 0.018(2) & -0.000(2) \\ \mathrm{C}(14) & 0.166(7) & 0.32(1) & 0.119(6) & -0.064(9) & 0.076(6) & -0.058(8) \\ \mathrm{C}(3) & 0.066(3) & 0.099(4) & 0.052(2) & 0.008(3) & 0.011(2) & 0.001(3) \\ \mathrm{C}(19) & 0.061(3) & 0.083(3) & 0.080(3) & 0.009(3) & 0.012(2) & 0.010(3) \\ \mathrm{C}(12) & 0.065(2) & 0.097(4) & 0.063(3) & -0.002(3) & 0.021(2) & -0.006(3) \\ \mathrm{C}(17) & 0.056(3) & 0.117(5) & 0.097(4) & 0.004(3) & 0.012(2) & 0.013(4) \\ \mathrm{C}(13) & 0.068(3) & 0.140(6) & 0.071(3) & 0.005(3) & 0.028(2) & 0.004(3) \\ \mathrm{C}(16) & 0.071(3) & 0.158(7) & 0.116(5) & 0.009(4) & 0.037(3) & 0.023(5) \\ \mathrm{C}(18) & 0.096(4) & 0.162(8) & 0.156(7) & -0.038(5) & 0.007(4) & -0.012(6)\end{array}$

The general temperature factor expression: $\exp \left(-2 \pi^{2}\left(a^{\star 2} U_{11} h^{2}+b^{\star 2} U_{22} k^{2}+\left.c^{\star 2} U_{33}\right|^{2}\right.\right.$ $\left.\left.+2 a^{*} b^{*} U_{12} h k+2 a^{*} c^{*} U_{13} h l+2 b^{*} c^{*} U_{23} k l\right)\right)$ 
Table 4. Bond lengths $(\AA ̊)$

$\begin{array}{llllll}\text { atom } & \text { atom } & \text { distance } & \text { atom } & \text { atom } & \text { distance } \\ \mathrm{N}(1) & \mathrm{C}(9) & 1.125(6) & \mathrm{N}(2) & \mathrm{C}(10) & 1.126(6) \\ \mathrm{C}(1) & \mathrm{C}(7) & 1.509(5) & \mathrm{C}(1) & \mathrm{C}(2) & 1.373(6) \\ \mathrm{C}(1) & \mathrm{C}(6) & 1.379(6) & \mathrm{C}(7) & \mathrm{C}(8) & 1.561(5) \\ \mathrm{C}(11) & \mathrm{C}(8) & 1.557(6) & \mathrm{C}(11) & \mathrm{C}(19) & 1.519(5) \\ \mathrm{C}(11) & \mathrm{C}(12) & 1.519(6) & \mathrm{C}(8) & \mathrm{C}(9) & 1.479(6) \\ \mathrm{C}(8) & \mathrm{C}(10) & 1.483(6) & \mathrm{C}(15) & \mathrm{C}(13) & 1.58(1) \\ \mathrm{C}(2) & \mathrm{C}(3) & 1.380(6) & \mathrm{C}(6) & \mathrm{C}(5) & 1.399(6) \\ \mathrm{C}(5) & \mathrm{C}(4) & 1.366(8) & \mathrm{C}(4) & \mathrm{C}(3) & 1.349(8) \\ \mathrm{C}(14) & \mathrm{C}(13) & 1.48(1) & \mathrm{C}(19) & \mathrm{C}(17) & 1.547(7) \\ \mathrm{C}(12) & \mathrm{C}(13) & 1.533(7) & \mathrm{C}(17) & \mathrm{C}(16) & 1.499(9) \\ \mathrm{C}(17) & \mathrm{C}(18) & 1.53(1) & \mathrm{C}(13) & \mathrm{C}(16) & 1.519(7)\end{array}$


Table 5. Bond lengths involving hydrogens $(\AA)$

$\begin{array}{llllll}\text { atom } & \text { atom } & \text { distance } & \text { atom } & \text { atom } & \text { distance } \\ \mathrm{C}(7) & \mathrm{H}(6) & 0.950(5) & \mathrm{C}(7) & \mathrm{H}(7) & 0.950(6) \\ \mathrm{C}(11) & \mathrm{H}(8) & 0.950(6) & \mathrm{C}(15) & \mathrm{H}(11) & 0.95(1) \\ \mathrm{C}(15) & \mathrm{H}(12) & 0.95(1) & \mathrm{C}(15) & \mathrm{H}(13) & 0.95(1) \\ \mathrm{C}(2) & \mathrm{H}(1) & 0.950(6) & \mathrm{C}(6) & \mathrm{H}(5) & 0.950(6) \\ \mathrm{C}(5) & \mathrm{H}(4) & 0.950(8) & \mathrm{C}(4) & \mathrm{H}(3) & 0.950(7) \\ \mathrm{C}(14) & \mathrm{H}(14) & 0.95(1) & \mathrm{C}(14) & \mathrm{H}(15) & 0.95(1) \\ \mathrm{C}(14) & \mathrm{H}(16) & 0.95(2) & \mathrm{C}(3) & \mathrm{H}(2) & 0.950(7) \\ \mathrm{C}(19) & \mathrm{H}(23) & 0.950(7) & \mathrm{C}(19) & \mathrm{H}(24) & 0.950(8) \\ \mathrm{C}(12) & \mathrm{H}(9) & 0.950(8) & \mathrm{C}(12) & \mathrm{H}(10) & 0.950(6) \\ \mathrm{C}(17) & \mathrm{H}(19) & 0.950(7) & \mathrm{C}(16) & \mathrm{H}(17) & 0.950(9) \\ \mathrm{C}(16) & \mathrm{H}(18) & 0.95(1) & \mathrm{C}(18) & \mathrm{H}(20) & 0.95(1) \\ \mathrm{C}(18) & \mathrm{H}(21) & 0.95(1) & \mathrm{C}(18) & \mathrm{H}(22) & 0.95(1)\end{array}$


Table 6. Bond angles (0)

$\begin{array}{llllllll}\text { atom } & \text { atom } & \text { atom } & \text { angle } & \text { atom } & \text { atom } & \text { atom } & \text { angle } \\ \mathrm{C}(7) & \mathrm{C}(1) & \mathrm{C}(2) & 119.6(4) & \mathrm{C}(7) & \mathrm{C}(1) & \mathrm{C}(6) & 121.6(4) \\ \mathrm{C}(2) & \mathrm{C}(1) & \mathrm{C}(6) & 118.8(4) & \mathrm{C}(8) & \mathrm{C}(7) & \mathrm{C}(1) & 116.0(3) \\ \mathrm{C}(8) & \mathrm{C}(11) & \mathrm{C}(19) & 112.5(3) & \mathrm{C}(8) & \mathrm{C}(11) & \mathrm{C}(12) & 113.0(3) \\ \mathrm{C}(19) & \mathrm{C}(11) & \mathrm{C}(12) & 109.8(3) & \mathrm{C}(9) & \mathrm{C}(8) & \mathrm{C}(10) & 106.4(3) \\ \mathrm{C}(9) & \mathrm{C}(8) & \mathrm{C}(7) & 109.5(3) & \mathrm{C}(9) & \mathrm{C}(8) & \mathrm{C}(11) & 108.4(3) \\ \mathrm{C}(10) & \mathrm{C}(8) & \mathrm{C}(7) & 110.2(3) & \mathrm{C}(10) & \mathrm{C}(8) & \mathrm{C}(11) & 110.3(3) \\ \mathrm{C}(7) & \mathrm{C}(8) & \mathrm{C}(11) & 111.8(3) & \mathrm{C}(3) & \mathrm{C}(2) & \mathrm{C}(1) & 120.9(4) \\ \mathrm{N}(1) & \mathrm{C}(9) & \mathrm{C}(8) & 178.8(4) & \mathrm{C}(5) & \mathrm{C}(6) & \mathrm{C}(1) & 119.8(4) \\ \mathrm{C}(4) & \mathrm{C}(5) & \mathrm{C}(6) & 119.9(5) & \mathrm{C}(3) & \mathrm{C}(4) & \mathrm{C}(5) & 120.4(5) \\ \mathrm{N}(2) & \mathrm{C}(10) & \mathrm{C}(8) & 179.0(5) & \mathrm{C}(2) & \mathrm{C}(3) & \mathrm{C}(4) & 120.3(5) \\ \mathrm{C}(17) & \mathrm{C}(19) & \mathrm{C}(11) & 112.5(4) & \mathrm{C}(13) & \mathrm{C}(12) & \mathrm{C}(11) & 112.7(4) \\ \mathrm{C}(16) & \mathrm{C}(17) & \mathrm{C}(18) & 116.5(6) & \mathrm{C}(16) & \mathrm{C}(17) & \mathrm{C}(19) & 109.9(4) \\ \mathrm{C}(18) & \mathrm{C}(17) & \mathrm{C}(19) & 110.8(5) & \mathrm{C}(16) & \mathrm{C}(13) & \mathrm{C}(15) & 109.7(5) \\ \mathrm{C}(16) & \mathrm{C}(13) & \mathrm{C}(14) & 113.9(5) & \mathrm{C}(16) & \mathrm{C}(13) & \mathrm{C}(12) & 112.2(5) \\ \mathrm{C}(15) & \mathrm{C}(13) & \mathrm{C}(14) & 100.16) & \mathrm{C}(15) & \mathrm{C}(13) & \mathrm{C}(12) & 109.1(5) \\ \mathrm{C}(14) & \mathrm{C}(13) & \mathrm{C}(12) & 111.0(5) & \mathrm{C}(17) & \mathrm{C}(16) & \mathrm{C}(13) & 117.9(5)\end{array}$


Table 7. Bond angles involving hydrogens ( $\left.{ }^{\circ}\right)$

$\begin{array}{llllllll}\text { atom } & \text { atom } & \text { atom } & \text { angle } & \text { atom } & \text { atom } & \text { atom } & \text { angle } \\ \mathrm{C}(8) & \mathrm{C}(7) & \mathrm{H}(6) & 108.1(4) & \mathrm{C}(8) & \mathrm{C}(7) & \mathrm{H}(7) & 107.7(4) \\ \mathrm{H}(6) & \mathrm{C}(7) & \mathrm{H}(7) & 109.5(5) & \mathrm{H}(6) & \mathrm{C}(7) & \mathrm{C}(1) & 107.9(4) \\ \mathrm{H}(7) & \mathrm{C}(7) & \mathrm{C}(1) & 107.5(4) & \mathrm{C}(8) & \mathrm{C}(11) & \mathrm{H}(8) & 107.6(4) \\ \mathrm{C}(19) & \mathrm{C}(11) & \mathrm{H}(8) & 106.7(4) & \mathrm{C}(12) & \mathrm{C}(11) & \mathrm{H}(8) & 106.8(5) \\ \mathrm{C}(13) & \mathrm{C}(15) & \mathrm{H}(11) & 109.4(9) & \mathrm{C}(13) & \mathrm{C}(15) & \mathrm{H}(12) & 109.7(8) \\ \mathrm{C}(13) & \mathrm{C}(15) & \mathrm{H}(13) & 109.4(8) & \mathrm{H}(11) & \mathrm{C}(15) & \mathrm{H}(12) & 109(1) \\ \mathrm{H}(11) & \mathrm{C}(15) & \mathrm{H}(13) & 109(1) & \mathrm{H}(12) & \mathrm{C}(15) & \mathrm{H}(13) & 109(1) \\ \mathrm{C}(3) & \mathrm{C}(2) & \mathrm{H}(1) & 120.1(5) & \mathrm{H}(1) & \mathrm{C}(2) & \mathrm{C}(1) & 118.9(5) \\ \mathrm{C}(5) & \mathrm{C}(6) & \mathrm{H}(5) & 120.8(5) & \mathrm{H}(5) & \mathrm{C}(6) & \mathrm{C}(1) & 119.4(5) \\ \mathrm{C}(4) & \mathrm{C}(5) & \mathrm{H}(4) & 119.8(6) & \mathrm{H}(4) & \mathrm{C}(5) & \mathrm{C}(6) & 120.3(6) \\ \mathrm{C}(3) & \mathrm{C}(4) & \mathrm{H}(3) & 120.1(7) & \mathrm{H}(3) & \mathrm{C}(4) & \mathrm{C}(5) & 119.5(7) \\ \mathrm{C}(13) & \mathrm{C}(14) & \mathrm{H}(14) & 109.8(9) & \mathrm{C}(13) & \mathrm{C}(14) & \mathrm{H}(15) & 111.7(9) \\ \mathrm{C}(13) & \mathrm{C}(14) & \mathrm{H}(16) & 106.9(9) & \mathrm{H}(14) & \mathrm{C}(14) & \mathrm{H}(15) & 109(1) \\ \mathrm{H}(14) & \mathrm{C}(14) & \mathrm{H}(16) & 109(1) & \mathrm{H}(15) & \mathrm{C}(14) & \mathrm{H}(16) & 109(1) \\ \mathrm{H}(2) & \mathrm{C}(3) & \mathrm{C}(2) & 120.5(6) & \mathrm{H}(2) & \mathrm{C}(3) & \mathrm{C}(4) & 119.2(5) \\ \mathrm{C}(17) & \mathrm{C}(19) & \mathrm{H}(23) & 109.6(5) & \mathrm{C}(17) & \mathrm{C}(19) & \mathrm{H}(24) & 108.6(5) \\ \mathrm{H}(23) & \mathrm{C}(19) & \mathrm{H}(24) & 109.5(7) & \mathrm{H}(23) & \mathrm{C}(19) & \mathrm{C}(11) & 109.4(5) \\ \mathrm{H}(24) & \mathrm{C}(19) & \mathrm{C}(11) & 107.1(4) & \mathrm{C}(13) & \mathrm{C}(12) & \mathrm{H}(9) & 107.7(5) \\ \mathrm{C}(13) & \mathrm{C}(12) & \mathrm{H}(10) & 109.6(5) & \mathrm{H}(9) & \mathrm{C}(12) & \mathrm{H}(10) & 109.4(6) \\ \mathrm{H}(9) & \mathrm{C}(12) & \mathrm{C}(11) & 107.8(5) & \mathrm{H}(10) & \mathrm{C}(12) & \mathrm{C}(11) & 109.5(5) \\ \mathrm{C}(16) & \mathrm{C}(17) & \mathrm{H}(19) & 106.4(6) & \mathrm{C}(18) & \mathrm{C}(17) & \mathrm{H}(19) & 105.6(6) \\ \mathrm{H}(19) & \mathrm{C}(17) & \mathrm{C}(19) & 107.1(6) & \mathrm{H}(17) & \mathrm{C}(16) & \mathrm{H}(18) & 109.5(8) \\ \mathrm{H}(17) & \mathrm{C}(16) & \mathrm{C}(17) & 108.4(7) & \mathrm{H}(17) & \mathrm{C}(16) & \mathrm{C}(13) & 108.7(7) \\ \mathrm{H}(18) & \mathrm{C}(16) & \mathrm{C}(17) & 107.5(7) & \mathrm{H}(18) & \mathrm{C}(16) & \mathrm{C}(13) & 104.6(6) \\ \mathrm{H}(20) & \mathrm{C}(18) & \mathrm{H}(21) & 109.5(9) & \mathrm{H}(20) & \mathrm{C}(18) & \mathrm{H}(22) & 109(1) \\ \mathrm{H}(20) & \mathrm{C}(18) & \mathrm{C}(17) & 111.0(9) & \mathrm{H}(21) & \mathrm{C}(18) & \mathrm{H}(22) & 109(1) \\ \mathrm{H}(21) & \mathrm{C}(18) & \mathrm{C}(17) & 109.3(8) & \mathrm{H}(22) & \mathrm{C}(18) & \mathrm{C}(17) & 108.1(8)\end{array}$


Table 8. Torsion Angles( $\left.{ }^{0}\right)$

atom1 atom2 atom3 atom4 angle

$\begin{array}{lllll}\mathrm{C}(2) & \mathrm{C}(1) & \mathrm{C}(7) & \mathrm{C}(8) & 98.0(4)\end{array}$

$\mathrm{C}(7) \quad \mathrm{C}(1) \quad \mathrm{C}(2) \quad \mathrm{C}(3) \quad 179.0(3)$

$\begin{array}{lllll}\mathrm{C}(7) & \mathrm{C}(1) & \mathrm{C}(6) & \mathrm{C}(5) & -178.3(4)\end{array}$

$\begin{array}{lllll}C(1) & C(7) & C(8) & C(11) & -166.4(3)\end{array}$

$\begin{array}{lllll}\mathrm{C}(1) & \mathrm{C}(7) & \mathrm{C}(8) & \mathrm{C}(10) & 70.5(5)\end{array}$

C(19) $\quad \mathrm{C}(11) \quad \mathrm{C}(8) \quad \mathrm{C}(9) \quad 56.8(4)$

$\begin{array}{lllll}C(12) & C(11) & C(8) & C(7) & -57.3(4)\end{array}$

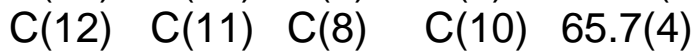

C(12) C(11) C(19) C(17) 59.3(5)

C(19) $\quad \mathrm{C}(11) \quad \mathrm{C}(12) \quad \mathrm{C}(13) \quad-56.1(5)$

$\begin{array}{lllll}\mathrm{C}(1) & \mathrm{C}(6) & \mathrm{C}(5) & \mathrm{C}(4) & -0.4(6)\end{array}$

$\begin{array}{lllll}C(5) & C(4) & C(3) & C(2) & 0.6(7)\end{array}$

C(11) C(19) C(17) C(18) 76.9(6)

C(11) C(12) C(13) C(14) 176.6(6)

C(19) C(17) C(16) C(13) 46.0(8)

C(15) C(13) C(16) C(17) 77.5(7)

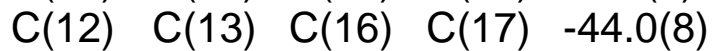

atom1 atom2 atom3 atom4 angle

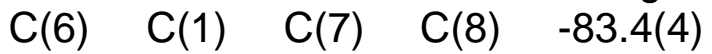

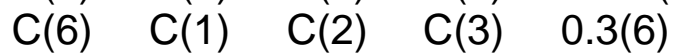

$\begin{array}{lllll}C(2) & C(1) & C(6) & C(5) & 0.2(6)\end{array}$

$\begin{array}{lllll}C(1) & C(7) & C(8) & C(9) & -46.3(5)\end{array}$

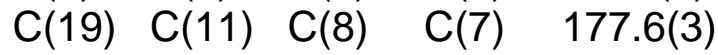

$\begin{array}{lllll}C(19) & C(11) & C(8) & C(10) & -59.4(4)\end{array}$

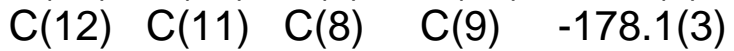

$\begin{array}{lllll}C(8) & C(11) & C(19) & C(17) & -173.9(4)\end{array}$

C(8) $\quad C(11) \quad C(12) \quad C(13) \quad 177.4(4)$

$\begin{array}{lllll}\mathrm{C}(1) & \mathrm{C}(2) & \mathrm{C}(3) & \mathrm{C}(4) & -0.8(6)\end{array}$

$\begin{array}{lllll}C(6) & C(5) & C(4) & C(3) & 0.0(7)\end{array}$

$\begin{array}{lllll}C(11) & C(19) & C(17) & C(16) & -53.2(6)\end{array}$

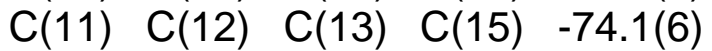

C(11) C(12) C(13) C(16) 47.7(7)

$\begin{array}{lllll}C(18) & C(17) & C(16) & C(13) & -81.1(7)\end{array}$

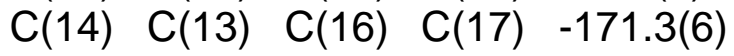

The sign is positive if when looking from atom 2 to atom 3 a clock-wise motion of atom 1 would superimpose it on atom 4. 
Table 9. Torsion Angles involving hydrogens( $\left({ }^{0}\right)$

\begin{tabular}{|c|c|c|c|c|c|c|c|c|c|}
\hline & & & & & & & & & \\
\hline (2) & $C(1)$ & $C(7)$ & $H(6)$ & $-140.6(4)$ & $C(2)$ & $C(1)$ & $\mathrm{C}(7)$ & $\mathrm{H}(7)$ & $-22.6(5)$ \\
\hline (6) & $C(1)$ & $C(7)$ & $H(6)$ & $38.0(6)$ & $C(6)$ & $C(1)$ & $\mathrm{C}(7)$ & $\mathrm{H}(7)$ & $156.0(4)$ \\
\hline (7) & $C(1)$ & $C(2)$ & $H(1)$ & $-2.0(6)$ & $C(6)$ & $C(1)$ & $C(2)$ & $H(1)$ & \\
\hline (7) & $C(1)$ & $C(6)$ & $H(5)$ & $0.9(6)$ & $C(2)$ & $C(1)$ & $C(6)$ & $H(5)$ & $179.4(4)$ \\
\hline (6) & $C(7)$ & $C(8)$ & $C(11)$ & & $H(6)$ & $\mathrm{C}(7)$ & $C(8)$ & $\mathrm{C}(9)$ & \\
\hline (6) & $C(7)$ & $C(8)$ & $C(10)$ & -50.8 & t(7) & $\mathrm{C}(7)$ & $C(8)$ & & 5) \\
\hline (7) & $C(7)$ & $C(8)$ & C(9) & $74 .{ }^{2}$ & $\mathrm{H}(7)$ & $\mathrm{C}(7)$ & $C(8)$ & $C(10)$ & \\
\hline (8) & $C(11)$ & $C(8)$ & $C(7)$ & 60.4 & $H(8)$ & $C(11)$ & $C(8)$ & $\mathrm{C}(9)$ & $-60.4(5)$ \\
\hline (8) & $C(11)$ & $C(8)$ & $C(10)$ & $-176.6(4)$ & C(8) & $C(11)$ & $C(19)$ & $\mathrm{H}(23)$ & $-51.8(6)$ \\
\hline (8 & $C(11)$ & (19) & $\mathrm{H}(24)$ & 66.8 & (12) & $C(1$ & $C(19)$ & $\mathrm{H}(23)$ & -178 \\
\hline (12) & $C(11)$ & $C(19)$ & $\mathrm{H}(24)$ & -60. & (8) & $C(11)$ & $C(1$ & $C(17)$ & -56 \\
\hline (8) & $C(11)$ & $C(19)$ & $\mathrm{H}(23)$ & 66.0( & $H(8)$ & $C(11)$ & C(19) & $H(24)$ & \\
\hline (8) & $C(11)$ & $C(12)$ & $\mathrm{H}(9)$ & -63. & $C(8)$ & $C(11)$ & $C(12)$ & $H(10)$ & 55.0 \\
\hline (19) & $C(11)$ & (12) & $H(9)$ & & & $C(1$ & & $H(10)$ & \\
\hline (8) & $C(11)$ & $C(12)$ & $C(13)$ & 59.2 & & $C(1$ & $C(12)$ & $\mathrm{H}(\mathrm{S}$ & \\
\hline (8) & $C(11)$ & $C(12)$ & $H(10)$ & $-63.1(7)$ & $H(11)$ & $C(15)$ & $C(13)$ & $C(14)$ & $-179.0(7)$ \\
\hline (11) & $C(15)$ & (13) & $C(12)$ & & 1) & $C(15)$ & $C(13)$ & & \\
\hline (12) & $C(15)$ & (13) & $C(14)$ & & & $C($ & & & \\
\hline (12) & $C(15)$ & $C(13)$ & $C(16)$ & & 3) & $C(15)$ & $C(13)$ & $C(14)$ & \\
\hline (13) & $C(15)$ & $C(13)$ & $C(12)$ & -55.5 & $H(13)$ & C(15) & $C(13)$ & $C(16)$ & $-178.8(\varepsilon$ \\
\hline (1) & $C(2)$ & $C(3)$ & $H(2)$ & -179.2( & $H(1)$ & $C(2)$ & $C(3)$ & $C(4)$ & $-179.8(5$ \\
\hline (1) & $C(2)$ & $C(3)$ & $\mathrm{H}(2)$ & & (1) & $C(6)$ & $C(5)$ & $\mathrm{H}(4)$ & \\
\hline (5) & (6) & $C(5)$ & $C(4)$ & & (5) & $C(6)$ & $C(5)$ & $\mathrm{H}(4)$ & \\
\hline (6) & $C(5)$ & $C(4)$ & $H(3)$ & -17 & $\mathrm{H}(4)$ & $C(5)$ & $C(4)$ & $C(3)$ & 178.9(5) \\
\hline (4) & $C(5)$ & $C(4)$ & $H(3)$ & $-0.9(9)$ & $C(5)$ & $\mathrm{C}(4)$ & $C(3)$ & $\mathrm{H}(2)$ & 179. \\
\hline (3) & $C(4)$ & $C(3)$ & $C(2)$ & $-179.6(5)$ & $H(3)$ & $C(4)$ & $C(3)$ & $\mathrm{H}(2)$ & $-1.1(9)$ \\
\hline $\mathrm{H}(14)$ & $C(14)$ & $C(13)$ & C(15) & & $H(14)$ & $C(14)$ & $C(13)$ & $C(12)$ & $54(1)$ \\
\hline $\mathrm{H}(14)$ & $C(14)$ & $C(13)$ & $C(16)$ & & & $C(14)$ & $C(13)$ & & \\
\hline (15) & $C(14)$ & $C(13)$ & $C(12)$ & $175(1)$ & $H(15)$ & $C(14)$ & $C(13)$ & $C(16)$ & $-56(1)$ \\
\hline$t(16)$ & $C(14)$ & $C(13)$ & $C(15)$ & $-179(359)$ & $H(16)$ & $C(14)$ & $C(13)$ & $C(12)$ & $-64.6(9)$ \\
\hline$H(16)$ & $C(14)$ & $C(13)$ & $C(16)$ & $63(1)$ & $C(11)$ & C(19) & $C(17)$ & $H(19)$ & $-168.4(6)$ \\
\hline $\mathrm{H}(23)$ & $C(19)$ & $C(17)$ & $C(16)$ & $-175.2(6)$ & $\mathrm{H}(23)$ & $C(19)$ & $C(17)$ & $C(18)$ & $-45.0(7)$ \\
\hline$t(23)$ & $C(19)$ & $C(17)$ & $H(19)$ & $69.6(8)$ & $H(24)$ & C(19) & $\mathrm{C}(17)$ & $C(16)$ & $65.3(6)$ \\
\hline $\mathrm{H}(24)$ & $C(19)$ & $C(17)$ & $C(18)$ & $-164.6(5)$ & $\mathrm{H}(24)$ & $C(19)$ & $C(17)$ & $H(19)$ & $-49.9(8)$ \\
\hline$H(9)$ & $C(12)$ & $C(13)$ & $C(15)$ & 167.2(5) & $\mathrm{H}(9)$ & $C(12)$ & $C(13)$ & $C(14)$ & $57.8(7)$ \\
\hline $\mathrm{H}(9)$ & $C(12)$ & $C(13)$ & $C(16)$ & $-71.0(6)$ & $H(10)$ & $C(12)$ & $C(13)$ & $C(15)$ & $48.2(7)$ \\
\hline H41 & $C(12)$ & $C(13)$ & $C(14)$ & $-61.2(8)$ & $H(10)$ & $C(12)$ & $C(13)$ & $C(16)$ & $170.0(6)$ \\
\hline
\end{tabular}


Table 9. Torsion angles involving hydrogens (0) -- continued

atom1 atom2 atom3 atom4 angle atom1 atom2 atom3 atom4 angle

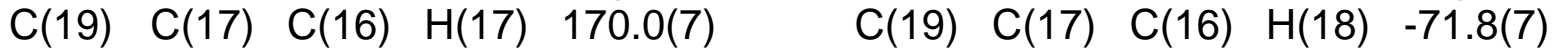

$\begin{array}{llllllll}\mathrm{C}(18) & \mathrm{C}(17) & \mathrm{C}(16) & \mathrm{H}(17) & 42.9(8) & \mathrm{C}(18) & \mathrm{C}(17) & \mathrm{C}(16) \\ \mathrm{H}(18) & 161.2(6)\end{array}$

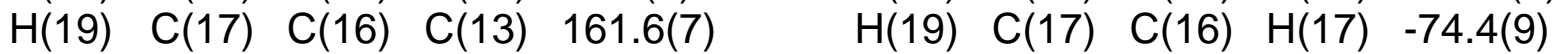

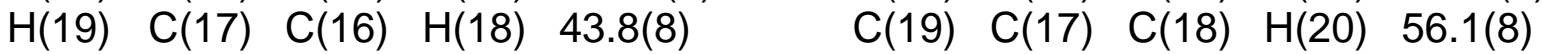

$\begin{array}{llllllll}\mathrm{C}(19) & \mathrm{C}(17) & \mathrm{C}(18) & \mathrm{H}(21) & 176.9(7) & \mathrm{C}(19) & \mathrm{C}(17) & \mathrm{C}(18) \\ \mathrm{H}(22) & -64.0(9)\end{array}$

$\begin{array}{llllllll}\mathrm{C}(16) & \mathrm{C}(17) & \mathrm{C}(18) & \mathrm{H}(20) & -177.3(6) & \mathrm{C}(16) & \mathrm{C}(17) & \mathrm{C}(18) \\ \mathrm{H}(21) & -56.5(8)\end{array}$

$\begin{array}{llllllll}\mathrm{C}(16) & \mathrm{C}(17) & \mathrm{C}(18) & \mathrm{H}(22) & 62.6(9) & \mathrm{H}(19) & \mathrm{C}(17) & \mathrm{C}(18) \\ \mathrm{H}(20) & -59.5(9)\end{array}$

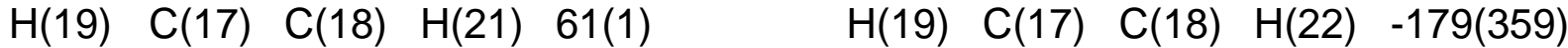

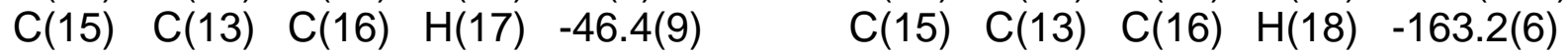

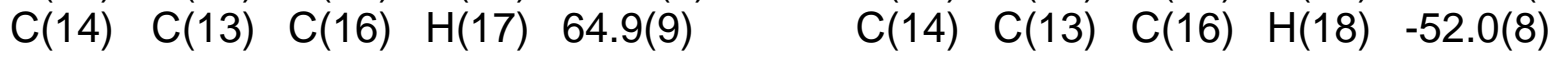

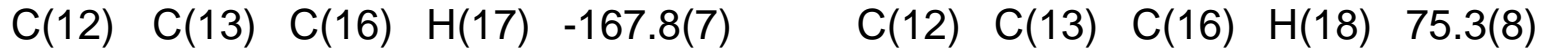

The sign is positive if when looking from atom 2 to atom 3 a clock-wise motion of atom 1 would superimpose it on atom 4 . 


\section{X-ray crystallographic Data of cis-6c}

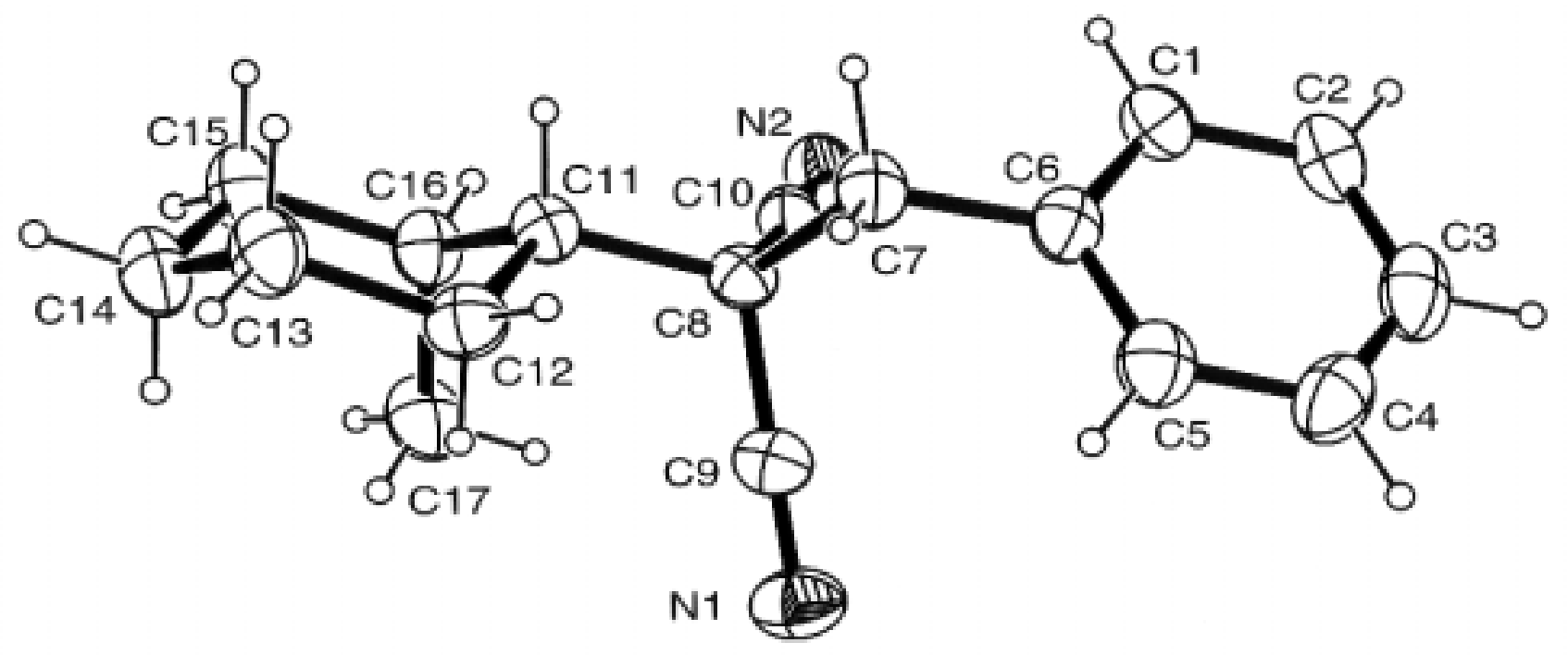

Figure S4. ORTEP drawing of cis-6c (ellipsoids at 20\% probability). 


\section{Experimental}

\section{$\underline{\text { Data Collection }}$}

A colorless block crystal of $\mathrm{C}_{17} \mathrm{H}_{20} \mathrm{~N}_{2}$ having approximate dimensions of $0.50 \mathrm{x}$ $0.25 \times 0.20 \mathrm{~mm}$ was mounted on a glass fiber. All measurements were made on a Rigaku RAXIS RAPID imaging plate area detector with graphite monochromated Mo-Ka radiation.

Indexing was performed from 30 oscillations that were exposed for 36 seconds. The crystal-to-detector distance was $127.40 \mathrm{~mm}$.

Cell constants and an orientation matrix for data collection corresponded to a primitive orthorhombic cell with dimensions:

$$
\begin{aligned}
& \mathrm{a}=10.6730(5) \AA \\
& \mathrm{b}=10.9928(7) \AA \\
& \mathrm{c}=12.8104(9) \AA \\
& \mathrm{V}=1503.0(2) \AA 3
\end{aligned}
$$

For $Z=4$ and F.W. $=252.36$, the calculated density is $1.12 \mathrm{~g} / \mathrm{cm}^{3}$. The systematic absences of:

h00: $h \pm 2 n$

$0 \mathrm{k} 0: \mathrm{k} \pm 2 \mathrm{n}$

OOl: $\quad I \pm 2 n$

uniquely determine the space group to be:

$$
\mathrm{P} 2{ }_{1} 2{ }_{1}{ }_{1}(\# 19)
$$

The data were collected at a temperature of $23 \pm 1^{\circ} \mathrm{C}$ to a maximum $2 \theta$ value of $54.0^{\circ}$. A total of 44 oscillation images were collected. A sweep of data was done using $\omega$ scans from 130.0 to $190.0^{\circ}$ in $5.0^{\circ} \mathrm{step}$, at $\chi=45.0^{\circ}$ and $\phi=0.0^{\circ}$. The exposure rate was 100.2 [sec./0]. A second sweep was performed using $\omega$ scans from 0.0 to $160.0^{\circ}$ in 
$5.0^{\circ}$ step, at $\chi=45.0^{\circ}$ and $\phi=180.0^{\circ}$. The exposure rate was 100.2 [sec. $/ 0$ ]. The crystal-to-detector distance was $127.40 \mathrm{~mm}$. Readout was performed in the $0.100 \mathrm{~mm}$ pixel mode.

\section{$\underline{\text { Data Reduction }}$}

Of the 3792 reflections that were collected, 1896 were unique $\left(R_{\text {int }}=0.000\right)$; equivalent reflections were merged.

The linear absorption coefficient, $\mu$, for Mo-K $\alpha$ radiation is $0.7 \mathrm{~cm}^{-1}$. The data were corrected for Lorentz and polarization effects.

\section{$\underline{\text { Structure Solution and Refinement }}$}

The structure was solved by direct methods ${ }^{1}$ and expanded using Fourier techniques $^{2}$. The non-hydrogen atoms were refined anisotropically. Hydrogen atoms were refined using the riding model. The final cycle of full-matrix least-squares refinement ${ }^{3}$ on $\mathrm{F}^{2}$ was based on 1878 observed reflections and 192 variable parameters and converged (largest parameter shift was 0.00 times its esd) with unweighted and weighted agreement factors of:

$$
\begin{gathered}
\mathrm{R} 1=\Sigma\|\mathrm{Fo}|-| \mathrm{Fc}\| / \Sigma|\mathrm{Fo}|=0.061 \\
\mathrm{wR} 2=\left[\Sigma\left(\mathrm{w}\left(\mathrm{Fo}^{2}-\mathrm{Fc}^{2}\right)^{2}\right) / \Sigma \mathrm{w}\left(\mathrm{Fo}^{2}\right)^{2}\right]^{1 / 2}=0.185
\end{gathered}
$$

The standard deviation of an observation of unit weight 4 was 0.91 . A Sheldrick weighting scheme was used. Plots of $\Sigma \mathrm{w}(|\mathrm{Fo}|-|\mathrm{Fc}|)^{2}$ versus $|\mathrm{Fo}|$, reflection order in data collection, $\sin \theta / \lambda$ and various classes of indices showed no unusual trends. The maximum and minimum peaks on the final difference Fourier map corresponded to 0.36 and $-0.32 \mathrm{e}^{-} / \AA^{3}$, respectively.

Neutral atom scattering factors were taken from Cromer and Waber 5 . Anomalous dispersion effects were included in Fcalc 6 ; the values for $\Delta f^{\prime}$ and $\Delta f^{\prime \prime}$ were those of Creagh and McAuley 7 . The values for the mass attenuation coefficients are those of Creagh and Hubbell 8 . All calculations were performed using the CrystalStructure 9,10 crystallographic software package. 
References

(1) SIR92: Altomare, A., Cascarano, G., Giacovazzo, C., Guagliardi, A., Burla, M., Polidori, G., and Camalli, M. (1994) J. Appl. Cryst., 27, 435.

(2) DIRDIF99: Beurskens, P.T., Admiraal, G., Beurskens, G., Bosman, W.P., de Gelder, R., Israel, R. and Smits, J.M.M.(1999). The DIRDIF-99 program system, Technical Report of the Crystallography Laboratory, University of Nijmegen, The Netherlands.

(3) Least Squares function minimized:

$$
\Sigma w\left(\mathrm{~F}_{\mathrm{o}}^{2}-\mathrm{F}_{\mathrm{c}}{ }^{2}\right)^{2} \quad \text { where } \mathrm{w}=\text { Least Squares weights. }
$$

(4) Standard deviation of an observation of unit weight:

$$
\begin{aligned}
& {\left[\Sigma w\left(\mathrm{~F}_{\mathrm{O}}{ }^{2}-\mathrm{F}_{\mathrm{c}}{ }^{2}\right)^{2} /\left(\mathrm{N}_{\mathrm{O}}-\mathrm{N}_{\mathrm{v}}\right)\right]^{1 / 2}} \\
& \text { where: } \quad \mathrm{N}_{\mathrm{O}}=\text { number of observations } \\
& \mathrm{N}_{\mathrm{V}}=\text { number of variables }
\end{aligned}
$$

(5) Cromer, D. T. \& Waber, J. T.; "International Tables for X-ray Crystallography", Vol. IV, The Kynoch Press, Birmingham, England, Table 2.2 A (1974).

(6) Ibers, J. A. \& Hamilton, W. C.; Acta Crystallogr., 17, 781 (1964).

(7) Creagh, D. C. \& McAuley, W.J .; "International Tables for Crystallography", Vol C, (A.J.C. Wilson, ed.), Kluwer Academic Publishers, Boston, Table 4.2.6.8, pages 219-222 (1992).

(8) Creagh, D. C. \& Hubbell, J.H..; "International Tables for Crystallography", Vol C, (A.J.C. Wilson, ed.), Kluwer Academic Publishers, Boston, Table 4.2.4.3, pages 200-206 (1992).

(9) CrystalStructure 3.5.1: Crystal Structure Analysis Package, Rigaku and Rigaku/MSC (2000-2003). 9009 New Trails Dr. The Woodlands TX 77381 USA.

(10) CRYSTALS Issue 10: Watkin, D.J., Prout, C.K. Carruthers, J.R. \& Betteridge, P.W. Chemical Crystallography Laboratory, Oxford, UK. (1996) 
EXPERIMENTAL DETAILS

\author{
A. Crystal Data
}

Empirical Formula

Formula Weight

Crystal Color, Habit

Crystal Dimensions

Crystal System

Lattice Type

Indexing Images

Detector Position

Pixel Size

Lattice Parameters

Space Group

$Z$ value

$D_{\text {calc }}$

$\mathrm{F}_{000}$

$\mu(\operatorname{MoK} \alpha)$
$\mathrm{C}_{17} \mathrm{H}_{20} \mathrm{~N}_{2}$

252.36

colorless, block

$0.50 \times 0.25 \times 0.20 \mathrm{~mm}$

orthorhombic

Primitive

3 oscillations @ 36.0 seconds

$127.40 \mathrm{~mm}$

$0.100 \mathrm{~mm}$

$$
\begin{aligned}
& \mathrm{a}=10.6730(5) \AA \\
& \mathrm{b}=10.9928(7) \AA \\
& \mathrm{c}=12.8104(9) \AA \\
& \mathrm{V}=1503.0(2) \AA^{3}
\end{aligned}
$$

$\mathrm{P} 2{ }_{1} 2{ }_{1}{ }_{1}(\# 19)$

4

$1.115 \mathrm{~g} / \mathrm{cm}^{3}$

544.00

$0.66 \mathrm{~cm}^{-1}$ 
B. Intensity Measurements

Diffractometer

Radiation

Detector Aperture

Data Images

$\omega$ oscillation Range $(\chi=45.0, \phi=0.0)$

Exposure Rate

$\omega$ oscillation Range ( $\chi=45.0, \phi=180.0)$

Exposure Rate

Detector Position

Pixel Size

$2 \theta_{\max }$

No. of Reflections Measured

Corrections
Rigaku RAXIS-RAPID

$\operatorname{MoK} \alpha(\lambda=0.71069 \AA)$

graphite monochromated

$270 \mathrm{~mm} \times 256 \mathrm{~mm}$

44 exposures

$130.0-190.00$

$100.2 \mathrm{sec} . / \mathrm{O}$

$0.0-160.00$

$100.2 \mathrm{sec} . / \mathrm{O}$

$127.40 \mathrm{~mm}$

$0.100 \mathrm{~mm}$

$54.0^{0}$

Total: 3792

Unique: $1896\left(R_{\text {int }}=0.000\right)$ Lorentz-polarization 
C. Structure Solution and Refinement

Structure Solution

Refinement

Function Minimized

Least Squares Weights

Fo2)

Anomalous Dispersion

No. Observations (All reflections)

No. Variables

Reflection/Parameter Ratio

Residuals: $\mathrm{R}$ (All reflections)

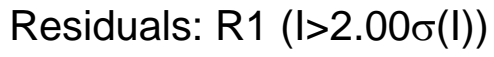

Residuals: wR2 (All reflections)

Goodness of Fit Indicator

Max Shift/Error in Final Cycle

Maximum peak in Final Diff. Map

Minimum peak in Final Diff. Map
Direct Methods (SIR92)

Full-matrix least-squares on $\mathrm{F}^{2}$

$\Sigma \mathrm{w}\left(\mathrm{Fo}^{2}-\mathrm{Fc}^{2}\right)^{2}$

$1 /\left[0.0010 \mathrm{Fo}^{2}+0.4000 \sigma\left(\mathrm{Fo}^{2}\right)+0.0400\right] /(4$

All non-hydrogen atoms

1878

192

9.78

0.111

0.061

0.185

0.908

0.000

$0.36 \mathrm{e}^{-/ A^{3}}$

$-0.32 e^{-} / \AA^{3}$ 
Table 1. Atomic coordinates and $\mathrm{B}_{\mathrm{iso}} / \mathrm{B}_{\text {eq }}$

$\begin{array}{lcccc}\text { atom } & \mathrm{x} & \mathrm{y} & \mathrm{z} & \mathrm{B}_{\text {eq }} \\ \mathrm{C}(10) & 0.1153(5) & 0.1380(5) & 0.2666(3) & 5.5(1) \\ \mathrm{N}(2) & 0.0404(4) & 0.0792(4) & 0.2273(3) & 6.9(1) \\ \mathrm{N}(1) & 0.4345(4) & 0.1334(5) & 0.2754(4) & 8.0(1) \\ \mathrm{C}(8) & 0.2092(4) & 0.2159(4) & 0.3192(3) & 5.1(1) \\ \mathrm{C}(11) & 0.1822(5) & 0.2183(4) & 0.4413(3) & 5.4(1) \\ \mathrm{C}(6) & 0.2205(5) & 0.3563(4) & 0.1540(4) & 5.9(1) \\ \mathrm{C}(9) & 0.3354(5) & 0.1657(4) & 0.2943(4) & 5.9(1) \\ \mathrm{C}(12) & 0.2910(5) & 0.2744(5) & 0.5024(4) & 6.6(1) \\ \mathrm{C}(13) & 0.2548(6) & 0.2903(5) & 0.6193(4) & 7.5(2) \\ \mathrm{C}(7) & 0.1994(6) & 0.3480(4) & 0.2714(3) & 6.3(1) \\ \mathrm{C}(1) & 0.1220(5) & 0.3433(5) & 0.0851(4) & 6.8(1) \\ \mathrm{C}(2) & 0.1427(7) & 0.3481(5) & -0.0236(4) & 7.5(2) \\ \mathrm{C}(17) & 0.2348(7) & -0.0090(5) & 0.4734(4) & 8.4(2) \\ \mathrm{C}(14) & 0.2162(6) & 0.1708(5) & 0.6674(4) & 7.7(2) \\ \mathrm{C}(3) & 0.2612(8) & 0.3685(5) & -0.0606(5) & 8.3(2) \\ \mathrm{C}(15) & 0.1118(6) & 0.1133(5) & 0.6057(4) & 7.5(1) \\ \mathrm{C}(16) & 0.1413(5) & 0.0944(5) & 0.4891(4) & 6.2(1) \\ \mathrm{C}(4) & 0.3599(7) & 0.3832(5) & 0.0062(5) & 8.3(2) \\ \mathrm{C}(5) & 0.3383(6) & 0.3767(5) & 0.1134(5) & 7.2(1)\end{array}$

$B_{\text {eq }}=8 / 3 \pi^{2}\left(U_{11}\left(a^{*}\right)^{2}+U_{22}\left(b^{*}\right)^{2}+U_{33}\left(\left(c^{*}\right)^{2}+2 U_{12}\left(a a^{*} b b^{*}\right) \cos \gamma+2 U_{13}\left(a a^{*} c c^{*}\right) \cos \beta+2 U_{23}\left(b b^{*} c c^{*}\right) \cos \alpha\right)\right.$ 
Table 2. Atomic coordinates and $B$ iso involving hydrogens $/ B_{\text {eq }}$

\begin{tabular}{|c|c|c|c|c|}
\hline atom & $x$ & $y$ & $z$ & $\mathrm{~B}_{\mathrm{eq}}$ \\
\hline$H(1)$ & $0.0403(5)$ & $0.3318(5)$ & $0.1129(4)$ & $8.1(2)$ \\
\hline $\mathrm{H}(2)$ & $0.0760(7)$ & $0.3390(5)$ & $-0.0721(4)$ & $9.0(2)$ \\
\hline $\mathrm{H}(3)$ & $0.2739(8)$ & $0.3711(5)$ & $-0.1340(5)$ & $9.9(2)$ \\
\hline $\mathrm{H}(4)$ & $0.4404(7)$ & $0.3975(5)$ & $-0.0231(5)$ & $9.9(2)$ \\
\hline$H(5)$ & $0.4070(6)$ & $0.3838(5)$ & $0.1601(5)$ & $8.6(2)$ \\
\hline$H(6)$ & $0.1179(6)$ & $0.3784(4)$ & $0.2857(3)$ & $7.5(2)$ \\
\hline $\mathrm{H}(7)$ & $0.2599(6)$ & $0.3979(4)$ & $0.3049(3)$ & 7.5(2) \\
\hline $\mathrm{H}(8)$ & $0.1144(5)$ & $0.2731(4)$ & $0.4509(3)$ & 6.4(1) \\
\hline$H(9)$ & $0.3594(5)$ & $0.2194(5)$ & $0.4976(4)$ & 7.9(2) \\
\hline $\mathrm{H}(10)$ & $0.3136(5)$ & $0.3498(5)$ & $0.4716(4)$ & 7.9(2) \\
\hline$H(11)$ & $0.3221(6)$ & $0.3250(5)$ & $0.6575(4)$ & $9.0(2)$ \\
\hline $\mathrm{H}(12)$ & $0.1842(6)$ & $0.3428(5)$ & $0.6227(4)$ & $9.0(2)$ \\
\hline$H(13)$ & $0.2857(6)$ & $0.1175(5)$ & $0.6600(4)$ & $9.3(2)$ \\
\hline $\mathrm{H}(14)$ & $0.1970(6)$ & $0.1802(5)$ & $0.7394(4)$ & $9.3(2)$ \\
\hline $\mathrm{H}(15)$ & $0.0913(6)$ & $0.0376(5)$ & $0.6371(4)$ & $9.1(2)$ \\
\hline $\mathrm{H}(16)$ & $0.0428(6)$ & $0.1674(5)$ & $0.6120(4)$ & $9.1(2)$ \\
\hline $\mathrm{H}(17)$ & $0.0669(5)$ & $0.0691(5)$ & $0.4547(4)$ & $7.5(2)$ \\
\hline $\mathrm{H}(18)$ & $0.2049(7)$ & $-0.0824(5)$ & $0.5037(4)$ & $10.1(2)$ \\
\hline $\mathrm{H}(19)$ & $0.2518(7)$ & $-0.0219(5)$ & $0.4015(4)$ & $10.1(2)$ \\
\hline $\mathrm{H}(20)$ & $0.3094(7)$ & $0.0156(5)$ & $0.5079(4)$ & $10.1(2)$ \\
\hline
\end{tabular}


Table 3. Anisotropic Displacement Parameters

$\begin{array}{lcccccc}\text { atom } & \mathrm{U}_{11} & \mathrm{U}_{22} & \mathrm{U}_{33} & \mathrm{U}_{12} & \mathrm{U}_{13} & \mathrm{U}_{23} \\ \mathrm{C}(10) & 0.083(3) & 0.066(3) & 0.061(2) & 0.010(3) & 0.005(3) & 0.004(2) \\ \mathrm{N}(2) & 0.088(3) & 0.090(3) & 0.085(3) & -0.011(3) & -0.009(2) & 0.002(3) \\ \mathrm{N}(1) & 0.085(3) & 0.102(3) & 0.118(4) & 0.014(3) & 0.001(3) & -0.030(3) \\ \mathrm{C}(8) & 0.071(3) & 0.053(2) & 0.069(3) & 0.005(2) & -0.003(2) & -0.005(2) \\ \mathrm{C}(11) & 0.077(3) & 0.059(2) & 0.068(3) & 0.001(2) & -0.004(2) & -0.002(2) \\ \mathrm{C}(6) & 0.096(3) & 0.052(2) & 0.075(3) & 0.001(3) & 0.009(3) & 0.003(2) \\ \mathrm{C}(9) & 0.073(3) & 0.064(3) & 0.086(3) & 0.003(3) & -0.009(3) & -0.013(3) \\ \mathrm{C}(12) & 0.080(3) & 0.081(3) & 0.089(3) & 0.000(3) & 0.001(3) & -0.008(3) \\ \mathrm{C}(13) & 0.114(4) & 0.096(4) & 0.075(3) & -0.004(4) & -0.015(3) & -0.015(3) \\ \mathrm{C}(7) & 0.110(4) & 0.056(2) & 0.072(3) & 0.010(3) & 0.003(3) & -0.002(2) \\ \mathrm{C}(1) & 0.095(4) & 0.075(3) & 0.088(3) & 0.008(3) & -0.002(3) & 0.015(3) \\ \mathrm{C}(2) & 0.124(5) & 0.079(4) & 0.082(4) & -0.005(4) & -0.019(4) & 0.016(3) \\ \mathrm{C}(17) & 0.157(5) & 0.063(3) & 0.099(4) & 0.028(4) & -0.009(4) & 0.001(3) \\ \mathrm{C}(14) & 0.130(5) & 0.092(4) & 0.071(3) & 0.006(4) & -0.016(3) & 0.001(3) \\ \mathrm{C}(3) & 0.171(7) & 0.061(3) & 0.081(4) & 0.009(4) & 0.021(4) & 0.009(3) \\ \mathrm{C}(15) & 0.128(4) & 0.079(3) & 0.080(3) & -0.007(4) & -0.007(4) & 0.010(3) \\ \mathrm{C}(16) & 0.098(4) & 0.066(3) & 0.072(3) & -0.006(3) & -0.002(3) & 0.002(2) \\ \mathrm{C}(4) & 0.125(5) & 0.086(4) & 0.105(4) & -0.004(4) & 0.034(4) & 0.009(4) \\ \mathrm{C}(5) & 0.108(4) & 0.075(3) & 0.090(4) & -0.005(3) & 0.002(3) & 0.005(3)\end{array}$

The general temperature factor expression: $\exp \left(-2 \pi^{2}\left(a^{*} U_{11} h^{2}+b^{\star 2} U_{22} k^{2}+\left.c^{\star 2} U_{33}\right|^{2}\right.\right.$ $\left.\left.+2 a^{*} b^{*} U_{12} h k+2 a^{*} c^{*} U_{13} h l+2 b^{*} c^{*} U_{23} k l\right)\right)$ 
Table 4. Bond lengths $(\AA ̊)$

$\begin{array}{llllll}\text { atom } & \text { atom } & \text { distance } & \text { atom } & \text { atom } & \text { distance } \\ \mathrm{C}(10) & \mathrm{N}(2) & 1.145(7) & \mathrm{C}(10) & \mathrm{C}(8) & 1.481(7) \\ \mathrm{N}(1) & \mathrm{C}(9) & 1.141(7) & \mathrm{C}(8) & \mathrm{C}(11) & 1.590(6) \\ \mathrm{C}(8) & \mathrm{C}(9) & 1.491(7) & \mathrm{C}(8) & \mathrm{C}(7) & 1.579(6) \\ \mathrm{C}(11) & \mathrm{C}(12) & 1.530(7) & \mathrm{C}(11) & \mathrm{C}(16) & 1.557(7) \\ \mathrm{C}(6) & \mathrm{C}(7) & 1.523(6) & \mathrm{C}(6) & \mathrm{C}(1) & 1.380(8) \\ \mathrm{C}(6) & \mathrm{C}(5) & 1.379(8) & \mathrm{C}(12) & \mathrm{C}(13) & 1.557(7) \\ \mathrm{C}(13) & \mathrm{C}(14) & 1.508(8) & \mathrm{C}(1) & \mathrm{C}(2) & 1.411(7) \\ \mathrm{C}(2) & \mathrm{C}(3) & 1.37(1) & \mathrm{C}(17) & \mathrm{C}(16) & 1.526(8) \\ \mathrm{C}(14) & \mathrm{C}(15) & 1.505(8) & \mathrm{C}(3) & \mathrm{C}(4) & 1.37(1) \\ \mathrm{C}(15) & \mathrm{C}(16) & 1.540(7) & \mathrm{C}(4) & \mathrm{C}(5) & 1.394(9)\end{array}$


Table 5. Bond lengths involving hydrogens $(\AA)$

$\begin{array}{llllll}\text { atom } & \text { atom } & \text { distance } & \text { atom } & \text { atom } & \text { distance } \\ \mathrm{C}(11) & \mathrm{H}(8) & 0.950(7) & \mathrm{C}(12) & \mathrm{H}(9) & 0.950(7) \\ \mathrm{C}(12) & \mathrm{H}(10) & 0.950(8) & \mathrm{C}(13) & \mathrm{H}(11) & 0.950(8) \\ \mathrm{C}(13) & \mathrm{H}(12) & 0.950(8) & \mathrm{C}(7) & \mathrm{H}(6) & 0.950(8) \\ \mathrm{C}(7) & \mathrm{H}(7) & 0.950(7) & \mathrm{C}(1) & \mathrm{H}(1) & 0.950(8) \\ \mathrm{C}(2) & \mathrm{H}(2) & 0.950(9) & \mathrm{C}(17) & \mathrm{H}(18) & 0.950(8) \\ \mathrm{C}(17) & \mathrm{H}(19) & 0.950(8) & \mathrm{C}(17) & \mathrm{H}(20) & 0.95(1) \\ \mathrm{C}(14) & \mathrm{H}(13) & 0.950(9) & \mathrm{C}(14) & \mathrm{H}(14) & 0.950(8) \\ \mathrm{C}(3) & \mathrm{H}(3) & 0.950(9) & \mathrm{C}(15) & \mathrm{H}(15) & 0.950(8) \\ \mathrm{C}(15) & \mathrm{H}(16) & 0.950(8) & \mathrm{C}(16) & \mathrm{H}(17) & 0.950(8) \\ \mathrm{C}(4) & \mathrm{H}(4) & 0.95(1) & \mathrm{C}(5) & \mathrm{H}(5) & 0.950(9)\end{array}$


Table 6. Bond angles (0)

$\begin{array}{llllllll}\text { atom } & \text { atom } & \text { atom } & \text { angle } & \text { atom } & \text { atom } & \text { atom } & \text { angle } \\ \mathrm{N}(2) & \mathrm{C}(10) & \mathrm{C}(8) & 178.3(5) & \mathrm{C}(11) & \mathrm{C}(8) & \mathrm{C}(9) & 112.4(4) \\ \mathrm{C}(11) & \mathrm{C}(8) & \mathrm{C}(7) & 110.8(3) & \mathrm{C}(11) & \mathrm{C}(8) & \mathrm{C}(10) & 109.5(4) \\ \mathrm{C}(9) & \mathrm{C}(8) & \mathrm{C}(7) & 108.5(4) & \mathrm{C}(9) & \mathrm{C}(8) & \mathrm{C}(10) & 107.5(4) \\ \mathrm{C}(7) & \mathrm{C}(8) & \mathrm{C}(10) & 108.1(4) & \mathrm{C}(12) & \mathrm{C}(11) & \mathrm{C}(16) & 111.4(4) \\ \mathrm{C}(12) & \mathrm{C}(11) & \mathrm{C}(8) & 111.9(4) & \mathrm{C}(16) & \mathrm{C}(11) & \mathrm{C}(8) & 115.1(4) \\ \mathrm{C}(7) & \mathrm{C}(6) & \mathrm{C}(1) & 120.9(5) & \mathrm{C}(7) & \mathrm{C}(6) & \mathrm{C}(5) & 121.1(5) \\ \mathrm{C}(1) & \mathrm{C}(6) & \mathrm{C}(5) & 118.0(5) & \mathrm{N}(1) & \mathrm{C}(9) & \mathrm{C}(8) & 176.4(5) \\ \mathrm{C}(13) & \mathrm{C}(12) & \mathrm{C}(11) & 110.4(4) & \mathrm{C}(14) & \mathrm{C}(13) & \mathrm{C}(12) & 111.3(5) \\ \mathrm{C}(8) & \mathrm{C}(7) & \mathrm{C}(6) & 115.3(4) & \mathrm{C}(2) & \mathrm{C}(1) & \mathrm{C}(6) & 120.5(5) \\ \mathrm{C}(3) & \mathrm{C}(2) & \mathrm{C}(1) & 119.5(6) & \mathrm{C}(15) & \mathrm{C}(14) & \mathrm{C}(13) & 110.7(4) \\ \mathrm{C}(4) & \mathrm{C}(3) & \mathrm{C}(2) & 121.0(6) & \mathrm{C}(16) & \mathrm{C}(15) & \mathrm{C}(14) & 114.5(5) \\ \mathrm{C}(11) & \mathrm{C}(16) & \mathrm{C}(17) & 114.6(4) & \mathrm{C}(11) & \mathrm{C}(16) & \mathrm{C}(15) & 108.7(4) \\ \mathrm{C}(17) & \mathrm{C}(16) & \mathrm{C}(15) & 111.2(4) & \mathrm{C}(5) & \mathrm{C}(4) & \mathrm{C}(3) & 118.9(7) \\ \mathrm{C}(6) & \mathrm{C}(5) & \mathrm{C}(4) & 122.16) & & & & \end{array}$


Table 7. Bond angles involving hydrogens ( $\left.{ }^{\circ}\right)$

$\begin{array}{llllllll}\text { atom } & \text { atom } & \text { atom } & \text { angle } & \text { atom } & \text { atom } & \text { atom } & \text { angle } \\ \mathrm{C}(12) & \mathrm{C}(11) & \mathrm{H}(8) & 104.9(5) & \mathrm{C}(16) & \mathrm{C}(11) & \mathrm{H}(8) & 106.8(5) \\ \mathrm{H}(8) & \mathrm{C}(11) & \mathrm{C}(8) & 106.0(5) & \mathrm{C}(13) & \mathrm{C}(12) & \mathrm{H}(9) & 109.0(6) \\ \mathrm{C}(13) & \mathrm{C}(12) & \mathrm{H}(10) & 111.4(6) & \mathrm{H}(9) & \mathrm{C}(12) & \mathrm{H}(10) & 109.5(7) \\ \mathrm{H}(9) & \mathrm{C}(12) & \mathrm{C}(11) & 107.1(6) & \mathrm{H}(10) & \mathrm{C}(12) & \mathrm{C}(11) & 109.4(5) \\ \mathrm{C}(14) & \mathrm{C}(13) & \mathrm{H}(11) & 110.2(6) & \mathrm{C}(14) & \mathrm{C}(13) & \mathrm{H}(12) & 107.1(6) \\ \mathrm{H}(11) & \mathrm{C}(13) & \mathrm{H}(12) & 109.5(7) & \mathrm{H}(11) & \mathrm{C}(13) & \mathrm{C}(12) & 110.7(6) \\ \mathrm{H}(12) & \mathrm{C}(13) & \mathrm{C}(12) & 108.0(5) & \mathrm{H}(6) & \mathrm{C}(7) & \mathrm{H}(7) & 109.5(6) \\ \mathrm{H}(6) & \mathrm{C}(7) & \mathrm{C}(8) & 108.1(5) & \mathrm{H}(6) & \mathrm{C}(7) & \mathrm{C}(6) & 107.7(5) \\ \mathrm{H}(7) & \mathrm{C}(7) & \mathrm{C}(8) & 108.1(5) & \mathrm{H}(7) & \mathrm{C}(7) & \mathrm{C}(6) & 108.1(5) \\ \mathrm{C}(2) & \mathrm{C}(1) & \mathrm{H}(1) & 121.3(6) & \mathrm{H}(1) & \mathrm{C}(1) & \mathrm{C}(6) & 118.2(6) \\ \mathrm{C}(3) & \mathrm{C}(2) & \mathrm{H}(2) & 118.9(7) & \mathrm{H}(2) & \mathrm{C}(2) & \mathrm{C}(1) & 121.5(7) \\ \mathrm{C}(16) & \mathrm{C}(17) & \mathrm{H}(18) & 111.0(7) & \mathrm{C}(16) & \mathrm{C}(17) & \mathrm{H}(19) & 111.4(6) \\ \mathrm{C}(16) & \mathrm{C}(17) & \mathrm{H}(20) & 105.9(6) & \mathrm{H}(18) & \mathrm{C}(17) & \mathrm{H}(19) & 109.5(7) \\ \mathrm{H}(18) & \mathrm{C}(17) & \mathrm{H}(20) & 109.5(8) & \mathrm{H}(19) & \mathrm{C}(17) & \mathrm{H}(20) & 109.5(9) \\ \mathrm{C}(15) & \mathrm{C}(14) & \mathrm{H}(13) & 105.4(6) & \mathrm{C}(15) & \mathrm{C}(14) & \mathrm{H}(14) & 113.3(7) \\ \mathrm{H}(13) & \mathrm{C}(14) & \mathrm{H}(14) & 109.5(7) & \mathrm{H}(13) & \mathrm{C}(14) & \mathrm{C}(13) & 106.5(6) \\ \mathrm{H}(14) & \mathrm{C}(14) & \mathrm{C}(13) & 111.1(6) & \mathrm{C}(4) & \mathrm{C}(3) & \mathrm{H}(3) & 120(1) \\ \mathrm{H}(3) & \mathrm{C}(3) & \mathrm{C}(2) & 118.6(9) & \mathrm{C}(16) & \mathrm{C}(15) & \mathrm{H}(15) & 109.8(5) \\ \mathrm{C}(16) & \mathrm{C}(15) & \mathrm{H}(16) & 109.0(5) & \mathrm{H}(15) & \mathrm{C}(15) & \mathrm{H}(16) & 109.5(7) \\ \mathrm{H}(15) & \mathrm{C}(15) & \mathrm{C}(14) & 108.5(6) & \mathrm{H}(16) & \mathrm{C}(15) & \mathrm{C}(14) & 105.5(6) \\ \mathrm{H}(17) & \mathrm{C}(16) & \mathrm{C}(11) & 107.9(5) & \mathrm{H}(17) & \mathrm{C}(16) & \mathrm{C}(17) & 105.6(5) \\ \mathrm{H}(17) & \mathrm{C}(16) & \mathrm{C}(15) & 108.6(6) & \mathrm{C}(5) & \mathrm{C}(4) & \mathrm{H}(4) & 123.2(8) \\ \mathrm{H}(4) & \mathrm{C}(4) & \mathrm{C}(3) & 117.9(8) & \mathrm{H}(5) & \mathrm{C}(5) & \mathrm{C}(6) & 118.6(7) \\ \mathrm{H}(5) & \mathrm{C}(5) & \mathrm{C}(4) & 119.3(7) & & & & \end{array}$


Table 8. Torsion Angles( $\left.{ }^{0}\right)$

atom1 atom2 atom3 atom4 angle atom1 atom2 atom3 atom4 angle

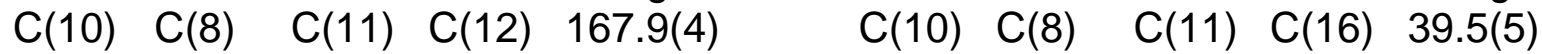

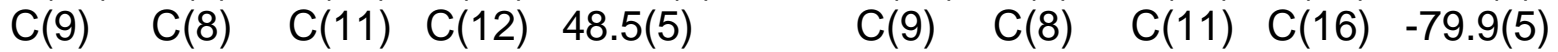

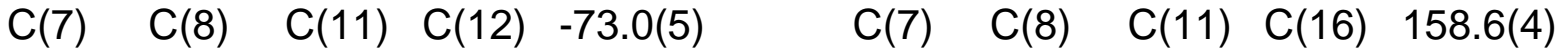

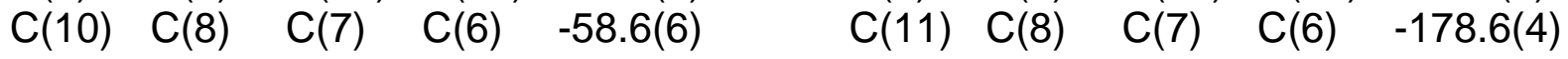

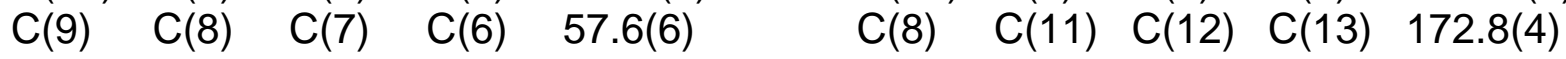

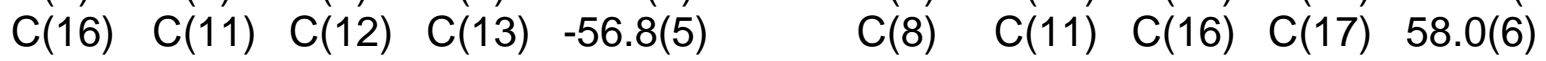

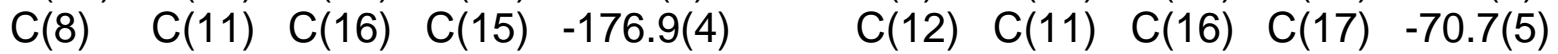

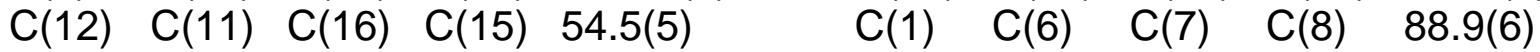

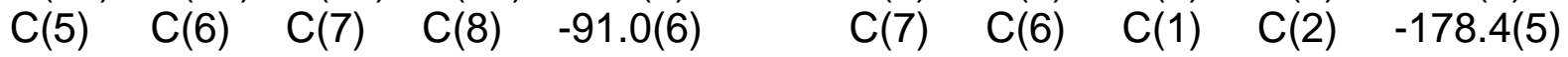

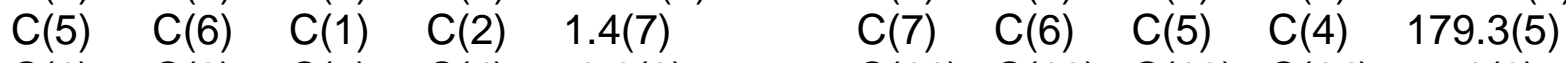

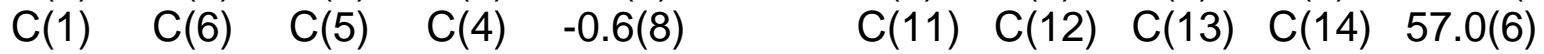

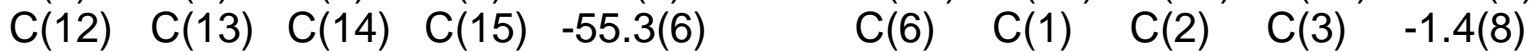

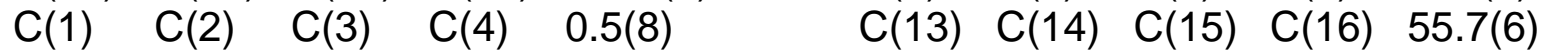

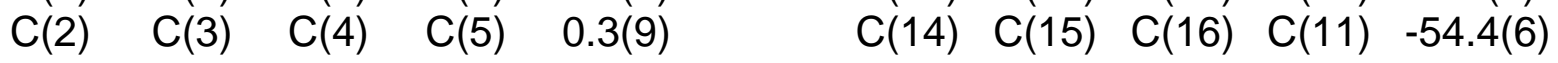

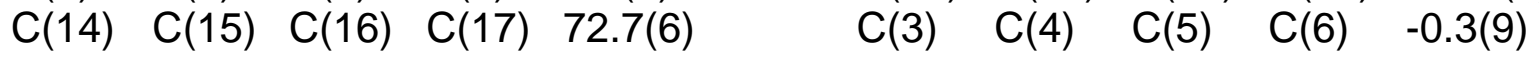

The sign is positive if when looking from atom 2 to atom 3 a clock-wise motion of atom 1 would superimpose it on atom 4. 
Table 9. Torsion Angles involving hydrogens( $\left({ }^{0}\right)$

\begin{tabular}{|c|c|c|c|c|c|c|c|c|c|}
\hline & & & & & & & & & \\
\hline & (8) & $C(11)$ & $H(8)$ & $-78.4(5)$ & $C(9)$ & $C(8)$ & $C(11)$ & $\mathrm{H}(8)$ & 162.3(5) \\
\hline (7) & $C(8)$ & $C(11)$ & $H(8)$ & $40.8(6)$ & $C(10)$ & $C(8)$ & $\mathrm{C}(7)$ & $H(6)$ & $61.9(6)$ \\
\hline$C(10)$ & $C(8)$ & $C(7)$ & $\mathrm{H}(7)$ & $-179.7(5)$ & $C(11)$ & $C(8)$ & $\mathrm{C}(7)$ & $H(6)$ & $-58.1(6)$ \\
\hline$C(11)$ & $C(8)$ & $C(7)$ & $\mathrm{H}(7)$ & $60.3(6)$ & C(9) & $C(8)$ & $\mathrm{C}(7)$ & $H(6)$ & $178.2(5)$ \\
\hline$C(9)$ & $C(8)$ & $C(7)$ & $\mathrm{H}(7)$ & $-63.4(6)$ & $C(8)$ & $C(11)$ & $C(12)$ & $H(9)$ & $-68.6(6)$ \\
\hline$C(8)$ & $C(11)$ & $C(12)$ & $H(10)$ & 49.9(7) & $C(16)$ & $C(11)$ & $C(12)$ & $H(9)$ & $61.7(6)$ \\
\hline$C(16)$ & $C(11)$ & $C(12)$ & $H(10)$ & $-179.8(5)$ & $H(8)$ & $C(11)$ & $C(12)$ & $C(13)$ & $58.3(6)$ \\
\hline$H(8)$ & $C(11)$ & $C(12)$ & $\mathrm{H}(9)$ & $176.9(6)$ & $H(8)$ & $C(11)$ & $C(12)$ & $H(10)$ & $-64.6(7)$ \\
\hline$C(8)$ & $C(11)$ & $C(16)$ & $\mathrm{H}(17)$ & $-59.3(6)$ & $C(12)$ & $C(11)$ & $C(16)$ & $H(17)$ & 172.1(5) \\
\hline$H(8)$ & $C(11)$ & $C(16)$ & $C(17)$ & 175.3(5) & $\mathrm{H}(8)$ & $C(11)$ & $C(16)$ & $C(15)$ & $-59.5(6)$ \\
\hline $\mathrm{H}(8)$ & $C(11)$ & $C(16)$ & $H(17)$ & $58.1(7)$ & $C(1)$ & $C(6)$ & $C(7)$ & $H(6)$ & $-31.9(7)$ \\
\hline$C(1)$ & $C(6)$ & $\mathrm{C}(7)$ & $\mathrm{H}(7)$ & $-150.1(6)$ & $C(5)$ & $C(6)$ & $\mathrm{C}(7)$ & $H(6)$ & $148.2(5)$ \\
\hline$C(5)$ & $C(6)$ & $C(7)$ & $\mathrm{H}(7)$ & $30.0(7)$ & $C(7)$ & $C(6)$ & $C(1)$ & $H(1)$ & $2.0(8)$ \\
\hline$C(5)$ & $C(6)$ & $C(1)$ & $H(1)$ & $-178.1(6)$ & $\mathrm{C}(7)$ & $C(6)$ & $C(5)$ & $\mathrm{H}(5)$ & $1.3(8)$ \\
\hline$C(1)$ & $C(6)$ & $C(5)$ & $H(5)$ & $-178.6(6)$ & $C(11)$ & $C(12)$ & $C(13)$ & $H(11)$ & $179(804)$ \\
\hline$C(11)$ & $C(12)$ & $C(13)$ & $H(12)$ & $-60.3(7)$ & $H(9)$ & $C(12)$ & $C(13)$ & $C(14)$ & $-60.4(7)$ \\
\hline $\mathrm{H}(9)$ & $C(12)$ & $C(13)$ & $H(11)$ & $62.6(8)$ & $H(9)$ & $C(12)$ & $C(13)$ & $H(12)$ & $-177.6(7)$ \\
\hline$H(10)$ & $C(12)$ & $C(13)$ & $C(14)$ & $178.8(6)$ & $H(10)$ & $C(12)$ & $C(13)$ & $H(11)$ & $-58.3(8)$ \\
\hline$H(10)$ & $C(12)$ & $C(13)$ & $H(12)$ & $61.5(8)$ & $C(12)$ & $C(13)$ & $C(14)$ & $H(13)$ & $58.8(7)$ \\
\hline$C(12)$ & $C(13)$ & $C(14)$ & $H(14)$ & $177.9(7)$ & $H(11)$ & C(13) & $C(14)$ & $C(15)$ & $-178.5(6)$ \\
\hline$H(11)$ & $C(13)$ & $C(14)$ & $H(13)$ & $-64.4(8)$ & $H(11)$ & C(13) & $C(14)$ & $H(14)$ & $54.7(9)$ \\
\hline $\mathrm{H}(12)$ & $C(13)$ & $C(14)$ & $C(15)$ & 62.5(7) & $\mathrm{H}(12)$ & $C(13)$ & $C(14)$ & $\mathrm{H}(13)$ & $176.6(7)$ \\
\hline$H(12)$ & $C(13)$ & $C(14)$ & $\mathrm{H}(14)$ & $-64.3(9)$ & $\mathrm{C}(6)$ & $\mathrm{C}(1)$ & $C(2)$ & $\mathrm{H}(2)$ & 179(359) \\
\hline $\mathrm{H}(1)$ & C(1) & $\mathrm{C}(2)$ & C(3) & $178.1(6)$ & $H(1)$ & $C(1)$ & $C(2)$ & $H(2)$ & $0(1)$ \\
\hline$C(1)$ & $C(2)$ & $C(3)$ & $\mathrm{H}(3)$ & 179(359) & $H(2)$ & $C(2)$ & $C(3)$ & $C(4)$ & 179.4(7) \\
\hline $\mathrm{H}(2)$ & $C(2)$ & $C(3)$ & $H(3)$ & $-1(1)$ & $H(18)$ & $C(17)$ & $C(16)$ & $C(11)$ & $-179.6(6)$ \\
\hline$H(18)$ & $C(17)$ & $C(16)$ & $C(15)$ & $56.6(8)$ & $H(18)$ & $C(17)$ & $C(16)$ & $H(17)$ & $-61.1(8)$ \\
\hline $\mathrm{H}(19)$ & $C(17)$ & $C(16)$ & $C(11)$ & $-57.4(8)$ & $H(19)$ & $C(17)$ & $C(16)$ & $C(15)$ & 178.8(7) \\
\hline$H(19)$ & $C(17)$ & $C(16)$ & $H(17)$ & 61.2(9) & $H(20)$ & C(17) & $C(16)$ & $C(11)$ & $61.6(7)$ \\
\hline$H(20)$ & $C(17)$ & $C(16)$ & $C(15)$ & $-62.2(7)$ & $H(20)$ & $C(17)$ & $C(16)$ & $H(17)$ & $-179(719)$ \\
\hline$C(13)$ & $C(14)$ & $C(15)$ & $H(15)$ & $178.7(6)$ & $C(13)$ & $C(14)$ & $C(15)$ & $H(16)$ & $-64.1(7)$ \\
\hline $\mathrm{H}(13)$ & $C(14)$ & $C(15)$ & $C(16)$ & $-59.1(7)$ & $H(13)$ & $C(14)$ & $C(15)$ & $H(15)$ & $63.9(8)$ \\
\hline$H(13)$ & $C(14)$ & $C(15)$ & $H(16)$ & $-178.9(7)$ & $H(14)$ & $C(14)$ & $C(15)$ & $C(16)$ & $-178.8(6)$ \\
\hline$H(14)$ & $C(14)$ & $C(15)$ & $H(15)$ & $-55.8(9)$ & $H(14)$ & $C(14)$ & $C(15)$ & $H(16)$ & $61.4(8)$ \\
\hline$C(2)$ & C(3) & C(4) & $\mathrm{H}(4)$ & $-179(508)$ & $H(3)$ & $C(3)$ & $\mathrm{C}(4)$ & C(5) & $-178.9(7)$ \\
\hline $\mathrm{H}(3)$ & $C(3)$ & $C(4)$ & $\mathrm{H}(4)$ & $1(1)$ & $C(14)$ & $C(15)$ & $C(16)$ & $H(17)$ & $-171.6(6)$ \\
\hline$H(15)$ & $C(15)$ & $C(16)$ & $C(11)$ & $-176.7(6)$ & $H(15)$ & $C(15)$ & $C(16)$ & $C(17)$ & $-49.6(8)$ \\
\hline
\end{tabular}


Table 9. Torsion angles involving hydrogens $(0)$-- continued

atom1 atom2 atom3 atom4 angle atom1 atom2 atom3 atom4 angle

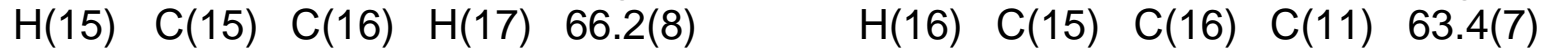

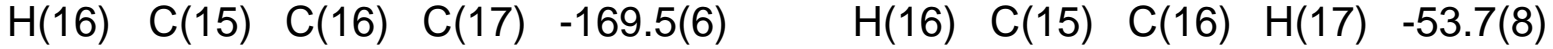

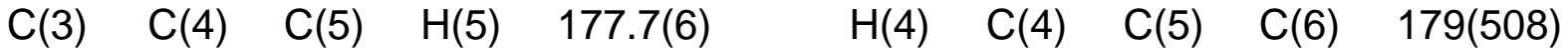

$\mathrm{H}(4) \quad \mathrm{C}(4) \quad \mathrm{C}(5) \quad \mathrm{H}(5) \quad-2(1)$

The sign is positive if when looking from atom 2 to atom 3 a clock-wise motion of atom 1 would superimpose it on atom 4. 


\section{X-ray crystallographic Data of endo-6d}

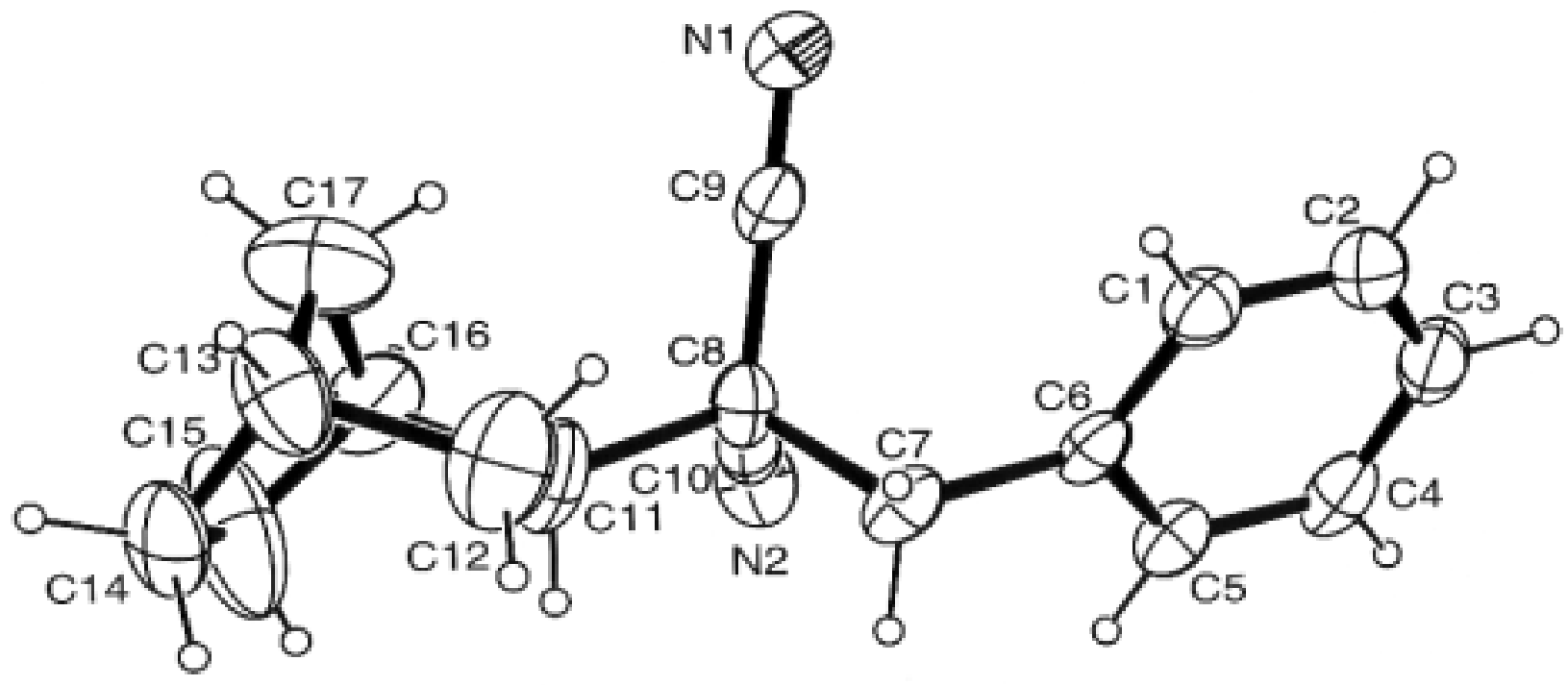

Figure S5. ORTEP drawing of endo-6d (ellipsoids at 20\% probability). 


\section{Experimental}

\section{$\underline{\text { Data Collection }}$}

A colorless platelet crystal of $\mathrm{C}_{17} \mathrm{H}_{18} \mathrm{~N}_{2}$ having approximate dimensions of 0.60 $\times 0.50 \times 0.05 \mathrm{~mm}$ was mounted on a glass fiber. All measurements were made on a Rigaku RAXIS RAPID imaging plate area detector with graphite monochromated Mo-Ka radiation.

Indexing was performed from 30 oscillations that were exposed for 36 seconds. The crystal-to-detector distance was $127.40 \mathrm{~mm}$.

Cell constants and an orientation matrix for data collection corresponded to a primitive orthorhombic cell with dimensions:

$$
\begin{aligned}
& a=17.61(2) \AA \\
& \mathrm{b}=\text { 9.93(1) } \AA \\
& \mathrm{c}=8.376(9) \AA \\
& V=1463(2) \AA^{3}
\end{aligned}
$$

For $Z=4$ and F.W. $=250.34$, the calculated density is $1.14 \mathrm{~g} / \mathrm{cm}^{3}$. Based on the systematic absences of:

$$
\begin{array}{ll}
\text { Okl: } & \mathrm{k}+\mathrm{l} \pm 2 \mathrm{n} \\
\mathrm{hOl}: & \mathrm{h} \pm 2 \mathrm{n}
\end{array}
$$

packing considerations, a statistical analysis of intensity distribution, and the successful solution and refinement of the structure, the space group was determined to be:

$$
\text { Pna21 (\#33) }
$$

The data were collected at a temperature of $23 \pm 1^{\circ} \mathrm{C}$ to a maximum $2 \theta$ value of 54.90. A total of 44 oscillation images were collected. A sweep of data was done using $\omega$ scans from 130.0 to $190.0^{\circ}$ in $5.0^{\circ}$ step, at $\chi=45.0^{\circ}$ and $\phi=0.0^{\circ}$. The exposure rate was 150.0 [sec./0]. A second sweep was performed using $\omega$ scans from 0.0 to $160.0^{\circ}$ in 
5.00 step, at $\chi=45.00$ and $\phi=180.00$. The exposure rate was 150.0 [sec. $/ 0$ ]. The crystal-to-detector distance was $127.40 \mathrm{~mm}$. Readout was performed in the $0.100 \mathrm{~mm}$ pixel mode.

\section{$\underline{\text { Data Reduction }}$}

Of the 28490 reflections that were collected, 1946 were unique $\left(R_{\text {int }}=0.056\right)$; equivalent reflections were merged.

The linear absorption coefficient, $\mu$, for Mo-K $\alpha$ radiation is $0.7 \mathrm{~cm}^{-1}$. The data were corrected for Lorentz and polarization effects.

\section{$\underline{\text { Structure Solution and Refinement }}$}

The structure was solved by direct methods ${ }^{1}$ and expanded using Fourier techniques $^{2}$. The non-hydrogen atoms were refined anisotropically. Hydrogen atoms were refined using the riding model. The final cycle of full-matrix least-squares refinement ${ }^{3}$ on $\mathrm{F}^{2}$ was based on 1788 observed reflections and 190 variable parameters and converged (largest parameter shift was 0.00 times its esd) with unweighted and weighted agreement factors of:

$$
\begin{gathered}
\mathrm{R} 1=\Sigma\|\mathrm{Fo}|-| \mathrm{Fc}\| / \Sigma|\mathrm{Fo}|=0.098 \\
\mathrm{wR} 2=\left[\Sigma\left(\mathrm{w}\left(\mathrm{Fo}^{2}-\mathrm{Fc}^{2}\right)^{2}\right) / \Sigma \mathrm{w}\left(\mathrm{Fo}^{2}\right)^{2}\right]^{1 / 2}=0.223
\end{gathered}
$$

The standard deviation of an observation of unit weight 4 was 1.23. A Sheldrick weighting scheme was used. Plots of $\Sigma \mathrm{w}(|\mathrm{Fo}|-|\mathrm{Fc}|)^{2}$ versus $|\mathrm{Fo}|$, reflection order in data collection, $\sin \theta / \lambda$ and various classes of indices showed no unusual trends. The maximum and minimum peaks on the final difference Fourier map corresponded to 0.43 and $-0.46 \mathrm{e}^{-} / \AA^{3}$, respectively.

Neutral atom scattering factors were taken from Cromer and Waber 5 . Anomalous dispersion effects were included in Fcalc 6 ; the values for $\Delta f^{\prime}$ and $\Delta f^{\prime \prime}$ were those of Creagh and McAuley 7 . The values for the mass attenuation coefficients are those of Creagh and Hubbell 8 . All calculations were performed using the CrystalStructure 9,10 crystallographic software package. 


\section{References}

(1) SHELX97: Sheldrick, G.M. (1997).

(2) DIRDIF99: Beurskens, P.T., Admiraal, G., Beurskens, G., Bosman, W.P., de Gelder, R., Israel, R. and Smits, J.M.M.(1999). The DIRDIF-99 program system, Technical Report of the Crystallography Laboratory, University of Nijmegen, The Netherlands.

(3) Least Squares function minimized:

$$
\Sigma w\left(\mathrm{~F}_{\mathrm{o}}{ }^{2}-\mathrm{F}_{\mathrm{c}}{ }^{2}\right)^{2} \quad \text { where } \mathrm{w}=\text { Least Squares weights. }
$$

(4) Standard deviation of an observation of unit weight:

$$
\begin{aligned}
& {\left[\sum w\left(\mathrm{~F}_{0}{ }^{2}-\mathrm{F}_{\mathrm{C}}{ }^{2}\right)^{2} /\left(\mathrm{N}_{\mathrm{O}}-\mathrm{N}_{\mathrm{V}}\right)\right]^{1 / 2}} \\
& \text { where: } \quad \mathrm{N}_{\mathrm{O}}=\text { number of observations } \\
& \mathrm{N}_{\mathrm{V}}=\text { number of variables }
\end{aligned}
$$

(5) Cromer, D. T. \& Waber, J. T.; "International Tables for X-ray Crystallography", Vol. IV, The Kynoch Press, Birmingham, England, Table 2.2 A (1974).

(6) Ibers, J. A. \& Hamilton, W. C.; Acta Crystallogr., 17, 781 (1964).

(7) Creagh, D. C. \& McAuley, W.J .; "International Tables for Crystallography", Vol C, (A.J.C. Wilson, ed.), Kluwer Academic Publishers, Boston, Table 4.2.6.8, pages 219-222 (1992).

(8) Creagh, D. C. \& Hubbell, J.H..; "International Tables for Crystallography", Vol C, (A.J.C. Wilson, ed.), Kluwer Academic Publishers, Boston, Table 4.2.4.3, pages 200-206 (1992).

(9) CrystalStructure 3.5.1: Crystal Structure Analysis Package, Rigaku and Rigaku/MSC (2000-2003). 9009 New Trails Dr. The Woodlands TX 77381 USA.

(10) CRYSTALS Issue 10: Watkin, D.J., Prout, C.K. Carruthers, J.R. \& Betteridge, P.W. Chemical Crystallography Laboratory, Oxford, UK. (1996) 
EXPERIMENTAL DETAILS

\author{
A. Crystal Data
}

Empirical Formula

Formula Weight

Crystal Color, Habit

Crystal Dimensions

Crystal System

Lattice Type

Indexing Images

Detector Position

Pixel Size

Lattice Parameters

Space Group

$Z$ value

$D_{\text {calc }}$

$F_{000}$

$\mu(\operatorname{MoK} \alpha)$
$\mathrm{C}_{17} \mathrm{H}_{18} \mathrm{~N}_{2}$

250.34

colorless, platelet

$0.60 \times 0.50 \times 0.05 \mathrm{~mm}$

orthorhombic

Primitive

3 oscillations @ 36.0 seconds

$127.40 \mathrm{~mm}$

$0.100 \mathrm{~mm}$

$$
\begin{aligned}
& \mathrm{a}=17.61(2) \AA \\
& \mathrm{b}=9.93(1) \AA \\
& \mathrm{c}=8.376(9) \AA \\
& \mathrm{V}=1463(2) \AA^{3}
\end{aligned}
$$

Pna21 (\#33)

4

$1.136 \mathrm{~g} / \mathrm{cm}^{3}$

536.00

$0.67 \mathrm{~cm}^{-1}$ 
B. Intensity Measurements

Diffractometer

Radiation

Detector Aperture

Data Images

$\omega$ oscillation Range $(\chi=45.0, \phi=0.0)$

Exposure Rate

$\omega$ oscillation Range ( $\chi=45.0, \phi=180.0)$

Exposure Rate

Detector Position

Pixel Size

$2 \theta_{\max }$

No. of Reflections Measured

Corrections
Rigaku RAXIS-RAPID

$\operatorname{MoK} \alpha(\lambda=0.71075 \AA)$

graphite monochromated

$270 \mathrm{~mm} \times 256 \mathrm{~mm}$

44 exposures

$130.0-190.00$

$150.0 \mathrm{sec} . / 0$

$0.0-160.00$

$150.0 \mathrm{sec} . / \mathrm{O}$

$127.40 \mathrm{~mm}$

$0.100 \mathrm{~mm}$

54.90

Total: 28490

Unique: $1946\left(R_{\text {int }}=0.056\right)$ Lorentz-polarization 
C. Structure Solution and Refinement

Structure Solution

Refinement

Function Minimized

Least Squares Weights

Fo2)

Anomalous Dispersion

No. Observations (All reflections)

No. Variables

Reflection/Parameter Ratio

Residuals: R (All reflections)

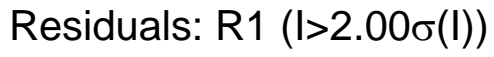

Residuals: wR2 (All reflections)

Goodness of Fit Indicator

Max Shift/Error in Final Cycle

Maximum peak in Final Diff. Map

Minimum peak in Final Diff. Map
Direct Methods (SHELX97)

Full-matrix least-squares on $\mathrm{F}^{2}$

$\Sigma \mathrm{w}\left(\mathrm{Fo}^{2}-\mathrm{Fc}^{2}\right)^{2}$

$1 /\left[0.0020 \mathrm{Fo}^{2}+3.0000 \sigma\left(\mathrm{Fo}^{2}\right)+0.5000\right] /(4$

All non-hydrogen atoms

1788

190

9.41

0.142

0.098

0.223

1.228

0.000

$0.43 \mathrm{e}^{-/ \AA^{3}}$

$-0.46 e^{-} / \AA^{3}$ 
Table 1. Atomic coordinates and $\mathrm{B}_{\mathrm{iso}} / \mathrm{B}_{\text {eq }}$

$\begin{array}{lllll}\text { atom } & \mathrm{x} & \mathrm{y} & \mathrm{z} & \mathrm{B}_{\mathrm{eq}} \\ \mathrm{N}(1) & 0.1745(3) & -0.0585(6) & 0.6965(9) & 8.5(2) \\ \mathrm{N}(2) & 0.3244(3) & -0.0924(7) & 0.3006(9) & 8.6(2) \\ \mathrm{C}(1) & 0.0940(4) & 0.1569(6) & 0.4046(8) & 6.9(2) \\ \mathrm{C}(2) & 0.0328(4) & 0.1291(7) & 0.311(1) & 7.7(2) \\ \mathrm{C}(3) & 0.0407(5) & 0.1040(6) & 0.154(1) & 8.0(2) \\ \mathrm{C}(4) & 0.1116(5) & 0.1045(6) & 0.0854(9) & 7.7(2) \\ \mathrm{C}(5) & 0.1736(4) & 0.1341(6) & 0.1792(8) & 6.8(2) \\ \mathrm{C}(6) & 0.1680(3) & 0.1603(5) & 0.3421(7) & 5.6(1) \\ \mathrm{C}(7) & 0.2349(3) & 0.1954(5) & 0.4430(7) & 6.5(1) \\ \mathrm{C}(8) & 0.2747(3) & 0.0719(6) & 0.5232(8) & 6.2(1) \\ \mathrm{C}(9) & 0.2179(4) & -0.0032(6) & 0.6204(8) & 6.4(1) \\ \mathrm{C}(10) & 0.3032(3) & -0.0232(6) & 0.3980(9) & 6.5(2) \\ \mathrm{C}(11) & 0.3482(5) & 0.1205(7) & 0.619(1) & 9.9(2) \\ \mathrm{C}(12) & 0.3382(8) & 0.220(1) & 0.743(1) & 15.0(4) \\ \mathrm{C}(13) & 0.3878(5) & 0.170(1) & 0.885(1) & 10.9(3) \\ \mathrm{C}(14) & 0.4646(4) & 0.1831(8) & 0.836(1) & 9.8(2) \\ \mathrm{C}(15) & 0.4706(5) & 0.073(2) & 0.708(2) & 16.6(5) \\ \mathrm{C}(16) & 0.4014(7) & 0.0080(7) & 0.697(1) & 11.0(3) \\ \mathrm{C}(17) & 0.3668(7) & 0.030(1) & 0.860(2) & 13.6(4)\end{array}$

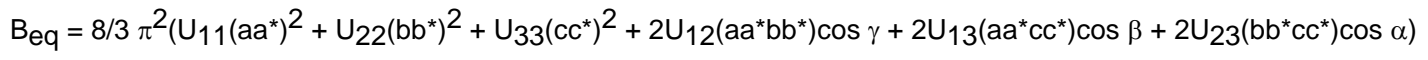


Table 2. Atomic coordinates and $B$ iso involving hydrogens $/ B_{\text {eq }}$

$\begin{array}{lclll}\text { atom } & x & y & z & B_{e q} \\ H(1) & 0.0871(4) & 0.1810(6) & 0.5134(8) & 8.3(2) \\ H(2) & -0.0166(4) & 0.1250(7) & 0.356(1) & 9.3(2) \\ \mathrm{H}(3) & -0.0027(5) & 0.0841(6) & 0.091(1) & 9.6(2) \\ \mathrm{H}(4) & 0.1158(5) & 0.0872(6) & -0.0258(9) & 9.2(2) \\ \mathrm{H}(5) & 0.2230(4) & 0.1366(6) & 0.1333(8) & 8.1(2) \\ \mathrm{H}(6) & 0.2712(3) & 0.2354(5) & 0.3739(7) & 7.8(2) \\ \mathrm{H}(7) & 0.2204(3) & 0.2586(5) & 0.5226(7) & 7.8(2) \\ \mathrm{H}(8) & 0.3794(5) & 0.1626(7) & 0.541(1) & 11.9(3) \\ \mathrm{H}(9) & 0.3519(8) & 0.309(1) & 0.715(1) & 18.1(5) \\ \mathrm{H}(10) & 0.2851(8) & 0.216(1) & 0.764(1) & 18.0(5) \\ \mathrm{H}(11) & 0.3766(5) & 0.209(1) & 0.986(1) & 13.1(3) \\ \mathrm{H}(12) & 0.4741(4) & 0.2698(8) & 0.792(1) & 11.8(3) \\ \mathrm{H}(13) & 0.5002(4) & 0.1653(8) & 0.918(1) & 11.8(3) \\ \mathrm{H}(14) & 0.4803(5) & 0.114(2) & 0.608(2) & 19.9(6) \\ \mathrm{H}(15) & 0.5102(5) & 0.012(2) & 0.733(2) & 19.9(6) \\ \mathrm{H}(16) & 0.3981(7) & -0.0802(7) & 0.653(1) & 13.2(3) \\ \mathrm{H}(17) & 0.3833(7) & -0.031(1) & 0.940(2) & 16.3(4) \\ \mathrm{H}(18) & 0.3132(7) & 0.025(1) & 0.849(2) & 16.3(4)\end{array}$

$B_{e q}=8 / 3 \pi^{2}\left(U_{11}\left(a a^{\star}\right)^{2}+U_{22}\left(b b^{\star}\right)^{2}+U_{33}\left(c c^{\star}\right)^{2}+2 U_{12}\left(a a^{*} b b^{\star}\right) \cos \gamma+2 U_{13}\left(a a^{*} c^{*}\right) \cos \beta+2 U_{23}\left(b b^{*} c c^{\star}\right) \cos \alpha\right)$ 
Table 3. Anisotropic Displacement Parameters

$\begin{array}{lllllrr}\text { atom } & \mathrm{U}_{11} & \mathrm{U}_{22} & \mathrm{U}_{33} & \mathrm{U}_{12} & \mathrm{U}_{13} & \mathrm{U}_{23} \\ \mathrm{~N}(1) & 0.110(4) & 0.109(4) & 0.102(4) & -0.001(3) & -0.009(4) & 0.035(4) \\ \mathrm{N}(2) & 0.101(4) & 0.111(4) & 0.114(5) & 0.005(3) & -0.013(4) & -0.023(4) \\ \mathrm{C}(1) & 0.101(4) & 0.085(4) & 0.078(4) & 0.017(3) & 0.006(4) & 0.006(3) \\ \mathrm{C}(2) & 0.087(4) & 0.106(5) & 0.101(6) & 0.006(3) & 0.000(4) & 0.018(4) \\ \mathrm{C}(3) & 0.112(6) & 0.084(4) & 0.108(6) & 0.005(4) & -0.041(5) & 0.017(4) \\ \mathrm{C}(4) & 0.129(6) & 0.091(4) & 0.071(4) & 0.018(4) & -0.020(5) & 0.016(3) \\ \mathrm{C}(5) & 0.099(4) & 0.077(3) & 0.080(4) & 0.012(3) & -0.006(4) & 0.014(3) \\ \mathrm{C}(6) & 0.092(4) & 0.055(3) & 0.065(3) & 0.005(3) & -0.010(3) & 0.010(3) \\ \mathrm{C}(7) & 0.108(4) & 0.060(3) & 0.078(4) & 0.007(3) & -0.005(4) & 0.010(3) \\ \mathrm{C}(8) & 0.080(3) & 0.083(4) & 0.071(4) & 0.001(3) & -0.021(3) & -0.009(3) \\ \mathrm{C}(9) & 0.098(4) & 0.071(3) & 0.073(3) & 0.007(3) & -0.020(4) & 0.014(3) \\ \mathrm{C}(10) & 0.071(3) & 0.079(4) & 0.096(5) & 0.002(3) & -0.018(3) & 0.004(4) \\ \mathrm{C}(11) & 0.169(7) & 0.099(5) & 0.109(6) & 0.002(5) & -0.073(6) & -0.002(5) \\ \mathrm{C}(12) & 0.27(1) & 0.162(9) & 0.142(8) & 0.075(9) & -0.044(9) & -0.040(8) \\ \mathrm{C}(13) & 0.121(6) & 0.170(9) & 0.124(7) & 0.006(6) & -0.024(6) & -0.072(6) \\ \mathrm{C}(14) & 0.118(6) & 0.108(5) & 0.148(7) & -0.007(4) & -0.057(6) & -0.036(5) \\ \mathrm{C}(15) & 0.116(6) & 0.29(1) & 0.22(1) & -0.006(8) & -0.028(8) & -0.14(1) \\ \mathrm{C}(16) & 0.23(1) & 0.065(4) & 0.117(6) & -0.026(5) & -0.039(7) & -0.007(4) \\ \mathrm{C}(17) & 0.21(1) & 0.124(7) & 0.18(1) & -0.009(7) & 0.052(9) & -0.014(8)\end{array}$

The general temperature factor expression: $\exp \left(-2 \pi^{2}\left(a^{*} U_{11} h^{2}+b^{*} U_{22} k^{2}+\left.c^{*} U_{33}\right|^{2}\right.\right.$ $\left.\left.+2 a^{*} b^{*} U_{12} h k+2 a^{*} c^{*} U_{13} h l+2 b^{*} c^{*} U_{23} k l\right)\right)$ 
Table 4. Bond lengths $(\AA)$

$\begin{array}{llllll}\text { atom } & \text { atom } & \text { distance } & \text { atom } & \text { atom } & \text { distance } \\ \mathrm{N}(1) & \mathrm{C}(9) & 1.135(9) & \mathrm{N}(2) & \mathrm{C}(10) & 1.13(1) \\ \mathrm{C}(1) & \mathrm{C}(2) & 1.36(1) & \mathrm{C}(1) & \mathrm{C}(6) & 1.404(9) \\ \mathrm{C}(2) & \mathrm{C}(3) & 1.34(1) & \mathrm{C}(3) & \mathrm{C}(4) & 1.38(1) \\ \mathrm{C}(4) & \mathrm{C}(5) & 1.38(1) & \mathrm{C}(5) & \mathrm{C}(6) & 1.393(9) \\ \mathrm{C}(6) & \mathrm{C}(7) & 1.491(8) & \mathrm{C}(7) & \mathrm{C}(8) & 1.564(8) \\ \mathrm{C}(8) & \mathrm{C}(9) & 1.490(9) & \mathrm{C}(8) & \mathrm{C}(10) & 1.498(9) \\ \mathrm{C}(8) & \mathrm{C}(11) & 1.60(1) & \mathrm{C}(11) & \mathrm{C}(12) & 1.44(1) \\ \mathrm{C}(11) & \mathrm{C}(16) & 1.60(1) & \mathrm{C}(12) & \mathrm{C}(13) & 1.55(2) \\ \mathrm{C}(13) & \mathrm{C}(14) & 1.42(1) & \mathrm{C}(13) & \mathrm{C}(17) & 1.45(1) \\ \mathrm{C}(14) & \mathrm{C}(15) & 1.53(2) & \mathrm{C}(15) & \mathrm{C}(16) & 1.38(2) \\ \mathrm{C}(16) & \mathrm{C}(17) & 1.51(2) & & & \end{array}$


Table 5. Bond lengths involving hydrogens $(\AA)$

$\begin{array}{llllll}\text { atom } & \text { atom } & \text { distance } & \text { atom } & \text { atom } & \text { distance } \\ \mathrm{C}(1) & \mathrm{H}(1) & 0.95(1) & \mathrm{C}(2) & \mathrm{H}(2) & 0.95(1) \\ \mathrm{C}(3) & \mathrm{H}(3) & 0.95(1) & \mathrm{C}(4) & \mathrm{H}(4) & 0.95(1) \\ \mathrm{C}(5) & \mathrm{H}(5) & 0.950(9) & \mathrm{C}(7) & \mathrm{H}(6) & 0.950(8) \\ \mathrm{C}(7) & \mathrm{H}(7) & 0.950(8) & \mathrm{C}(11) & \mathrm{H}(8) & 0.95(1) \\ \mathrm{C}(12) & \mathrm{H}(9) & 0.95(2) & \mathrm{C}(12) & \mathrm{H}(10) & 0.95(2) \\ \mathrm{C}(13) & \mathrm{H}(11) & 0.95(1) & \mathrm{C}(14) & \mathrm{H}(12) & 0.95(1) \\ \mathrm{C}(14) & \mathrm{H}(13) & 0.95(1) & \mathrm{C}(15) & \mathrm{H}(14) & 0.95(2) \\ \mathrm{C}(15) & \mathrm{H}(15) & 0.95(2) & \mathrm{C}(16) & \mathrm{H}(16) & 0.95(1) \\ \mathrm{C}(17) & \mathrm{H}(17) & 0.95(2) & \mathrm{C}(17) & \mathrm{H}(18) & 0.95(2)\end{array}$


Table 6. Bond angles (0)

$\begin{array}{llllllll}\text { atom } & \text { atom } & \text { atom } & \text { angle } & \text { atom } & \text { atom } & \text { atom } & \text { angle } \\ \mathrm{C}(2) & \mathrm{C}(1) & \mathrm{C}(6) & 121.5(6) & \mathrm{C}(3) & \mathrm{C}(2) & \mathrm{C}(1) & 121.3(7) \\ \mathrm{C}(4) & \mathrm{C}(3) & \mathrm{C}(2) & 120.3(7) & \mathrm{C}(5) & \mathrm{C}(4) & \mathrm{C}(3) & 118.7(7) \\ \mathrm{C}(6) & \mathrm{C}(5) & \mathrm{C}(4) & 122.8(6) & \mathrm{C}(7) & \mathrm{C}(6) & \mathrm{C}(1) & 121.8(5) \\ \mathrm{C}(7) & \mathrm{C}(6) & \mathrm{C}(5) & 122.9(5) & \mathrm{C}(1) & \mathrm{C}(6) & \mathrm{C}(5) & 115.3(6) \\ \mathrm{C}(8) & \mathrm{C}(7) & \mathrm{C}(6) & 114.5(4) & \mathrm{C}(9) & \mathrm{C}(8) & \mathrm{C}(10) & 107.0(5) \\ \mathrm{C}(9) & \mathrm{C}(8) & \mathrm{C}(11) & 114.8(6) & \mathrm{C}(9) & \mathrm{C}(8) & \mathrm{C}(7) & 109.0(5) \\ \mathrm{C}(10) & \mathrm{C}(8) & \mathrm{C}(11) & 105.8(5) & \mathrm{C}(10) & \mathrm{C}(8) & \mathrm{C}(7) & 110.1(5) \\ \mathrm{C}(11) & \mathrm{C}(8) & \mathrm{C}(7) & 110.1(5) & \mathrm{N}(1) & \mathrm{C}(9) & \mathrm{C}(8) & 178.7(7) \\ \mathrm{N}(2) & \mathrm{C}(10) & \mathrm{C}(8) & 178.2(7) & \mathrm{C}(12) & \mathrm{C}(11) & \mathrm{C}(16) & 105.0(8) \\ \mathrm{C}(12) & \mathrm{C}(11) & \mathrm{C}(8) & 118.0(8) & \mathrm{C}(16) & \mathrm{C}(11) & \mathrm{C}(8) & 117.9(6) \\ \mathrm{C}(13) & \mathrm{C}(12) & \mathrm{C}(11) & 105.4(9) & \mathrm{C}(14) & \mathrm{C}(13) & \mathrm{C}(17) & 106.7(9) \\ \mathrm{C}(14) & \mathrm{C}(13) & \mathrm{C}(12) & 106.6(8) & \mathrm{C}(17) & \mathrm{C}(13) & \mathrm{C}(12) & 92.8(9) \\ \mathrm{C}(15) & \mathrm{C}(14) & \mathrm{C}(13) & 101.8(7) & \mathrm{C}(16) & \mathrm{C}(15) & \mathrm{C}(14) & 108.8(8) \\ \mathrm{C}(17) & \mathrm{C}(16) & \mathrm{C}(11) & 91.6(8) & \mathrm{C}(17) & \mathrm{C}(16) & \mathrm{C}(15) & 102.8(9) \\ \mathrm{C}(11) & \mathrm{C}(16) & \mathrm{C}(15) & 102.5(8) & \mathrm{C}(13) & \mathrm{C}(17) & \mathrm{C}(16) & 99.6(9)\end{array}$


Table 7. Bond angles involving hydrogens ( $\left.{ }^{\circ}\right)$

$\begin{array}{llllllll}\text { atom } & \text { atom } & \text { atom } & \text { angle } & \text { atom } & \text { atom } & \text { atom } & \text { angle } \\ \mathrm{C}(2) & \mathrm{C}(1) & \mathrm{H}(1) & 120.3(8) & \mathrm{C}(6) & \mathrm{C}(1) & \mathrm{H}(1) & 118.1(7) \\ \mathrm{C}(3) & \mathrm{C}(2) & \mathrm{H}(2) & 118.6(9) & \mathrm{H}(2) & \mathrm{C}(2) & \mathrm{C}(1) & 120.0(9) \\ \mathrm{C}(4) & \mathrm{C}(3) & \mathrm{H}(3) & 119.9(9) & \mathrm{H}(3) & \mathrm{C}(3) & \mathrm{C}(2) & 119.8(9) \\ \mathrm{C}(5) & \mathrm{C}(4) & \mathrm{H}(4) & 122.4(9) & \mathrm{H}(4) & \mathrm{C}(4) & \mathrm{C}(3) & 118.8(9) \\ \mathrm{C}(6) & \mathrm{C}(5) & \mathrm{H}(5) & 117.2(7) & \mathrm{H}(5) & \mathrm{C}(5) & \mathrm{C}(4) & 120.0(8) \\ \mathrm{C}(8) & \mathrm{C}(7) & \mathrm{H}(6) & 106.7(6) & \mathrm{C}(8) & \mathrm{C}(7) & \mathrm{H}(7) & 109.6(6) \\ \mathrm{H}(6) & \mathrm{C}(7) & \mathrm{H}(7) & 109.5(7) & \mathrm{H}(6) & \mathrm{C}(7) & \mathrm{C}(6) & 106.5(6) \\ \mathrm{H}(7) & \mathrm{C}(7) & \mathrm{C}(6) & 109.9(6) & \mathrm{C}(12) & \mathrm{C}(11) & \mathrm{H}(8) & 105.5(9) \\ \mathrm{C}(16) & \mathrm{C}(11) & \mathrm{H}(8) & 104(1) & \mathrm{H}(8) & \mathrm{C}(11) & \mathrm{C}(8) & 104.7(9) \\ \mathrm{C}(13) & \mathrm{C}(12) & \mathrm{H}(9) & 109(1) & \mathrm{C}(13) & \mathrm{C}(12) & \mathrm{H}(10) & 113(1) \\ \mathrm{H}(9) & \mathrm{C}(12) & \mathrm{H}(10) & 109(1) & \mathrm{H}(9) & \mathrm{C}(12) & \mathrm{C}(11) & 115(1) \\ \mathrm{H}(10) & \mathrm{C}(12) & \mathrm{C}(11) & 102(1) & \mathrm{C}(14) & \mathrm{C}(13) & \mathrm{H}(11) & 114(1) \\ \mathrm{C}(17) & \mathrm{C}(13) & \mathrm{H}(11) & 117(1) & \mathrm{H}(11) & \mathrm{C}(13) & \mathrm{C}(12) & 116(1) \\ \mathrm{C}(15) & \mathrm{C}(14) & \mathrm{H}(12) & 111(1) & \mathrm{C}(15) & \mathrm{C}(14) & \mathrm{H}(13) & 109.3(9) \\ \mathrm{H}(12) & \mathrm{C}(14) & \mathrm{H}(13) & 109(1) & \mathrm{H}(12) & \mathrm{C}(14) & \mathrm{C}(13) & 111.2(9) \\ \mathrm{H}(13) & \mathrm{C}(14) & \mathrm{C}(13) & 113.6(9) & \mathrm{C}(16) & \mathrm{C}(15) & \mathrm{H}(14) & 107(1) \\ \mathrm{C}(16) & \mathrm{C}(15) & \mathrm{H}(15) & 111(1) & \mathrm{H}(14) & \mathrm{C}(15) & \mathrm{H}(15) & 109(1) \\ \mathrm{H}(14) & \mathrm{C}(15) & \mathrm{C}(14) & 109(1) & \mathrm{H}(15) & \mathrm{C}(15) & \mathrm{C}(14) & 111(1) \\ \mathrm{C}(17) & \mathrm{C}(16) & \mathrm{H}(16) & 117(1) & \mathrm{H}(16) & \mathrm{C}(16) & \mathrm{C}(11) & 116(1) \\ \mathrm{H}(16) & \mathrm{C}(16) & \mathrm{C}(15) & 120(1) & \mathrm{H}(17) & \mathrm{C}(17) & \mathrm{H}(18) & 109(1) \\ \mathrm{H}(17) & \mathrm{C}(17) & \mathrm{C}(13) & 115(1) & \mathrm{H}(17) & \mathrm{C}(17) & \mathrm{C}(16) & 115(1) \\ \mathrm{H}(18) & \mathrm{C}(17) & \mathrm{C}(13) & 108(1) & \mathrm{H}(18) & \mathrm{C}(17) & \mathrm{C}(16) & 107(1)\end{array}$


Table 8. Torsion Angles( $\left.{ }^{0}\right)$

atom1 atom2 atom3 atom4 angle

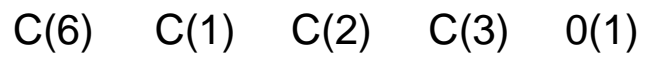

$\begin{array}{lllll}\mathrm{C}(2) & \mathrm{C}(1) & \mathrm{C}(6) & \mathrm{C}(7) & 177.8(5)\end{array}$

$\mathrm{C}(2) \quad \mathrm{C}(3) \quad \mathrm{C}(4) \quad \mathrm{C}(5) \quad-1.4(9)$

C(4) $\quad \mathrm{C}(5) \quad \mathrm{C}(6) \quad \mathrm{C}(1) \quad-1.1(8)$

$\begin{array}{lllll}\mathrm{C}(1) & \mathrm{C}(6) & \mathrm{C}(7) & \mathrm{C}(8) & 91.4(6)\end{array}$

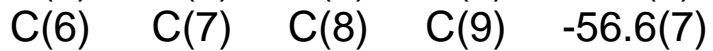

$\mathrm{C}(6) \quad \mathrm{C}(7) \quad \mathrm{C}(8) \quad \mathrm{C}(11) \quad 176.6(5)$

$\begin{array}{lllll}C(7) & C(8) & C(11) & C(16) & -174.4(6)\end{array}$

C(9) $\quad \mathrm{C}(8) \quad \mathrm{C}(11) \quad \mathrm{C}(16) \quad 62.2(9)$

C(10) $\quad C(8) \quad C(11) \quad C(16) \quad-55.5(8)$

C(16) C(11) C(12) C(13) 3(1)

$\begin{array}{lllll}C(8) & C(11) & C(16) & C(17) & -100.5(8)\end{array}$

C(12) C(11) C(16) C(17) 33.3(9)

$\begin{array}{lllll}C(11) & C(12) & C(13) & C(17) & -39(1)\end{array}$

C(17) C(13) C(14) C(15) 28(1)

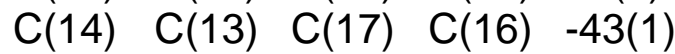

C(14) C(15) C(16) C(11) 69(1)

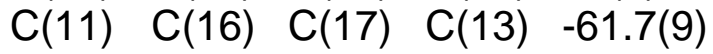

atom1 atom2 atom3 atom4 angle

$\begin{array}{lllll}\mathrm{C}(2) & \mathrm{C}(1) & \mathrm{C}(6) & \mathrm{C}(5) & 0.2(8)\end{array}$

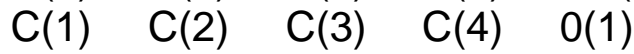

$\begin{array}{lllll}\mathrm{C}(3) & \mathrm{C}(4) & \mathrm{C}(5) & \mathrm{C}(6) & 1.7(9)\end{array}$

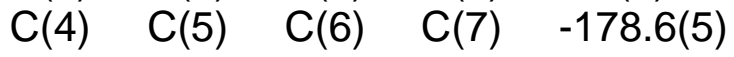

$\begin{array}{lllll}C(5) & C(6) & C(7) & C(8) & -91.3(6)\end{array}$

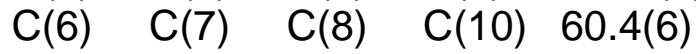

$\begin{array}{lllll}\mathrm{C}(7) & \mathrm{C}(8) & \mathrm{C}(11) & \mathrm{C}(12) & 57.8(9)\end{array}$

$\begin{array}{lllll}\mathrm{C}(9) & \mathrm{C}(8) & \mathrm{C}(11) & \mathrm{C}(12) & -65.6(9)\end{array}$

$\begin{array}{lllll}C(10) & C(8) & C(11) & C(12) & 176.7(8)\end{array}$

$\begin{array}{lllll}\mathrm{C}(8) & \mathrm{C}(11) & \mathrm{C}(12) & \mathrm{C}(13) & 136.8(8)\end{array}$

C(8) C(11) C(16) C(15) 156.0(9)

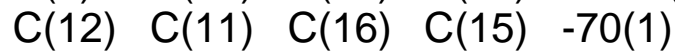

$\mathrm{C}(11) \quad \mathrm{C}(12) \quad \mathrm{C}(13) \quad \mathrm{C}(14) \quad 68(1)$

C(12) C(13) C(14) C(15) -69(1)

C(12) C(13) C(17) C(16) 64.3(9)

C(13) $\quad C(14) \quad C(15) \quad C(16)-1(1)$

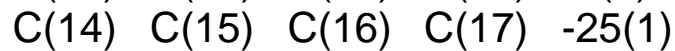

C(15) C(16) C(17) C(13) 41(1)

The sign is positive if when looking from atom 2 to atom 3 a clock-wise motion of atom 1 would superimpose it on atom 4. 
Table 9. Torsion Angles involving hydrogens( $\left({ }^{0}\right)$

\begin{tabular}{|c|c|c|c|c|c|c|c|c|c|}
\hline & & & & & 0 & $n$ & & & \\
\hline$C(6)$ & $C(1)$ & $C(2)$ & $\mathrm{H}(2)$ & 178.3(8) & $H(1)$ & $C(1)$ & $C(2)$ & $C(3)$ & $175.3(7)$ \\
\hline$H(1)$ & $C(1)$ & $C(2)$ & $H(2)$ & $-6(1)$ & $H(1)$ & $C(1)$ & $C(6)$ & C(5) & $-175.2(7)$ \\
\hline$H(1)$ & $C(1)$ & $C(6)$ & $C(7)$ & $2.3(9)$ & $C(1)$ & $C(2)$ & $C(3)$ & $H(3)$ & $179.4(8)$ \\
\hline $\mathrm{H}(2)$ & $C(2)$ & $C(3)$ & $C(4)$ & $-177.7(8)$ & $H(2)$ & $C(2)$ & $C(3)$ & $H(3)$ & $1(1)$ \\
\hline$C(2)$ & $C(3)$ & $C(4)$ & $\mathrm{H}(4)$ & $-179.4(7)$ & $H(3)$ & $C(3)$ & $C(4)$ & $C(5)$ & $179(508)$ \\
\hline $\mathrm{H}(3)$ & $C(3)$ & $C(4)$ & $\mathrm{H}(4)$ & $1(1)$ & $C(3)$ & $C(4)$ & $C(5)$ & $H(5)$ & $-178.8(7)$ \\
\hline $\mathrm{H}(4)$ & $\mathrm{C}(4)$ & $C(5)$ & $C(6)$ & $179(359)$ & $H(4)$ & $C(4)$ & $C(5)$ & $H(5)$ & $0(1)$ \\
\hline$H(5)$ & $C(5)$ & $C(6)$ & $C(1)$ & $179.4(6)$ & $H(5)$ & $C(5)$ & $C(6)$ & $C(7)$ & $1.8(9)$ \\
\hline$C(1)$ & $C(6)$ & $C(7)$ & $H(6)$ & $-151.0(6)$ & $C(1)$ & $C(6)$ & $C(7)$ & $H(7)$ & $-32.5(8)$ \\
\hline$C(5)$ & $C(6)$ & $C(7)$ & $H(6)$ & $26.4(8)$ & $C(5)$ & $C(6)$ & $C(7)$ & $\mathrm{H}(7)$ & $144.8(6)$ \\
\hline$H(6)$ & $\mathrm{C}(7)$ & $C(8)$ & $\mathrm{C}(9)$ & $-174.2(6)$ & $H(6)$ & $C(7)$ & $C(8)$ & $C(10)$ & $-57.2(7)$ \\
\hline $\mathrm{H}(6)$ & $C(7)$ & $C(8)$ & $C(11)$ & $59.0(8)$ & $\mathrm{H}(7)$ & $C(7)$ & $C(8)$ & $C(9)$ & $67.3(7)$ \\
\hline $\mathrm{H}(7)$ & $\mathrm{C}(7)$ & $C(8)$ & $C(10)$ & $-175.6(6)$ & $\mathrm{H}(7)$ & $C(7)$ & $C(8)$ & $C(11)$ & $-59.4(8)$ \\
\hline$C(7)$ & $C(8)$ & $C(11)$ & $H(8)$ & $-59.0(9)$ & $C(9)$ & $C(8)$ & $C(11)$ & $H(8)$ & 177.6(8) \\
\hline$C(10)$ & $C(8)$ & $C(11)$ & $H(8)$ & 59.9(9) & $C(8)$ & $C(11)$ & $C(12)$ & $H(9)$ & $-101(1)$ \\
\hline$C(8)$ & $C(11)$ & $C(12)$ & $H(10)$ & $17(1)$ & $C(16)$ & $C(11)$ & $C(12)$ & $H(9)$ & $124(1)$ \\
\hline$C(16)$ & $C(11)$ & $C(12)$ & $H(10)$ & $-116(1)$ & $H(8)$ & $C(11)$ & $C(12)$ & $C(13)$ & $-106(1)$ \\
\hline$H(8)$ & $C(11)$ & $C(12)$ & $H(9)$ & $14(1)$ & $H(8)$ & $C(11)$ & $C(12)$ & $H(10)$ & $133(1)$ \\
\hline$C(8)$ & $C(11)$ & $C(16)$ & $H(16)$ & $21(1)$ & $C(12)$ & $C(11)$ & $C(16)$ & $\mathrm{H}(16)$ & 155(1) \\
\hline$H(8)$ & $C(11)$ & $C(16)$ & $C(15)$ & $40(1)$ & $H(8)$ & $C(11)$ & $C(16)$ & $C(17)$ & $144.0(9)$ \\
\hline$H(8)$ & $C(11)$ & $C(16)$ & $H(16)$ & $-94(1)$ & $C(11)$ & C(12) & $C(13)$ & $H(11)$ & $-162(1)$ \\
\hline$H(9)$ & $C(12)$ & $C(13)$ & $C(14)$ & $-56(1)$ & $\mathrm{H}(9)$ & $C(12)$ & $C(13)$ & $\mathrm{C}(17)$ & $-164(1)$ \\
\hline$H(9)$ & $C(12)$ & $C(13)$ & $H(11)$ & $72(1)$ & $H(10)$ & $C(12)$ & $C(13)$ & $C(14)$ & $-179(359)$ \\
\hline $\mathrm{H}(10)$ & $C(12)$ & $C(13)$ & $C(17)$ & $72(1)$ & $H(10)$ & $C(12)$ & $C(13)$ & $H(11)$ & $-50(1)$ \\
\hline$C(12)$ & $C(13)$ & $C(14)$ & $H(12)$ & $49(1)$ & $C(12)$ & $C(13)$ & $C(14)$ & $H(13)$ & 173.1(9) \\
\hline$C(17)$ & $C(13)$ & $C(14)$ & $H(12)$ & $147(1)$ & $C(17)$ & $C(13)$ & $C(14)$ & $H(13)$ & $-88(1)$ \\
\hline$H(11)$ & $C(13)$ & $C(14)$ & $C(15)$ & $160(1)$ & $H(11)$ & $C(13)$ & $C(14)$ & $H(12)$ & $-80(1)$ \\
\hline$H(11)$ & $C(13)$ & $C(14)$ & $H(13)$ & $43(1)$ & $C(12)$ & $C(13)$ & $C(17)$ & $\mathrm{H}(17)$ & $-171(1)$ \\
\hline$C(12)$ & $C(13)$ & $C(17)$ & $H(18)$ & $-48(1)$ & $C(14)$ & $C(13)$ & $C(17)$ & $\mathrm{H}(17)$ & $79(1)$ \\
\hline$C(14)$ & $C(13)$ & $C(17)$ & $H(18)$ & $-156(1)$ & $H(11)$ & $C(13)$ & $C(17)$ & $C(16)$ & $-174(1)$ \\
\hline$H(11)$ & $C(13)$ & $C(17)$ & $\mathrm{H}(17)$ & $-50(1)$ & $\mathrm{H}(11)$ & $C(13)$ & $C(17)$ & $\mathrm{H}(18)$ & 72(1) \\
\hline$C(13)$ & $C(14)$ & $C(15)$ & $H(14)$ & 115(1) & $C(13)$ & $C(14)$ & $C(15)$ & $\mathrm{H}(15)$ & $-123(1)$ \\
\hline$H(12)$ & $C(14)$ & $C(15)$ & $C(16)$ & $-119(1)$ & $H(12)$ & $C(14)$ & $C(15)$ & $H(14)$ & $-2(1)$ \\
\hline$H(12)$ & $C(14)$ & $C(15)$ & $H(15)$ & 117(1) & $H(13)$ & $C(14)$ & $C(15)$ & $C(16)$ & $119(1)$ \\
\hline$H(13)$ & $C(14)$ & $C(15)$ & $H(14)$ & $-123(1)$ & $H(13)$ & $C(14)$ & $C(15)$ & $\mathrm{H}(15)$ & $-3(1)$ \\
\hline$C(14)$ & $C(15)$ & $C(16)$ & $H(16)$ & $-158(1)$ & $H(14)$ & $C(15)$ & $C(16)$ & $C(11)$ & $-48(1)$ \\
\hline$H(14)$ & $C(15)$ & $C(16)$ & $C(17)$ & $-143(1)$ & $H(14)$ & $C(15)$ & $C(16)$ & $H(16)$ & $83(1)$ \\
\hline
\end{tabular}


Table 9. Torsion angles involving hydrogens (0) -- continued

atom1 atom2 atom3 atom4 angle atom1 atom2 atom3 atom4 angle

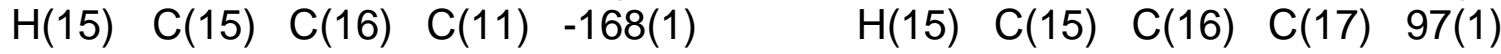

$\begin{array}{llllllll}\mathrm{H}(15) & \mathrm{C}(15) & \mathrm{C}(16) & \mathrm{H}(16) & -35(2) & \mathrm{C}(11) & \mathrm{C}(16) & \mathrm{C}(17) \\ \mathrm{H}(17) & 174(1)\end{array}$

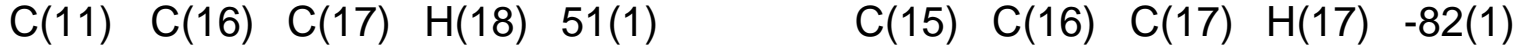

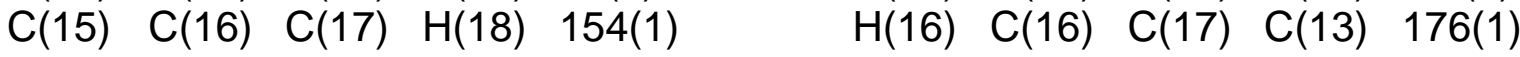

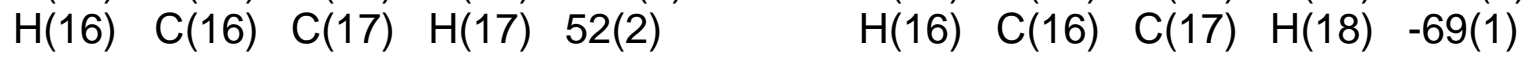

The sign is positive if when looking from atom 2 to atom 3 a clock-wise motion of atom 1 would superimpose it on atom 4 . 


\section{X-ray crystallographic Data of endo-6e}

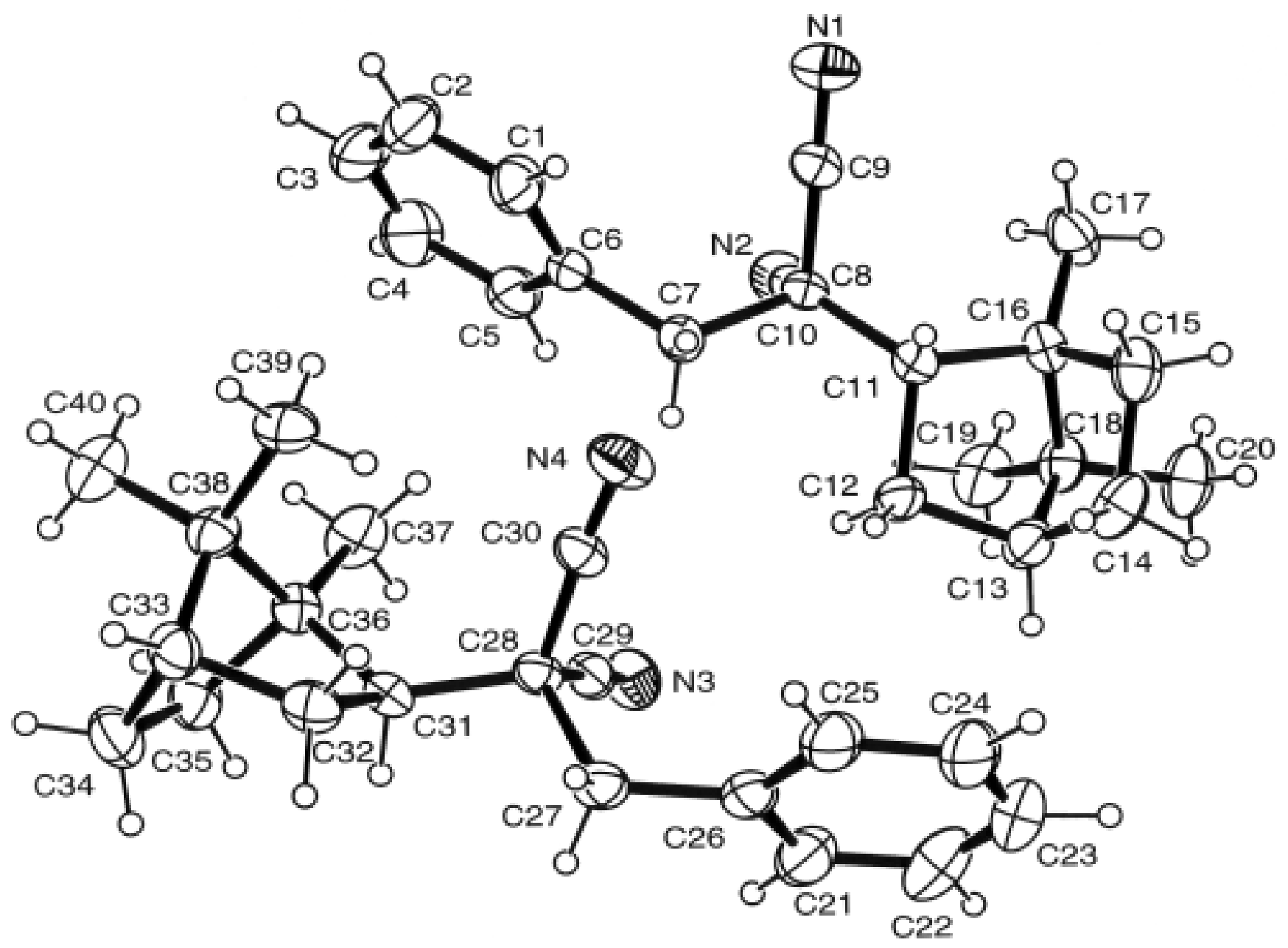

Figure S6. ORTEP drawing of endo-6e (ellipsoids at 30\% probability). 


\section{Experimental}

\section{$\underline{\text { Data Collection }}$}

A colorless prism crystal of $\mathrm{C}_{20} \mathrm{H}_{24} \mathrm{~N}_{2}$ having approximate dimensions of $0.75 \mathrm{x}$ $0.50 \times 0.25 \mathrm{~mm}$ was mounted on a glass fiber. All measurements were made on a Rigaku RAXIS RAPID imaging plate area detector with graphite monochromated Mo-Ka radiation.

Indexing was performed from 30 oscillations that were exposed for 180 seconds. The crystal-to-detector distance was $127.40 \mathrm{~mm}$.

Cell constants and an orientation matrix for data collection corresponded to a C-centered triclinic cell with dimensions:

$$
\begin{array}{lll}
\mathrm{a}= & 11.7618(7) \AA & \alpha=92.734(3)^{\mathrm{O}} \\
\mathrm{b}=13.1850(8) \AA & \beta=112.610(2)^{\mathrm{O}} \\
\mathrm{C}=13.7331(8) \AA & \gamma=114.056(3)^{\mathrm{O}} \\
\mathrm{V}=1741.7(2) \AA^{3} &
\end{array}
$$

For $Z=4$ and F.W. = 292.42, the calculated density is $1.12 \mathrm{~g} / \mathrm{cm}^{3}$. Based on the systematic absences of:

$h k l: \quad h+k \pm 2 n$

packing considerations, a statistical analysis of intensity distribution, and the successful solution and refinement of the structure, the space group was determined to be:

\section{C1 (\#1)}

The data were collected at a temperature of $23 \pm 1^{\circ} \mathrm{C}$ to a maximum $2 \theta$ value of 54.90. A total of 44 oscillation images were collected. A sweep of data was done using $\omega$ scans from 130.0 to $190.0^{\circ}$ in $5.0^{\circ} \mathrm{step}$, at $\chi=45.0^{\circ}$ and $\phi=0.0^{\circ}$. The exposure rate was 289.8 [sec./0]. A second sweep was performed using $\omega$ scans from 0.0 to $160.0^{\circ}$ in 5.00 step, at $\chi=45.00$ and $\phi=180.00$. The exposure rate was 289.8 [sec./0]. The 
crystal-to-detector distance was $127.40 \mathrm{~mm}$. Readout was performed in the $0.100 \mathrm{~mm}$ pixel mode.

\section{Data Reduction}

A total of 7730 reflections was collected.

The linear absorption coefficient, $\mu$, for Mo-K $\alpha$ radiation is $0.7 \mathrm{~cm}^{-1}$. An empirical absorption correction was applied which resulted in transmission factors ranging from 0.91 to 1.13 . The data were corrected for Lorentz and polarization effects.

\section{$\underline{\text { Structure Solution and Refinement }}$}

The structure was solved by direct methods ${ }^{1}$ and expanded using Fourier techniques $^{2}$. The non-hydrogen atoms were refined anisotropically. Hydrogen atoms were refined using the riding model. The final cycle of full-matrix least-squares refinement ${ }^{3}$ on $\mathrm{F}^{2}$ was based on 3717 observed reflections and 445 variable parameters and converged (largest parameter shift was 0.00 times its esd) with unweighted and weighted agreement factors of:

$$
\begin{gathered}
\mathrm{R} 1=\Sigma\|\mathrm{Fo}|-| \mathrm{Fc}\| / \Sigma|\mathrm{Fo}|=0.061 \\
\mathrm{wR} 2=\left[\Sigma\left(\mathrm{w}\left(\mathrm{Fo}^{2}-\mathrm{Fc}^{2}\right)^{2}\right) / \Sigma \mathrm{w}\left(\mathrm{Fo}^{2}\right)^{2}\right]^{1 / 2}=0.132
\end{gathered}
$$

The standard deviation of an observation of unit weight ${ }^{4}$ was 1.06. A Sheldrick weighting scheme was used. Plots of $\Sigma \mathrm{w}(|\mathrm{Fo}|-|\mathrm{Fc}|)^{2}$ versus $|\mathrm{Fo}|$, reflection order in data collection, $\sin \theta / \lambda$ and various classes of indices showed no unusual trends. The maximum and minimum peaks on the final difference Fourier map corresponded to 0.56 and $-0.44 \mathrm{e}^{-} / \AA^{3}$, respectively.

Neutral atom scattering factors were taken from Cromer and Waber 5 . Anomalous dispersion effects were included in Fcalc6; the values for $\Delta f^{\prime}$ and $\Delta f^{\prime \prime}$ were those of Creagh and McAuley 7 . The values for the mass attenuation coefficients are those of Creagh and Hubbell 8 . All calculations were performed using the CrystalStructure 9,10 crystallographic software package. 
References

(1) SIR92: Altomare, A., Cascarano, G., Giacovazzo, C., Guagliardi, A., Burla, M., Polidori, G., and Camalli, M. (1994) J. Appl. Cryst., 27, 435.

(2) DIRDIF99: Beurskens, P.T., Admiraal, G., Beurskens, G., Bosman, W.P., de Gelder, R., Israel, R. and Smits, J.M.M.(1999). The DIRDIF-99 program system, Technical Report of the Crystallography Laboratory, University of Nijmegen, The Netherlands.

(3) Least Squares function minimized:

$$
\Sigma w\left(\mathrm{~F}_{\mathrm{o}}{ }^{2}-\mathrm{F}_{\mathrm{c}}{ }^{2}\right)^{2} \quad \text { where } \mathrm{w}=\text { Least Squares weights. }
$$

(4) Standard deviation of an observation of unit weight:

$$
\begin{aligned}
& {\left[\Sigma w\left(\mathrm{~F}_{\mathrm{O}}{ }^{2}-\mathrm{F}_{\mathrm{C}}{ }^{2}\right)^{2} /\left(\mathrm{N}_{\mathrm{O}}-\mathrm{N}_{\mathrm{v}}\right)\right]^{1 / 2}} \\
& \text { where: } \quad \mathrm{N}_{\mathrm{O}}=\text { number of observations } \\
& \mathrm{N}_{\mathrm{V}}=\text { number of variables }
\end{aligned}
$$

(5) Cromer, D. T. \& Waber, J. T.; "International Tables for X-ray Crystallography", Vol. IV, The Kynoch Press, Birmingham, England, Table 2.2 A (1974).

(6) Ibers, J. A. \& Hamilton, W. C.; Acta Crystallogr., 17, 781 (1964).

(7) Creagh, D. C. \& McAuley, W.J .; "International Tables for Crystallography", Vol C, (A.J.C. Wilson, ed.), Kluwer Academic Publishers, Boston, Table 4.2.6.8, pages 219-222 (1992).

(8) Creagh, D. C. \& Hubbell, J.H..; "International Tables for Crystallography", Vol C, (A.J.C. Wilson, ed.), Kluwer Academic Publishers, Boston, Table 4.2.4.3, pages 200-206 (1992).

(9) CrystalStructure 3.5.1: Crystal Structure Analysis Package, Rigaku and Rigaku/MSC (2000-2003). 9009 New Trails Dr. The Woodlands TX 77381 USA.

(10) CRYSTALS Issue 10: Watkin, D.J., Prout, C.K. Carruthers, J.R. \& Betteridge, P.W. Chemical Crystallography Laboratory, Oxford, UK. (1996) 
EXPERIMENTAL DETAILS

\author{
A. Crystal Data
}

Empirical Formula

Formula Weight

Crystal Color, Habit

Crystal Dimensions

Crystal System

Lattice Type

Indexing Images

Detector Position

Pixel Size

Lattice Parameters

Space Group

$Z$ value

$D_{\text {calc }}$
$\mathrm{C}_{20} \mathrm{H}_{24} \mathrm{~N}_{2}$

292.42

colorless, prism

$0.75 \times 0.50 \times 0.25 \mathrm{~mm}$

triclinic

C-centered

3 oscillations @ 180.0 seconds

$127.40 \mathrm{~mm}$

$0.100 \mathrm{~mm}$

$$
\begin{aligned}
& \mathrm{a}=11.7618(7) \AA \\
& \mathrm{b}=13.1850(8) \AA \\
& \mathrm{c}=13.7331(8) \AA \\
& \alpha=92.734(3) \circ \\
& \beta=112.610(2) \circ \\
& \gamma=114.056(3) \circ \\
& \mathrm{V}=1741.7(2) \AA^{3}
\end{aligned}
$$

C1 (\#1)

4

$1.115 \mathrm{~g} / \mathrm{cm}^{3}$ 
$\mu(\operatorname{MoK} \alpha)$ $0.65 \mathrm{~cm}^{-1}$ 
B. Intensity Measurements

Diffractometer

Radiation

Detector Aperture

Data Images

$\omega$ oscillation Range $(\chi=45.0, \phi=0.0)$

Exposure Rate

$\omega$ oscillation Range ( $\chi=45.0, \phi=180.0)$

Exposure Rate

Detector Position

Pixel Size

$2 \theta_{\max }$

No. of Reflections Measured

Corrections
Rigaku RAXIS-RAPID

$\operatorname{MoK} \alpha(\lambda=0.71069 \AA)$

graphite monochromated

$270 \mathrm{~mm} \times 256 \mathrm{~mm}$

44 exposures

$130.0-190.00$

$289.8 \mathrm{sec} . / 0$

$0.0-160.00$

$289.8 \mathrm{sec} . / 0$

$127.40 \mathrm{~mm}$

$0.100 \mathrm{~mm}$

54.90

Total: 7730

Lorentz-polarization

Absorption

(trans. factors: $0.9064-1.1311$ ) 
C. Structure Solution and Refinement

Structure Solution

Refinement

Function Minimized

Least Squares Weights

Fo2)

Anomalous Dispersion

No. Observations $(\mathrm{I}>1.00 \sigma(\mathrm{I}))$

No. Variables

Reflection/Parameter Ratio

Residuals: $\mathrm{R}(\mathrm{I}>1.00 \sigma(\mathrm{I}))$

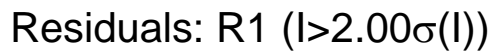

Residuals: wR2 $(\mathrm{I}>1.00 \sigma(\mathrm{I}))$

Goodness of Fit Indicator

Max Shift/Error in Final Cycle

Maximum peak in Final Diff. Map

Minimum peak in Final Diff. Map
Direct Methods (SIR92)

Full-matrix least-squares on $\mathrm{F}^{2}$

$\Sigma \mathrm{w}\left(\mathrm{Fo}^{2}-\mathrm{Fc}^{2}\right)^{2}$

$1 /\left[0.0010 \mathrm{Fo}^{2}+1.0000 \sigma\left(\mathrm{Fo}^{2}\right)+0.2000\right] /(4$

All non-hydrogen atoms

3717

445

8.35

0.063

0.061

0.132

1.059

0.000

$0.56 \mathrm{e}^{-/} \AA^{3}$

$-0.44 \mathrm{e}^{-/} / \AA^{3}$ 
Table 1. Atomic coordinates and $\mathrm{B}_{\mathrm{iso}} / \mathrm{B}_{\mathrm{eq}}$

$\begin{array}{lrrrl}\text { atom } & x & y & z & B_{\text {eq }} \\ \mathrm{N}(3) & 0.5260 & 0.1798 & 1.0270 & 5.318 \\ \mathrm{~N}(4) & 0.3400 & -0.1733 & 0.9992 & 5.292 \\ \mathrm{~N}(1) & -0.1059 & -0.1227 & 1.0410 & 5.912 \\ \mathrm{~N}(2) & 0.0613 & 0.2169 & 1.0246 & 5.862 \\ \mathrm{C}(10) & 0.0636 & 0.1326 & 1.0072 & 3.943 \\ \mathrm{C}(8) & 0.0706 & 0.0257 & 0.9843 & 3.250 \\ \mathrm{C}(30) & 0.4429 & -0.0958 & 1.0201 & 3.820 \\ \mathrm{C}(29) & 0.5496 & 0.1052 & 1.0376 & 3.802 \\ \mathrm{C}(28) & 0.5763 & 0.0045 & 1.0434 & 3.290 \\ \mathrm{C}(11) & 0.0483 & 0.0020 & 0.8628 & 3.292 \\ \mathrm{C}(37) & 0.6860 & 0.0241 & 1.2645 & 3.603 \\ \mathrm{C}(7) & 0.2198 & 0.0452 & 1.0634 & 4.064 \\ \mathrm{C}(26) & 0.5070 & -0.0426 & 0.8351 & 4.492 \\ \mathrm{C}(38) & 0.7964 & 0.1499 & 1.3293 & 3.892 \\ \mathrm{C}(6) & 0.2645 & 0.0838 & 1.1831 & 4.002 \\ \mathrm{C}(31) & 0.6934 & 0.0132 & 1.1521 & 3.245 \\ \mathrm{C}(9) & -0.0313 & -0.0613 & 1.0132 & 4.065 \\ \mathrm{C}(12) & 0.1002 & -0.0798 & 0.8327 & 4.365 \\ \mathrm{C}(32) & 0.8414 & 0.1090 & 1.1806 & 4.223 \\ \mathrm{C}(33) & 0.9103 & 0.1427 & 1.3053 & 4.342 \\ \mathrm{C}(18) & -0.1427 & -0.1701 & 0.7199 & 4.410 \\ \mathrm{C}(17) & -0.0993 & -0.0418 & 0.7648 & 3.844 \\ \mathrm{C}(27) & 0.6185 & -0.0114 & 0.9497 & 4.252 \\ \mathrm{C}(13) & -0.0067 & -0.1429 & 0.7151 & 4.640 \\ \mathrm{C}(1) & 0.3465 & 0.1994 & 1.2365 & 5.635 \\ \mathrm{C}(39) & 0.7582 & 0.2431 & 1.2906 & 4.898 \\ \mathrm{C}(25) & 0.4108 & -0.1552 & 0.7825 & 6.032 \\ \mathrm{C}(35) & 0.7647 & -0.0406 & 1.3246 & 5.263 \\ \mathrm{C}(36) & 0.5455 & -0.0156 & 1.2623 & 5.343 \\ \mathrm{C}(14) & 0.0050 & -0.0545 & 0.6458 & 5.695 \\ \mathrm{C}(19) & -0.1749 & -0.2493 & 0.7928 & 6.049 \\ \mathrm{C}(5) & 0.2306 & 0.0047 & 1.2431 & 5.556 \\ \mathrm{C}(16) & -0.2041 & -0.0155 & 0.7824 & 5.598 \\ \mathrm{C}(15) & -0.0653 & 0.0102 & 0.6748 & 5.130 \\ \mathrm{C}(21) & 0.4987 & 0.0394 & 0.7796 & 5.958 \\ \mathrm{C}(40) & 0.8336 & 0.1715 & 1.4506 & 6.063 \\ \mathrm{C}(34) & 0.9160 & 0.0370 & 1.3449 & 5.911\end{array}$


Table 1. Atomic coordinates and $\mathrm{B}_{\mathrm{iso}} / \mathrm{B}_{\mathrm{eq}}$ (continued)

$\begin{array}{lcccl}\text { atom } & x & y & z & B_{\text {eq }} \\ \mathrm{C}(20) & -0.2702 & -0.2248 & 0.6086 & 6.739 \\ \mathrm{C}(4) & 0.2786 & 0.0425 & 1.3542 & 7.584 \\ \mathrm{C}(23) & 0.3038 & -0.1019 & 0.6245 & 8.118 \\ \mathrm{C}(2) & 0.3907 & 0.2341 & 1.3464 & 7.757 \\ \mathrm{C}(3) & 0.3570 & 0.1554 & 1.4039 & 7.747 \\ \mathrm{C}(22) & 0.3965 & 0.0082 & 0.6731 & 8.113 \\ \mathrm{C}(24) & 0.3089 & -0.1828 & 0.6769 & 8.272 \\ \mathrm{H}(1) & 0.3696 & 0.2533 & 1.1951 & 6.573 \\ \mathrm{H}(2) & 0.4477 & 0.3130 & 1.3824 & 8.738 \\ \mathrm{H}(3) & 0.3901 & 0.1791 & 1.4801 & 9.967 \\ \mathrm{H}(4) & 0.2496 & -0.0112 & 1.3941 & 10.114 \\ \mathrm{H}(5) & 0.1796 & -0.0745 & 1.2076 & 7.154 \\ \mathrm{H}(6) & 0.2227 & -0.0252 & 1.0522 & 5.053 \\ \mathrm{H}(7) & 0.2847 & 0.1013 & 1.0447 & 5.050 \\ \mathrm{H}(8) & 0.1038 & 0.0741 & 0.8547 & 3.931 \\ \mathrm{H}(9) & 0.1901 & -0.0385 & 0.8365 & 5.150 \\ \mathrm{H}(10) & 0.1020 & -0.1306 & 0.8794 & 5.147 \\ \mathrm{H}(11) & -0.0020 & -0.2082 & 0.6883 & 5.297 \\ \mathrm{H}(12) & 0.1000 & -0.0047 & 0.6661 & 6.377 \\ \mathrm{H}(13) & -0.0411 & -0.0897 & 0.5703 & 6.383 \\ \mathrm{H}(14) & -0.1466 & -0.0011 & 0.6132 & 5.795 \\ \mathrm{H}(15) & -0.0034 & 0.0898 & 0.7027 & 5.795 \\ \mathrm{H}(16) & -0.1671 & 0.0650 & 0.8053 & 7.112 \\ \mathrm{H}(17) & -0.2878 & -0.0445 & 0.7171 & 7.113 \\ \mathrm{H}(18) & -0.2225 & -0.0504 & 0.8373 & 7.114 \\ \mathrm{H}(19) & -0.2563 & -0.2568 & 0.7975 & 6.204 \\ \mathrm{H}(20) & -0.1884 & -0.3224 & 0.7639 & 6.205 \\ \mathrm{H}(21) & -0.0987 & -0.2177 & 0.8632 & 6.198 \\ \mathrm{H}(22) & -0.3504 & -0.2322 & 0.6156 & 6.444 \\ \mathrm{H}(23) & -0.2554 & -0.1783 & 0.5596 & 6.441 \\ \mathrm{H}(24) & -0.2834 & -0.2984 & 0.5816 & 6.444 \\ \mathrm{H}(29) & 0.4169 & -0.2136 & 0.8190 & 7.347 \\ \mathrm{H}(28) & 0.2425 & -0.2600 & 0.6399 & 8.756 \\ \mathrm{H}(27) & 0.2305 & -0.1199 & 0.5542 & 9.644 \\ \mathrm{H}(26) & 0.3958 & 0.0648 & 0.6329 & 10.792 \\ \mathrm{H}(25) & 0.5629 & 0.1175 & 0.8149 & 7.872 \\ \mathrm{H}(30) & 0.6956 & 0.0580 & 0.9588 & 5.728\end{array}$


Table 1. Atomic coordinates and $\mathrm{B}_{\mathrm{iso}} / \mathrm{B}_{\text {eq }}$ (continued)

$\begin{array}{lcccl}\text { atom } & x & y & z & B_{\text {eq }} \\ \mathrm{H}(31) & 0.6449 & -0.0709 & 0.9573 & 5.729 \\ \mathrm{H}(32) & 0.6977 & -0.0566 & 1.1409 & 4.058 \\ \mathrm{H}(33) & 0.8898 & 0.0814 & 1.1543 & 5.279 \\ \mathrm{H}(34) & 0.8361 & 0.1715 & 1.1510 & 5.274 \\ \mathrm{H}(35) & 0.9966 & 0.2106 & 1.3375 & 4.716 \\ \mathrm{H}(36) & 0.9427 & 0.0001 & 1.3030 & 6.880 \\ \mathrm{H}(37) & 0.9785 & 0.0563 & 1.4197 & 6.880 \\ \mathrm{H}(38) & 0.7592 & -0.0483 & 1.3913 & 5.932 \\ \mathrm{H}(39) & 0.7281 & -0.1143 & 1.2801 & 5.932 \\ \mathrm{H}(40) & 0.5547 & -0.0058 & 1.3344 & 6.225 \\ \mathrm{H}(41) & 0.5032 & 0.0278 & 1.2248 & 6.223 \\ \mathrm{H}(42) & 0.4895 & -0.0943 & 1.2255 & 6.223 \\ \mathrm{H}(43) & 0.8351 & 0.3164 & 1.3289 & 6.065 \\ \mathrm{H}(44) & 0.7334 & 0.2332 & 1.2150 & 6.062 \\ \mathrm{H}(45) & 0.6817 & 0.2371 & 1.3027 & 6.065 \\ \mathrm{H}(46) & 0.8584 & 0.1156 & 1.4797 & 6.648 \\ \mathrm{H}(47) & 0.9100 & 0.2456 & 1.4866 & 6.650 \\ \mathrm{H}(48) & 0.7565 & 0.1671 & 1.4610 & 6.650\end{array}$

$B_{\text {eq }}=8 / 3 \pi^{2}\left(U_{11}\left(a a^{\star}\right)^{2}+U_{22}\left(b b^{\star}\right)^{2}+U_{33}\left(c c^{\star}\right)^{2}+2 U_{12}\left(a a^{*} b b^{*}\right) \cos \gamma+2 U_{13}\left(a a^{*} c c^{\star}\right) \cos \beta+2 U_{23}\left(b b^{*} c c^{\star}\right) \cos \alpha\right)$ 
Table 2. Anisotropic Displacement Parameters

\begin{tabular}{|c|c|c|c|c|c|c|}
\hline atom & $U_{11}$ & $\mathrm{U}_{22}$ & $\mathrm{U}_{33}$ & $U_{12}$ & $U_{13}$ & $U_{23}$ \\
\hline$N(3)$ & 0.0799 & 0.0753 & 0.0805 & 0.0545 & 0.0454 & 0.0382 \\
\hline $\mathrm{N}(4)$ & 0.0494 & 0.0659 & 0.0755 & 0.0199 & 0.0249 & 0.0189 \\
\hline$N(1)$ & 0.0830 & 0.0822 & 0.0891 & 0.0435 & 0.0580 & 0.0430 \\
\hline$N(2)$ & 0.0839 & 0.0561 & 0.0975 & 0.0403 & 0.0460 & 0.0163 \\
\hline$C(10)$ & 0.0503 & 0.0511 & 0.0578 & 0.0272 & 0.0284 & 0.0174 \\
\hline$C(8)$ & 0.0410 & 0.0431 & 0.0474 & 0.0232 & 0.0231 & 0.0113 \\
\hline$C(30)$ & 0.0479 & 0.0555 & 0.0489 & 0.0300 & 0.0214 & 0.0155 \\
\hline$C(29)$ & 0.0515 & 0.0572 & 0.0551 & 0.0332 & 0.0318 & 0.0266 \\
\hline C(28) & 0.0457 & 0.0467 & 0.0465 & 0.0271 & 0.0268 & 0.0175 \\
\hline$C(11)$ & 0.0367 & 0.0421 & 0.0457 & 0.0163 & 0.0196 & 0.0113 \\
\hline$C(37)$ & 0.0479 & 0.0393 & 0.0478 & 0.0177 & 0.0214 & 0.0161 \\
\hline$C(7)$ & 0.0461 & 0.0682 & 0.0456 & 0.0331 & 0.0188 & 0.0105 \\
\hline$C(26)$ & 0.0713 & 0.0717 & 0.0497 & 0.0447 & 0.0348 & 0.0184 \\
\hline$C(38)$ & 0.0522 & 0.0414 & 0.0501 & 0.0189 & 0.0217 & 0.0098 \\
\hline$C(6)$ & 0.0607 & 0.0626 & 0.0434 & 0.0423 & 0.0221 & 0.0141 \\
\hline$C(31)$ & 0.0381 & 0.0416 & 0.0488 & 0.0223 & 0.0196 & 0.0149 \\
\hline $\mathrm{C}(9)$ & 0.0565 & 0.0560 & 0.0617 & 0.0355 & 0.0337 & 0.0241 \\
\hline C(12) & 0.0462 & 0.0664 & 0.0504 & 0.0274 & 0.0189 & -0.0004 \\
\hline$C(32)$ & 0.0444 & 0.0564 & 0.0662 & 0.0223 & 0.0317 & 0.0163 \\
\hline$C(33)$ & 0.0401 & 0.0497 & 0.0595 & 0.0150 & 0.0139 & 0.0076 \\
\hline$C(18)$ & 0.0448 & 0.0463 & 0.0500 & 0.0066 & 0.0118 & 0.0075 \\
\hline$C(17)$ & 0.0429 & 0.0543 & 0.0478 & 0.0232 & 0.0175 & 0.0198 \\
\hline$C(27)$ & 0.0599 & 0.0658 & 0.0558 & 0.0378 & 0.0347 & 0.0189 \\
\hline$C(13)$ & 0.0570 & 0.0617 & 0.0490 & 0.0242 & 0.0200 & 0.0021 \\
\hline$C(1)$ & 0.0921 & 0.0616 & 0.0544 & 0.0384 & 0.0229 & 0.0205 \\
\hline C(39) & 0.0706 & 0.0458 & 0.0756 & 0.0307 & 0.0342 & 0.0130 \\
\hline$C(25)$ & 0.1035 & 0.0686 & 0.0606 & 0.0433 & 0.0360 & 0.0141 \\
\hline$C(35)$ & 0.0793 & 0.0524 & 0.0561 & 0.0328 & 0.0149 & 0.0214 \\
\hline$C(36)$ & 0.0621 & 0.0742 & 0.0608 & 0.0152 & 0.0391 & 0.0162 \\
\hline$C(14)$ & 0.0746 & 0.0786 & 0.0488 & 0.0170 & 0.0341 & 0.0086 \\
\hline$C(19)$ & 0.0759 & 0.0430 & 0.0775 & 0.0023 & 0.0293 & 0.0112 \\
\hline$C(5)$ & 0.0948 & 0.0688 & 0.0630 & 0.0510 & 0.0344 & 0.0259 \\
\hline$C(16)$ & 0.0572 & 0.0934 & 0.0747 & 0.0479 & 0.0259 & 0.0306 \\
\hline$C(15)$ & 0.0665 & 0.0636 & 0.0533 & 0.0207 & 0.0242 & 0.0270 \\
\hline$C(21)$ & 0.1094 & 0.0800 & 0.0599 & 0.0576 & 0.0433 & 0.0271 \\
\hline$C(40)$ & 0.0829 & 0.0732 & 0.0544 & 0.0251 & 0.0244 & 0.0051 \\
\hline C(34) & 0.0685 & 0.0754 & 0.0740 & 0.0456 & 0.0119 & 0.0187 \\
\hline
\end{tabular}


Table 2. Anisotropic Displacement Parameters (continued)

$\begin{array}{lcccccc}\text { atom } & \mathrm{U}_{11} & \mathrm{U}_{22} & \mathrm{U}_{33} & \mathrm{U}_{12} & \mathrm{U}_{13} & \mathrm{U}_{23} \\ \mathrm{C}(20) & 0.0570 & 0.0856 & 0.0614 & 0.0065 & 0.0062 & 0.0068 \\ \mathrm{C}(4) & 0.1418 & 0.1129 & 0.0656 & 0.0773 & 0.0533 & 0.0518 \\ \mathrm{C}(23) & 0.1229 & 0.1367 & 0.0457 & 0.0749 & 0.0195 & 0.0122 \\ \mathrm{C}(2) & 0.1407 & 0.0794 & 0.0566 & 0.0645 & 0.0137 & -0.0001 \\ \mathrm{C}(3) & 0.1590 & 0.1096 & 0.0470 & 0.0870 & 0.0389 & 0.0208 \\ \mathrm{C}(22) & 0.1630 & 0.1241 & 0.0547 & 0.0995 & 0.0430 & 0.0354 \\ \mathrm{C}(24) & 0.1149 & 0.0988 & 0.0635 & 0.0296 & 0.0293 & -0.0144\end{array}$

The general temperature factor expression: $\exp \left(-2 \pi^{2}\left(a^{\star 2} U_{11} h^{2}+b^{*} 2 U_{22} k^{2}+\left.c^{\star 2} U_{33}\right|^{2}\right.\right.$ $\left.\left.+2 a^{*} b^{*} U_{12} h k+2 a^{*} c^{*} U_{13} h l+2 b^{*} c^{*} U_{23} k l\right)\right)$ 
Table 3. Bond lengths $(\AA ̊)$

$\begin{array}{llllll}\text { atom } & \text { atom } & \text { distance } & \text { atom } & \text { atom } & \text { distance } \\ \mathrm{N}(3) & \mathrm{C}(29) & 1.13(1) & \mathrm{N}(4) & \mathrm{C}(30) & 1.13(1) \\ \mathrm{N}(1) & \mathrm{C}(9) & 1.13(1) & \mathrm{N}(2) & \mathrm{C}(10) & 1.14(1) \\ \mathrm{C}(10) & \mathrm{C}(8) & 1.47(1) & \mathrm{C}(8) & \mathrm{C}(11) & 1.58(1) \\ \mathrm{C}(8) & \mathrm{C}(7) & 1.57(1) & \mathrm{C}(8) & \mathrm{C}(9) & 1.48(1) \\ \mathrm{C}(30) & \mathrm{C}(28) & 1.48(1) & \mathrm{C}(29) & \mathrm{C}(28) & 1.48(1) \\ \mathrm{C}(28) & \mathrm{C}(31) & 1.55(1) & \mathrm{C}(28) & \mathrm{C}(27) & 1.58(1) \\ \mathrm{C}(11) & \mathrm{C}(12) & 1.55(2) & \mathrm{C}(11) & \mathrm{C}(17) & 1.572(9) \\ \mathrm{C}(37) & \mathrm{C}(38) & 1.563(9) & \mathrm{C}(37) & \mathrm{C}(31) & 1.58(1) \\ \mathrm{C}(37) & \mathrm{C}(35) & 1.55(1) & \mathrm{C}(37) & \mathrm{C}(36) & 1.50(1) \\ \mathrm{C}(7) & \mathrm{C}(6) & 1.51(1) & \mathrm{C}(26) & \mathrm{C}(27) & 1.51(1) \\ \mathrm{C}(26) & \mathrm{C}(25) & 1.38(1) & \mathrm{C}(26) & \mathrm{C}(21) & 1.37(2) \\ \mathrm{C}(38) & \mathrm{C}(33) & 1.53(2) & \mathrm{C}(38) & \mathrm{C}(39) & 1.52(1) \\ \mathrm{C}(38) & \mathrm{C}(40) & 1.53(1) & \mathrm{C}(6) & \mathrm{C}(1) & 1.39(1) \\ \mathrm{C}(6) & \mathrm{C}(5) & 1.39(1) & \mathrm{C}(31) & \mathrm{C}(32) & 1.55(1) \\ \mathrm{C}(12) & \mathrm{C}(13) & 1.53(1) & \mathrm{C}(32) & \mathrm{C}(33) & 1.53(1) \\ \mathrm{C}(33) & \mathrm{C}(34) & 1.54(2) & \mathrm{C}(18) & \mathrm{C}(17) & 1.57(1) \\ \mathrm{C}(18) & \mathrm{C}(13) & 1.52(2) & \mathrm{C}(18) & \mathrm{C}(19) & 1.52(1) \\ \mathrm{C}(18) & \mathrm{C}(20) & 1.54(1) & \mathrm{C}(17) & \mathrm{C}(16) & 1.51(2) \\ \mathrm{C}(17) & \mathrm{C}(15) & 1.55(1) & \mathrm{C}(13) & \mathrm{C}(14) & 1.54(1) \\ \mathrm{C}(1) & \mathrm{C}(2) & 1.38(2) & \mathrm{C}(25) & \mathrm{C}(24) & 1.39(1) \\ \mathrm{C}(35) & \mathrm{C}(34) & 1.55(1) & \mathrm{C}(14) & \mathrm{C}(15) & 1.54(2) \\ \mathrm{C}(5) & \mathrm{C}(4) & 1.39(2) & \mathrm{C}(21) & \mathrm{C}(22) & 1.39(1) \\ \mathrm{C}(4) & \mathrm{C}(3) & 1.35(2) & \mathrm{C}(23) & \mathrm{C}(22) & 1.34(2) \\ \mathrm{C}(23) & \mathrm{C}(24) & 1.32(2) & \mathrm{C}(2) & \mathrm{C}(3) & 1.36(2) \\ & & & & & \end{array}$


Table 4. Bond lengths involving hydrogens $(\AA)$

$\begin{array}{llllll}\text { atom } & \text { atom } & \text { distance } & \text { atom } & \text { atom } & \text { distance } \\ \mathrm{C}(11) & \mathrm{H}(8) & 0.95(1) & \mathrm{C}(7) & \mathrm{H}(6) & 0.95(2) \\ \mathrm{C}(7) & \mathrm{H}(7) & 0.95(1) & \mathrm{C}(31) & \mathrm{H}(32) & 0.95(1) \\ \mathrm{C}(12) & \mathrm{H}(9) & 0.95(1) & \mathrm{C}(12) & \mathrm{H}(10) & 0.95(1) \\ \mathrm{C}(32) & \mathrm{H}(33) & 0.95(2) & \mathrm{C}(32) & \mathrm{H}(34) & 0.95(1) \\ \mathrm{C}(33) & \mathrm{H}(35) & 0.950(9) & \mathrm{C}(27) & \mathrm{H}(30) & 0.95(1) \\ \mathrm{C}(27) & \mathrm{H}(31) & 0.95(2) & \mathrm{C}(13) & \mathrm{H}(11) & 0.95(2) \\ \mathrm{C}(1) & \mathrm{H}(1) & 0.95(2) & \mathrm{C}(39) & \mathrm{H}(43) & 0.95(1) \\ \mathrm{C}(39) & \mathrm{H}(44) & 0.95(1) & \mathrm{C}(39) & \mathrm{H}(45) & 0.95(2) \\ \mathrm{C}(25) & \mathrm{H}(29) & 0.95(2) & \mathrm{C}(35) & \mathrm{H}(38) & 0.95(2) \\ \mathrm{C}(35) & \mathrm{H}(39) & 0.95(1) & \mathrm{C}(36) & \mathrm{H}(40) & 0.95(2) \\ \mathrm{C}(36) & \mathrm{H}(41) & 0.95(2) & \mathrm{C}(36) & \mathrm{H}(42) & 0.95(1) \\ \mathrm{C}(14) & \mathrm{H}(12) & 0.95(1) & \mathrm{C}(14) & \mathrm{H}(13) & 0.95(1) \\ \mathrm{C}(19) & \mathrm{H}(19) & 0.95(2) & \mathrm{C}(19) & \mathrm{H}(20) & 0.95(2) \\ \mathrm{C}(19) & \mathrm{H}(21) & 0.95(1) & \mathrm{C}(5) & \mathrm{H}(5) & 0.95(1) \\ \mathrm{C}(16) & \mathrm{H}(16) & 0.95(2) & \mathrm{C}(16) & \mathrm{H}(17) & 0.95(1) \\ \mathrm{C}(16) & \mathrm{H}(18) & 0.95(2) & \mathrm{C}(15) & \mathrm{H}(14) & 0.95(1) \\ \mathrm{C}(15) & \mathrm{H}(15) & 0.95(1) & \mathrm{C}(21) & \mathrm{H}(25) & 0.95(1) \\ \mathrm{C}(40) & \mathrm{H}(46) & 0.95(2) & \mathrm{C}(40) & \mathrm{H}(47) & 0.95(1) \\ \mathrm{C}(40) & \mathrm{H}(48) & 0.95(2) & \mathrm{C}(34) & \mathrm{H}(36) & 0.95(2) \\ \mathrm{C}(34) & \mathrm{H}(37) & 0.95(1) & \mathrm{C}(20) & \mathrm{H}(22) & 0.95(2) \\ \mathrm{C}(20) & \mathrm{H}(23) & 0.95(2) & \mathrm{C}(20) & \mathrm{H}(24) & 0.95(2) \\ \mathrm{C}(4) & \mathrm{H}(4) & 0.95(2) & \mathrm{C}(23) & \mathrm{H}(27) & 0.95(2) \\ \mathrm{C}(2) & \mathrm{H}(2) & 0.95(2) & \mathrm{C}(3) & \mathrm{H}(3) & 0.95(2) \\ \mathrm{C}(22) & \mathrm{H}(26) & 0.95(2) & \mathrm{C}(24) & \mathrm{H}(28) & 0.95(2) \\ & & & & & \end{array}$


Table 5. Bond angles (0)

\begin{tabular}{|c|c|c|c|c|c|c|c|}
\hline atom & atom & atom & angle & atom & atom & atom & angle \\
\hline$C(8)$ & $C(10)$ & $\mathrm{N}(2)$ & $178(1)$ & $C(11)$ & $C(8)$ & C(7) & $109.6(8)$ \\
\hline$C(11)$ & $C(8)$ & C(9) & $117.4(5)$ & $C(11)$ & $C(8)$ & $C(10)$ & 107.7(7) \\
\hline$C(7)$ & $C(8)$ & C(9) & $108.4(7)$ & $C(7)$ & $C(8)$ & $C(10)$ & 107.5(6) \\
\hline$C(9)$ & $C(8)$ & $C(10)$ & $105.8(8)$ & C(28) & $C(30)$ & $\mathrm{N}(4)$ & $178(1)$ \\
\hline C(28) & C(29) & $N(3)$ & 176.0(9) & $C(31)$ & C(28) & C(27) & $107.7(7)$ \\
\hline$C(31)$ & C(28) & $C(30)$ & $110.6(7)$ & $C(31)$ & $\mathrm{C}(28)$ & C(29) & $115.7(6)$ \\
\hline C(27) & C(28) & $C(30)$ & $108.8(6)$ & C(27) & $\mathrm{C}(28)$ & C(29) & $107.1(7)$ \\
\hline$C(30)$ & C(28) & C(29) & $106.6(8)$ & C(12) & $C(11)$ & C(17) & $102.8(6)$ \\
\hline$C(12)$ & $C(11)$ & $C(8)$ & $116.4(7)$ & C(17) & $C(11)$ & $C(8)$ & $121.1(7)$ \\
\hline$C(38)$ & C(37) & C(31) & $104.4(6)$ & C(38) & C(37) & C(35) & $99.8(6)$ \\
\hline$C(38)$ & C(37) & $C(36)$ & 116.2(8) & C(31) & $C(37)$ & C(35) & $102.4(8)$ \\
\hline$C(31)$ & C(37) & $C(36)$ & $118.1(6)$ & C(35) & $C(37)$ & C(36) & $113.6(8)$ \\
\hline$C(6)$ & $\mathrm{C}(7)$ & $C(8)$ & 116.1(9) & C(27) & $C(26)$ & C(25) & $121.0(9$ \\
\hline$C(27)$ & $C(26)$ & $C(21)$ & $121.2(7)$ & C(25) & $C(26)$ & $C(21)$ & $117.8(8$ \\
\hline$C(33)$ & $C(38)$ & C(39) & 115.2(8) & C(33) & $C(38)$ & C(40) & $113.1(8$ \\
\hline C(33) & C(38) & $C(37)$ & $94.0(7)$ & C(39) & $C(38)$ & C(40) & $105.2(9$ \\
\hline$C(39)$ & C(38) & $C(37)$ & $116.0(6)$ & $C(40)$ & C(38) & C(37) & $113.5(7)$ \\
\hline$C(1)$ & $C(6)$ & C(5) & $118.8(8)$ & $C(1)$ & C(6) & $C(7)$ & $120.5(8)$ \\
\hline C(5) & $C(6)$ & $C(7)$ & $120.6(7)$ & C(32) & $C(31)$ & C(28) & $115.5(7$ \\
\hline $\mathrm{C}(32)$ & C(31) & C(37) & $102.6(5)$ & C(28) & $C(31)$ & C(37) & $123.0(8$ \\
\hline$N(1)$ & $\mathrm{C}(9)$ & $C(8)$ & $175.8(9)$ & C(13) & C(12) & $C(11)$ & $103.0(8$ \\
\hline C(33) & C(32) & $C(31)$ & $102.9(7)$ & C(34) & C(33) & C(38) & $102.6(8)$ \\
\hline$C(34)$ & $C(33)$ & $C(32)$ & $107.3(7)$ & $C(38)$ & $C(33)$ & C(32) & $102.2(7$ \\
\hline$C(17)$ & $C(18)$ & $C(13)$ & $94.1(6)$ & C(17) & $C(18)$ & C(19) & $115.4(8$ \\
\hline$C(17)$ & $C(18)$ & $C(20)$ & $113.1(8)$ & $C(13)$ & $C(18)$ & C(19) & 114.5 (c \\
\hline$C(13)$ & $C(18)$ & $C(20)$ & $113.8(9)$ & C(19) & $C(18)$ & $C(20)$ & $106.1(7)$ \\
\hline$C(16)$ & $C(17)$ & $C(15)$ & $112.1(9)$ & $C(16)$ & $C(17)$ & $C(11)$ & $118.3(7)$ \\
\hline$C(16)$ & $C(17)$ & $C(18)$ & $117.8(7)$ & $C(15)$ & $C(17)$ & $C(11)$ & $102.9(6$ \\
\hline$C(15)$ & C(17) & $C(18)$ & $99.8(7)$ & $C(11)$ & $C(17)$ & C(18) & $103.5(7$ \\
\hline$C(28)$ & C(27) & $C(26)$ & $115.9(9)$ & $C(14)$ & $C(13)$ & C(12) & $107.7(7$ \\
\hline$C(14)$ & $C(13)$ & C(18) & 103.1(9) & C(12) & C(13) & C(18) & $102.1(8)$ \\
\hline$C(2)$ & C(1) & $C(6)$ & 120(1) & $C(24)$ & $C(25)$ & $C(26)$ & $120(1)$ \\
\hline$C(34)$ & C(35) & C(37) & $103.1(8)$ & C(15) & $C(14)$ & $C(13)$ & $103(1)$ \\
\hline$C(4)$ & C(5) & $C(6)$ & 119.3(9) & C(17) & $C(15)$ & $C(14)$ & $103.7(9$ \\
\hline$C(22)$ & $C(21)$ & $C(26)$ & $120(1)$ & $C(33)$ & C(34) & C(35) & $103.6(9)$ \\
\hline$C(3)$ & $C(4)$ & C(5) & $120(1)$ & C(22) & $C(23)$ & C(24) & $120(1)$ \\
\hline$C(3)$ & $C(2)$ & $C(1)$ & $119(1)$ & C(4) & $C(3)$ & $C(2)$ & $120(1)$ \\
\hline
\end{tabular}


Table 5. Bond angles (0) -- continued

atom atom atom angle atom atom atom angle

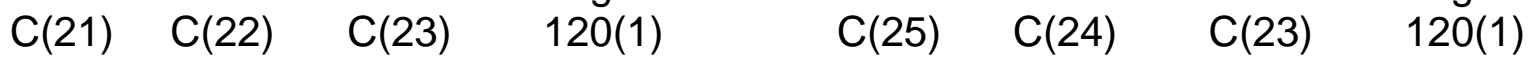


Table 6. Bond angles involving hydrogens ( $\left.{ }^{\circ}\right)$

\begin{tabular}{|c|c|c|c|c|c|c|c|}
\hline atom & atom & atom & angle & atom & atom & atom & angle \\
\hline$C(12)$ & $C(11)$ & $\mathrm{H}(8)$ & 105(1) & $C(17)$ & $C(11)$ & $\mathrm{H}(8)$ & $104.9(8)$ \\
\hline $\mathrm{H}(8)$ & $C(11)$ & $\mathrm{C}(8)$ & $104.8(7)$ & $C(6)$ & $C(7)$ & $\mathrm{H}(6)$ & $107.4(9)$ \\
\hline$C(6)$ & $C(7)^{\prime}$ & $\mathrm{H}(7)$ & $108.0(7)$ & $H(6)$ & $C(7)$ & $\mathrm{H}(7)$ & 109(1) \\
\hline $\mathrm{H}(6)$ & $\mathrm{C}(7)$ & C(8) & $107.7(7)$ & $\mathrm{H}(7)$ & $C(7)$ & $C(8)$ & $108.0(9)$ \\
\hline$C(32)$ & $C(31)$ & $\mathrm{H}(32)$ & 105.2(9) & $H(32)$ & $C(31)$ & C(28) & $104.4(7)$ \\
\hline $\mathrm{H}(32)$ & $C(31)$ & $\mathrm{C}(37)$ & $104.5(9)$ & C(13) & $C(12)$ & $H(9)$ & $110(1)$ \\
\hline$C(13)$ & $C(12)$ & $\mathrm{H}(10)$ & $111.9(8)$ & $H(9)$ & $C(12)$ & $\mathrm{H}(10)$ & $109(1)$ \\
\hline$H(9)$ & $C(12)$ & $C(11)$ & $111(1)$ & $H(10)$ & $C(12)$ & $C(11)$ & $110(1)$ \\
\hline$C(33)$ & $C(32)$ & $\mathrm{H}(33)$ & $111.2(9)$ & C(33) & C(32) & $\mathrm{H}(34)$ & $111.5(9)$ \\
\hline $\mathrm{H}(33)$ & $C(32)$ & $\mathrm{H}(34)$ & $109(1)$ & $\mathrm{H}(33)$ & C(32) & $C(31)$ & 111.4(8) \\
\hline$H(34)$ & $C(32)$ & $C(31)$ & $110.3(9)$ & $C(34)$ & C(33) & $H(35)$ & $114(1)$ \\
\hline$H(35)$ & $C(33)$ & C(38) & $114(1)$ & $\mathrm{H}(35)$ & C(33) & $C(32)$ & 114(1) \\
\hline $\mathrm{H}(30)$ & C(27) & $\mathrm{H}(31)$ & $109(1)$ & $\mathrm{H}(30)$ & $C(27)$ & C(28) & $108(1)$ \\
\hline $\mathrm{H}(30)$ & C(27) & $C(26)$ & $107(1)$ & $\mathrm{H}(31)$ & $C(27)$ & C(28) & $107(1)$ \\
\hline $\mathrm{H}(31)$ & C(27) & $C(26)$ & $107.6(9)$ & C(14) & $C(13)$ & $\mathrm{H}(11)$ & $114(1)$ \\
\hline$H(11)$ & $C(13)$ & $\mathrm{C}(12)$ & $114(1)$ & $\mathrm{H}(11)$ & $C(13)$ & C(18) & $114.1(9)$ \\
\hline$C(2)$ & $C(1)$ & $\mathrm{H}(1)$ & $121(1)$ & $H(1)$ & $C(1)$ & $C(6)$ & $118(1)$ \\
\hline $\mathrm{H}(43)$ & C(39) & $\mathrm{H}(44)$ & $109(1)$ & $\mathrm{H}(43)$ & C(39) & $\mathrm{H}(45)$ & $109(1)$ \\
\hline $\mathrm{H}(43)$ & C(39) & $\mathrm{C}(38)$ & $110(1)$ & $\mathrm{H}(44)$ & C(39) & $\mathrm{H}(45)$ & $109(1)$ \\
\hline $\mathrm{H}(44)$ & $C(39)$ & C(38) & $108(1)$ & $\mathrm{H}(45)$ & C(39) & $C(38)$ & 109(1) \\
\hline$C(24)$ & $C(25)$ & $\mathrm{H}(29)$ & $120(1)$ & $\mathrm{H}(29)$ & C(25) & $C(26)$ & 118(1) \\
\hline$C(34)$ & C(35) & $\mathrm{H}(38)$ & $111.0(9)$ & C(34) & $C(35)$ & $\mathrm{H}(39)$ & 111(1) \\
\hline $\mathrm{H}(38)$ & C(35) & $\mathrm{H}(39)$ & 109(1) & $\mathrm{H}(38)$ & $C(35)$ & C(37) & 111(1) \\
\hline $\mathrm{H}(39)$ & C(35) & $\mathrm{C}(37)$ & $110.3(9)$ & $\mathrm{H}(40)$ & $C(36)$ & $\mathrm{H}(41)$ & $109(1)$ \\
\hline $\mathrm{H}(40)$ & $C(36)$ & $\mathrm{H}(42)$ & 109(1) & $\mathrm{H}(40)$ & $C(36)$ & C(37) & $110.0(9)$ \\
\hline $\mathrm{H}(41)$ & $C(36)$ & $\mathrm{H}(42)$ & $109(1)$ & $\mathrm{H}(41)$ & $C(36)$ & C(37) & $109(1)$ \\
\hline $\mathrm{H}(42)$ & $C(36)$ & C(37) & $109(1)$ & $C(15)$ & $C(14)$ & $\mathrm{H}(12)$ & 111(1) \\
\hline$C(15)$ & $C(14)$ & $\mathrm{H}(13)$ & 111(1) & $H(12)$ & $C(14)$ & $H(13)$ & $109(1)$ \\
\hline$H(12)$ & $C(14)$ & $C(13)$ & 109(1) & $\mathrm{H}(13)$ & $C(14)$ & $C(13)$ & $112(1)$ \\
\hline$H(19)$ & $C(19)$ & $\mathrm{H}(20)$ & $109(1)$ & $\mathrm{H}(19)$ & $C(19)$ & $\mathrm{H}(21)$ & $109(1)$ \\
\hline $\mathrm{H}(19)$ & C(19) & $C(18)$ & $110(1)$ & $\mathrm{H}(20)$ & $C(19)$ & $\mathrm{H}(21)$ & $109(1)$ \\
\hline $\mathrm{H}(20)$ & C(19) & $C(18)$ & $109(1)$ & $\mathrm{H}(21)$ & $C(19)$ & C(18) & $108(1)$ \\
\hline$C(4)$ & $C(5)$ & $\mathrm{H}(5)$ & 121(1) & $H(5)^{\prime}$ & $C(5)^{\prime}$ & $C(6)$ & $118(1)$ \\
\hline $\mathrm{H}(16)$ & $C(16)$ & $\mathrm{H}(17)$ & 109(1) & $H(16)$ & $C(16)$ & $H(18)$ & 109(1) \\
\hline$H(16)$ & $C(16)$ & $\mathrm{C}(17)$ & $109(1)$ & $\mathrm{H}(17)$ & $C(16)$ & $H(18)$ & $109(1)$ \\
\hline $\mathrm{H}(17)$ & $C(16)$ & $\mathrm{C}(17)$ & $110(1)$ & $\mathrm{H}(18)$ & $C(16)$ & $C(17)$ & 108(1) \\
\hline $\mathrm{H}(14)$ & $C(15)$ & $\mathrm{H}(15)$ & $109(1)$ & $\mathrm{H}(14)$ & $C(15)$ & $C(17)$ & 111(1) \\
\hline
\end{tabular}


Table 6. Bond angles involving hydrogens $\left({ }^{0}\right)$-- continued

$\begin{array}{llllllll}\text { atom } & \text { atom } & \text { atom } & \text { angle } & \text { atom } & \text { atom } & \text { atom } & \text { angle } \\ \mathrm{H}(14) & \mathrm{C}(15) & \mathrm{C}(14) & 110(1) & \mathrm{H}(15) & \mathrm{C}(15) & \mathrm{C}(17) & 109(1) \\ \mathrm{H}(15) & \mathrm{C}(15) & \mathrm{C}(14) & 110(1) & \mathrm{C}(22) & \mathrm{C}(21) & \mathrm{H}(25) & 120(1) \\ \mathrm{H}(25) & \mathrm{C}(21) & \mathrm{C}(26) & 119(1) & \mathrm{H}(46) & \mathrm{C}(40) & \mathrm{H}(47) & 109(1) \\ \mathrm{H}(46) & \mathrm{C}(40) & \mathrm{H}(48) & 109(1) & \mathrm{H}(46) & \mathrm{C}(40) & \mathrm{C}(38) & 109(1) \\ \mathrm{H}(47) & \mathrm{C}(40) & \mathrm{H}(48) & 109(1) & \mathrm{H}(47) & \mathrm{C}(40) & \mathrm{C}(38) & 109(1) \\ \mathrm{H}(48) & \mathrm{C}(40) & \mathrm{C}(38) & 110(1) & \mathrm{H}(36) & \mathrm{C}(34) & \mathrm{H}(37) & 109(1) \\ \mathrm{H}(36) & \mathrm{C}(34) & \mathrm{C}(33) & 110(1) & \mathrm{H}(36) & \mathrm{C}(34) & \mathrm{C}(35) & 110.6(9) \\ \mathrm{H}(37) & \mathrm{C}(34) & \mathrm{C}(33) & 111(1) & \mathrm{H}(37) & \mathrm{C}(34) & \mathrm{C}(35) & 111(1) \\ \mathrm{H}(22) & \mathrm{C}(20) & \mathrm{H}(23) & 109(1) & \mathrm{H}(22) & \mathrm{C}(20) & \mathrm{H}(24) & 109(1) \\ \mathrm{H}(22) & \mathrm{C}(20) & \mathrm{C}(18) & 110(1) & \mathrm{H}(23) & \mathrm{C}(20) & \mathrm{H}(24) & 109(1) \\ \mathrm{H}(23) & \mathrm{C}(20) & \mathrm{C}(18) & 109.5(9) & \mathrm{H}(24) & \mathrm{C}(20) & \mathrm{C}(18) & 108(1) \\ \mathrm{C}(3) & \mathrm{C}(4) & \mathrm{H}(4) & 119(1) & \mathrm{H}(4) & \mathrm{C}(4) & \mathrm{C}(5) & 119(1) \\ \mathrm{C}(22) & \mathrm{C}(23) & \mathrm{H}(27) & 118(2) & \mathrm{C}(24) & \mathrm{C}(23) & \mathrm{H}(27) & 120(1) \\ \mathrm{C}(3) & \mathrm{C}(2) & \mathrm{H}(2) & 120(1) & \mathrm{H}(2) & \mathrm{C}(2) & \mathrm{C}(1) & 119(1) \\ \mathrm{H}(3) & \mathrm{C}(3) & \mathrm{C}(4) & 119(1) & \mathrm{H}(3) & \mathrm{C}(3) & \mathrm{C}(2) & 120(1) \\ \mathrm{H}(26) & \mathrm{C}(22) & \mathrm{C}(21) & 119(1) & \mathrm{H}(26) & \mathrm{C}(22) & \mathrm{C}(23) & 119(1) \\ \mathrm{H}(28) & \mathrm{C}(24) & \mathrm{C}(25) & 121(1) & \mathrm{H}(28) & \mathrm{C}(24) & \mathrm{C}(23) & 118(1)\end{array}$


Table 7. Torsion Angles( $\left({ }^{\circ}\right)$

atom1 atom2 atom3 atom4 angle

$\mathrm{C}(10) \quad \mathrm{C}(8) \quad \mathrm{C}(11) \quad \mathrm{C}(12) \quad 159.7(6)$

$\begin{array}{lllll}\mathrm{C}(10) & \mathrm{C}(8) & \mathrm{C}(11) & \mathrm{H}(8) & 43(1)\end{array}$

$\begin{array}{lllll}\mathrm{C}(7) & \mathrm{C}(8) & \mathrm{C}(11) & \mathrm{C}(17) & 169.2(7)\end{array}$

$\begin{array}{lllll}\mathrm{C}(9) & \mathrm{C}(8) & \mathrm{C}(11) & \mathrm{C}(12) & -81.1(9)\end{array}$

$\begin{array}{lllll}\mathrm{C}(9) & \mathrm{C}(8) & \mathrm{C}(11) & \mathrm{H}(8) & 163(1)\end{array}$

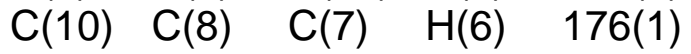

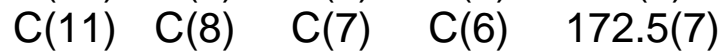

$\begin{array}{lllll}\mathrm{C}(11) & \mathrm{C}(8) & \mathrm{C}(7) & \mathrm{H}(7) & 51(1)\end{array}$

$\begin{array}{lllll}\mathrm{C}(9) & \mathrm{C}(8) & \mathrm{C}(7) & \mathrm{H}(6) & 62(1)\end{array}$

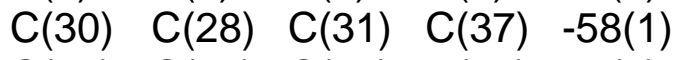

$\mathrm{C}(30) \quad \mathrm{C}(28) \mathrm{C}(31) \mathrm{H}(32) \quad 59(1)$

C(29) C(28) C(31) C(32) $-63(1)$

C(27) $\quad \mathrm{C}(28) \quad \mathrm{C}(31) \quad \mathrm{C}(37)-177.2(6)$

$\begin{array}{lllll}\mathrm{C}(27) & \mathrm{C}(28) & \mathrm{C}(31) & \mathrm{H}(32) & -58(1)\end{array}$

$\begin{array}{lllll}\mathrm{C}(30) & \mathrm{C}(28) & \mathrm{C}(27) & \mathrm{H}(30) & 170(1)\end{array}$

$\begin{array}{lllll}\mathrm{C}(29) & \mathrm{C}(28) & \mathrm{C}(27) & \mathrm{C}(26) & -65.4(8)\end{array}$

$\begin{array}{lllll}\mathrm{C}(29) & \mathrm{C}(28) & \mathrm{C}(27) & \mathrm{H}(31) & 174.2(7)\end{array}$

$\begin{array}{lllll}\mathrm{C}(31) & \mathrm{C}(28) & \mathrm{C}(27) & \mathrm{H}(30) & -69(1)\end{array}$

C(8) C(11) C(12) C(13) 145.3(6)

C(8) $\quad \mathrm{C}(11) \quad \mathrm{C}(12) \quad \mathrm{H}(10) \quad 25.5(9)$

$\begin{array}{lllll}\mathrm{C}(17) & \mathrm{C}(11) & \mathrm{C}(12) & \mathrm{H}(9) & 128.7(9)\end{array}$

$\mathrm{H}(8) \quad \mathrm{C}(11) \quad \mathrm{C}(12) \quad \mathrm{C}(13) \quad-99.1(8)$

$\mathrm{H}(8) \quad \mathrm{C}(11) \quad \mathrm{C}(12) \quad \mathrm{H}(10) \quad 141.1(8)$

$\mathrm{C}(8) \quad \mathrm{C}(11) \quad \mathrm{C}(17) \quad \mathrm{C}(16) \quad 25(1)$

C(12) C(11) C(17) C(18) 24.7(8)

$\begin{array}{lllll}C(12) & C(11) & C(17) & C(15) & -78.8(8)\end{array}$

$\mathrm{H}(8) \quad \mathrm{C}(11) \quad \mathrm{C}(17) \quad \mathrm{C}(16)-93(1)$

$\begin{array}{lllll}\mathrm{C}(31) & \mathrm{C}(37) & \mathrm{C}(38) & \mathrm{C}(33) & -48.3(8)\end{array}$

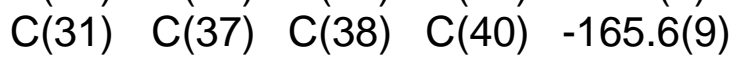

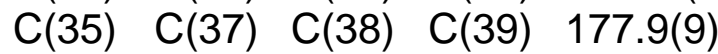

C(36) C(37) C(38) C(33) 179(623)

$\mathrm{C}(36) \quad \mathrm{C}(37) \quad \mathrm{C}(38) \quad \mathrm{C}(40) \quad 62(1)$

$\begin{array}{lllll}\mathrm{C}(38) & \mathrm{C}(37) & \mathrm{C}(31) & \mathrm{C}(32) & 23.0(9)\end{array}$

C(35) $\quad \mathrm{C}(37) \quad \mathrm{C}(31) \quad \mathrm{C}(28) \quad 147.2(6)$

$\begin{array}{lllll}\mathrm{C}(35) & \mathrm{C}(37) & \mathrm{C}(31) & \mathrm{H}(32) & 28.9(7)\end{array}$

$\begin{array}{lllll}\mathrm{C}(36) & \mathrm{C}(37) & \mathrm{C}(31) & \mathrm{C}(32) & 153.8(7)\end{array}$

$\begin{array}{lllll}C(38) & C(37) & C(35) & C(34) & -39(1)\end{array}$ atom1 atom2 atom3 atom4 angle

$\begin{array}{lllll}C(10) & C(8) & C(11) & C(17) & -74.2(8)\end{array}$

$\begin{array}{lllll}\mathrm{C}(7) & \mathrm{C}(8) & \mathrm{C}(11) & \mathrm{C}(12) & 43.1(8)\end{array}$

$\begin{array}{lllll}\mathrm{C}(7) & \mathrm{C}(8) & \mathrm{C}(11) & \mathrm{H}(8) & -72(1)\end{array}$

$\begin{array}{lllll}\mathrm{C}(9) & \mathrm{C}(8) & \mathrm{C}(11) & \mathrm{C}(17) & 44(1)\end{array}$

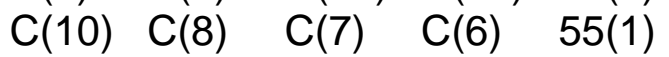

$\begin{array}{lllll}\mathrm{C}(10) & \mathrm{C}(8) & \mathrm{C}(7) & \mathrm{H}(7) & -65(1)\end{array}$

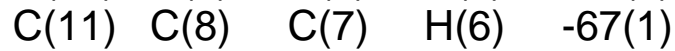

$\begin{array}{lllll}\mathrm{C}(9) & \mathrm{C}(8) & \mathrm{C}(7) & \mathrm{C}(6) & -58(1)\end{array}$

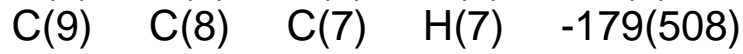

C(30) C(28) C(31) C(32) 174.9(8)

C(29) $\quad$ (28) C(31) C(37) $63.0(9)$

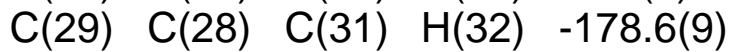

$\mathrm{C}(27) \quad \mathrm{C}(28) \quad \mathrm{C}(31) \quad \mathrm{C}(32) \quad 56.1(9)$

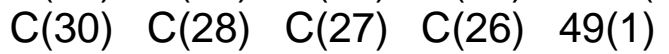

$\begin{array}{lllll}\mathrm{C}(30) & \mathrm{C}(28) & \mathrm{C}(27) & \mathrm{H}(31) & -70.9(9)\end{array}$

$\mathrm{C}(29) \quad \mathrm{C}(28) \quad \mathrm{C}(27) \quad \mathrm{H}(30) \quad 55(1)$

$\begin{array}{lllll}\mathrm{C}(31) & \mathrm{C}(28) & \mathrm{C}(27) & \mathrm{C}(26) & 169.5(7)\end{array}$

$\begin{array}{lllll}\mathrm{C}(31) & \mathrm{C}(28) & \mathrm{C}(27) & \mathrm{H}(31) & 49.1(8)\end{array}$

$\begin{array}{lllll}\mathrm{C}(8) \quad \mathrm{C}(11) & \mathrm{C}(12) & \mathrm{H}(9) & -96(1)\end{array}$

C(17) C(11) C(12) C(13) 10.5(8)

$\begin{array}{lllll}\mathrm{C}(17) & \mathrm{C}(11) & \mathrm{C}(12) & \mathrm{H}(10) & -109.3(8)\end{array}$

$\begin{array}{lllll}\mathrm{H}(8) & \mathrm{C}(11) & \mathrm{C}(12) & \mathrm{H}(9) & 19(1)\end{array}$

$\begin{array}{lllll}C(8) & C(11) & C(17) & C(18) & -107.3(9)\end{array}$

$\begin{array}{lllll}\mathrm{C}(8) & \mathrm{C}(11) & \mathrm{C}(17) & \mathrm{C}(15) & 149.2(8)\end{array}$

C(12) C(11) C(17) C(16) 157.1(8)

$\mathrm{H}(8) \quad \mathrm{C}(11) \quad \mathrm{C}(17) \quad \mathrm{C}(18) \quad 134(1)$

$\mathrm{H}(8) \quad \mathrm{C}(11) \quad \mathrm{C}(17) \quad \mathrm{C}(15) \quad 31(1)$

C(31) C(37) C(38) C(39) 72(1)

$\begin{array}{lllll}C(35) & C(37) & C(38) & C(33) & 57.3(8)\end{array}$

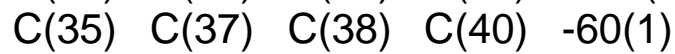

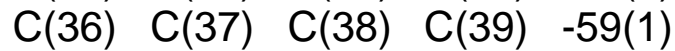

$\begin{array}{lllll}C(38) & C(37) & C(31) & C(28) & -109.2(7)\end{array}$

$\begin{array}{lllll}\mathrm{C}(38) & \mathrm{C}(37) & \mathrm{C}(31) & \mathrm{H}(32) & 132.5(8)\end{array}$

$\begin{array}{lllll}\mathrm{C}(35) & \mathrm{C}(37) & \mathrm{C}(31) & \mathrm{C}(32) & -80.7(7)\end{array}$

C(36) C(37) C(31) C(28) 21(1)

$\begin{array}{lllll}\mathrm{C}(36) & \mathrm{C}(37) & \mathrm{C}(31) & \mathrm{H}(32) & -96.6(9)\end{array}$

$\begin{array}{lllll}\mathrm{C}(38) & \mathrm{C}(37) & \mathrm{C}(35) & \mathrm{H}(38) & 79(1)\end{array}$ 
Table 7. Torsion angles (0) -- continued

atom1 atom2 atom3 atom4 angle

C(38) $\mathrm{C}(37) \quad \mathrm{C}(35) \quad \mathrm{H}(39) \quad-158(1)$

$\begin{array}{lllll}\mathrm{C}(31) & \mathrm{C}(37) & \mathrm{C}(35) & \mathrm{H}(38) & -173.3(7)\end{array}$

$\begin{array}{lllll}C(36) & C(37) & C(35) & C(34) & -164.0(7)\end{array}$

$\begin{array}{lllll}\mathrm{C}(36) & \mathrm{C}(37) & \mathrm{C}(35) & \mathrm{H}(39) & 76(1)\end{array}$

C(38) $\mathrm{C}(37) \quad \mathrm{C}(36) \mathrm{H}(41) \quad 64(1)$

$\mathrm{C}(31) \quad \mathrm{C}(37) \mathrm{C}(36) \mathrm{H}(40) \quad 179(1)$

$\mathrm{C}(31) \quad \mathrm{C}(37) \mathrm{C}(36) \mathrm{H}(42) \quad 59(1)$

$\begin{array}{lllll}\mathrm{C}(35) & \mathrm{C}(37) & \mathrm{C}(36) & \mathrm{H}(41) & 179.5(9)\end{array}$

$\begin{array}{lllll}\mathrm{C}(8) & \mathrm{C}(7) & \mathrm{C}(6) & \mathrm{C}(1) & -97(1)\end{array}$

$\begin{array}{lllll}\mathrm{H}(6) & \mathrm{C}(7) & \mathrm{C}(6) & \mathrm{C}(1) & 142(1)\end{array}$

$\begin{array}{lllll}\mathrm{H}(7) & \mathrm{C}(7) & \mathrm{C}(6) & \mathrm{C}(1) & 24(1)\end{array}$

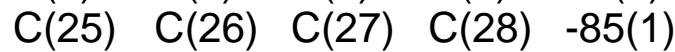

$\begin{array}{lllll}\mathrm{C}(25) & \mathrm{C}(26) & \mathrm{C}(27) & \mathrm{H}(31) & 34(1)\end{array}$

$\begin{array}{lllll}\mathrm{C}(21) & \mathrm{C}(26) & \mathrm{C}(27) & \mathrm{H}(30) & -26(1)\end{array}$

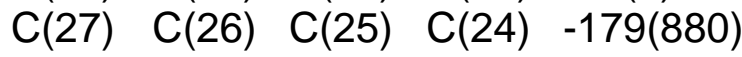

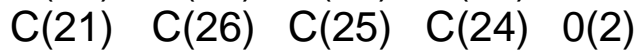

C(27) $\mathrm{C}(26) \quad \mathrm{C}(21) \quad \mathrm{C}(22) \quad 178(1)$

$\begin{array}{lllll}\mathrm{C}(25) & \mathrm{C}(26) & \mathrm{C}(21) & \mathrm{C}(22) & 0(2)\end{array}$

C(37) C(38) C(33) C(32) 56.8(7)

$\begin{array}{lllll}\mathrm{C}(37) & \mathrm{C}(38) & \mathrm{C}(33) & \mathrm{H}(35) & -178(1)\end{array}$

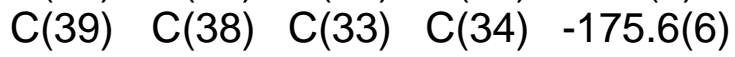

$\mathrm{C}(40) \quad \mathrm{C}(38) \quad \mathrm{C}(33) \quad \mathrm{C}(32) \quad 174.4(6)$

$\begin{array}{lllll}\mathrm{C}(40) & \mathrm{C}(38) & \mathrm{C}(33) & \mathrm{H}(35) & -61(1)\end{array}$

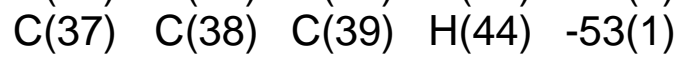

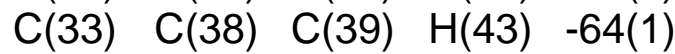

C(33) $\mathrm{C}(38) \quad \mathrm{C}(39) \quad \mathrm{H}(45) \quad 174.8(8)$

$\begin{array}{lllll}\mathrm{C}(40) & \mathrm{C}(38) & \mathrm{C}(39) & \mathrm{H}(44) & -179(359)\end{array}$

$\mathrm{C}(37) \quad \mathrm{C}(38) \mathrm{C}(40) \mathrm{H}(46) \quad 52(1)$

$\mathrm{C}(37) \quad \mathrm{C}(38) \mathrm{C}(40) \quad \mathrm{H}(48) \quad-68(1)$

$\begin{array}{lllll}\mathrm{C}(33) & \mathrm{C}(38) & \mathrm{C}(40) & \mathrm{H}(47) & 66(1)\end{array}$

$\begin{array}{lllll}\mathrm{C}(39) & \mathrm{C}(38) & \mathrm{C}(40) & \mathrm{H}(46) & 179(719)\end{array}$

$\begin{array}{lllll}\mathrm{C}(39) & \mathrm{C}(38) & \mathrm{C}(40) & \mathrm{H}(48) & 59(1)\end{array}$

$\begin{array}{lllll}\mathrm{C}(7) & \mathrm{C}(6) & \mathrm{C}(1) & \mathrm{H}(1) & 3(2)\end{array}$

$\begin{array}{lllll}\mathrm{C}(5) & \mathrm{C}(6) & \mathrm{C}(1) & \mathrm{H}(1) & 179(719)\end{array}$

$\begin{array}{lllll}\mathrm{C}(7) & \mathrm{C}(6) & \mathrm{C}(5) & \mathrm{H}(5) & 0(2)\end{array}$

$\mathrm{C}(1) \quad \mathrm{C}(6) \quad \mathrm{C}(5) \quad \mathrm{H}(5) \quad-176(1)$

$\mathrm{C}(28) \quad \mathrm{C}(31) \quad \mathrm{C}(32) \quad \mathrm{H}(33) \quad-91(1)$ atom1 atom2 atom3 atom4 angle

C(31) C(37) C(35) C(34) 67.6(8)

$\begin{array}{lllll}\mathrm{C}(31) & \mathrm{C}(37) & \mathrm{C}(35) & \mathrm{H}(39) & -51(1)\end{array}$

$\begin{array}{lllll}\mathrm{C}(36) & \mathrm{C}(37) & \mathrm{C}(35) & \mathrm{H}(38) & -44(1)\end{array}$

$\begin{array}{lllll}\mathrm{C}(38) & \mathrm{C}(37) & \mathrm{C}(36) & \mathrm{H}(40) & -55(1)\end{array}$

$\begin{array}{lllll}\mathrm{C}(38) & \mathrm{C}(37) & \mathrm{C}(36) & \mathrm{H}(42) & -175(1)\end{array}$

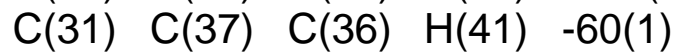

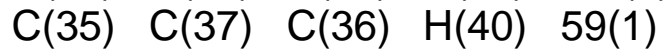

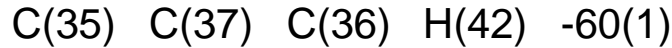

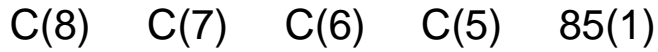

$\begin{array}{lllll}\mathrm{H}(6) & \mathrm{C}(7) & \mathrm{C}(6) & \mathrm{C}(5) & -34(1)\end{array}$

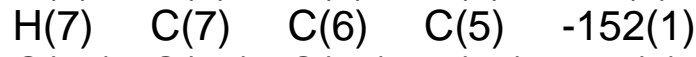

$\begin{array}{lllll}\mathrm{C}(25) & \mathrm{C}(26) & \mathrm{C}(27) & \mathrm{H}(30) & 152(1)\end{array}$

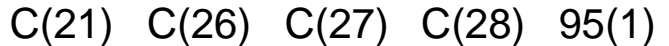

$\begin{array}{lllll}\mathrm{C}(21) & \mathrm{C}(26) & \mathrm{C}(27) & \mathrm{H}(31) & -144(1)\end{array}$

$\begin{array}{lllll}\mathrm{C}(27) & \mathrm{C}(26) & \mathrm{C}(25) & \mathrm{H}(29) & -1(2)\end{array}$

$\begin{array}{lllll}\mathrm{C}(21) & \mathrm{C}(26) & \mathrm{C}(25) & \mathrm{H}(29) & 177(1)\end{array}$

$\begin{array}{lllll}\mathrm{C}(27) & \mathrm{C}(26) & \mathrm{C}(21) & \mathrm{H}(25) & -1(2)\end{array}$

$\begin{array}{lllll}\mathrm{C}(25) & \mathrm{C}(26) & \mathrm{C}(21) & \mathrm{H}(25) & 178(1)\end{array}$

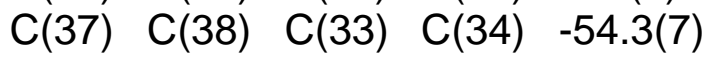

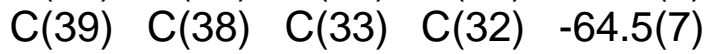

$\begin{array}{lllll}\mathrm{C}(39) & \mathrm{C}(38) & \mathrm{C}(33) & \mathrm{H}(35) & 60(1)\end{array}$

C(40) $\quad \mathrm{C}(38) \quad \mathrm{C}(33) \quad \mathrm{C}(34) \quad 63.3(7)$

$\begin{array}{lllll}\mathrm{C}(37) & \mathrm{C}(38) & \mathrm{C}(39) & \mathrm{H}(43) & -173(1)\end{array}$

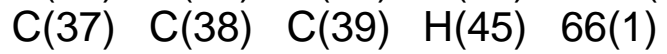

$\mathrm{C}(33) \quad \mathrm{C}(38) \quad \mathrm{C}(39) \mathrm{H}(44) \quad 55(1)$

$\mathrm{C}(40) \quad \mathrm{C}(38) \quad \mathrm{C}(39) \quad \mathrm{H}(43) \quad 60(1)$

$\begin{array}{lllll}\mathrm{C}(40) & \mathrm{C}(38) & \mathrm{C}(39) & \mathrm{H}(45) & -60.0(9)\end{array}$

$\begin{array}{lllll}\mathrm{C}(37) & \mathrm{C}(38) & \mathrm{C}(40) & \mathrm{H}(47) & 171(1)\end{array}$

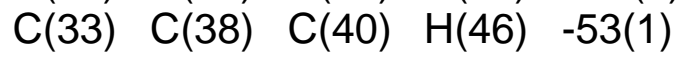

$\begin{array}{lllll}\mathrm{C}(33) & \mathrm{C}(38) & \mathrm{C}(40) & \mathrm{H}(48) & -173(1)\end{array}$

$\begin{array}{lllll}\mathrm{C}(39) & \mathrm{C}(38) & \mathrm{C}(40) & \mathrm{H}(47) & -60(1)\end{array}$

$\begin{array}{lllll}\mathrm{C}(7) & \mathrm{C}(6) & \mathrm{C}(1) & \mathrm{C}(2) & -177(1)\end{array}$

$\begin{array}{lllll}C(5) & C(6) & C(1) & C(2) & -1(2)\end{array}$

$\begin{array}{lllll}\mathrm{C}(7) & \mathrm{C}(6) & \mathrm{C}(5) & \mathrm{C}(4) & 177(1)\end{array}$

$\begin{array}{lllll}C(1) & C(6) & C(5) & C(4) & 0(2)\end{array}$

$\mathrm{C}(28) \quad \mathrm{C}(31) \quad \mathrm{C}(32) \quad \mathrm{C}(33) \quad 148.9(8)$

$\begin{array}{lllll}\mathrm{C}(28) & \mathrm{C}(31) & \mathrm{C}(32) & \mathrm{H}(34) & 29(1)\end{array}$ 
Table 7. Torsion angles (0) -- continued

\begin{tabular}{|c|c|c|c|c|c|c|c|c|c|}
\hline (37) & $C(31)$ & C(32) & C(33) & $12.4(9)$ & $\mathrm{C}(37)$ & $C(31)$ & $C(32)$ & $\mathrm{H}(33)$ & 131(1) \\
\hline$C(37)$ & $C(31)$ & $C(32)$ & $\mathrm{H}(34)$ & $-106(1)$ & $\mathrm{H}(32)$ & $C(31)$ & $C(32)$ & $C(33)$ & $-96(1)$ \\
\hline (32) & $C(31)$ & $C(32)$ & $H(33)$ & $22(1)$ & (32) & $C(31)$ & $C(32)$ & $H(34)$ & 144(1) \\
\hline$C(11)$ & $C(12)$ & $C(13)$ & $C(18)$ & $-43.8(9)$ & $C(11)$ & $C(12)$ & $C(13)$ & $C(14)$ & $64(1)$ \\
\hline (11) & $C(12)$ & (13) & $H(11)$ & & H(9) & $C(12)$ & & $C(18)$ & $-162(1)$ \\
\hline 9) & $C(12)$ & (13) & $C(14)$ & $-54(1)$ & & $C(12)$ & & & $73(1)$ \\
\hline (10) & $C(12)$ & $C(13)$ & $C(18)$ & $75(1)$ & $H(10)$ & $C(12)$ & $C(13)$ & $C(14)$ & $-176(1)$ \\
\hline (10) & $C(12)$ & C(13) & $H(11)$ & $-48(1)$ & C(31) & C(32) & C(33) & C(38) & $-44.8(9)$ \\
\hline (31) & $C(32)$ & C(33) & C(34) & $62(1)$ & $C(31)$ & $C(32)$ & $C(33)$ & $H(35)$ & $-168(1)$ \\
\hline (33) & $C(32)$ & $C(33)$ & $C(38)$ & $-164.1(9)$ & (33) & $C(32)$ & $C(33)$ & $C(34)$ & $-56(1)$ \\
\hline (33) & C(32) & C(33) & $H(35)$ & $71(1)$ & (34) & $C(32)$ & $C(33)$ & C(38) & $73(1)$ \\
\hline (34) & C(32) & C(33) & C(34) & $-179(1)$ & H(34) & $C(32)$ & $C(33)$ & $H(35)$ & $-50(1)$ \\
\hline (38) & $C(33)$ & $C(34)$ & $C(35)$ & $31.2(9)$ & $C(38)$ & $C(33)$ & $C(34)$ & $H(36)$ & 149.5(9) \\
\hline$C(38)$ & $C(33)$ & $C(34)$ & $\mathrm{H}(37)$ & $-88(1)$ & $C(32)$ & $C(33)$ & $C(34)$ & C(35) & $-76(1)$ \\
\hline (32) & $C(33)$ & $C(34)$ & $H(36)$ & $42(1)$ & $C(32)$ & $C(33)$ & $C(34)$ & $\mathrm{H}(37)$ & 164(1) \\
\hline (35) & C(33) & C(34) & C(35) & $155(1)$ & $H(35)$ & C(33) & C(34) & $H(36)$ & $-86(1)$ \\
\hline (35) & C(33) & $C(34)$ & $\mathrm{H}(37)$ & $35(2)$ & $C(13)$ & $C(18)$ & $C(17)$ & $C(11)$ & $-49.7(8)$ \\
\hline$(13$ & $C(18)$ & $C(17)$ & $C(16)$ & $177.7(8)$ & $C(13)$ & $C(18)$ & $C(17)$ & $C(15)$ & $56.3(7)$ \\
\hline (19) & $C(18)$ & $C(17)$ & $C(11)$ & 69.9 & $C(19)$ & $C(18)$ & $C(17)$ & $C(16)$ & $-62(1)$ \\
\hline$C(19)$ & C(18) & $C(17)$ & $C(15)$ & $175.8(7)$ & $\mathrm{C}(20)$ & $C(18)$ & $\mathrm{C}(17)$ & $C(11)$ & $-167.7(\varepsilon$ \\
\hline$C(20)$ & C(18) & $C(17)$ & $C(16)$ & $59(1)$ & $\mathrm{C}(20)$ & $C(18)$ & $\mathrm{C}(17)$ & $C(15)$ & $-61(1)$ \\
\hline$C(17)$ & $C(18)$ & $C(13)$ & $C(12)$ & $57.0(8)$ & $C(17)$ & $C(18)$ & $C(13)$ & $C(14)$ & $-54.6(7)$ \\
\hline & $C(18)$ & $C(13)$ & $H(11)$ & & $C(19)$ & $C(18)$ & $C(13)$ & $C(12)$ & $-63.3(9)$ \\
\hline$C(19)$ & $C(18)$ & $C(13)$ & $C(14)$ & $-174.9(6)$ & $C(19)$ & $C(18)$ & $C(13)$ & $H(11)$ & $60(1)$ \\
\hline$C(20)$ & $C(18)$ & $C(13)$ & $C(12)$ & $174.5(8)$ & $C(20)$ & $C(18)$ & $C(13)$ & $C(14)$ & $62.8(9)$ \\
\hline$C(20)$ & $C(18)$ & $C(13)$ & $H(11)$ & $-61(1)$ & $C(17)$ & $C(18)$ & $C(19)$ & $H(19)$ & $66(1)$ \\
\hline$C(17)$ & $C(18)$ & $C(19)$ & $\mathrm{H}(20)$ & $-172(1)$ & $C(17)$ & $C(18)$ & $C(19)$ & $\mathrm{H}(21)$ & $-52(1)$ \\
\hline C(13) & $C(18)$ & $C(19)$ & $\mathrm{H}(19)$ & $174.5(9)$ & $C(13)$ & $C(18)$ & $C(19)$ & $\mathrm{H}(20)$ & $-65(1)$ \\
\hline$C(13)$ & C(18) & C(19) & $\mathrm{H}(21)$ & $54(1)$ & C(20) & C(18) & C(19) & $H(19)$ & $-59(1)$ \\
\hline$C(20)$ & C(18) & C(19) & $\mathrm{H}(20)$ & $61(1)$ & C(20) & C(18) & C(19) & $H(21)$ & $-179(1)$ \\
\hline$C(17)$ & $C(18)$ & $C(20)$ & $\mathrm{H}(22)$ & $-67(1)$ & $C(17)$ & $C(18)$ & $C(20)$ & $H(23)$ & $52(1)$ \\
\hline$C(17)$ & $C(18)$ & $C(20)$ & $\mathrm{H}(24)$ & $172(1)$ & $C(13)$ & $C(18)$ & $C(20)$ & $\mathrm{H}(22)$ & $-173(1)$ \\
\hline$C(13)$ & $C(18)$ & $C(20)$ & $\mathrm{H}(23)$ & $-53(1)$ & $C(13)$ & $C(18)$ & $C(20)$ & $\mathrm{H}(24)$ & 66(1) \\
\hline$C(19)$ & $C(18)$ & C(20) & $\mathrm{H}(22)$ & $59(1)$ & C(19) & C(18) & $C(20)$ & $\mathrm{H}(23)$ & $179(88$ \\
\hline$C(19)$ & $C(18)$ & $C(20)$ & $\mathrm{H}(24)$ & $-60(1)$ & $C(11)$ & $C(17)$ & $C(16)$ & $H(16)$ & $57(1)$ \\
\hline (11 & $\mathrm{C}(17)$ & $C(16)$ & $\mathrm{H}(17)$ & 178(1) & $C(11)$ & $C(17)$ & $C(16)$ & $H(18)$ & $-61(1)$ \\
\hline C(10) & $C(17)$ & $C(16)$ & $H(16)$ & $-176(1)$ & $C(18)$ & $C(17)$ & $C(16)$ & $H(17)$ & $-55(1)$ \\
\hline
\end{tabular}


Table 7. Torsion angles ( 0 ) -- continued

\begin{tabular}{|c|c|c|c|c|c|c|c|c|c|}
\hline$C(18)$ & $C(17)$ & $C(16)$ & $H(18)$ & $64(1)$ & C(15) & $\mathrm{C}(17)$ & $C(16)$ & $H(16)$ & $-61(1)$ \\
\hline$C(15)$ & $C(17)$ & $C(16)$ & $H(17)$ & $58(1)$ & $C(15)$ & $C(17)$ & $C(16)$ & $H(18)$ & $179.0(8)$ \\
\hline$C(11)$ & $C(17)$ & $C(15)$ & $C(14)$ & $68.3(8)$ & $C(11)$ & $C(17)$ & $C(15)$ & $H(14)$ & $-172(1)$ \\
\hline$C(11)$ & $C(17)$ & $C(15)$ & $H(15)$ & $-50(1)$ & $C(18)$ & $C(17)$ & $C(15)$ & $C(14)$ & $-38.1(7)$ \\
\hline$C(18)$ & $C(17)$ & $C(15)$ & $H(14)$ & $81(1)$ & $C(18)$ & $C(17)$ & $C(15)$ & $H(15)$ & $-156(1)$ \\
\hline$C(16)$ & $C(17)$ & $C(15)$ & $C(14)$ & $-163.6(5)$ & $C(16)$ & $C(17)$ & $C(15)$ & $H(14)$ & $-43(1)$ \\
\hline$C(16)$ & $C(17)$ & $C(15)$ & $H(15)$ & $77(1)$ & $C(12)$ & $C(13)$ & $C(14)$ & $C(15)$ & $-75(1)$ \\
\hline$C(12)$ & $C(13)$ & $C(14)$ & $H(12)$ & $43(1)$ & $C(12)$ & $C(13)$ & $C(14)$ & $H(13)$ & $165(1)$ \\
\hline$C(18)$ & $C(13)$ & $C(14)$ & $C(15)$ & $32.4(7)$ & $C(18)$ & $C(13)$ & $C(14)$ & $H(12)$ & $150(1)$ \\
\hline$C(18)$ & $C(13)$ & $C(14)$ & $H(13)$ & $-87(1)$ & $\mathrm{H}(11)$ & $C(13)$ & $C(14)$ & $C(15)$ & $156.8(9)$ \\
\hline$H(11)$ & $C(13)$ & $C(14)$ & $H(12)$ & $-84(1)$ & $H(11)$ & $C(13)$ & $C(14)$ & $H(13)$ & $37(1)$ \\
\hline$C(6)$ & $C(1)$ & $C(2)$ & $C(3)$ & $1(2)$ & $C(6)$ & $C(1)$ & $C(2)$ & $H(2)$ & $179(358)$ \\
\hline$H(1)$ & $C(1)$ & $C(2)$ & C(3) & $-179(718)$ & $H(1)$ & $C(1)$ & $C(2)$ & $H(2)$ & $-2(3)$ \\
\hline$C(26)$ & C(25) & $C(24)$ & C(23) & $1(2)$ & $C(26)$ & $C(25)$ & $C(24)$ & $H(28)$ & $179(803)$ \\
\hline $\mathrm{H}(29)$ & C(25) & C(24) & C(23) & $-177(2)$ & $H(29)$ & $C(25)$ & $C(24)$ & $H(28)$ & $1(3)$ \\
\hline$C(37)$ & $C(35)$ & $C(34)$ & $C(33)$ & $5(1)$ & C(37) & C(35) & C(34) & $H(36)$ & $-112(1)$ \\
\hline$C(37)$ & $C(35)$ & $C(34)$ & $H(37)$ & $125(1)$ & $H(38)$ & $C(35)$ & $C(34)$ & $C(33)$ & $-113(1)$ \\
\hline$H(38)$ & C(35) & C(34) & $H(36)$ & $128(1)$ & $H(38)$ & $\mathrm{C}(35)$ & $\mathrm{C}(34)$ & $H(37)$ & $6(2)$ \\
\hline H(39) & C(35) & C(34) & $C(33)$ & $124(1)$ & $H(39)$ & $C(35)$ & $C(34)$ & $H(36)$ & 6(1) \\
\hline$H(39)$ & $C(35)$ & $C(34)$ & $\mathrm{H}(37)$ & $-115(1)$ & $C(13)$ & $C(14)$ & $C(15)$ & C(17) & $4.4(7)$ \\
\hline$C(13)$ & $C(14)$ & $C(15)$ & $\mathrm{H}(14)$ & $-115(1)$ & $C(13)$ & $C(14)$ & $C(15)$ & $H(15)$ & $122(1)$ \\
\hline$H(12)$ & $C(14)$ & $C(15)$ & $C(17)$ & $-113(1)$ & $H(12)$ & $C(14)$ & $C(15)$ & $H(14)$ & $126(1)$ \\
\hline$H(12)$ & $C(14)$ & $C(15)$ & $H(15)$ & $4(1)$ & $H(13)$ & $C(14)$ & $C(15)$ & C(17) & 124(1) \\
\hline$H(13)$ & $C(14)$ & $C(15)$ & $H(14)$ & $4(1)$ & $H(13)$ & $C(14)$ & $C(15)$ & $H(15)$ & $-117(1)$ \\
\hline$C(6)$ & C(5) & C(4) & C(3) & $0(2)$ & $C(6)$ & C(5) & C(4) & $\mathrm{H}(4)$ & 175(2) \\
\hline$H(5)$ & C(5) & C(4) & $C(3)$ & $176(2)$ & $\mathrm{H}(5)$ & $C(5)$ & $C(4)$ & $H(4)$ & $-8(3)$ \\
\hline$C(26)$ & C(21) & C(22) & C(23) & $1(3)$ & C(26) & $C(21)$ & C(22) & $H(26)$ & $-174(2)$ \\
\hline $\mathrm{H}(25)$ & C(21) & C(22) & $C(23)$ & $-178(2)$ & $H(25)$ & $C(21)$ & $C(22)$ & $H(26)$ & $5(3)$ \\
\hline$C(5)$ & C(4) & $C(3)$ & $C(2)$ & $0(3)$ & C(5) & $\mathrm{C}(4)$ & $C(3)$ & $H(3)$ & $-178(358)$ \\
\hline $\mathrm{H}(4)$ & $C(4)$ & C(3) & $C(2)$ & $-175(2)$ & $\mathrm{H}(4)$ & C(4) & C(3) & $H(3)$ & $6(3)$ \\
\hline$C(24)$ & $C(23)$ & C(22) & $C(21)$ & $0(3)$ & $C(24)$ & $C(23)$ & $C(22)$ & $H(26)$ & $175(2)$ \\
\hline $\mathrm{H}(27)$ & $C(23)$ & $C(22)$ & $C(21)$ & $174(2)$ & $\mathrm{H}(27)$ & $C(23)$ & C(22) & $H(26)$ & $-9(4)$ \\
\hline$C(22)$ & $C(23)$ & $C(24)$ & $C(25)$ & $0(3)$ & C(22) & $C(23)$ & $C(24)$ & $H(28)$ & $-179(507)$ \\
\hline $\mathrm{H}(27)$ & $C(23)$ & $C(24)$ & $C(25)$ & $-175(2)$ & $\mathrm{H}(27)$ & $C(23)$ & $C(24)$ & $H(28)$ & $5(3)$ \\
\hline$C(1)$ & $C(2)$ & $C(3)$ & $C(4)$ & $0(3)$ & $C(1)$ & $\mathrm{C}(2)$ & $C(3)$ & $H(3)$ & $177(2)$ \\
\hline$H(2)$ & $C(2)$ & $C(3)$ & C(4) & $-178(2)$ & $H(2)$ & $C(2)$ & $C(3)$ & $H(3)$ & $0(3)$ \\
\hline
\end{tabular}

The sign is positive if when looking from atom 2 to atom 3 a clock-wise motion of atom 1 would superimpose it on atom 4. 
Table. Summary of Crystallographic Data

\begin{tabular}{|c|c|c|c|c|c|}
\hline & trans-6a & trans-6b & cis-6c & exo-6d & exo-6e \\
\hline formula & $\mathrm{C}_{20} \mathrm{H}_{26} \mathrm{~N}_{2}$ & $\mathrm{C}_{19} \mathrm{H}_{24} \mathrm{~N}_{2}$ & $\mathrm{C}_{17} \mathrm{H}_{20} \mathrm{~N}_{2}$ & $\mathrm{C}_{17} \mathrm{H}_{18} \mathrm{~N}_{2}$ & $\mathrm{C}_{20} \mathrm{H}_{24} \mathrm{~N}_{2}$ \\
\hline mol wt & 294.44 & 280.41 & 252.36 & 250.34 & 292.42 \\
\hline cryst dimens / mm & $0.80 \times 0.30 \times 0.20$ & $0.60 \times 0.20 \times 0.10$ & $0.50 \times 0.25 \times 0.20$ & $0.60 \times 0.50 \times 0.05$ & $0.75 \times 0.50 \times 0.25$ \\
\hline cryst system & monoclinic & monoclinic & orthorhombic & orthorhombic & triclinic \\
\hline space group & $\mathrm{P} 2_{1}$ & $\mathrm{C} 2 / \mathrm{c}$ & $\mathrm{P} 2_{1} 2_{1} 2_{1}$ & $\mathrm{Pna}_{1}$ & $\mathrm{C} 1$ \\
\hline $\mathrm{a} / \AA$ & $16.7104(3)$ & $35.436(3)$ & $10.6730(5)$ & $17.61(2)$ & $11.7618(7)$ \\
\hline b / $\AA$ & $6.4424(1)$ & $6.3489(5)$ & $10.9928(7)$ & $9.93(1)$ & $13.1850(8)$ \\
\hline $\mathrm{c} / \AA$ & $17.8094(4)$ & $15.747(1)$ & $12.8104(9)$ & $8.376(9)$ & $13.7331(8)$ \\
\hline$\alpha / \operatorname{deg}$ & 90 & 90 & 90 & 90 & $92.734(3)$ \\
\hline$\beta / \operatorname{deg}$ & $110.457(2)$ & $97.655(3)$ & 90 & 90 & $112.610(2)$ \\
\hline$\gamma / \operatorname{deg}$ & 90 & 90 & 90 & 90 & $114.056(3)$ \\
\hline$V / \AA^{3}$ & $1796.36(6)$ & $3511.3(5)$ & $1503.0(2)$ & $1463(2)$ & $1741.7(2)$ \\
\hline Z & 4 & 8 & 4 & 4 & 4 \\
\hline $\mathrm{D}_{\text {calcd }} / \mathrm{g} \mathrm{cm}^{-3}$ & 1.09 & 1.061 & 1.115 & 1.136 & 1.115 \\
\hline $\mathrm{F}_{000}$ & 640.00 & 1216.00 & 544.00 & 536.00 & 632.00 \\
\hline$\mu(\mathrm{Mo} \mathrm{Ka}) / \mathrm{cm}^{-1}$ & 0.64 & 0.62 & 0.66 & 0.67 & 0.65 \\
\hline temp / K & 296 & 296 & 296 & 296 & 296 \\
\hline $2 \theta \max / \mathrm{deg}$ & 55.0 & 55.0 & 54.0 & 54.9 & 54.9 \\
\hline no. of reflections & 4342 (Total) & 8316 (Total) & 3792 (Total) & 28490 (Total) & 7730 (Total) \\
\hline measured & 4338 (Unique) & 4158 (Unique) & 1896 (Unique) & 1946 (Unique) & \\
\hline no. observations & $3823(\mathrm{I}>0.50 \mathrm{~s}(\mathrm{I}))$ & $2596(\mathrm{I}>1.00 \mathrm{~s}(\mathrm{I}))$ & 1878 (All reflections) & 1788 (All reflections) & $3717(\mathrm{I}>1.00 \mathrm{~s}(\mathrm{I}))$ \\
\hline no. variables & 449 & 214 & 192 & 190 & 445 \\
\hline rfln/parameter ratio & 8.51 & 12.13 & 9.78 & 9.41 & 8.35 \\
\hline $\mathrm{R}^{a}$ & $0.094(\mathrm{I}>0.50(\mathrm{I}))^{b}$ & $0.102(\mathrm{I}>1.00(\mathrm{I}))$ & 0.111 (All reflections) & 0.142 (All reflections) & $0.063(\mathrm{I}>1.00(\mathrm{I}))$ \\
\hline $\mathrm{R} 1^{a}$ & $0.079(\mathrm{I}>2.00(\mathrm{I}))^{b}$ & $0.091(\mathrm{I}>2.00(\mathrm{I}))$ & $0.061(\mathrm{I}>2.00(\mathrm{I}))$ & $0.098(\mathrm{I}>2.00(\mathrm{I}))$ & $0.061(\mathrm{I}>2.00(\mathrm{I}))$ \\
\hline $\mathrm{Rw} 2^{a}$ & $0.136(\mathrm{I}>0.50(\mathrm{I}))^{b}$ & $0.214(\mathrm{I}>1.00(\mathrm{I}))$ & 0.185 (All reflections) & 0.223 (All reflections) & $0.132(\mathrm{I}>1.00(\mathrm{I}))$ \\
\hline goodness of fit ${ }^{a}$ & $1.030^{\mathrm{b}}$ & 0.885 & 0.908 & 1.228 & 1.059 \\
\hline
\end{tabular}


General Experimental Method. Photoirradiaton was carried out with 300-W high-pressure mercury arc lamp. TLC was performed on silica gel plates; visualization was accomplished by UV light (254 $\mathrm{nm}$ ) or by exposing to $\mathrm{I}_{2}$ vapor. Column chromatography was performed on silica gel. ${ }^{1} \mathrm{H}$ and ${ }^{13} \mathrm{C}$ NMR spectra were obtained with $300 \mathrm{MHz}$ and $75 \mathrm{MHz}$ NMR spectrometers, respectively, using $\mathrm{CDCl}_{3}$ as a solvent and chemical shifts were reported in $\delta$ (parts per million) relative to $\mathrm{Me}_{4} \mathrm{Si}$. Other spectral and analytical data and HPLC separation were performed on standard laboratory instruments.

All commercially available chemicals for syntheses of substrates 1a-e were used without further purification. Acetonitrile was distilled two times over $\mathrm{P}_{2} \mathrm{O}_{5}$ and once over $\mathrm{CaH}_{2}$ before use. Phenanthrene was recrystallized from methanol. Acetic acid and $\mathbf{7}$ were used as purchased. All reactions were carried out under argon atmosphere. 